\title{
X-ray waveguide optics: Beyond straight channels
}

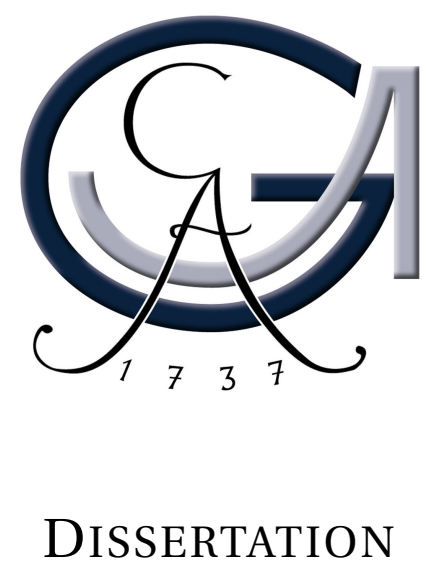

zur Erlangung des mathematisch-naturwissenschaftlichen Doktorgrades

"Doctor rerum naturalium"'

der Georg-August-Universität Göttingen

im Promotionsprogramm ProPhys

der Georg-August University School of Science (GAUSS)

vorgelegt von

SARAH HofFMANN-URLAUB

aus Celle

Göttingen 2016 


\section{Mitglieder des Betreuungsausschusses:}

Referent:

1. Korreferent:
Prof. Dr. Tim Salditt

Institut fr Röntgenphysik

Prof. Dr. Ulrich Krebs

Institut fr Materialphysik

\section{Weitere Mitglieder der Prüfungskommission:}

Dr. Alexander Egner, Laser Laboratorium

Prof. Dr. Sarah Köster, Institut fr Röntgenphysik

Prof. Dr. Angela Rizzi, VI. Physikalisches Institut

Prof. Dr. Andreas Tilgner, VI. Physikalisches Institut

\section{Tag der mündlichen Prüfung:}

18. Oktober 2016 


\section{Contents}

1 Introduction 1

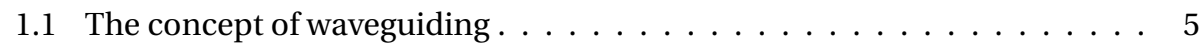

1.2 Propagation in advanced geometries $\ldots \ldots \ldots \ldots \ldots \ldots$

1.2 .1 Single straight channels $\ldots \ldots \ldots \ldots \ldots \ldots$

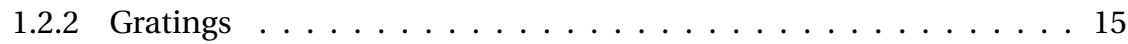

1.2 .3 Tapered channels . . . . . . . . . . . . . . . . . . . . . 20

1.2 .4 Curved channels . . . . . . . . . . . . . . . . . . . . . . . . . 24

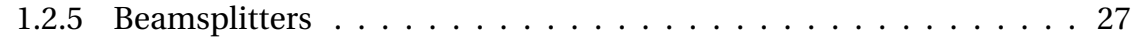

1.3 Probe reconstruction . . . . . . . . . . . . . . . . . . . . . . 29

1.4 Characterization of $\mathrm{x}$-ray beams $\ldots \ldots \ldots \ldots \ldots \ldots$

2 High aspect ratio $x$-ray wave-guide channels fabricated by e-beam lithography and wafer bonding 39

\begin{tabular}{|lll}
\hline 3 & Advances in x-ray waveguide fabrication & 47
\end{tabular}

\begin{tabular}{|ll|}
4 & X-ray beam compression by tapered waveguides \\
\hline
\end{tabular}

5 Supplemental Material 59

6 Miniaturized beamsplitters realized by x-ray waveguides 69

\begin{tabular}{lll}
\hline 7 & Summary and concluding remarks & 79
\end{tabular}

\begin{tabular}{|lc|}
\hline$A$ The waveguide universe & 83
\end{tabular}

\begin{tabular}{|ll}
\hline B PyPropagate source code & 87
\end{tabular}

$\begin{array}{ll}\text { Bibliography } & 111\end{array}$

\begin{tabular}{ll}
\hline Authors contribution & 119
\end{tabular}

\begin{tabular}{ll}
\hline Acknowledgements & 121
\end{tabular} 



\section{Chapter 1}

\section{Introduction}

In order to achieve a better understanding of functional processes in nature, researchers have always aimed at gaining deeper insights into matter, particularly on microscopic length scales [1. As a major probe, visible light has since been used for optical imaging. However, this often limits the information depth to the surface or surface-near regions of the specimen, due to the low penetration depth of visible light for most materials. Moreover, according to Abbe's law [2] the resolution of the visualized structure inside transparent objects is limited by the wavelength used for inspection, at least in conventional microscopes.

Because short wavelengths are able to resolve fine features and furthermore provide a higher penetration depth, the discovery of x-rays by W.C. Röntgen [3] was a cornerstone for research. As x-rays cover the wavelength range from $0.01-10 \mathrm{~nm}$, information about the inner texture of comparatively thick and optically opaque media can be accessed at high resolution. Using the Beer-Lambert law [4], the penetration depth can be calculated for a predefined transmission, when the attenuation coefficient for the specific radiation energy/wavelength is known. This coefficient comprises of several interaction mechanisms of electromagnetic waves and matter, but is dominated by absorption of photons according to the photoeffect.

Apart from absorption, specimens can also impart a phase shift on the transmitted electromagnetic waves, meaning that the oscillation states (for given time and space) of two waves are shifted with respect to each other. Distinct from the absorption, this second type of contrast mechanism is used by a variety of imaging techniques such as phase contrast microscopy [5, 6], phase contrast tomography [7, 8] and holography [9] in in-line [10, 11, 12] and off-axis geometry [13]. To fully exploit this contrast, waves are required which exhibit a fixed phase correlation in view of the spatial and temporal propagation, denoted as coherence. Since only intensities (modulus square of the wave amplitude) can be measured by x-ray detectors? phases or relative phase-shifts, respectively, have to be made visible by interference and retrieved by iterative algorithms [14, 15].

1 Through the photoelectric effect, free electrons are generated by the x-rays in a semiconductor material leading to the buildup of a measurable photocurrent. 
To advance these techniques, there is a demand for specialized x-ray sources and optics designed for assessing structures on fundamental length scales, in particular on molecular scales in the micron and nano range. For high resolution imaging the sources must be both intense (at least $10^{10}$ photons per second) and (sub-) micron-sized as well. Fabricating suitable optical elements is by no means a trivial task because the concept of focusing is based on the interaction of radiation and matter, which is comparably weak for x-rays. For this reason, conventional optics such as lenses from a single material are inappropriate to perform this task. In the past few decades significant progress was achieved for a multitude of x-ray optical elements such as compound refractive lenses (CRL), multilayer Laue lenses (MLL), Fresnel zone plates (FZP), multilayer zone plates (MZP) and waveguides (WG), concerning the available flux behind the device and the focal spot size. In principle, these optics enable a non-destructive and particularly non-invasive examination of processes in high-resolution. They can be classified into three key concepts of operation:

Reflection: multilayer mirrors [16, 17], bent or asymmetrically cut crystals [18], capillaries [19]

Refraction: CRL 20, 21]

Diffraction: gratings [22], FZP [23], MZP [24], MLL [25] 26] and waveguides [27, 28

Here, the weak interaction with matter poses major challenges for the fabrication of the optics listed above. Especially in view of obtaining intense and simultaneously small foci it is crucial to ensure an accuracy in production on the nano scale over lateral dimensions ranging up to several millimeters. Benchmarks of the current state of research for different types of $\mathrm{x}$-ray optics are compiled in table 1.1

\begin{tabular}{|c|c|c|c|c|c|}
\hline Type & Spot size $[\mathrm{nm}]$ & $\mathrm{E}_{\mathrm{ph}}[\mathrm{keV}]$ & Transmission [\%] & Materials & Published by \\
\hline$\overline{\mathrm{CRL}}$ & $50 \times 50$ & 21 & 30.5 & $\mathrm{Si}$ & $\overline{2005, \text { Schroer } \text { et al. } 29}$ \\
\hline MLL & $25 \times 27$ & 12 & 2 & $\mathrm{Si} / \mathrm{WSi}_{2}$ & 2011, Yan et al. 30 \\
\hline FZP & $58 \times 58$ & 8 & 5 & $\mathrm{Ta}$ & 2005, Suzuki et al. 31 \\
\hline MZP & $<5 \times<5$ & 7.9 & 2 & $\mathrm{Si} / \mathrm{W}$ & 2013, Dring et al. 32 \\
\hline \multirow[t]{2}{*}{ WG } & $47 \times 25$ & 12.5 & 4.7 & PMMA & 2005, Jarre et al. 33 \\
\hline & $10 \times 10$ & 13.5 & 14 & $\mathrm{Ge} / \mathrm{Mo} / \mathrm{C} / \mathrm{Mo} / \mathrm{Ge}$ & 2012, Krger et al. 34 \\
\hline
\end{tabular}

Table 1.1: State of research for common $x$-ray optical devices. For different types of $x$-ray optics the twodimensional focal sizes are listed, in particular, comparing the operation energies, transmission values and material compositions. The transmission is calculated by comparing the intensity of the incoming $x$-ray beam (fully illuminating the optical element) to the signal measured behind the device.

Picked from this multitude of x-ray optics, waveguides are addressed in this thesis. Their functionality basically resembles an inverted fiber optic. A guiding material with low density is imbedded in a high density cladding. In the guiding part the wave field propagates in the form of resonant oscillations (modes). 
In view of the functioning and structure of waveguides the following approaches for one-dimensional (1D) and two-dimensional (2D) focusing are pursued:

In the case of resonant beam couplers [28, 35, 36, 37] the radiation is coupled into the guiding layer (potentially a multilayer [38, 39, 40]) through a thin top-layer and is focused in one direction. Contrarily, in front coupling geometry the radiation impinges on the entrance side of one-dimensionally [41] or two-dimensionally confined channels, filled with polymer [42] or air [43].

The 2D focusing waveguides specified in table 1.1 both consist of a weakly absorbing core (carbon or polymer) in a non-transparent semi-conductor cladding. A solid core is disadvantageous in view of a reduced transmission and radiation damage [4, 45, affecting the guiding properties. These limitations can be overcome by fabricating hollow channels.

Proof of principal experiments using lithographically fabricated, air-filled channel waveguides imbedded in silicon were published by Kohlstedt et al. [43], where micron-sized channels revealed transmissions of $<1 \%$. Significant progress was then achieved by Neubauer [46], in particular in terms of down-sizing the channel dimensions, aiming at coherent exit fields by mode-filtering.

Continuing this pioneering work, one goal of this thesis is the enhancement of the optical efficiency to a level sufficient for imaging applications. Waveguides are especially suitable for holographic x-ray imaging experiments, since the divergent beam leaving the channel entails a geometric magnification, and exhibits a high degree of coherence [47, 48 as well as a clean spherical wave-front. They can be considered as virtual point-sources due to the small dimensions of the channels (cross-sections of several tens of nanometers [49]) and the inherent size of the modes [50]. To provide a high quality intensity distribution behind the waveguide (near-field), all fabrication steps such as spin-coating, electron beam lithography, wet development, reactive ion etching and wafer bonding are optimized and coordinated with respect to each other at the same time, since every single step depends on the previous procedure. For each production step the crucial parameters are identified and the corresponding values are determined precisely. Build on the basis of high accuracy and reliability in manufacturing, waveguide channels can be fabricated in advanced geometries such as gratings, tapered and curved channels, or beamsplitters, enabling for constructing novel x-ray tool as for example time delay devices or interferometers [51, 52].

Furthermore, the propagation of the electromagnetic field inside the waveguide channel can be numerically studied, using finite difference simulations [53]. This method not only yields a tool for gaining access to a deeper understanding of the waveguiding mechanism, but also allows to optimize the channel geometry in order to enhance the efficiency of the guide and to define the intensity distribution in the near-field regime. Along with meeting the requirements of both the measurement setup and the sample, the propagation of the wave field can thus be predicted beforehand and compared to the findings from phase retrieval algorithms afterwards. Hence, waveguide fabrication can be considered as a key technology for x-ray beam tailoring. 


\section{Outline}

This thesis develops advanced $\mathrm{x}$-ray waveguide optics based on lithographically fabricated channels. Starting from the theoretical framework, different waveguide designs are considered and specific benefits and limitations are discussed. Combining finite difference simulations and experimental results, waveguides in various geometries are characterized extensively.

A compilation of four publications is included with the latest improvements in waveguide fabrication and their optical performance. Chapter 1 provides a broad introduction into the theoretical background of waveguiding in different channel geometries and arrangements, presenting a framework for the experimental results articulated in chapters 4 and 6. Also the typical geometric dimensions of guiding channels are considered, leading to an understanding of the requirements for fabrication methods, detailed in chapters 2 and 3 . Furthermore, reconstructions of the near-field of single channels and gratings as well as waveguides utilized for beam characterization are presented to complete the view on x-ray waveguides.

In chapter 2, the process of fabricating empty channels in a silicon cladding is presented along with results of x-ray holography, reconstruction algorithms and simulations of wave fields inside the waveguides.

Proceedings of the manufacturing process of nano-sized channels are shown in chapter 3 The process parameters are optimized, accomplishing new benchmarks in $\mathrm{x}$-ray optical performance for waveguide channels in silicon. Finally, the fabrication procedure is extended to other materials such as germanium and quartz.

The optical functionality of tapered waveguides and beamsplitters is demonstrated in chapters 4 (with attached supplemental material) and 6. The experimental results confirm the analytical predictions and additionally reveal interesting features in the wave field not only within the channel but also in the near- and far-field regime.

In chapter 7 the findings of this thesis are summarized and an outlook concerning future investigations in this field is given. 


\subsection{The concept of waveguiding}

In this section the theoretical background of hard $x$-ray waveguiding is presented in excerpts, while a detailed monograph can be found in [54, 55]. Regarding the propagation of wave fields in guiding structures, some fundamentals of $x$-ray optics are included which are necessary to understand the general concept and to evaluate the results from experiments and simulations.

In this part the term waveguide is explicitly used for an air-/vacuum-filled channel (core) imbedded in a silicon cladding, whereas other materials for the cladding such as germanium or quartz are considered in chapter 3 . The optical properties of a material are quantified by the index of refraction $n=c_{\text {vacuum }} / c_{\text {medium }}$, describing the ratio of speed of light $c$ in vacuum and medium. When radiation passes through matter, absorption (reduced transmission) and dispersion (wavelength dependency of the propagation velocity) are observed which are expressed by the corresponding coefficients $\beta$ and $\delta$, such that

$$
n=1-\delta+\mathrm{i} \beta .
$$

For x-rays at a wavelength $\lambda=0.01-10 \mathrm{~nm}$, the coefficients are: $\delta \backsim 10^{-5}-10^{-9}$ and $\beta \approx \delta \cdot 10^{-2}$, with exact values depending on the material composition.
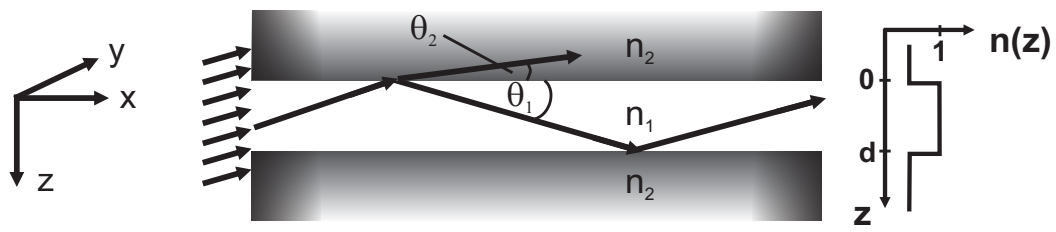

Figure 1.1: Principle of waveguiding. An incoming wave field is coupled into a channel from the left (frontend), impinges several times on the interface between the two media with the refractive indices $n_{1}$ and $n_{2}$, before exiting the channel on the right side. Assuming an air/vacuum-filled core and a silicon cladding, $n_{1}$ is larger than $n_{2}$.

In figure 1.1 a single ray trajectory of the incident wave field is traced for illustration. The refraction at the boundaries can be described by Snell's law

$$
n_{1} \cos \theta_{1}=n_{2} \cos \theta_{2} \quad \text { for } \quad n_{1}>n_{2} \text {. }
$$

There is a critical angle $\cos \theta_{c}=n_{2} / n_{1}$, indicating a material-dependent threshold. Above this value the rays enter the cladding $\left(\theta>\theta_{c}\right)$; below this angle the radiation is reflected at the surface $\left(\theta<\theta_{c}\right)$. Since $\delta$ depends on the photon energy $E_{p h}$, the same applies for the angle $\theta_{c}=\sqrt{2 \delta}$. For silicon at $E_{p h}=7.9 \mathrm{keV}$, the critical angle for total reflection is $\theta_{c}=0.227^{\circ}$ [56].

A guided wave traveling in positive $x$-direction can be described by:

$$
\Psi=\Psi(z) e^{i\left(\omega t-\beta_{d} x\right)}
$$

where $\omega=k \cdot c / n$ is the angular frequency of a wave field (harmonic in time $t$ ) with wave number $k=2 \pi / \lambda$, and $\beta_{d}=n_{1} k \cos \theta_{1}$ as the damping coefficient. 
Equation [1.2] is a solution of the wave equation

$$
\left(\nabla^{2}-\frac{n^{2}}{c^{2}} \partial_{t}^{2}\right) \Psi=0 \quad \text { with } \quad \nabla=\mathbf{e}_{x} \partial / \partial x+\mathbf{e}_{y} \partial / \partial y+\mathbf{e}_{z} \partial / \partial z
$$

and $\mathbf{e}_{f}$ as the unit vector in $f$-direction. Inserting Eq. 1.2 into Eq. 1.3 leads to the reduced Helmholtz equation

$$
\frac{\partial^{2}}{\partial z^{2}} \Psi(z)+\left(n^{2} k^{2}-\beta_{d}^{2}\right) \Psi(z)=0 .
$$

The continuity at the interfaces at $z=0$ and $z=d$ and a vanishing wave for $z= \pm \infty$ require solutions for the amplitude $\Psi(z)$ that fulfill the following constraints:

$$
\Psi(z)=\left\{\begin{array}{llr}
A e^{\gamma z} & \text { for } & z \leq 0 \\
B \cos \kappa z+C \sin \kappa z & \text { for } & d \geq z \geq 0 \\
D e^{-\gamma(z-d)} & \text { for } & z \geq d .
\end{array}\right.
$$

Here, the field inside the channel ( $d \geq z \geq 0)$ is a superposition of two plane waves (sine and cosine terms for odd and even modes, respectively). The parameters

$$
\begin{aligned}
\kappa & =\sqrt{n_{1}^{2} k^{2}-\beta_{d}^{2}}, \\
\gamma & =\sqrt{\beta_{d}^{2}-n_{2}^{2} k^{2}}
\end{aligned}
$$

only result in physically plausible solutions inside the channel if $\kappa, \gamma \in \mathbb{R}$, otherwise the field would be extended towards infinity without any attenuation; this is not fulfilled when $n_{2} k \geq \beta_{d}=n_{1} k \cos \theta_{1}=n_{2} k$ for $\theta=\theta_{c}$. As mentioned above, the critical angle $\theta_{c}$ assigns a threshold where the impinging field is no longer guided within the channel, but is turned into a radiating field that is successively absorbed in the cladding material.

A homogeneous system of equations has to be solved to maintain the parameters in Eq. [1.5. This leads to the eigenvalue equation

$$
\tan (\kappa d)=\frac{2 \kappa \gamma}{\kappa^{2}-\gamma^{2}}=\frac{2 \kappa d \sqrt{\left(n_{1}^{2}-n_{2}^{2}\right) \cdot(k d)^{2}-(\kappa d)^{2}}}{(\kappa d)^{2}-\left[\left(n_{1}^{2}-n_{2}^{2}\right) \cdot(k d)^{2}-(\kappa d)^{2}\right]}=: F(\kappa d) .
$$

The numerator of the function $F(\kappa d)$ vanishes when

$$
\kappa d \geq \sqrt{n_{1}^{2}-n_{2}^{2}} \cdot k d=: V
$$

is reached. For even larger $\kappa d$, the root in Eq. 1.6 becomes imaginary and the function $F(\kappa d)$ ends at point $V$, which is hence called cutoff. From equation (1.6) the number of the modes $M$ in a channel with a certain width $d$ can estimated graphically (see Fig. 1.2 . 

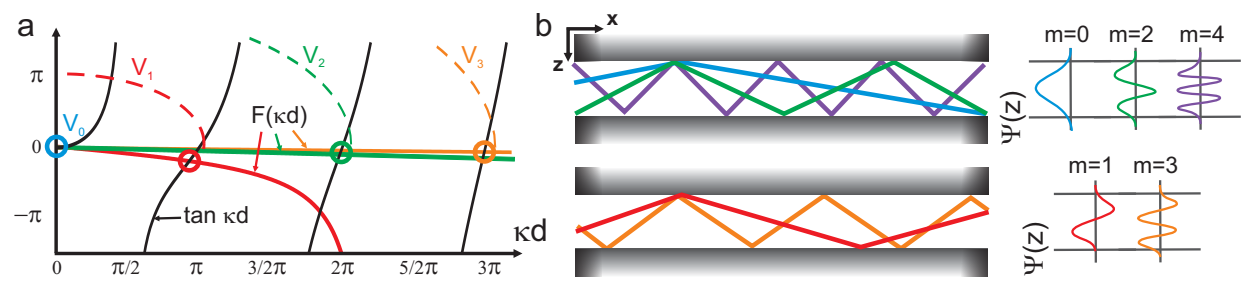

Figure 1.2: Graphical solution of the eigenvalue problem. (a) For varying $\kappa d$ the tangent function, $F(\kappa d)$ and the cutoff conditions $V_{m}=\left[V^{2}-(\kappa d)^{2}\right]^{1 / 2}$ for the first four modes are plotted. All crossings of the two functions (marked with colored circles) inside a certain circular orbit $V_{m}$ represent solutions of the eigenvalue equation [1.6]. (b) The propagation of the first five modes inside a waveguide is drawn schematically. On the right hand side the amplitude $\Psi(z)$ of the wave field is plotted, indicating that the electromagnetic wave can be analyzed analogously to an electron, trapped in a potential well. For even modes $(m=0,2,4)$, the amplitude is symmetrical to the optical axis in $x$-direction, whereas odd modes $(m=1,3)$ reveal a point symmetry.

The crossing points of the black and solid colored lines represent solutions of the eigenvalue equation (1.6). The number of $\pi$-crossings of $F(\kappa d)$ before reaching the cutoff value $V_{m}$ (with $m=0,1,2, \ldots$ ) which is depicted as colored dashed lines, is equal to the number of guided modes

$$
M=\left\lceil\frac{V}{\pi}\right\rceil \quad \text { with }\lceil b\rceil:=\min [a \in \mathbb{Z} \mid a \geq b] .
$$

For increasing channel widths, the cutoff is shifted to the right, resulting in a higher number of intersections with the tangent function and a correspondingly higher number of modes [see Fig. 1.2(a)]. Since $F(\kappa d)$ and the tangent function share the same origin, there is always one (fundamental) mode guided in a narrow channel $\left(M\left(V_{m=0}\right)=1\right)$. Note that for illumination parallel to the optical axis, only mirrorsymmetric (even) modes are excited.

Neglecting absorption $(\beta=0)$ for an air/vacuum-filled channel, the refractive index:

$$
n_{S i}=1-\delta=1-\frac{\lambda^{2}}{2 \pi} r_{0} \rho_{S i} \quad \text { with } \quad \begin{aligned}
r_{0} & =2.82 \cdot 10^{-15} \mathrm{~m} \quad \text { (classical el. }- \text { radius) } \\
\rho_{S i} & =0.0248 \mathrm{~nm}^{-2} \quad \text { (el. }- \text { density) }
\end{aligned}
$$

can be used to estimate the critical width $d_{c}$ for guiding a second mode from Eq. [1.7):

$$
V=\sqrt{4 \pi r_{0} \rho_{S i}} d \stackrel{V_{m=0}=\pi}{\longrightarrow} d_{c}=\frac{1}{2} \sqrt{\frac{\pi}{r_{0} \rho_{S i}}} .
$$

For silicon the critical width is $d_{c} \approx 20 \mathrm{~nm}$, in wider channels the propagation of multimodal wave fields is enabled. 


\subsection{Propagation in advanced geometries}

In this section the $x$-ray optical properties of waveguides in different geometries are presented combining analytical studies, finite difference simulations (FDSs) and experimental results.

In all simulations, gray lines and filled areas mark borders of the solid silicon cladding. Furthermore, schematic drawings illustrate the parameters suited to describe each type of channel geometry. As a function of those parameters, the ratio of the intensities entering $\left(I_{i n}\right)$ and leaving the channel $\left(I_{\text {out }}\right)$ is plotted. In this way the optical performance of the channel is quantified while varying its dimensions. All intensities are normalized to the input plane wave. The simulated data points for $I_{\text {out }} / I_{\text {in }}$ as well as the measured values for the transmission $T$ are connected by lines, serving solely as a guide to the eye. In the simulations, sharp boundaries are assumed, without accounting for any defective structures. Since most of the displayed measurements were performed at $E_{p h}=7.9 \mathrm{keV}$ (except for the gratings), this energy was also chosen for the FDSs. All lengths $L$, spacings $S$, widths $d$ and depths $h$ of the channels are measured using a scanning electron microscope (SEM), revealing a precision of $\pm 4 \mathrm{~nm}$ for a magnification factor of $50 \cdot 10^{3}$.

\subsubsection{Single straight channels}

In the previous section, the basic concept of a single straight waveguide channel was introduced. For this well known model system, one-dimensionally (1D) and two-dimensionally (2D) simulations are performed using a finite difference solver [53].

In the simulations ${ }^{2}$ a waveguide with variable width $d$ and length $L=1 \mathrm{~mm}$ is imbedded in a box of $4 \mu \mathrm{m} \times 1.3 \mathrm{~mm}(\mathrm{x}, \mathrm{z})$, sampled with $4000 \times 1300$ pixels. For the $2 \mathrm{D}$ case, a third simulation box of 2000 pixels is chosen, covering $2 \mu \mathrm{m}$ in $y$-direction. Additionally to the wave field propagating in the channel, free space propagation is simulated $20 \mu \mathrm{m}$ in front of and $280 \mu \mathrm{m}$ behind the waveguide structure.

2 Templates for modeling waveguides in various geometries are given in Appendix B. 


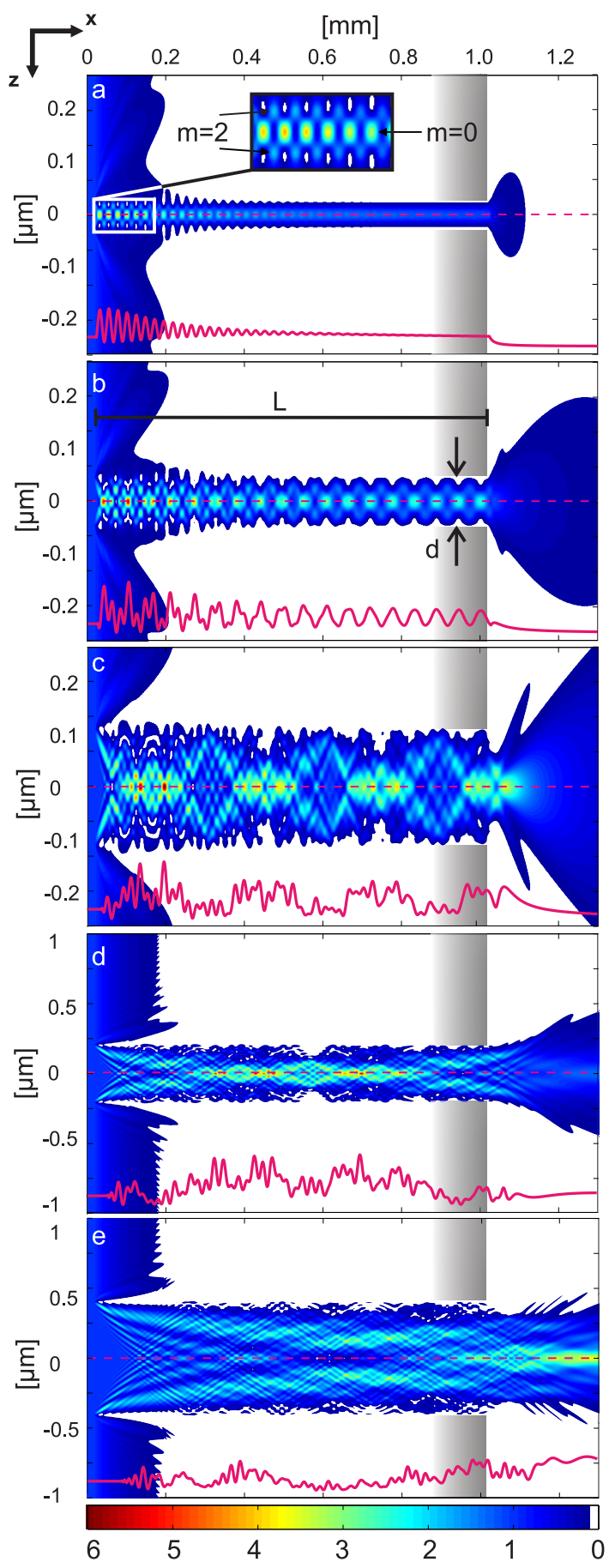



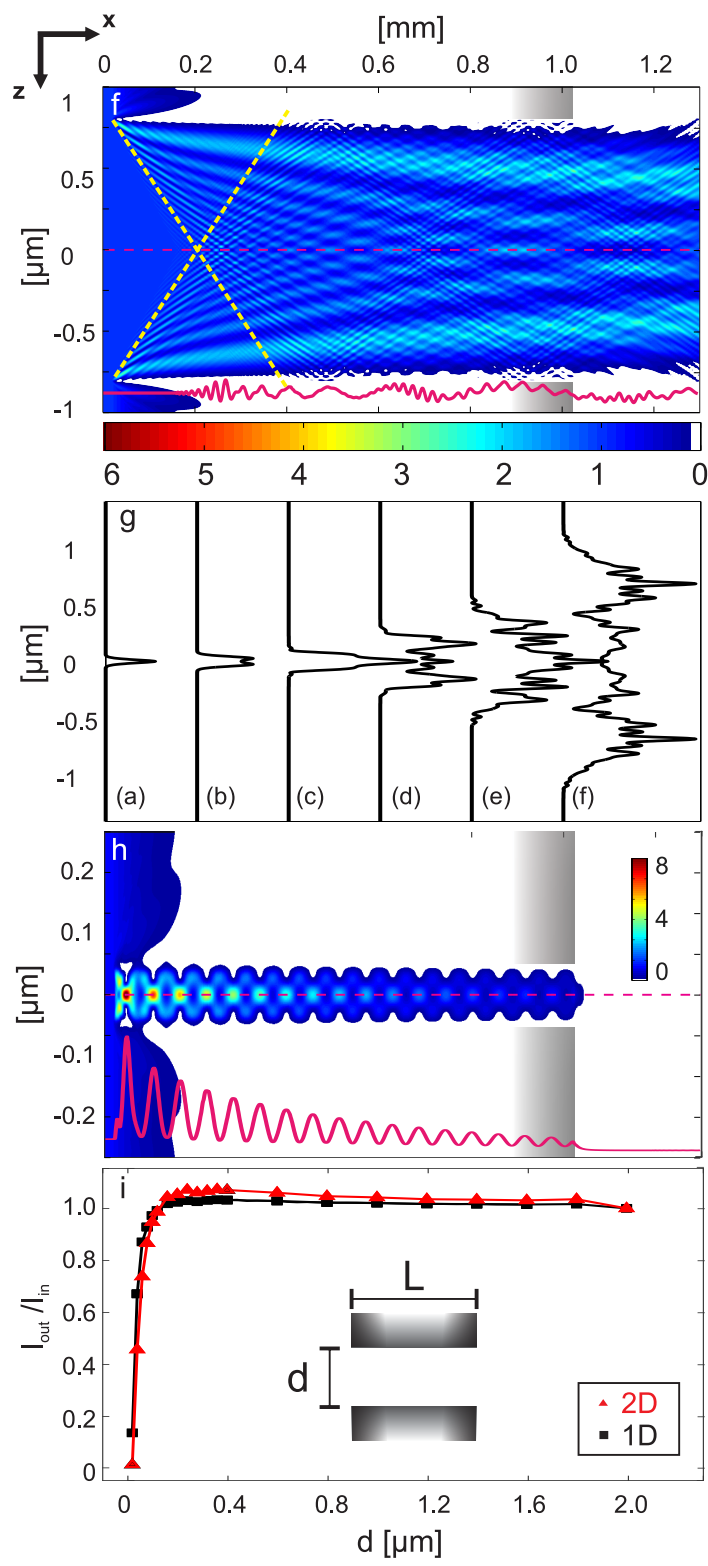

Figure 1.3: Guiding properties of straight channels. FDSs of straight waveguides with different widths $d$ of (a) $40 \mathrm{~nm},(b) 80 \mathrm{~nm},(c) 200 \mathrm{~nm},(d) 400 \mathrm{~nm},(e) 800 \mathrm{~nm}$ and (f) $1600 \mathrm{~nm}$. An intensity profile, extracted at the dashed magenta line $(y=z=0)$, is drawn in the same color at the bottom of each simulated field. (g) Intensity profiles through the exit planes for all channel widths. (h) Slice through the $y=0$ plane of a two-dimensional simulation for a channel with an entrance area of $80 \mathrm{~nm} \times 80 \mathrm{~nm}$. (i) The $I_{\text {out }} / I_{\text {in }}$ ratio as a function of the channel width $d$ for $1 D$ (black squares) and $2 D$ (red triangles) channels. All intensities are normalized to the input intensity. 
In figure 1.3, one-dimensional channels with different widths $d$ are detailed in (a-f). In (a), the inset shows the entrance region in a magnified view. In this channel of width $d=40 \mathrm{~nm}$ two modes are guided, the fundamental mode $(m=0)$ and the first (even) excited mode $(m=2)$. While the intensity of the fundamental mode is located along the center of the channel at $z=0$, the two maxima in intensity of the $m=2$ mode are located close to the borders at $z= \pm d / 2$, as shown above [compare Fig. 1.2(b)]. Thus the profile along $z=0$ is dominated by the $m=0$ mode, revealing a sinusoidal oscillation that decays towards the end of the channel. The effect of modes being damped out is more obvious in figure 1.3 (b). Here, the number of modes, propagating near the entrance side, is reduced in $x$ - and $z$-direction. Especially the higher modes are damped out faster, since the damping coefficient $\beta_{d}$ increases with the mode number $m$ [57]:

$$
\begin{aligned}
\beta_{d} & \approx \frac{\pi^{2}}{d^{3}}(m+1) \frac{\operatorname{Im}\left(\epsilon_{S i} / \epsilon_{V a c}\right)}{\sqrt{\operatorname{Re}\left(1-\epsilon_{S i} / \epsilon_{V a c}\right)}} \frac{\lambda^{2}}{(2 \pi)^{2}} \approx \frac{\lambda^{2}}{4 d^{3}}(m+1) \frac{\operatorname{Im}\left(\epsilon_{S i}\right)}{\sqrt{\operatorname{Re}\left(1-\epsilon_{S i}\right)}}{ }^{3} \\
& =\frac{\lambda^{2}}{4 d^{3}}(m+1) \frac{\operatorname{Im}(1-\delta+i \beta)^{2}}{\sqrt{\operatorname{Re}\left(1-(1-\delta+i \beta)^{2}\right)^{3}}}=\frac{\lambda^{2}}{4 d^{3}}(m+1) \frac{\beta^{2}}{\sqrt{2 \delta}^{3}}=\frac{\lambda^{2}}{4 d^{3}}(m+1) \frac{\beta^{2}}{\theta_{c}^{3}} .
\end{aligned}
$$

The relations $n_{r}=\sqrt{\epsilon_{r} \mu_{r}} \approx \sqrt{\epsilon_{r}}$ for the dielectric permittivity $\epsilon_{r}$ (while the magnetic permeability $\mu_{r} \approx 1$ and $n_{v a c}=1$ ) and $\theta_{c}=\sqrt{2 \delta}$ were used for simplification, while neglecting the $\delta^{2}$-term. In particular, the damping of higher modes is observed in the profile along the channel, where the multi-frequency oscillation is filtered to a sinusoidal shape. As another consequence, the degree of complexity in the mode structure increases with the channel width $d$, evidenced by intensity profiles through the exit planes [see Fig. 1.3 (g)]. In general, this waveguiding effect, namely the occurrence of interference patterns inside (x-ray) optics, has to be taken into account, as long as a wave reflected at one channel border reaches the opposite boundary within the waveguide length $L$. This means that two waves, reflected at opposite channel borders in the entrance side, intersect within the channel [dashed yellow lines in (f)], which is quantified by the width

$$
d_{a}=L \cdot \tan \theta_{c} \approx 4 \mu \mathrm{m} \text { for } L=1000 \mu \mathrm{m} \text { and } \theta_{c}=0.227^{\circ} .
$$

In channels exceeding this threshold, the impact of the interfaces is limited to the area near the channel wall [58]. For channels with $d_{a}>d>d_{c}$, an incoherent illumination results in a partially coherent exit field, where the degree of coherence increases towards unity as the number of modes decreases to the mono-modal regime. For the farfields analyzed in this thesis, a coherent superposition of resonant modes is assumed, since the typical value of $d=100 \mathrm{~nm}$ is close to $d_{c}=20 \mathrm{~nm}$ incoherence effects do not have to be concerned. The $I_{\text {out }} / I_{\text {in }}$ ratio of the waveguides is depicted in figure 1.3 (i). For very small channels, the transmission is low because the amount of the mode propagating in the cladding is higher for narrow channels 45]. Square shaped 2D channels (red triangles) are even affected twice by the same effect due to the confinement in the second ( $y$-)direction. As a result, the intensities for small channel widths $d$ are systematically lower compared to $1 \mathrm{D}$ channels. However, the maximum intensity inside the channel is much larger in $2 \mathrm{D}$ channels, since modes confined in two directions 
are superposed (h). In both cases $I_{\text {out }} / I_{\text {in }}>1$ for broader channels, because radiation impinging onto the front-end propagates inside the channel (assuming $\theta<\theta_{c}$ ); furthermore, the evanescent field "leaks back" into the channel. The percentage of this gained intensity, referring to the total value of incoupled intensity decreases with increasing width $d$; therefore the $I_{\text {out }} / I_{\text {in }}$ ratio declines towards unity.

So far, only the wave field inside a waveguide has been considered. FDSs of the nearfield behind a waveguide channel with a cross-sectional area of $100 \mathrm{~nm} \times 100 \mathrm{~nm}$ are depicted in figure 1.4 . For the simulations in (a-c) a sampling of $8 \mathrm{~nm} / \mathrm{px}$ was chosen in $y$ - and $z$-direction and $10 \mu \mathrm{m} / \mathrm{px}$ in $x$-direction (d).

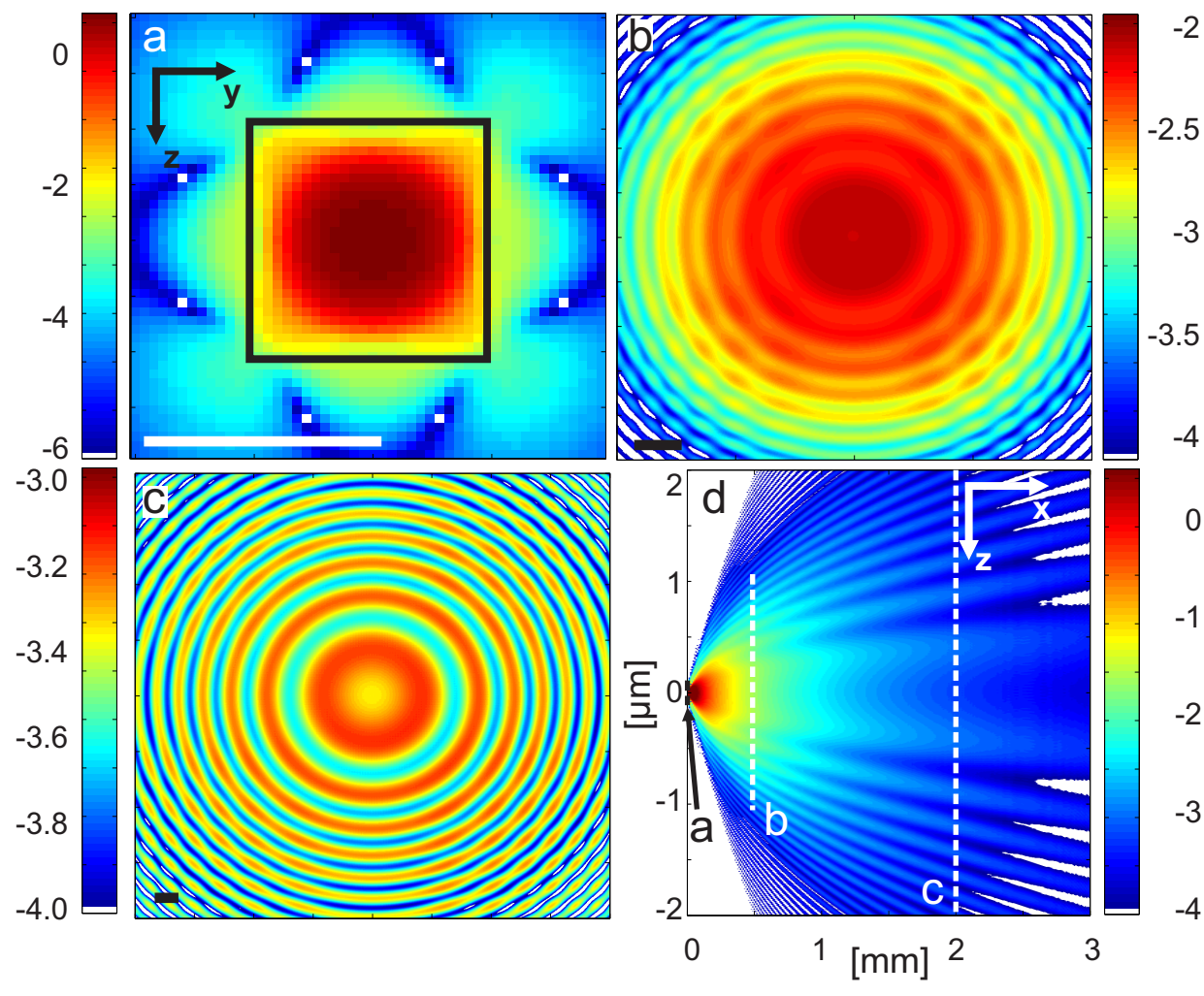

Figure 1.4: Simulations of the exit field intensity. Front view (yz-plane) of the near-field of a $100 \times 100 \mathrm{~nm}^{2}$ sized channel at different $x$-positions of (a) $0 \mu \mathrm{m}$, (b) $500 \mu \mathrm{m}$ and (c) $2000 \mu \mathrm{m}$. Scale bars denote $0.1 \mu \mathrm{m}$ (a) and $0.2 \mu \mathrm{m}(b, c)$, respectively. (d) Section through the wave field at $y=0$. All intensities are normalized to the input intensity and plotted in logarithmic scaling.

The intensity distribution in the exit plane of the waveguide (a) reveals a roundish peak located in the channel center. Outside the channel borders (marked by a black line) the intensity decays rapidly. The radial symmetric peak broadens during propagation (b) resulting in a pattern of concentric, ring-like maxima (c). In (d), a slice of the wave field through the $y=0$ plane clearly illustrates the divergence of the radiation of a few mrad which is typical for waveguide channels. The far-field pattern is the Fourier transfor- 
mation of the intensity distribution in the exit plane and reveals a central maximum of circular shape which is similar to (b). As a benefit for imaging applications, this cone beam illumination entails an inherent magnification of an irradiated sample at the detector position. Next, experimental results for straight channels are presented.

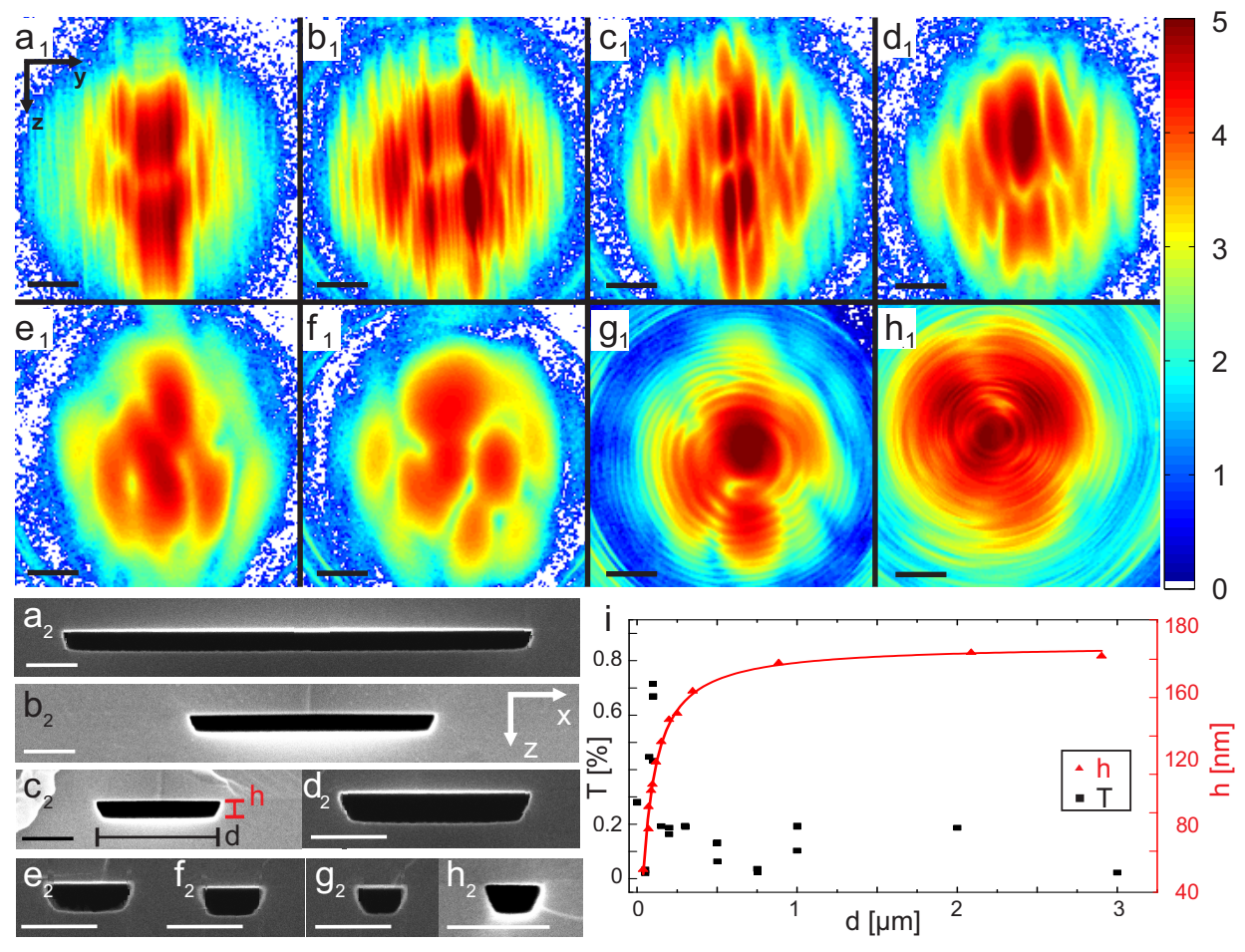

Figure 1.5: Experimental results for straight waveguides. Far-field patterns for different channel sizes of (a) $2750 \mathrm{~nm} \times 81 \mathrm{~nm}$, (b) $983 \mathrm{~nm} \times 82 \mathrm{~nm}$, (c) $491 \mathrm{~nm} \times 83 \mathrm{~nm}$, (d) $317 \mathrm{~nm} \times 79 \mathrm{~nm}$, (e) $211 \mathrm{~nm} \times 79 \mathrm{~nm},(f) 153 \mathrm{~nm} \times 73 \mathrm{~nm}$, (g) $113 \mathrm{~nm} \times 70 \mathrm{~nm}$ and $(\mathrm{h}) 82 \mathrm{~nm} \times 67 \mathrm{~nm}$. The intensity is given in counts per second and plotted in logarithmic scaling. Scale bars denote $0.5 \mathrm{~mm}$. The associated SEM micrographs are labeled by an index 2. Scale bars denote $0.2 \mu \mathrm{m}$. (i) Transmission $T$ and depth $h$ both as a function of width $d$.

Far-field patterns of waveguides with various exit sizes are depicted in figure $1.5\left(\mathrm{a}_{1}-\mathrm{h}_{1}\right)$, the corresponding SEM micrographs are labeled with $\left(\mathrm{a}_{2}-\mathrm{h}_{2}\right)$. The data was recorded $5.1 \mathrm{~m}$ behind the waveguide device by a Pilatus 300k (Dectris) detector at beamline P10 [Deutsches Elektronen Synchrotron (DESY, Hamburg)], at $E_{p h}=7.9 \mathrm{keV}$. The frequency of the oscillations in the $z$-direction is lower than in the $y$-direction, since the channels are stronger confined vertically. This multitude of modes is reduced with decreasing channel size, resulting in an approximately square shaped channel with an equated number of oscillations in both directions (h). In (i), the transmission $T$ (black squares) of the channels is calculated following to the procedure introduced in chapter 4. The transmission decreases for very large and very small channels and exhibits a maximum in between. The high statistical spread of the data is due to under- 
performing channels which are blocked inside. This notably applies for channels with $d<1 \mu \mathrm{m}$, since they are proportionally more affected by shrinking or even blocking of the channels' interior during the heating step of fabrication. Furthermore, channels of small widths $d$ are shallower than wider ones [see red triangles in (i)] [59, 60] scaling up the sensitivity to channel closing. The correspondence between the channel depth $h$ and width $d$ is particularly important for these small sized channels. The etch rate $K=h / t$ (etched depth $h$ per time $t$ ) decreases with increasing aspect ratio, meaning that narrower structures are etched at a lower rate. Since the aspect ratio changes during the reactive ion etching (RIE)-step, the etching rate inherently changes during the etching process. This interdependency between the aspect ratio $a$ and the etch rate $K$ (RIE-lag) is attributed to microscopic transport effects within an etched feature [61]. When the length scale of a channel is comparable to - or even smaller than - the mean free path of the particles, their interaction with the channel borders gains importance (Knudsen-transport). Hence, for small channel widths, the flow of etching species to the bottom and of gaseous etch products to the top of the feature is impeded. Therefore the molecules are not only scattered diffusely from the sidewall, but additionally from other particles. However, the first process is predominating [62].
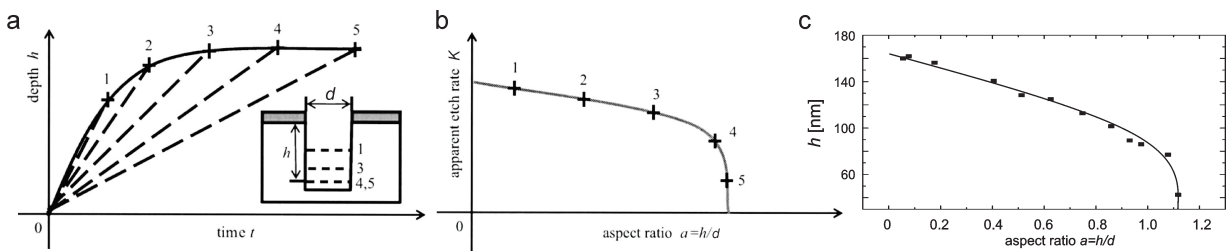

Figure 1.6: Limitation of the channel dimension. (a) Conceptual schematic of the evolution of the depth $h$, of a single silicon trench (channel) as a function of time t. (b) The corresponding apparent etch rate vs aspect ratio curve. Images and caption are reproduced from [59] with adaptions of the nomenclature. (c) The data set presented in Fig. 1.5 (i) of this thesis, in a representation according to (b). As a guide to the eye the data points are connected.

The etch process for a single channel is depicted in Fig. 1.6(a). Since both the supply of etchants and the removal of etching products is diminished for the progressing process, the etching depth $h$ first strongly increases and then gently converges to a certain value (markers 4 and 5); this is equivalently described by the etch rate $K$ decreasing for higher aspect ratios (b). Though (a,b) refer to the etching process of a single channel, these considerations also apply for channels with different width and a constant etching time [60]. Therefore the data in (c) exhibits the same behavior as in (b), especially also revealing a limit for the aspect ratio. Although the RIE-lag is extensively documented for deep plasma etching $(a, b)$, the same mechanisms also affect channels on length scales considered in this work [61].

For a reduced gas pressure the RIE-lag is improved [63], while the etch rate remains constant for changes of the gas pressure up to $\pm 20 \%$ [64]. However, the limitation to an aspect ratio of $a \approx 1.1$ may even be advantageous for waveguide fabrication, leading to square shaped channels entailing a symmetrical mode structure. 
The optical performance of waveguide channels is limited by real structure effects, namely roughness on the channel walls [65]. On the nano-scale, these defective structures also occur during an optimized fabrication process (particularly in the etching step), while waviness on the micron-scale is attributed to imperfections in the lithography step. To a large extent, the first type of roughness is smoothed out by diffusion on the channel surface throughout the high temperature bonding.

Deduced from the findings within this section, there are two different approaches to fabricate "ideal" waveguide channels, supplying a well defined and simultaneously low number of high-intensity modes. At first, channels with a small cross-sectional area instantly provide an exit field consisting of only a few modes. However, the damping - especially pronounced for narrow channels - results in a low intensity. Since a sufficient blocking of the primary beam is required, the channel length cannot be chosen arbitrarily short to reduce the damping. Alternatively, a material with a higher absorption efficiency can be utilized to facilitate short waveguides (see section7). Secondly, longer channels with larger cross-sectional areas may even take advantage of the damping of higher modes during propagating in the channel, resulting also in a low number of modes. Here, an enhanced length is disadvantageous due to the increased interaction of the radiation with real-structure effects. For both approaches smooth waveguide channels are required to achieve intense and symmetric exit fields, since the shape of the intensity distribution inside the channel is significantly affected by sidewall roughness [66]. This challenging fabrication is detailed in section 3 .

\subsubsection{Gratings}

An array of straight waveguides increases the complexity of the wave field inside and outside the channels, offering a structured illumination well suited for reconstruction algorithms.

The FDSs in figure 1.7 depict an array of eleven parallel waveguide channels, each with a width of $d=100 \mathrm{~nm}$, in different spacings $S$. For the simulations identical parameters - for sampling and box sizes - as for single channels were used. In any single guiding channel the wave field is replicated after a certain propagation distance. This longitudinal periodicity is quantified by the inner Talbot-length [see (d)] 67

$$
\frac{L_{T I}}{2}=\frac{\left(2 d_{e}\right)^{2}}{\lambda}
$$

where $d_{e}$ is the effective channel width, comprised of the channel width $d$ measured by an SEM tool and the penetration depth $d_{p}$ [68], such that $d_{e}=d+2 \cdot d_{p}$ [69]. For $E_{p h}=7.9 \mathrm{keV}$, the penetration $\operatorname{depth}^{3} d_{p}=\lambda /\left(2 \pi \theta_{c}\right)$ is $6.3 \mathrm{~nm}$. Every $n \cdot L_{T I} / 2$ (with $n \in 2 \mathbb{N}$ ) interval, the intensity pattern is replicated inside the channel, resulting in $81 \mu \mathrm{m}$ for the first self-imaging distance. From a line profile through $z=0$, a value of $83 \mu \mathrm{m}$ is found to be in good agreement with the theoretical prediction [see (d)]. A representative analysis is carried out on the topmost and central channel.

$\overline{3}$ Derived from the general expression $d_{p}=\frac{\lambda}{2 \pi \sqrt{\sin ^{2} \theta_{c}-\sin ^{2} \theta}}$ for a beam parallel to the optical axis $\left(\theta=0^{\circ}\right)$. 


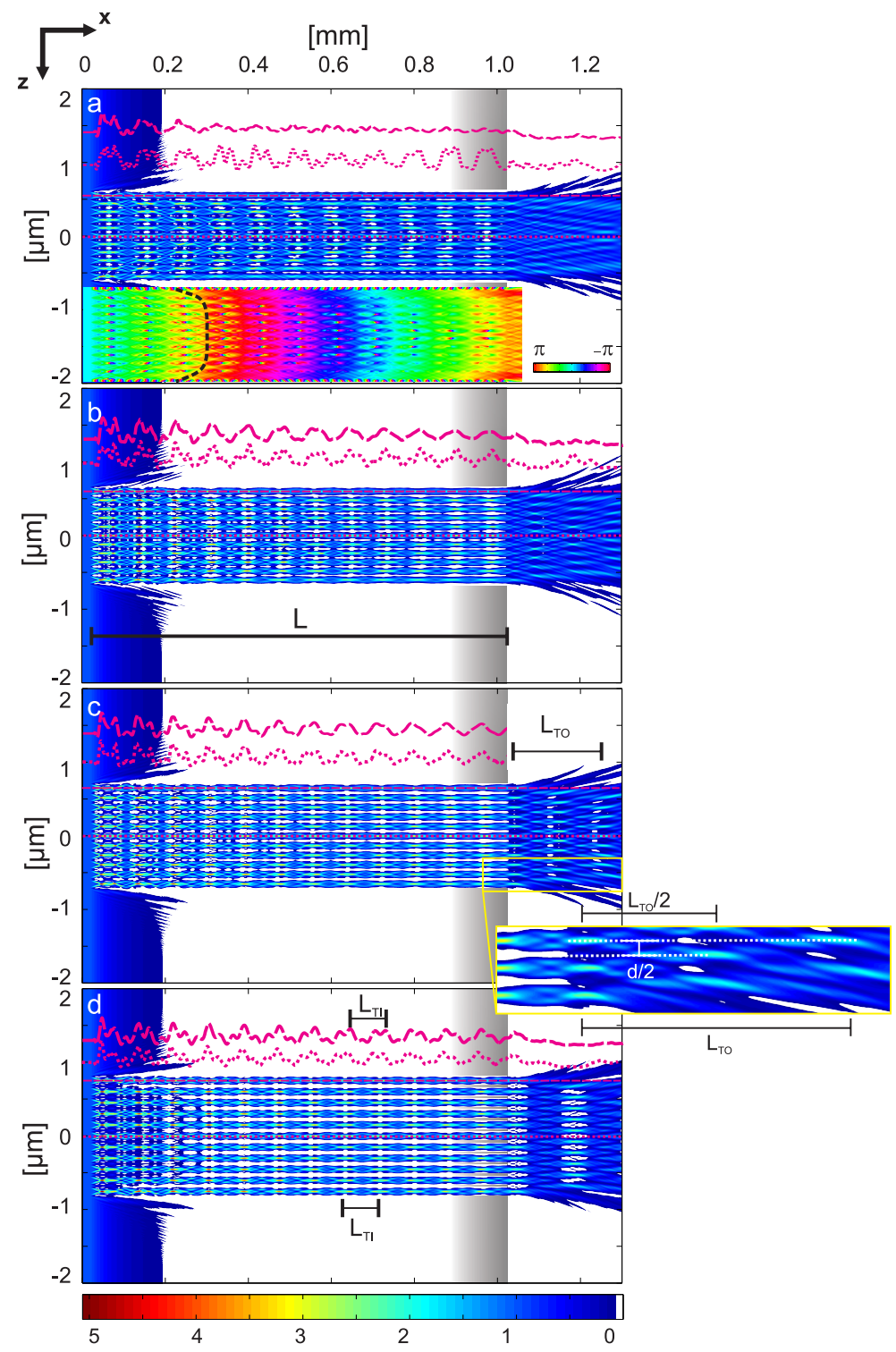




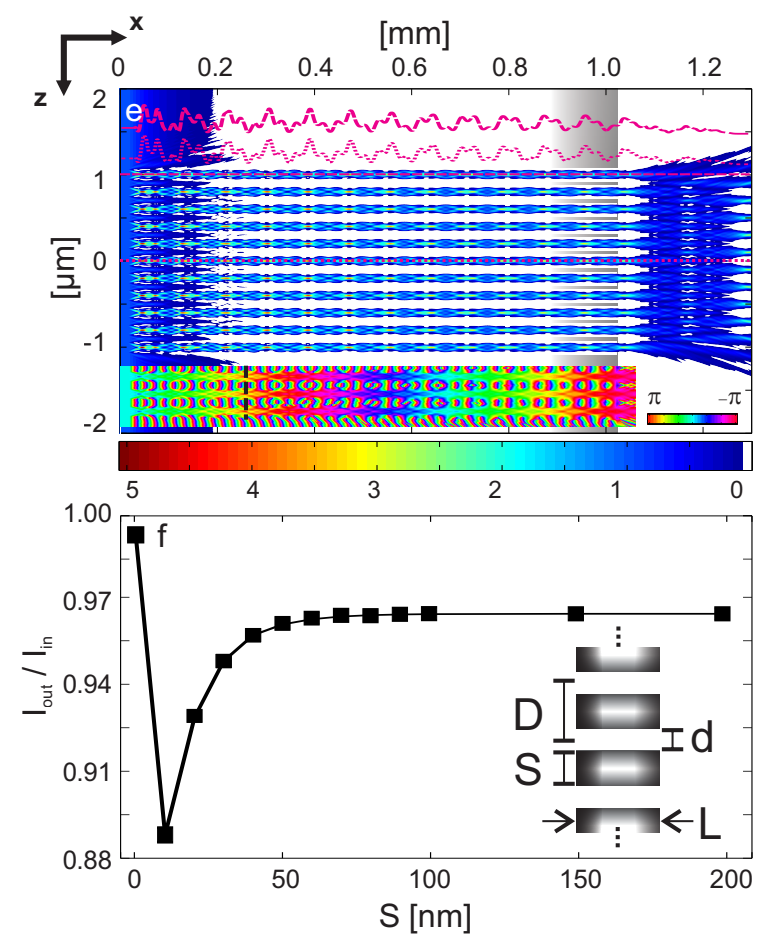

Figure 1.7: Guiding properties of $2 D$ waveguide gratings. FDSs of arrays of 11 parallel channels each with a diameter of $100 \mathrm{~nm}$ and different spacings $S$ of (a) $10 \mathrm{~nm}$, (b) $20 \mathrm{~nm}$, (c) $30 \mathrm{~nm}$, (d) $50 \mathrm{~nm}$ and (e) $100 \mathrm{~nm}$. Intensity profiles in $x$-direction are plotted in magenta, while the upper one belongs to the topmost channel (dashed) and the second one refers to the central channel of the array (dotted). The phases are depicted for the whole structure in (a) and for the 3 bottommost channels in (e), all in units of radian. (f) The $I_{\text {out }} / I_{\text {in }}$ ratio as a function of the channel spacing $S$. All intensities are normalized to the input intensity.

Although the replicated intensity pattern is originally a single-channel phenomenon, for a grating with a small spacing of $S=10 \mathrm{~nm}$ (a), the profiles from the two compared channels significantly differ in amplitude and shape. The field of the central channel deviates from an undisturbed channel, shown in (e) where the number of replications is reduced. This modification corresponds to the deviation in the phase of the outermost channels, were the phase is "lagging behind", referring to the central channel. This is visualized by black dotted lines, connecting the same phase values [compare (a) and (e)]. In (e) the absolute value of the phase change along the channel is $\approx 2.5 \cdot \pi$, while in (a) this value is not accomplished for neither of the channels. Furthermore, central channels are apparently less affected by unsymmetrical intensity distributions than the outer ones [see (a)-(e)], indicating that the observed phenomenon is attributed to overlapping fields of the neighboring channels. With increasing spacing $S$ the impact of overlapping fields decreases (b-c). For $S \geq d / 2$ the field is similar to the single channel case (d), such that the cross talk between the channels is not dominating the wave field anymore. 
Without any spacing $(S=0 \mathrm{~nm})$, the $I_{\text {out }} / I_{\text {in }}$ ratio corresponds to a $1100 \mathrm{~nm}$ wide channel. As noticed for the phase, the propagation is disturbed by the cross talk, such that the exit intensity reveals a minimum for the smallest spacing $S=10 \mathrm{~nm}$ [see (f)], which is also evident due to the decreased amplitudes of the profiles. When the impact of the neighboring channels decreases with increasing spacing, the intensity converges to the value of a single straight $d=100 \mathrm{~nm}$ wide channel [compare Fig. 1.3(h)].

Analogously to the replications of the wave field inside the channel, a noticeable feature can be observed in the near-fields of Fig. 1.7(c-e). The field distribution of the exit plane is also replicated after a certain propagation distance, in this case as a function of the distance $D$ between two neighboring channel centers. The relation between channel width $d$, spacing $S$ and distance $D$ is: $D=S+d$. The position of the replicated image is described by the outer Talbot-length $L_{T O}$ [see (c)], given by

$$
\frac{L_{T O}}{2}=\frac{D^{2}}{\lambda} \text { assuming that } D \gg \lambda=0.157 \mathrm{~nm} .
$$

Likewise, the self-image is replicated after odd integer multiples of $L_{T O}$. For a distance of $D=30 \mathrm{~nm}$ the Talbot-length $L_{T O}$ is $215 \mu \mathrm{m}$, which is found to be in good agreement to the value of $213 \mu \mathrm{m}$ estimated from (c). Furthermore, the interference maximum at $L_{T O} / 2=108 \mu \mathrm{m}$ can is identified at a distance of $106 \mu \mathrm{m}$ as well. The interference pattern of the monochromatically illuminated diffraction grating is shifted by $d / 2$ in $z$-direction, such that the maxima are located exactly between two channels, as expected [70] [see inset in (c)].

Waveguide gratings were experimentally characterized at the beamline BM20 [European Synchrotron Radiation Facility (ESRF, France)] at $E_{p h}=11.5 \mathrm{keV}$. At $3.5 \mathrm{~m}$ behind the waveguide device, the far-field patterns were recorded by a Pilatus 100k detector (Dectris).

In figure 1.8 (a), the transmission $T$ of different gratings is depicted. These channel arrays form pairs with approximately the same widths $d$ but two different spacings $S$; here, the channels with smaller spacings are generally 5-10\% wider. The transmission of a grating is calculated in the same way as for single channels, namely by comparing the transmitted to the incoming intensity. To estimate the latter, the ratio of beam size and incoupling area has to be considered; for gratings this is equal to the sum of the entrance areas of the 20 single channels. Taking this into account, the transmission for equally sized gratings is constant when $S$ is enlarged, as expected for spacings $S>100 \mathrm{~nm}$ [see Fig. 1.7(f)]. The optical performance of 20 channels each with $d=150 \mathrm{~nm}$ [black circles in Fig. 1.8(a)] can hence be compared to a single channel of a width $d=3 \mu \mathrm{m}$ that was located on the same waveguide device. The single channel revealed a transmission of $T=21 \%$ [see (b)], which is in good agreement to the average value of $T=23 \%$, estimated for the two gratings. Therefore, the grating geometry reveals equally good transmission properties, while additionally providing a structured illumination for particular $\mathrm{x}$-ray imaging applications. The expected transmission value of $T=96 \%$ [see Fig 1.7 (f)] is not reached due to losses of intensity, attributed to scattering at real-structure effects or absorption. 

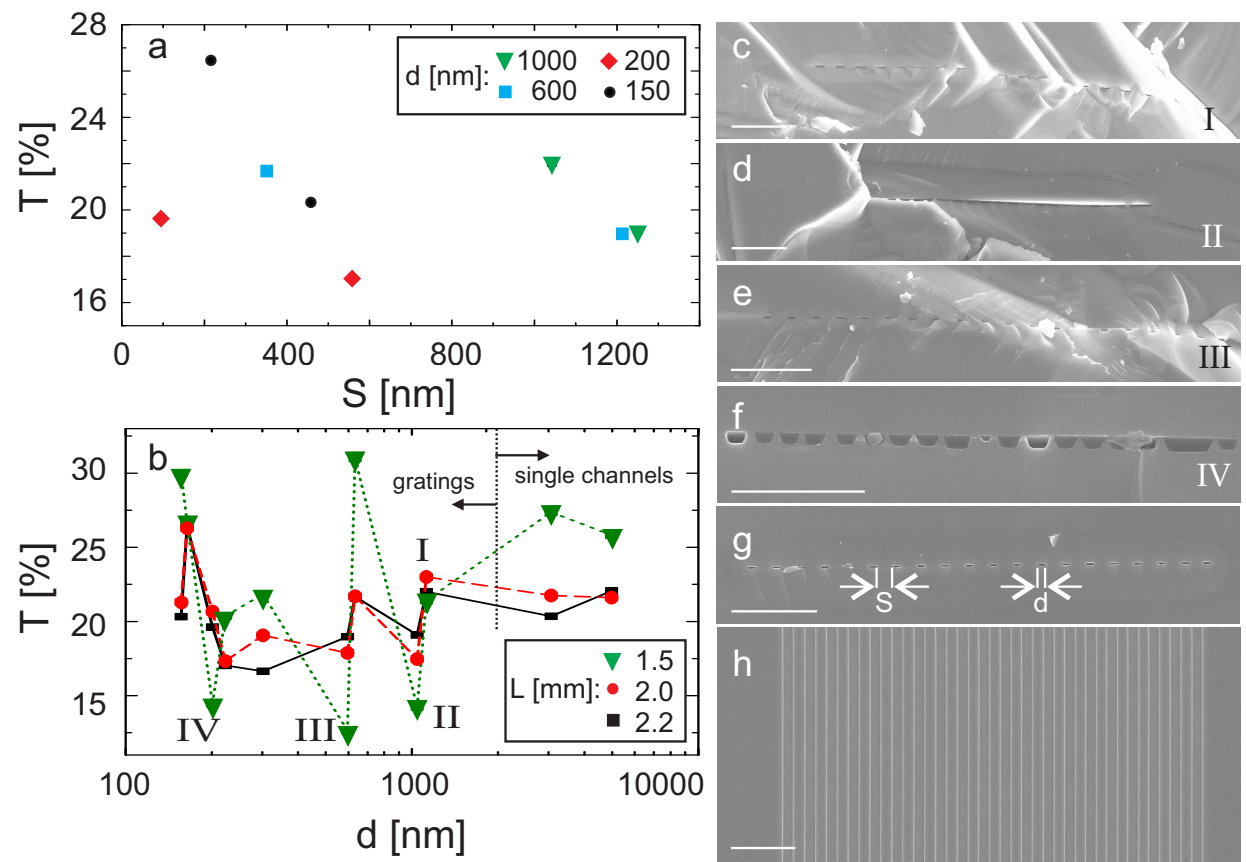

Figure 1.8: Experimental results for $2 D$ waveguide gratings. (a) Transmission values of arrays of 20 channels with different spacings $S$ and widths $d$ of $1000 \mathrm{~nm}$ (green triangle), $600 \mathrm{~nm}$ (blue square), $200 \mathrm{~nm}$ (red diamond) and $150 \mathrm{~nm}$ (black circle). (b) Transmission values as a function of the channel width $d$ and the length L: $1.5 \mathrm{~mm}$ (green triangle), $2.0 \mathrm{~mm}$ (red circle) and $2.2 \mathrm{~mm}$ (black square). (c-f) SEM micrographs of the front side of gratings revealing severe degradation and defects. The channel gratings could not be used for experiments. (g) Front side view on a fully functional grating. (h) Top view of an array. Scale bars denote $0.5 \mu \mathrm{m}$.

In figure 1.8(b), the transmission for several devices with identically sized gratings and single channels in different lengths of $1.5 \mathrm{~mm}$ (green triangle), $2.0 \mathrm{~mm}$ (red circle) and $2.2 \mathrm{~mm}$ (black square) are compared. As discussed above, a shortened optical path length results in an enhanced performance, due to the reduced impact of realstructure defects. The few exceptions from this general trend - marked with I-IV - are attributed to damages in the respective structures (c-f). These grating are deformed or the channels are not equally sized, both causing loss of intensity. The SEM inspection served to detect such severe flaws and to improve the fabrication, resulting in fully functional channels, for instance (g) is the well performing version of (e). An example for a grating with $S=d$ is shown in (h). This SEM micrograph was taken during the production process in the unbond state, giving an impression about the accuracy of the fabrication technique, described in detail in chapters 2 and 3 The corresponding far-field patterns are considered as representative examples in section 1.3 


\subsubsection{Tapered channels}

The main advantage of tapered waveguides with respect to straight channels is an increased intensity. While only a fractional amount of the incoming beam is transmitted by the straight channel, an approximately ten times larger part can be funneled down to the same exit size, hence resulting in an enhancement of the intensity by an order of magnitude.

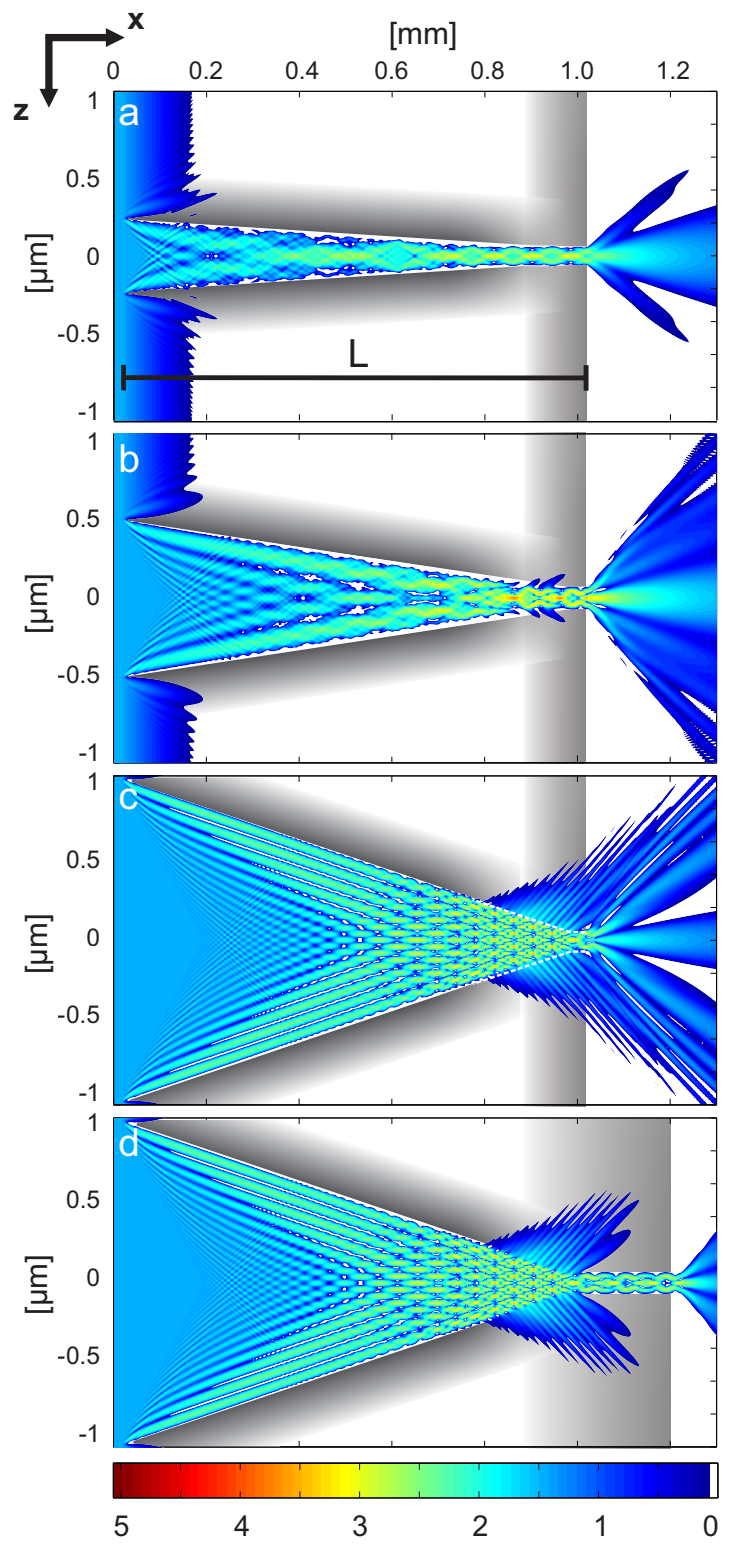




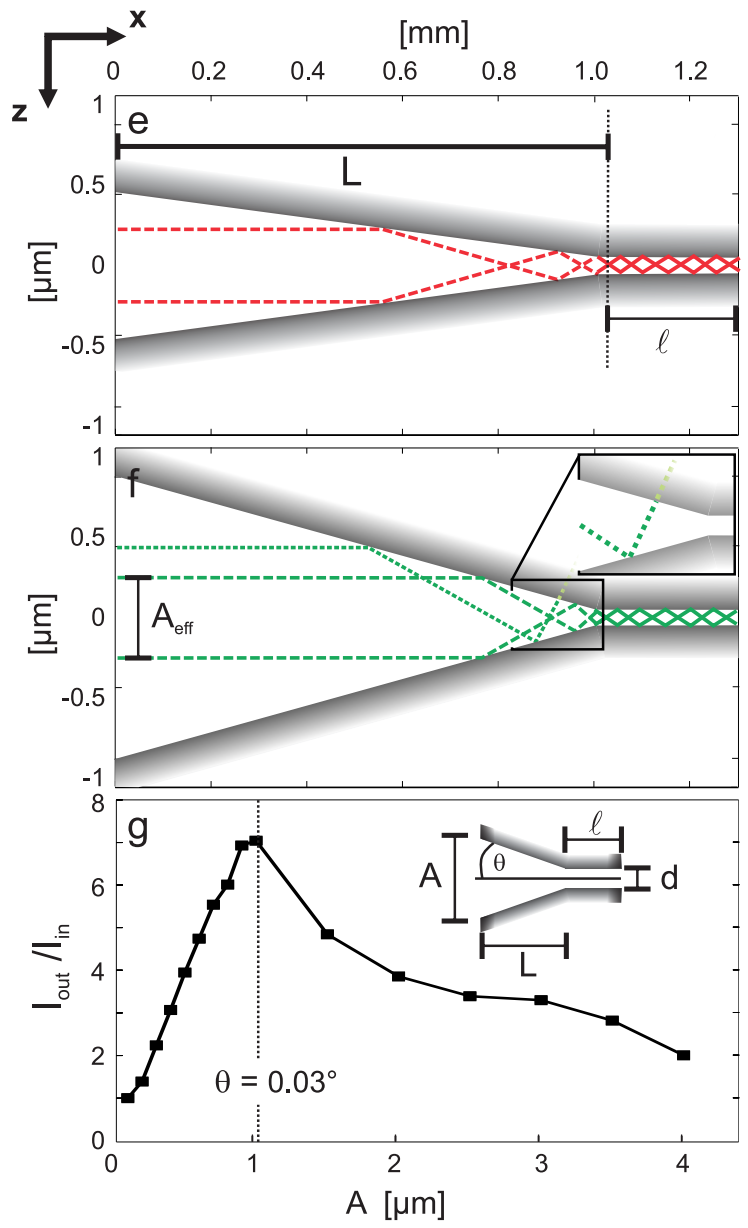

Figure 1.9: Guiding properties of tapered waveguides. FDSs of tapered waveguides with different entrance sizes $A$ of (a) $0.5 \mu \mathrm{m}$, (b) $1 \mu \mathrm{m}$ and (c) $2 \mu \mathrm{m}$. (d) Channel with an entrance size of $A=2 \mu \mathrm{m}$ and an increased length of $\ell=0.2 \mathrm{~mm}$. (e) Confinement of the wave field in a tapered channel with an entrance size of $A=1 \mu \mathrm{m}$. (f) Illustration of loss mechanisms connected to the opening angle $\theta$, using the example of an $A=1.5 \mu \mathrm{m}$ wide channel. (g) The $I_{\text {out }} / I_{\text {in }}$ ratio as a function of entrance size A. All intensities are normalized to the input intensity.

In figure 1.9 FDSs of tapered channels with an entrance size $A$ up to $2 \mu \mathrm{m}$ are depicted for a channel length of $L=1 \mathrm{~mm}(\mathrm{a}-\mathrm{c})$, whereas parameters identical to straight channels were chosen. The incoming radiation impinges on the channel border at an angle $\theta$ and is reflected (possibly several times) while propagating within the channel. This way, the wave field is concentrated and the point of maximum intensity is no longer located near the entrance at $x=0$, but is shifted in positive $x$-direction towards the exit side. At an entrance size slightly above $1 \mu \mathrm{m}$ (b), the focal spot of the tapered channel is positioned directly in the exit plane, resulting in a maximum $I_{\text {out }} / I_{\text {in }}$ ratio (g). When the opening angle exceeds the value of $\theta=0.03^{\circ}$, the intensity at the channel 
exit decreases. In (e) and (f) this situation is illustrated graphically. For the channel with $A=1 \mu \mathrm{m}$ (e), the confinement of the beam results in a focal spot in the exit plane. This focus at $x=L$ can either directly serve as a nano-sized $\mathrm{x}$-ray source, or the beam can be guided by another channel, as depicted here. For $A=1.5 \mu \mathrm{m}$ (f), only the inner third ( $A_{\text {eff }} \leq \pm A / 6$ relative to the optical axis at $z=0$ ) of the channel contributes to the beam that is coupled into the straight channel behind the tapered region. When radiation impinges at the region of the channel near the borders at $\pm A / 2$, the critical angle $\theta_{c}=0.227^{\circ}$ is exceeded after a few reflections causing x-rays to enter the cladding [see (c) and the inset in (f)]. The larger $A$ becomes, the smaller is the inner part of the taper that guides the field and with it the intensity of the exit field. Since this leakage occurs near the exit side, most of the radiation propagating in the cladding is not fully absorbed and appears in the form of radial symmetric ring-like structures in the far-field patterns [see Fig. 1.14 1.16 , Chap. 5. Fig. S3 and also for straight channels Fig. 1.5 (g and $\left.\mathrm{h}_{1}\right)$ ]. Although this radiation is attenuated, the disturbances in the far-field cannot be neglected especially regarding their impact on the reconstruction of the field in the exit plane of the waveguide.

Due to scattering processes, the focal spot is extended to an area, which is possibly even divided into unconnected sections. However, a successful and reliable reconstruction of the waveguide exit field (for example by the well known error reduction algorithm [14]) requires a compactly supported and well defined radiation source. Furthermore, the radiative modes carry an arbitrary phase distribution making the phase retrieval a more challenging task. By adding a straight channel section of length $\ell$ at the exit plane of the taper at $x=L$ [see Fig. $1.9(\mathrm{~d})$ and (e)], the scattered intensity is absorbed by the additional cladding. Associated with this channel extension, the damping and scattering of radiation due to surface imperfections have to be considered in waveguide fabrication. Thus, on the one hand, the straight part needs to be as short as possible; on the other hand, for a reasonable attenuation $\left(T \approx 10^{-7}\right)$, a sufficient length is required, depending on the photon energy. Hence, a compromise has to be made by choosing the length $\ell$, which is adapted to the specific experimental conditions.

Next, experimental results for tapered channels are presented (a detailed description of the reconstruction procedure is given in section 1.3. The channel characterized in figure 1.10 was tested at the P10 beamline at PETRA III, DESY, at $E_{p h}=7.9 \mathrm{keV}$. Exhibiting a length of $L=0.8 \mathrm{~mm}$, the channel is narrowed down from $319 \times 142 \mathrm{~nm}^{2}$ at the entrance to $98 \times 83 \mathrm{~nm}^{2}$ at the exit side. In accordance with the decreased width $d$, the channel is shallower at the exit side [see 1.5(i)].

Apart from the very good agreement referring to the shape of the intensity pattern and the small percentage deviation of $1 \%$ between reconstruction and measured data, the intensity values reconstructed at the gaps between the detector modules are an evidence for a high reliability of the retrieved solution (see Fig. $1.10(a, b)$ ). The intensity distribution in the exit plane of the channel (c) exhibits a maximum in intensity in a central left position, equipped with an approximately flat phase (d). The region around the peak, marked with a dotted black line, is presented in an enlarged view in (e). Profiles are extracted across the maximum and fitted with a Gaussian function, yielding values of $23 \times 24 \mathrm{~nm}^{2}$ (FWHM), hence facilitating a virtual point-source with an intensity of $I=1.45 \cdot 10^{10} \mathrm{cps}$ corresponding to a transmission of $T=40 \%$, suit- 
able for high resolution x-ray imaging. Although the ring-like features arising from scattered but only partially absorbed radiation are still visible, their intensity is considerably reduced. Furthermore, neither higher focusing orders nor pronounced tails of pre-focusing optics are apparent, which leads to achieving a low background signal.

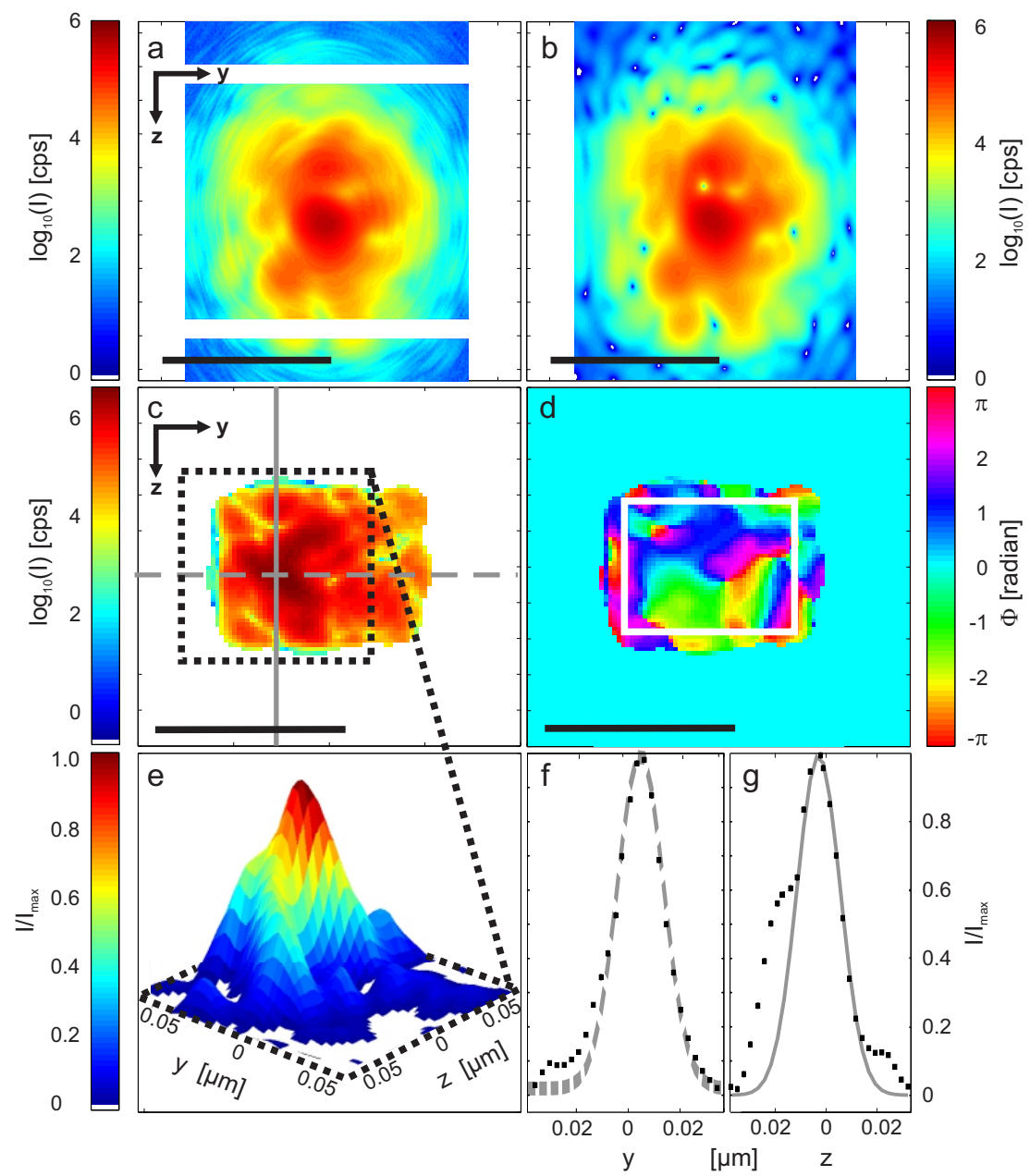

Figure 1.10: Characterization of a tapered channel with an exit size of $d=98 \mathrm{~nm}$ and $\mathrm{h}=83 \mathrm{~nm}$. (a) Measurement and (b) reconstruction of the far-field pattern. Scale bars denote $50 \mathrm{~mm}$. (c) Reconstructed amplitude and (d) phase $\Phi$, with a white square marking the initial support. Scale bars denote $100 \mathrm{~nm}$. (e) Enlarged view of (c). (f) Horizontal and (g) vertical intensity profiles from (c) as black dots and Gaussian fits in gray, both normalized to the maximum intensity of $(c)$. 


\subsubsection{Curved channels}

Curved channels enable guiding $x$-ray beams around a bent, for example offering precisely delayed pulses or reference beam measurements.

Considering the theoretical background, the Helmholtz equation (1.4) can be rewritten for the straight channel case, using Eq. 1.8:

$$
\frac{\partial^{2} \Psi(z)}{\partial z^{2}}+\underbrace{\left(4 \pi r_{0} \rho(z)\right)}_{=: P_{S}} \Psi(z)=\underbrace{\left(\beta_{\mathrm{d}}^{2}-k^{2}\right)}_{=M} \Psi(z) \text { with } \rho(z)= \begin{cases}0 & \text { for } \quad d \geq z \geq 0 \\ \rho_{\mathrm{Si}} & \text { else. }\end{cases}
$$

As stated above, the eigenvalues of waveguide modes correspond to the energy levels of an electron trapped in a potential well of height $P_{s}$. For the simulation of curved waveguides, two complementary strategies were pursued. Firstly, a "real" curvature was realized by converting the straight trajectory of the index of refraction $n(z)$ into a curved line, such that $n(x, z)$ and also $\rho(x, z)$. Secondly, an "artificial" curvature was implemented in a straight guiding layer, by modifying the value of $n(z)$, adding an adjusted linear potential $P_{c}[71,72$, defined by

$$
\frac{\partial^{2} \Psi(z)}{\partial z^{2}}+\underbrace{\left(4 \pi r_{0} \rho(z)-\frac{2 k^{2}}{R} z\right)}_{=: P_{c}} \Psi(z)=\left(\beta_{\mathrm{d}}^{2}-k^{2}\right) \Psi(z)
$$

The curved waveguide is assumed to be a section of a circular arc with $R$ being the radius of curvature [see Fig. 1.11(e)], pointing in $r$-direction (unit vector $e_{r}$ ).

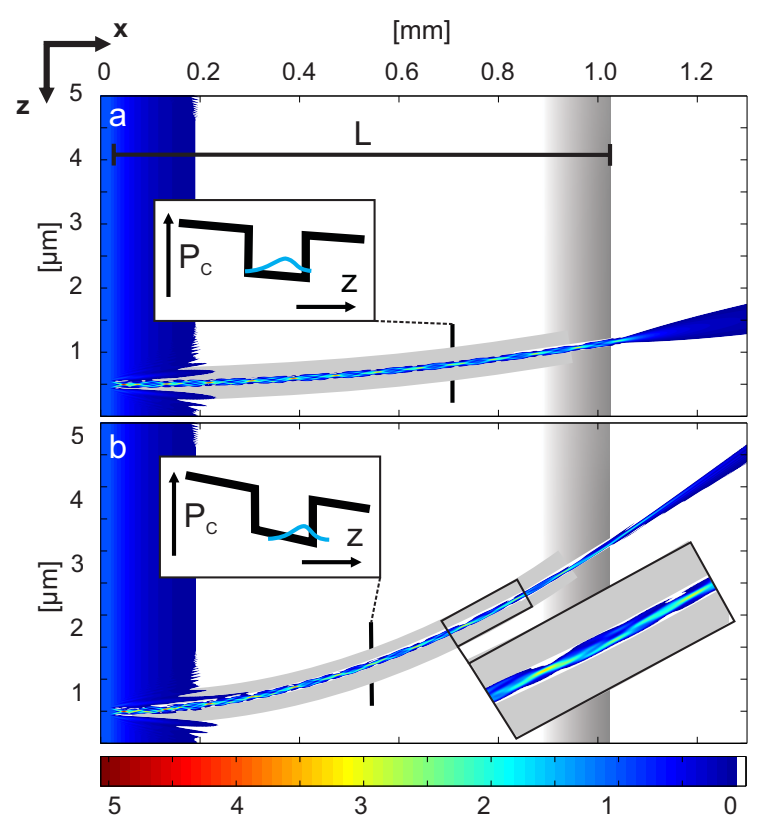



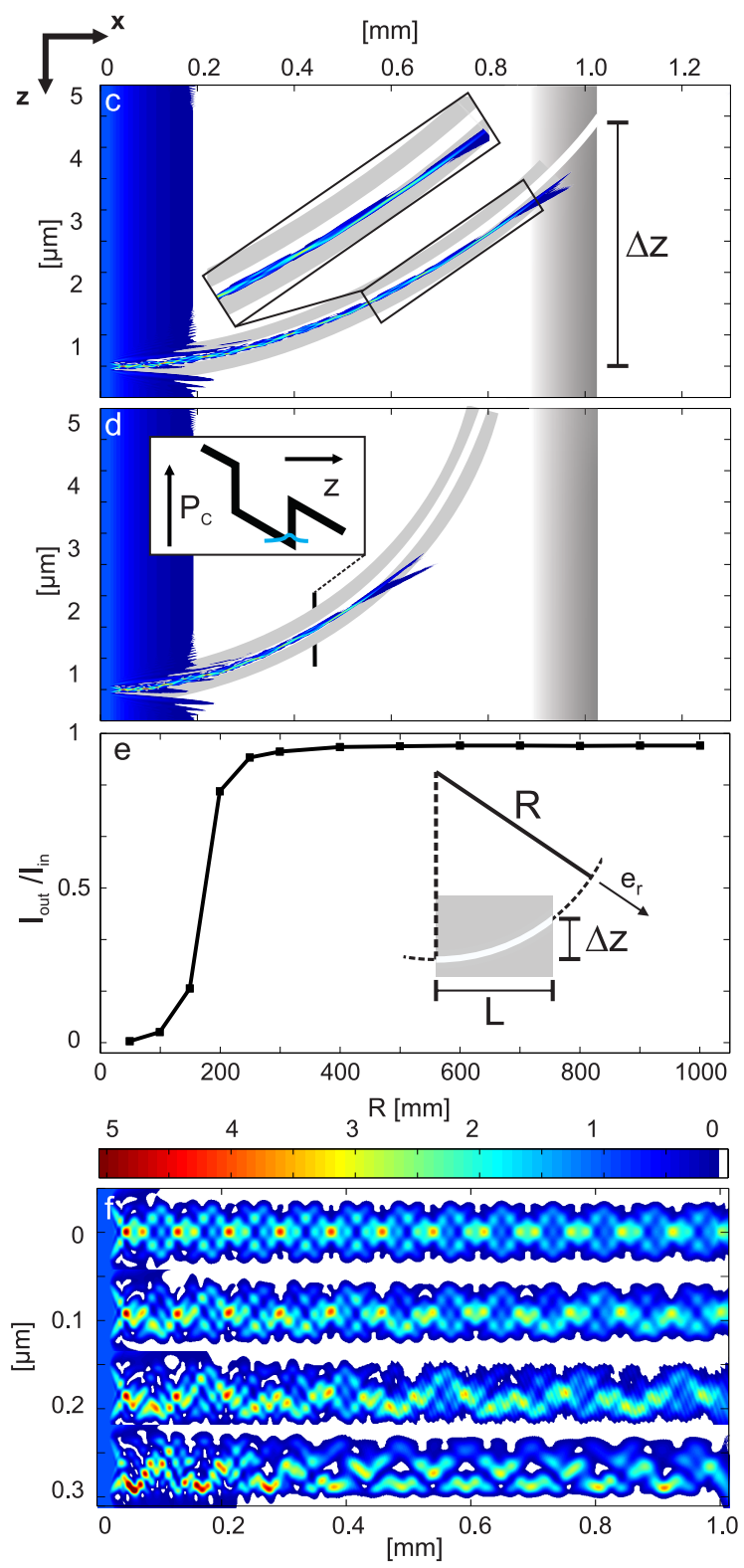

Figure 1.11: Guiding properties of curved waveguides. FDSs of $d=100 \mathrm{~nm}$ wide, curved channels with different radii of curvature $R$ of (a) $800 \mathrm{~mm}$, (b) $200 \mathrm{~mm}$, (c) $150 \mathrm{~mm}$ and (d) $100 \mathrm{~mm}$. (e) The $I_{\text {out }} / I_{\text {in }}$ ratio as a function of the curvature R. (f) FDSs of straight waveguide channels with $d=100 \mathrm{~nm}$, including the linear gradient in the refractive index in z-direction. The gradient corresponds to $R: 800 \mathrm{~mm}, 200 \mathrm{~mm}, 100 \mathrm{~mm}$ and $25 \mathrm{~mm}$, from top to bottom. All intensities are normalized to the input intensity. 
The simulation parameters were chosen according to the straight channels, despite of an enlarged box size of $10 \mu \mathrm{m}$ in the $z$-direction with a sampling rate of $1 \mathrm{~nm} / \mathrm{px}$. In figure 1.11 (a-d) curved channels with different radii $R$ in the interval $[800 \mathrm{~mm}, 200 \mathrm{~mm}$, $150 \mathrm{~mm}, 100 \mathrm{~mm}$ ] are depicted. The inlays illustrate the corresponding potential $P_{c}$ steepening with decreasing curvature $R$. At first, the channel geometry corresponds to a straight waveguide, also in terms of intensity. When $R$ is decreased, the modes are shifted towards the outer region of the channel $(r>R)$, responding to the minimum in potential [see enlarged view in (b)]. This behavior is well known from whispering gallery modes [73, 74]. At even lower curvatures, only a small amount of the radiation that was coupled into the waveguide in the first place is guided through the device by the channel; the main part is absorbed in the cladding. Finally, the field is exclusively propagating in the cladding. This leaking process is displayed in the enlarged view in (c). In (e) the $I_{\text {out }} / I_{\text {in }}$ ratio is plotted as a function of $R$, showing the transition between the guided modes and the evanescent field. Note that the steep incline is not attributed to the actual loss mechanism, but is a numerical artifact. The simulations were performed using the paraxial wave equation, offering a limited applicability in calculating the internal field within a waveguide, since this approximation is only valid for slight changes of the direction of the radiation. For the curvatures depicted here this requirement is no longer fulfilled. Therefore, the loss of the guided modes seems to arise suddenly while in reality this continuous process is characterized by a smooth transition. For this reason the illustrations in figure $1.11(\mathrm{a}-\mathrm{d})$ are adequate to describe the propagation of a field in a curved channel just within certain limits 4

This issue is circumvented by implementing the potential $P_{c}$ in the simulations (f). The index of refraction $n$ of the guiding layer (vacuum) is modified by adding a linear gradient in $z$-direction in the order of $10^{5}$, corresponding to curvatures $800 \mathrm{~mm} \geq$ $R \geq 25 \mathrm{~mm}$. According to the positive $r$-direction for curved channels, the field distribution and especially the regions containing high intensity are notably displaced towards positive $z$-direction. As mentioned above, the field can be guided at much smaller curvatures; actually channels with radii as small as $R<3.5 \mathrm{~mm}$ still show a waveguiding effect. This is exemplarily depicted in the appendix together with a template for the simulations.

At beamline ID01, ESRF at $E_{p h}=8 \mathrm{keV}$, channels with a length of $L=850 \mu \mathrm{m}$ and curvatures of $700 \mathrm{~mm} \geq R \geq 200 \mathrm{~mm}$ were tested. The channels revealed sizes of (a) $212 \mathrm{~nm}$ $\times 127 \mathrm{~nm}$, (b) $65 \mathrm{~nm} \times 60 \mathrm{~nm}$, (c) $132 \mathrm{~nm} \times 114 \mathrm{~nm}$, (d) $114 \mathrm{~nm} \times 127 \mathrm{~nm}$ and (e) $109 \mathrm{~nm}$ $\times 109 \mathrm{~nm}(d \times h)$. In figure 1.12 (a-e), far-field patterns are displayed, recorded by a Maxipix detector (ESRF) at a distance of $1 \mathrm{~m}$ behind the waveguide device. The primary beam appears as a bright spot at a fixed position near the bottom. The intensity patterns exhibiting the typical waveguide shape (compare to figure 1.5 ), were recorded for channels down to $R=200 \mathrm{~mm}$, which corresponds to a deflection up to $0.6^{\circ}$. In (e), the arrow indicates the theoretical deflection, which is found to be in good agreement with the center of the waveguide pattern. In addition to the mechanisms described above, the intensity decreases even more with $R$, since the length of the channel is increased and therefore also the impact of real-structure defects, such as roughness on

4 In Appendix B a new implementation with a rotating coordinate system is depicted, solving this problem. 
different length scales, leading to an enhanced scattering at the boundaries [66]. The curvature $R$ of a certain channel was calculated from its entrance and exit positions in $z$-direction, relative to the edge of the waveguide device [see Fig. $1.11(\mathrm{c}, \mathrm{e})]$ and the length of the device $L$, using the expression $R=\left(4 \Delta z^{2}+L^{2}\right) /(8 \Delta z)[75$.

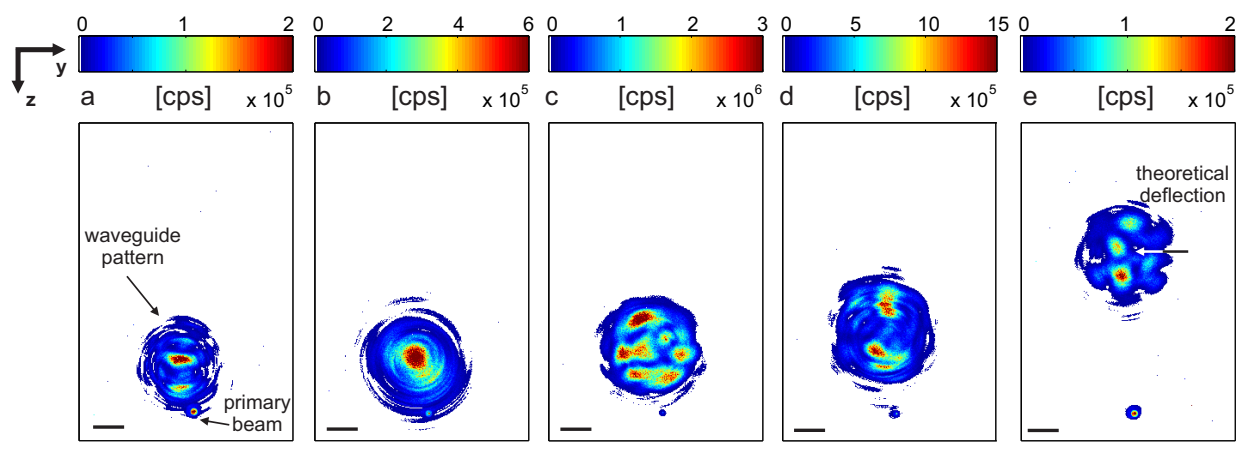

Figure 1.12: Experimental results for curved waveguides. Recorded far-field patterns of waveguide channels with curvatures $R$ of (a) $700 \mathrm{~mm}$, (b) $600 \mathrm{~mm}$, (c) $500 \mathrm{~mm}$, (d) $400 \mathrm{~mm}$ and (e) $200 \mathrm{~mm}$. The theoretical deflection, is exemplarily depicted in (e), marked by an arrow. Scale bars denote $2 \mathrm{~mm}$, intensities are given in counts per second.

\subsubsection{Beamsplitters}

Splitting one $x$-ray beam into two sub-beams allows using waveguides as advanced optical tools, for example as interferometers or for off-axis holography with coherent beams. In this section, waveguides in a beam-splitting geometry are characterized by FDSs, while experimental results are presented in chapter6. For the FDSs, the same parameters as for straight channels were chosen, except for the $z$-direction, where $15 \cdot 10^{3}$ pixels cover $15 \mu \mathrm{m}$. Figure 1.13 shows a single waveguide channel with a width of $d=200 \mathrm{~nm}$ that is split into two channels, each with a width of $d=100 \mathrm{~nm}$. Contrarily to straight structures, the field distribution inside any of the split channels reveals a mirror-symmetry referring to the axis along the channels center - marked by dashed lines in the insets in (a) and (b). Furthermore, the regions of high intensity exhibit an alternating movement from the left to the right channel border in the direction of the channel axis; this is illustrated with a solid, magenta line in (e). Starting with $\alpha=0^{\circ}$, the channel geometry corresponds to a $d=200 \mathrm{~nm}$ wide straight waveguide, which applies also for the $I_{\text {out }} / I_{\text {in }}$ ratio [compare Fig. 1.3 (i) and $\left.1.13(\mathrm{f})\right]$. For small opening angles $\alpha>0^{\circ}$ the two channels are separated and the intensity ratio is equal to the value for a straight channel with a width of $d=100 \mathrm{~nm}$. While increasing the angle further (b-e), the intensity of the exit field decreases continuously. This is not only attributed to losses at the point where the input-channel is split, but also to the increased absorption due to the enlarged optical path length. When the critical angle $\theta_{c}$ is exceeded $\left(\alpha>0.227^{\circ}\right)$, the radiation is no longer reflected at the channel boundaries, but propagates in the cladding; as discussed in section 1.2 .3 Therefore the $I_{\text {out }} / I_{\text {in }}$ ratio drops to zero. 


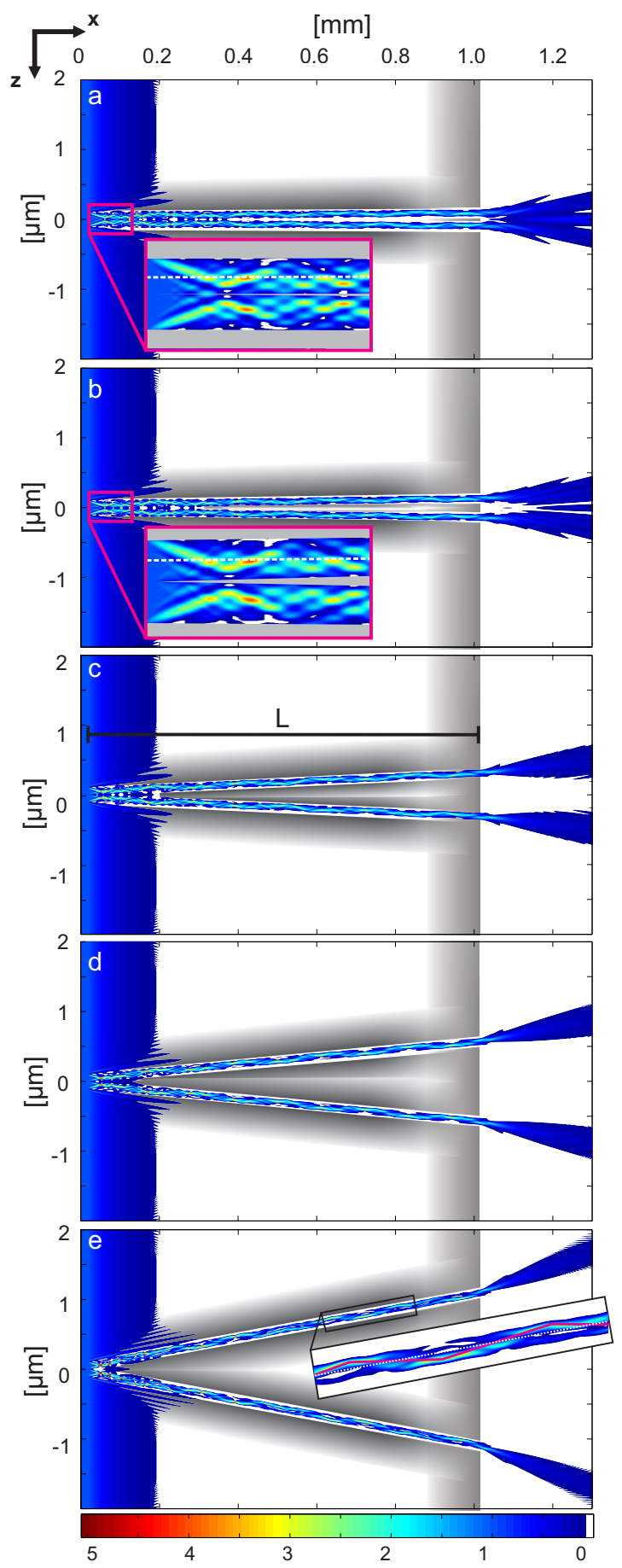




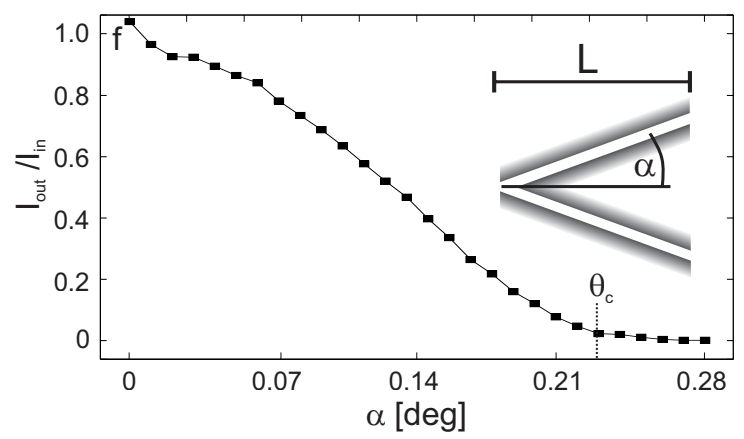

Figure 1.13: Guiding properties of a waveguide beamsplitter. FDSs for different opening angles $\alpha$ of (a) $2.5 \mathrm{mdeg}$, (b) $5 \mathrm{mdeg}$, (c) $15 \mathrm{mdeg}$, (d) $30 \mathrm{mdeg}$ and (e) $60 \mathrm{mdeg}$. ( $f$ ) The $I_{\text {out }} / \mathrm{I}_{\text {in }}$ ratio as a function of the opening angle $\alpha$. All intensities are normalized to the input intensity.

\subsection{Probe reconstruction}

Waveguides serve as quasi point-sources for $x$-rays, since the wave field is highly confined in two dimensions. Due to this property, they are well suited for high resolution far-field $x$-ray imaging.

In order to reconstruct the near-field intensity distribution from the recorded far-field, the error reduction (ER) algorithm [14] is used. An iterative loop of discrete Fourier back and forth transformations (DFT and inverse DFT, respectively) between the exitplane of the waveguide chip and the detector is used to retrieve the original illumination from the measured far-field data, while two constraints are applied on the reconstructed wave field. At first, the field at the waveguide exit has to be limited to a predefined area (support constraint). The size of this area (support) is estimated from SEM micrographs of the channels exit and then enlarged by $5 \mathrm{~nm}$ in each direction, corresponding to the penetration depth $d_{p}=6.3 \mathrm{~nm}$ (see section 1.2 .1 . Secondly, the reconstructed amplitudes in the detector plane are adapted to the measured intensities (magnitude constraint).

For each data set, 1000 iterations were performed to ensure a sufficient convergence of the reconstruction. This is evidenced by a constant percentage deviation $(<2 \%)$, calculated by comparing the measured intensity distribution to the reconstructed farfield pattern. In the last iteration step the support constraint was modified using the shrink wrap procedure [76]. In this way, the support is not limited to the initial condition/area; namely all regions carrying more than $5 \%$ of the maximum intensity are included. Hence, non-negligible intensity values may be reconstructed in areas exceeding the initial support, marked with white squares. This relaxation of the constraint is performed to check the plausibility of the reconstructed near-field, especially whether the support is divided into disconnected areas.

In this section, reconstructions for several straight channels and a grating are presented. Examples for waveguides in tapered and beam splitting geometry can be found in chapters 4 and 6 respectively. 


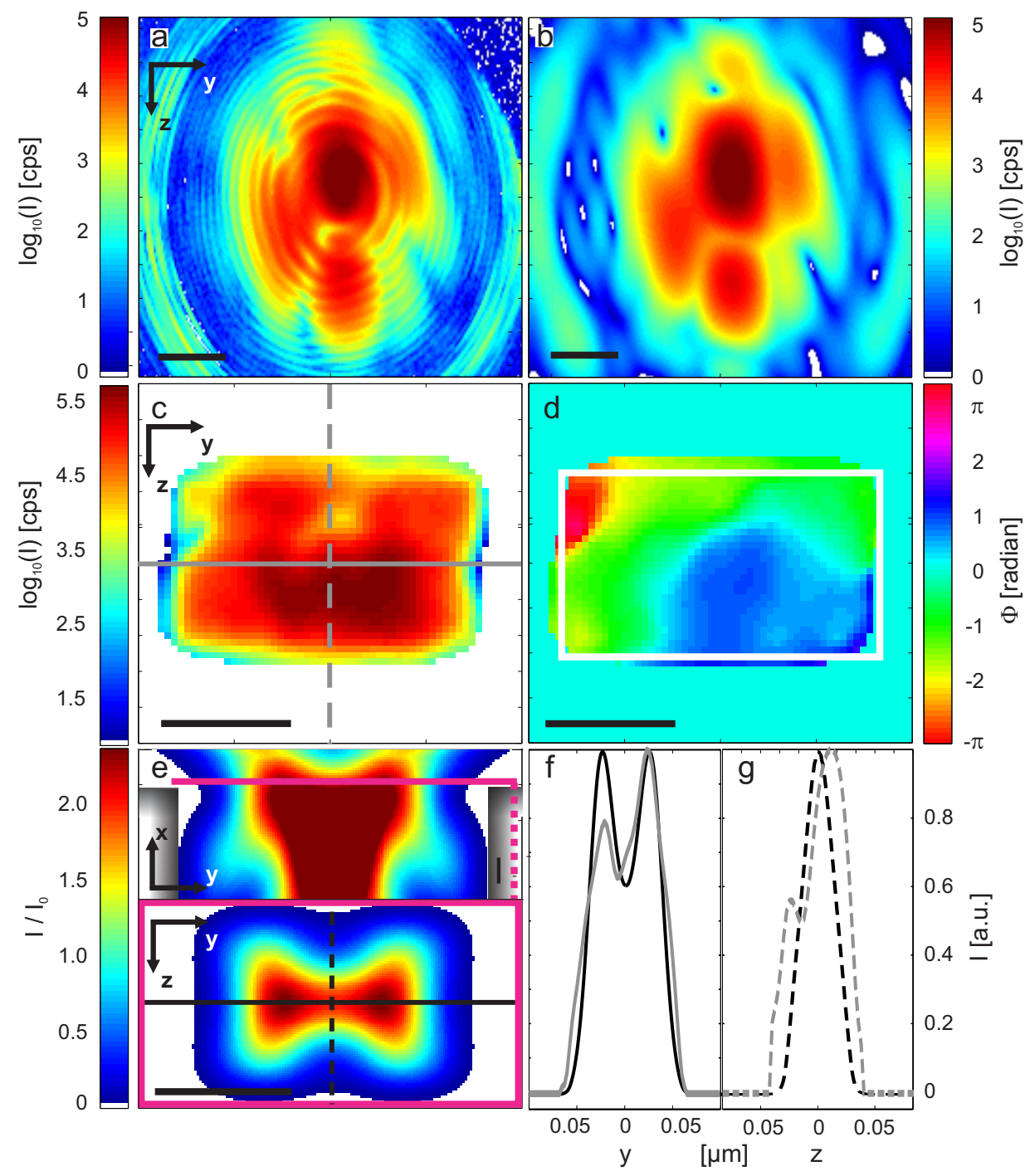

Figure 1.14: Characterization of a $d=113 \mathrm{~nm}$ wide and $\mathrm{h}=80 \mathrm{~nm}$ deep channel. (a) Measurement and (b) reconstruction of the far-field pattern. Scale bars denote $10 \mathrm{~mm}$. (c) Reconstructed amplitude and (d) phase $\Phi$, with a white square marking the initial support. (e) FDSs of the wave field near the channel exit, in side view (top) and as a slice through the exit plane (bottom). All horizontal scale bars denote $50 \mathrm{~nm}$, for (e, top) a vertical scale bar of $10 \mu \mathrm{m}$ is added. $(f)$ Horizontal and $(g)$ vertical intensity profiles from (c) in gray and $(e$, bottom) in black, both normalized to the maximum intensity of the corresponding image. 


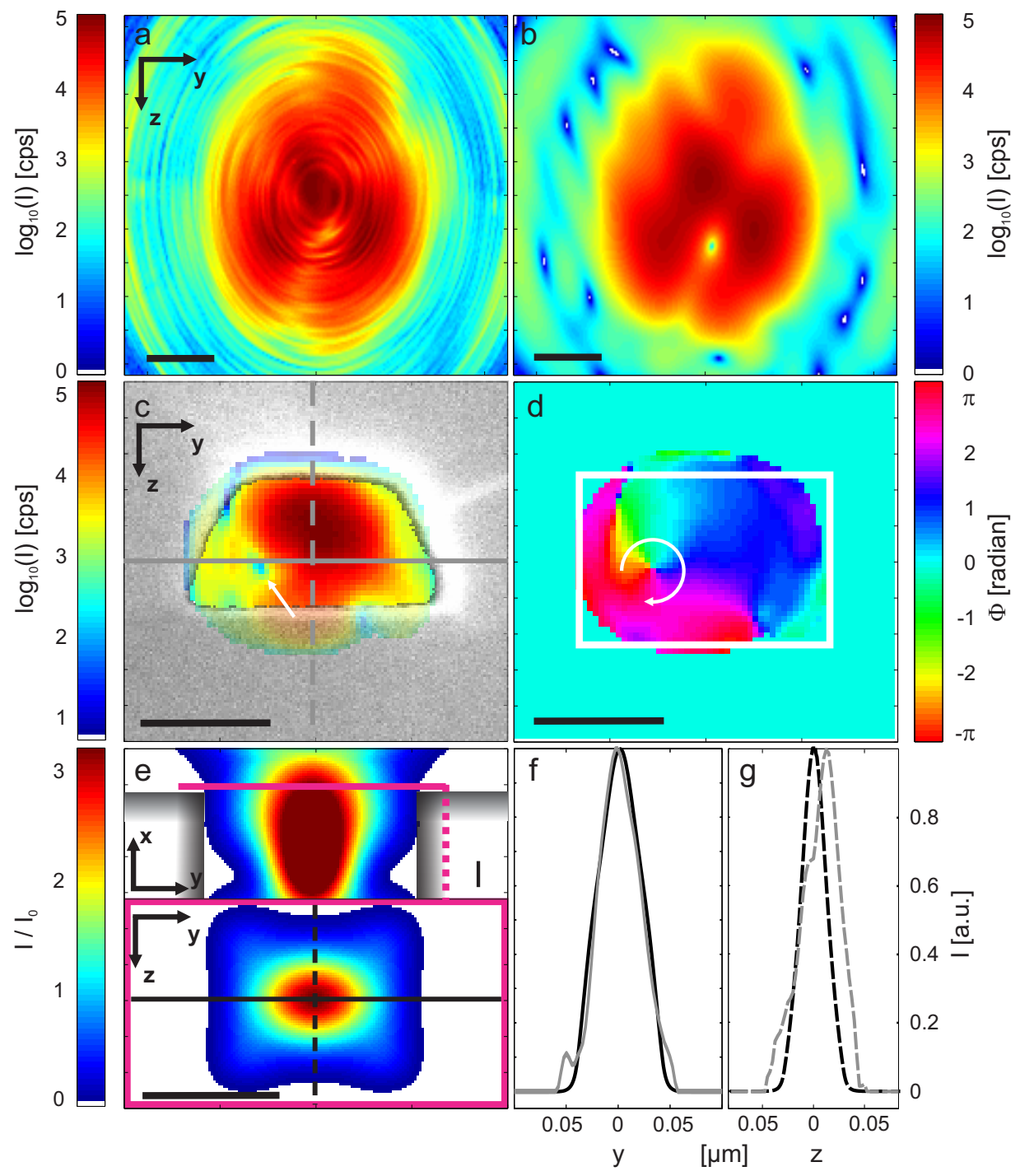

Figure 1.15: Characterization of a $d=82 \mathrm{~nm}$ wide and $h=67 \mathrm{~nm}$ deep channel. (a) Measurement and (b) reconstruction of the far-field pattern. Scale bars denote $10 \mathrm{~mm}$. (c) Reconstructed amplitude with overlaid SEM micrograph and (d) phase $\Phi$, with a white square marking the initial support. (e) FDSs of the wave field near the channel exit, in side view (top) and as a slice through the exit plane (bottom). All horizontal scale bars denote $50 \mathrm{~nm}$, for (e, top) a vertical scale bar of $10 \mu \mathrm{m}$ is added. ( $f$ ) Horizontal and ( $g$ ) vertical intensity profiles from (c) in gray and ( $e$, bottom) in black, both normalized to the maximum intensity of the corresponding image. White arrows in $(c)$ and $(d)$ mark a phase vortex. 


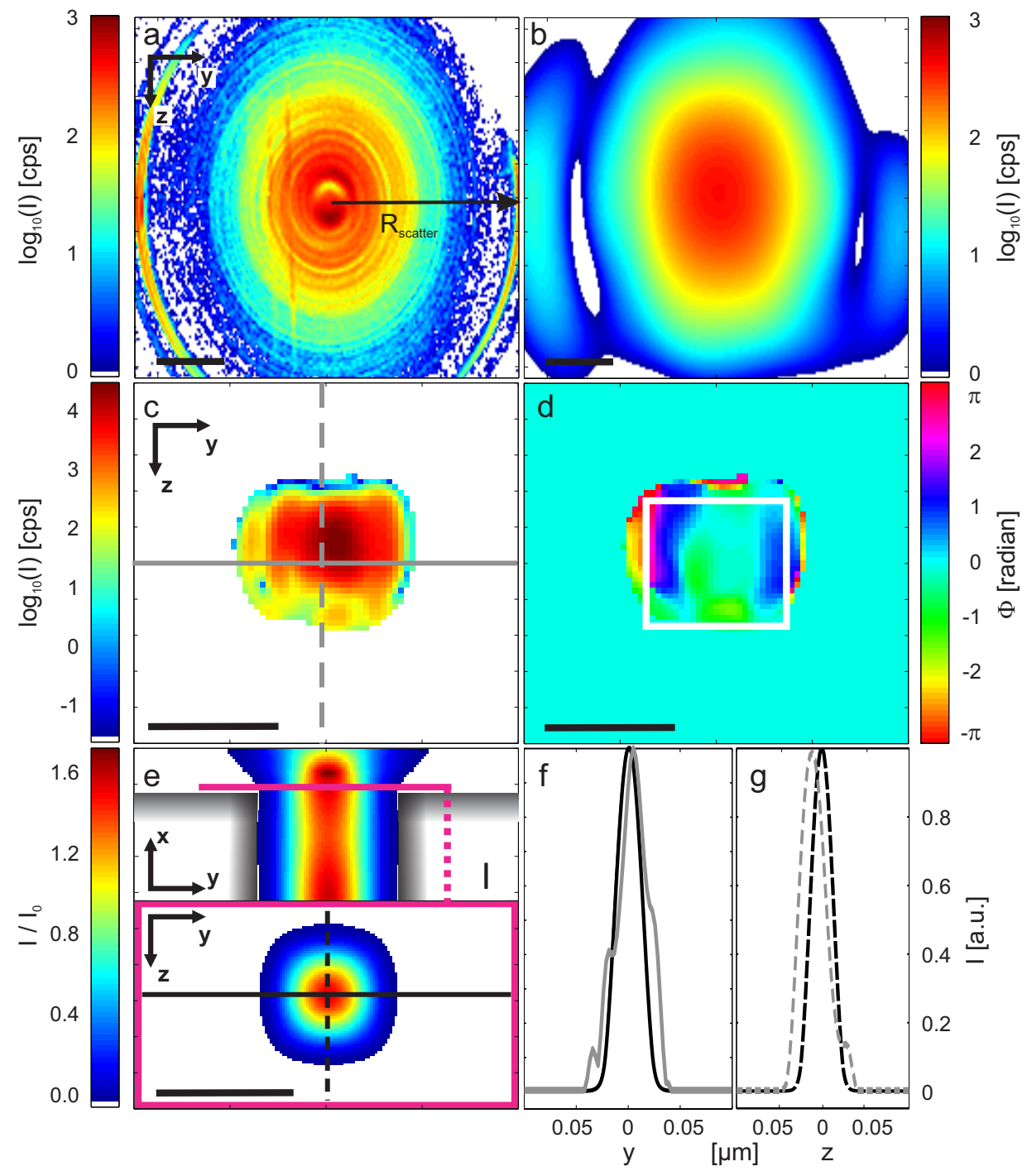

Figure 1.16: Characterization of a $d=43 \mathrm{~nm}$ wide and $\mathrm{h}=45 \mathrm{~nm}$ deep channel. (a) Measurement and (b) reconstruction of the far-field pattern. Scale bars denote $10 \mathrm{~mm}$. (c) Reconstructed amplitude and (d) phase $\Phi$, with a white square marking the initial support. (e) FDSs of the wave field near the channel exit, in side view (top) and as a slice through the exit plane (bottom). All horizontal scale bars denote $50 \mathrm{~nm}$, for (e, top) a vertical scale bar of $10 \mu \mathrm{m}$ is added. ( $f$ ) Horizontal and (g) vertical intensity profiles from (c) in gray and (e, bottom) in black, both normalized to the maximum intensity of the corresponding image. 
At the P10 beamline at PETRA III, DESY, measurements on $L=5.2 \mathrm{~mm}$ long, straight channels [see Fig. 1.14 1.16 (a)] were performed at $E_{p h}=13.8 \mathrm{keV}$. The x-ray beam was focused by a pair of Kirkpatrick-Baez (KB) mirrors to a spot size of $170 \times 390 \mathrm{~nm}$, revealing an intensity of $I_{0}=1.5 \cdot 10^{11} \mathrm{cps}$. The far-fields were recorded by a Pilatus $300 \mathrm{k}$ (Dectris) detector at $5.1 \mathrm{~m}$ behind the waveguide device. The roundish cutouts in the top right corner in the far-field patterns are caused by an aperture in the optical path, which is part of an on-axis light microscope, utilized to pre-align the waveguide device. The intensities of the measured (a) and reconstructed far- and near-fields [(b) and (c), respectively] are given in counts per seconds; all depicted in logarithmic scaling. Additional FDSs (e) were performed and compared to the reconstruction; here the intensities are normalized to the input intensity $I_{0}$ and the sampling is $1 \mathrm{px} / \mathrm{nm}$ in $z$ and $y$-direction and $1 \mathrm{px} / \mu \mathrm{m}$ in $x$-direction.

For all channels, a noticeable maximum intensity of circular shape is observed in the recorded far-fields. The radius of $R_{\text {scatter }} \approx 26 \mathrm{~mm}$ [see Fig. 1.16(a)] corresponds to radiation, deflected at an angle of $0.292^{\circ}$, calculated for a distance of $5.1 \mathrm{~m}$ between waveguide device and detector. As expected from Eq. (1.1), waves impinging on the channel boundaries, parallel to the optical axis $\left(\theta_{1}=0^{\circ}\right)$ are deflected at the critical angle $\theta_{c}=0.226^{\circ}$. Here, this effect is more pronounced than at shorter channels for example with a length of $L=1 \mathrm{~mm}$ (see Fig. 1.5) - since the channel surface is increased, where the radiation is scattered. From the visual impression the reconstructed far-fields (b) are in good agreement with the recorded data (a), especially in view of the shape of the intensity distribution in the central regions of the patterns, which are attributed to the waveguide channel. A minor significance is assigned to the ring-like features, originating from scattering processes (discussed in section 1.2.3. Hence, the percentage deviations are very small: $1.3 \%$ for Fig 1.14 1.2\% for Fig 1.15 and $1.5 \%$ for Fig 1.16 . The corresponding transmission values are $T=0.7 \%, T=0.5 \%$ and $T=0.02 \%$, estimated using the procedure in chapter 4 . Concerning the intensity of far-field pattern, the lack of magnitude is compensated by the homogeneity of the intensity distribution and the flat phase - especially for the smallest channel in Fig. 1.16 - enabling coherent x-ray imaging.

As mentioned above, the complexity of the (mode) structure of the intensity distribution in the far-field patterns decreases with width $d$. This development is even enhanced, since the depth $h$ is also decreased as the channels become thinner (see Fig 1.5. The same tendency is apparent in the reconstructions in Fig 1.14 1.16.(c) and FDSs (e) of the near-fields. For a quantitative analysis, intensity profiles for the reconstructed (gray) and simulated (black) near-fields are compared. These profiles are calculated by summing up all one-dimensional intensity profile lines parallel to the concerned direction, subsequently normalizing them to the maximum of this sum $5^{5}$ For the widest channel in Fig 1.14 the near-field intensity distribution is bow-tie shaped (e, bottom). The intensity profile of the reconstruction (c) matches the simulated profile quite well [see (f)], whereas deviations in this order can easily arise, since micron-sized shifts in $x$-direction cause noticeable changes in the shape of the intensity distribution [see (e, top)]. The horizontal profiles in (f) of the smaller channels (Fig. 1.15 and 1.16)

5 For example, in the case of a horizontal profile, single profile lines for constant values of $y$ are summed up in the whole range of $z$. 
are in good agreement with the simulations as well. Considering the vertical profiles in (g) the reconstructed curves are generally broader and their center is shifted. The enhanced width is attributed to the usage of a support, which is larger than the channel size incorporated in the simulation, accounting for the penetration depth $d_{p}$. The size of the support (white square) relative to the actual channel is shown by an overlaid SEM micrograph in figure $1.15(\mathrm{c})$ and (d). Indeed, areas of the reconstructed intensity even exceed the initial support. There the intensity accounts only a few percent of the maximum value and is attributed to scattering processes inside the channels. The radiation leaking into the cladding near the channel exit is not fully absorbed and contributes to the far-field pattern. Although the support was less restricted in the shrink wrap step, the field in the exit plane is nevertheless of reasonable size underlining the reliability of the reconstruction.

Owing to the invariance of the Fourier transform to lateral shifts, the solution for the far-field remains unchanged when the near-field intensity distribution is shifted in $z$ direction. For this reason, the shifts in (g) can be neglected. The phases vary over the cross-sectional area of the channel, but are almost constant at regions of high intensity [compare (c) and (d)]. As an exception, a phase discontinuity is found in Fig. 1.15. A phase vortex is identified in the lower left corner of the channel (d), marked by an arrow (see also section 6). This is evidenced by the observation of the related minimum in intensity ${ }^{6}$ (c). Next, the field exiting a waveguide grating is reconstructed.
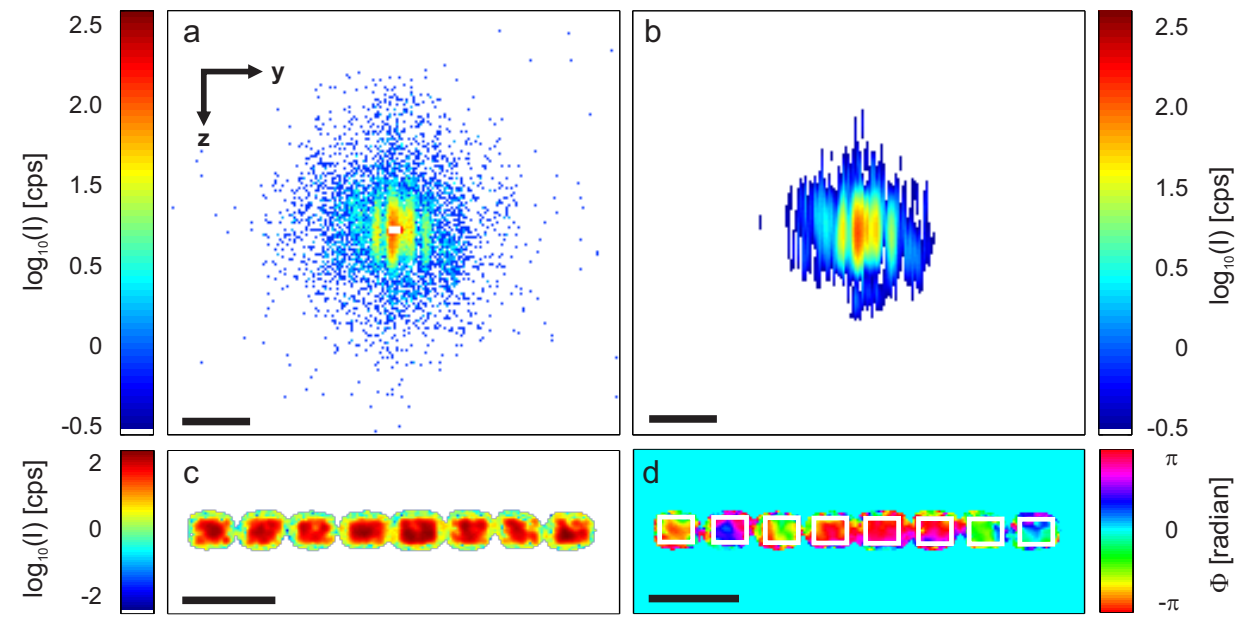

Figure 1.17: Characterization of a waveguide grating. (a) Measurement and (b) reconstruction of the farfield pattern. In (a) the primary beam in the middle of the diffraction pattern is masked out by a white square. Scale bars denote $5 \mathrm{~mm}$. Reconstructed (c) intensity and (d) phase in the exit plane, with a white squares marking the initial support. Scale bars denote $0.5 \mathrm{~mm}$. The intensity does not drop to zero, because the sampling is not sufficient to resolve the point-sized vortex
core. 
The measurement shown in figure 1.17(a) was performed at the BM20 beamline, ESRF at $E_{p h}=11.5 \mathrm{keV}(\lambda=0.107 \mathrm{~nm})$. The far-field was recorded by a Pilatus $100 \mathrm{k}$ (Dectris) detector at a distance of $3.5 \mathrm{~m}$ behind the waveguide device. The x-ray focus revealed an elliptical shape with a size of $120 \mu \mathrm{m} \times 70 \mu \mathrm{m}$, illuminating all channels simultaneously. The signal-to-noise ratio is inferior to the measurements in Fig. 1.14 . 1.16), due to the pronounced over exposure as well as the comparably low intensity of $I_{0}=2 \cdot 10^{11} \mathrm{cps}$, typical for bending magnets which shapes the x-ray beam behind the source. The radiation was filtered by a $\mathrm{Si}(111)$ double crystal monochromator and than focused by a toroidal mirror ( $\mathrm{Si} / \mathrm{Rh}$ ) at a distance $G$ of about $20 \mathrm{~m}$ from the waveguide device [77. The spacial coherence $\xi$ can be calculated by [78]

$$
\xi_{i}=\frac{\lambda \cdot G}{b_{i}} \quad \text { with } \quad i=y, z \text { and } \text { beam size }=b_{y} \cdot b_{z}
$$

For the given parameters, and a beam size of approximately $12.2 \mathrm{~mm} \times 1.2 \mathrm{~mm}$ at the mirror position, the coherence lengths are $\xi_{y}=0.2 \mu \mathrm{m}$ and $\xi_{z}=1.8 \mu \mathrm{m}$. Compared to the structure size of $6 \mu \mathrm{m} \times 0.134 \mu \mathrm{m}$, a coherent illumination is only provided for the central channels.

The measured intensities in the far-field exhibit an interference pattern with vertically oriented fringes, evidencing that the waveguide consists of more than one horizontally arranged channel. Here, an array of 19 channels of $L=2.2 \mathrm{~mm}$ length, each with a size of $201 \mathrm{~nm} \times 134 \mathrm{~nm}$ and a spacing of $S=95 \mathrm{~nm}$ [for definition of $S$ see Fig. 1.7(f)] was tested. The transmission of the grating amounts to $T=21 \%$. Regarding the limited field of view in the object plane, only eight of the nineteen channels are covered by the detector. When the number of the channels in the exit plane is reduced, the interference fringes in the far-field appear less sharp, however, maintaining their spacing. For this reason, using a minor number of channels for the $E R$ algorithm, nevertheless yields to valuable results. The near-field intensity (c) inside the square shaped (red colored) regions is significantly larger than in the surrounding area, exceeding the size of the initial support [white boxes in (d)]. As mentioned above, this is attributed to scattering processes inside the channels, while again the reliability of the reconstruction is evidenced by reasonable lateral dimensions of the reconstructed intensity distribution.

The phase varies continuously over each channel (d), while exhibiting similar values only for the two central channels due to the limited coherence. Furthermore, they reveal neither discontinuities nor high frequency oscillations, thus validating the reconstruction. Its accuracy is also evidenced by the small percentage deviation of $1.7 \%$, calculated by comparing the reconstructed and recorded far-fields. A white square in the center of the measured data masks out the primary beam. This region is not affected by the magnitude constraint, thus allowing the $E R$ algorithm to find an appropriate solution fitting to the measured data. 


\subsection{Characterization of $x$-ray beams}

Besides providing a filtered quasi point-source, waveguides can be used to characterize the incoming beam precisely. Since channels for imaging applications are typically smaller than $100 \mathrm{~nm} \times 100 \mathrm{~nm}$, they facilitate a direct measurement of the larger beam. Lateral scans in $y$-and $z$-direction do not only give an upper limit of the size in the corresponding direction, but rather enable the determination of the intensity distribution within the beam. While the waveguide is scanned across the beam, the transmitted intensity is recorded at every position, revealing a microscopic image of the beam.

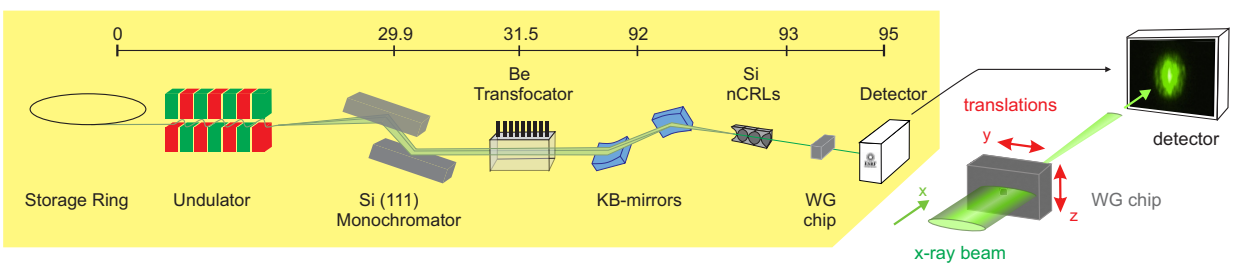

Figure 1.18: Measurement setup at beamline IDI1 at the ESRF. On the left, the path of the $x$-rays is traced back from the waveguide chip to the storage ring, showing optical elements significantly involved in shaping the beam. The right part is an enlarged view on the waveguide chip, illustrating the measuring process. Note that this diagram is not to scale.

In figure 1.18 the measurement setup at beamline ID11 (ESRF) is depicted [79]. The beam $\left(E_{p h}=18 \mathrm{keV}\right)$ exits the storage ring, passes the undulator and is filtered by a $\mathrm{Si}(111)$ double crystal monochromator and then pre-focused by an in-vacuum beryllium transfocator at a distance of about $30 \mathrm{~m} \mathrm{[80]}$. At $92 \mathrm{~m}$ behind the storage ring, a pair of KB-mirrors and 80 Silicon nano-CRLs each with a radius of $75 \mu \mathrm{m}$ confine the beam to its final size. The waveguide chip is positioned in the focal spot of the lenses using motorized stages for aligning in three translational and two rotational degrees of freedom.

At $1 \mathrm{~m}$ behind the waveguide device the far-field pattern is recorded by a Frelon $4 \mathrm{M}$ Detector (ESRF). The data presented in figure 1.19 was recorded using a $21 \mathrm{~mm}$ long waveguide channel with an exit size of $96 \mathrm{~nm} \times 89 \mathrm{~nm}$, which was scanned across the beam. The total intensity at each scan point is depicted in (a), revealing that the beam consist of two sub-beams with a lateral distance of approximately $5 \mu \mathrm{m}$. In the surface plot [inset in (a)] a three dimensional view of the intensity profile of the beam is shown. Gaussian fits to profiles through the maxima of the beams (c-e) yield values of $1.2 \mu \mathrm{m}\left(p_{1}\right), 1.1 \mu \mathrm{m}\left(p_{2}\right)$ and $5.5 \mu \mathrm{m}$ and $5.0 \mu \mathrm{m}\left(p_{3}\right)$ for the full width at half maximum (FWHM), as expectable for the CRLs [81. In (b) a scan in $p_{z}$-direction is depicted, while the $p_{y}$ position was chosen randomly, as it is typical during the early stages in the alignment process. Even though the number of data points is lower, two maxima can clearly be identified, which differ in heights and shapes (FWHM: $3.1 \mu \mathrm{m}$ and $7.8 \mu \mathrm{m}$ ). Since the beam is tilted by $6^{\circ}$ around the optical axis, the relation between this two maxima is sensitive to the exact $p_{y}$ scanning position. Hence, there are $p_{y}$ positions where just a single peak is observed in the scan. Assuming this peak to be the absolute maximum, the second peak may be overlooked. 

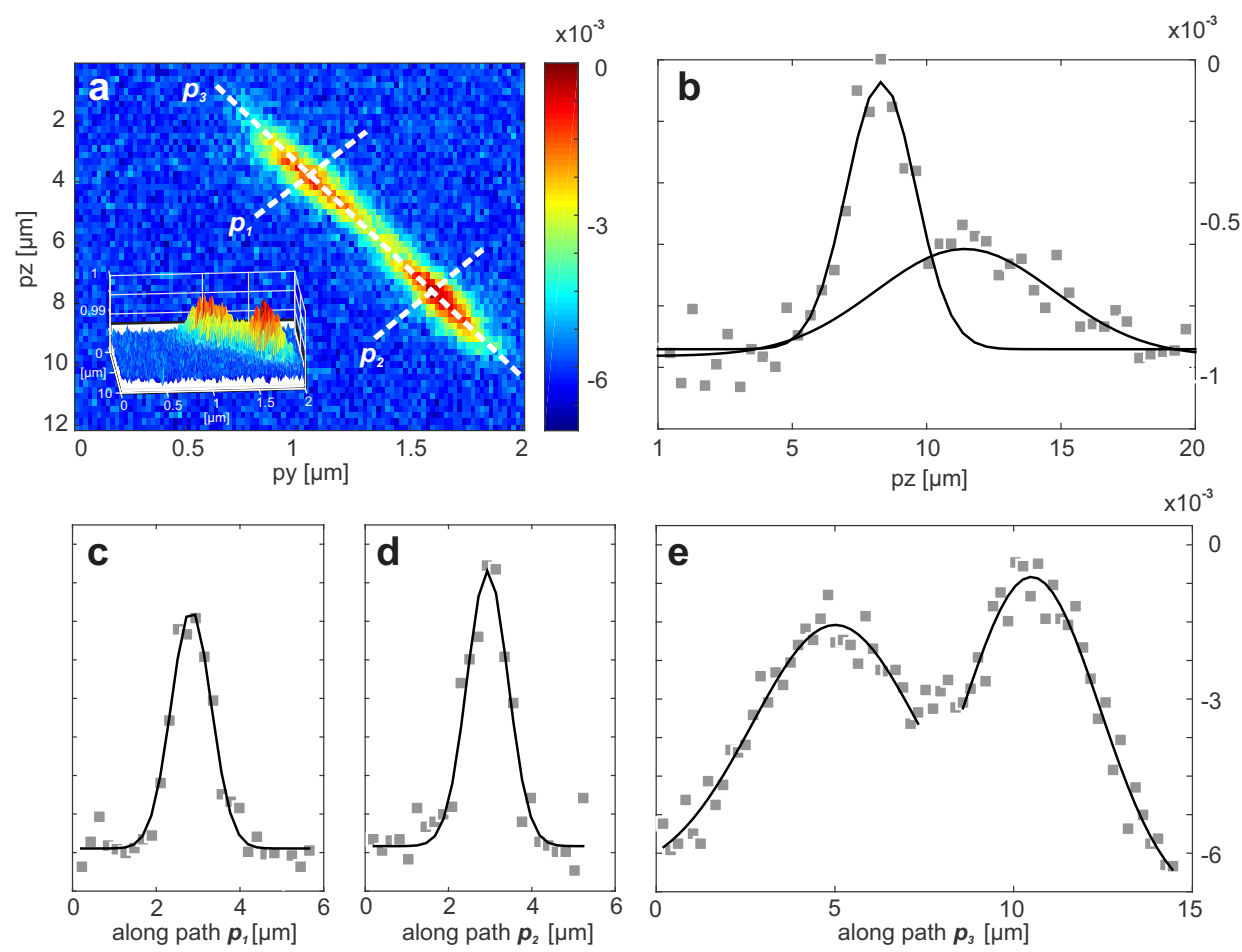

Figure 1.19: Determining the beam properties with waveguides. (a) Overview scan of the beam with $58 \times 100$ images (horiz. $\times$ vert.) and a surface plot of the same data set in the lower left corner. Note the different axis sizing. (b) Intensity profile along the $p_{z}$-direction crossing the beam at a random $p_{y}$-position. (c)-(e) Intensity profiles (gray dots) extracted from (a) through the axes of symmetry along the dashed lines according to the letters and the corresponding Gaussian fits (black line). For all scans a counting time of 1 s was chosen. All intensities are normalized to the maximum intensity and plotted in logarithmic scaling.

In combination with the scanning method depicted in (a), waveguides can be utilized to get direct access to the shape and the intensity distribution of the beam, helping to identify the spot in the incoming field which is most suitable for the measurements. The photon guiding channels enable for monitoring the intensity distribution of the beam while the optical elements for manipulating or focusing are adjusted. This makes waveguides a powerful tool for a precise beamline alignment procedure. 



\section{Chapter 2}

\section{High aspect ratio $x$-ray wave- guide channels fabricated by e-beam lithography and wafer bonding}

Reprinted from

H. Neubauer, S. Hoffmann, M. Kanbach, J. Haber, S. Kalbfleisch, S. Krüger, and T. Salditt High aspect ratio $x$-ray waveguide channels fabricated by e-beam lithography and wafer bonding Journal of Applied Physics, 115(21):214305 (2014)

with the permission of AIP Publishing. 


\title{
High aspect ratio $x$-ray waveguide channels fabricated by e-beam lithography and wafer bonding
}

\author{
H. Neubauer, S. Hoffmann, M. Kanbach, J. Haber, S. Kalbfleisch, S. P. Krüger, \\ and T. Salditt ${ }^{\mathrm{a})}$ \\ Institut für Röntgenphysik, Universität Göttingen, Friedrich-Hund-Platz 1, 37077 Göttingen, Germany
}

(Received 14 April 2014; accepted 17 May 2014; published online 3 June 2014)

\begin{abstract}
We report on the fabrication and characterization of hard $\mathrm{x}$-ray waveguide channels manufactured by e-beam lithography, reactive ion etching and wafer bonding. The guiding layer consists of air or vacuum and the cladding material of silicon, which is favorable in view of minimizing absorption losses. The specifications for waveguide channels which have to be met in the hard $\mathrm{x}$-ray range to achieve a suitable beam confinement in two orthogonal directions are extremely demanding. First, high aspect ratios up to $10^{6}$ have to be achieved between lateral structure size and length of the guides. Second, the channels have to be deeply embedded in material to warrant the guiding of the desired modes while absorbing all other (radiative) modes in the cladding material. We give a detailed report on device fabrication with the respective protocols and parameter optimization, the inspection and the optical characterization. (C) 2014 Author(s). All article content, except where otherwise noted, is licensed under a Creative Commons Attribution 3.0 Unported License. [http://dx.doi.org/10.1063/1.4881495]
\end{abstract}

\section{INTRODUCTION}

Hard $\mathrm{x}$-ray nanobeams with cross sections in the range of $d \simeq 10-100 \mathrm{~nm}$ enable novel nanoscale analytic techniques, adding nanoscale real space resolution to x-ray spectroscopy and diffraction, and enabling novel variants of coherent imaging and holography. A variety of optical elements can be used to generate x-ray nanobeams, such as Fresnel zone plates, ${ }^{1-4}$ multilayer Laue lenses, ${ }^{5}$ multilayer zone plates, ${ }^{6}$ compound refractive lenses, ${ }^{7-9}$ curved mirrors (e.g., in Kirkpatrick-Baez (KB) geometry ${ }^{10,11}$ ), or $\mathrm{x}$-ray waveguides, ${ }^{12-17}$ as well as combinations of these elements. ${ }^{6,18,19}$ The different optical elements impose significant challenges for nanostructuring and metrology, and progress in this field is often limited by the corresponding bottlenecks in fabrication. This is particularly the case for x-ray waveguides (WG), which deliver coherence filtered $\mathrm{X}$-ray radiation with nanoscale dimensions in one spatial $(1 \mathrm{DWG})^{12-17,20,21}$ or two spatial dimensions (2DWG). ${ }^{22-24}$

Depending on the materials employed for the guiding and cladding layers, waveguides are capable to deliver beams of about $10 \mathrm{~nm}$ in cross section, ${ }^{25}$ comparable to the record values achieved by other types of focusing optics. ${ }^{6,26}$ By tailoring the coherence properties and cross section of $\mathrm{x}$-ray beams, waveguides can extend the function of macroscopic slits and pinholes of conventional x-ray experiments to x-ray nanobeam optics. In particular, in the monomodal regime they also enable the control of the wavefront phase highly desirable for coherent $\mathrm{x}$-ray imaging and holography. ${ }^{24,27-29}$ To block the multi-keV photon energy beam in the cladding material, rather long channels are needed with aspect ratios in the range of $10^{4}-10^{6}$, depending on the photon energy $E$ and cross section $d$.

\footnotetext{
a)Electronic mail: tsaldit@gwdg.de
}

$\mathrm{X}$-ray propagation in waveguide channels has been studied both analytically ${ }^{30-32}$ and by finite difference (FD) simulations, ${ }^{17,32-34}$ including generalizations to more complex structures and effects including both thickness variations and roughness, ${ }^{35,36}$ which illustrate the challenges associated with fabrication.

In particular, the extreme aspect ratios required for $\mathrm{x}$-ray waveguides impose tremendous challenges in fabrication. Note that one-dimensional beam confinement can be easily achieved by planar waveguides (1DWG) fabricated by thin film deposition, ${ }^{37}$ but is only of limited use. The vast majority of applications, in particular holographic imaging, requires two-dimensional waveguides (2DWG), as first demonstrated by Pfeiffer et al., ${ }^{22}$ however, with impractically low efficiencies, or at the expense of an inherent astigmatism in the case of planar waveguides in crossed geometry. ${ }^{37}$ When replacing the coupling geometry and combining the waveguides with high gain pre-focusing $\mathrm{KB}$ mirrors, the waveguide exit flux was increased to $I_{w g} \simeq O\left(10^{6}\right)$ photons per second, ${ }^{23,24}$ which is still too low for most imaging applications, e.g., holography experiments. In earlier approaches, the waveguide channels consisted of polymer stripes defined by e-beam lithography and coated with a silicon cladding. ${ }^{23,24}$ Ideally, however, waveguide channels should be empty (air, vacuum), since a solid core material leads to unnecessary absorption, unwanted phase front modulations based on density variations, and in the case of polymer channels also drifts resulting from beam damage in the core material. To overcome these problems, a novel fabrication scheme based on dry etching of channels into silicon wafers has been reported previously, ${ }^{38,39}$ using an etch mask defined by e-beam lithography, removal of the mask and subsequent capping by a second wafer bonded on top of the first. However, only $\mu$ m-sized cross sections were achieved in both directions. Only recently, this fabrication approach was optimized and refined to achieve control over both interface 
quality and channel dimensions in the relevant sub- $100 \mathrm{~nm}$ range. In fact, first $\mathrm{x}$-ray imaging results obtained with such a channel have been presented, ${ }^{18}$ without giving the details and refinements of the fabrication process, which is the scope of the present paper. Here, we give the first report on the fabrication of wafer-bonded 2DWG channels in the relevant sub- $100 \mathrm{~nm}$ range, addressing the detailed steps and protocols as well as including the most recent optimizations which result in a waveguide exit flux of $I_{w g} \simeq O\left(10^{8}\right) \mathrm{ph} / \mathrm{s}$, as measured at the P10 beamline of the PETRA III storage ring of DESY for a recently fabricated waveguide channel presented here.

\section{EXPERIMENTAL METHODS: FABRICATION}

Fig. 1(a) shows a schematic representation of the waveguide fabrication steps. An electron beam resist spin-coated on a silicon wafer is structured by electron beam lithography, providing an etching mask. The structure is transferred into the substrate by reactive ion etching (RIE). Subsequently, the mask is removed and a second wafer is attached by hydrophilic wafer bonding ${ }^{40}$ under cleanroom conditions. As a substrate, one-sided polished $1000 \pm 25 \mu \mathrm{m}$ thick 4 in. (100) Si-Wafers (silchem) have been used. The fabrication steps have been carried out at varied parameters, as presented in Table I. In the following, we describe the process based on the parameters of protocol I, given as an example. We will refer to the parameters of the other protocols where appropriate. The poly-methyl-methacrylate (PMMA) e-beam resist "A2" (MicroChem) was spincoated onto the substrate at 2000 revolutions per minute $(\mathrm{rpm})$, providing a $100 \mathrm{~nm}$ layer of positive e-beam resist. The resist was exposed with the lithographic pattern of parallel channels, as depicted in Fig. 4(b). The exposure was performed by a Raith e_LiNE lithography system using the Fixed Beam Moving Stage (FBMS) exposure mode, moving an interferometric laser stage below a stationary electron beam, thus avoiding the necessity of write-field stitching. Note that only this
TABLE I. Fabrication parameters, according to the different protocols (I-IV) evolving from iterations in fabrication and characterization: spincoating velocity, etching gas composition and etching time, cleaning procedure before bonding, annealing temperature, and time. All other parameters were kept constant. In addition, the channel depth evaluated after dicing from SEM micrographs is shown.

\begin{tabular}{lccccc}
\hline \hline & $\begin{array}{c}\text { Coating } \\
(\mathrm{rpm})\end{array}$ & $\begin{array}{c}\mathrm{SF}_{6}: \mathrm{CHF}_{3} \\
(\mathrm{sccm})\end{array}$ & Cleaning & Bonding & $\begin{array}{c}\text { Channel } \\
\text { depth (nm) }\end{array}$ \\
\hline I & 2000 & $14: 35(30 \mathrm{~s})$ & $\mathrm{O}_{2}(30 \mathrm{~min})$ & $1050^{\circ} \mathrm{C}(4 \mathrm{~h})$ & 24 \\
II & 2000 & $10: 40(40 \mathrm{~s})$ & RCA-SC1 & $1050^{\circ} \mathrm{C}(4 \mathrm{~h})$ & 30 \\
III & 2000 & $14: 35(40 \mathrm{~s})$ & RCA-SC1 & $1050^{\circ} \mathrm{C}(4 \mathrm{~h})$ & 50 \\
IV & 1000 & $14: 35(50 \mathrm{~s})$ & RCA-SC1 & $750^{\circ} \mathrm{C}(2 \mathrm{~h})$ & 70 \\
\hline \hline
\end{tabular}

approach enables for the production of high aspect ratio waveguide channels in the first place, as the mismatch from write-field stitching typically exceeds the waveguide channel dimension. The structures were developed in a 1:3 mixture of methylisobutylketone (MIBK): isopropanol (IPA), with a typical volume of $80 \mathrm{ml}$, to which $1.6 \mathrm{ml}$ of distilled water was added. The samples were kept in the developer for $30 \mathrm{~s}$ at a temperature of $10^{\circ} \mathrm{C}$, followed by $30 \mathrm{~s}$ in IPA at room temperature, serving as a stopper. ${ }^{41}$ Subsequently, the sample was rinsed in a nitrogen gas flow. The structure was transferred into the substrate by reactive ion etching (Plasmalab System 100, Oxford Instruments Plasma Technologies), applying a $40 \mathrm{sccm} \mathrm{CHF}_{3}$ and $10 \mathrm{sccm} \mathrm{SF}_{6}$ gas flow at a power of $100 \mathrm{~W}$ for $48 \mathrm{~s}$ at room temperature, or a variation of this recipe. Alternatively, a mixture of $\mathrm{SF}_{6}$ and $\mathrm{CF}_{4}$ can be used, yielding results of comparable quality both in etching depth and profile. Part of the wafer area was covered by an additional aluminum foil mask in order to increase the etching rate and to assure uniformity, yielding etching depths of $30-80 \mathrm{~nm}$, depending on the protocol. The subsequent mask removal involved a 20 min acetone bath at $50{ }^{\circ} \mathrm{C}$, a distilled water, IPA and dry nitrogen rinse, followed by a $20 \mathrm{~min}$ IPA bath at $50^{\circ} \mathrm{C}$ and a final nitrogen rinse. In (a)
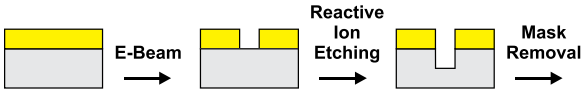

(b)
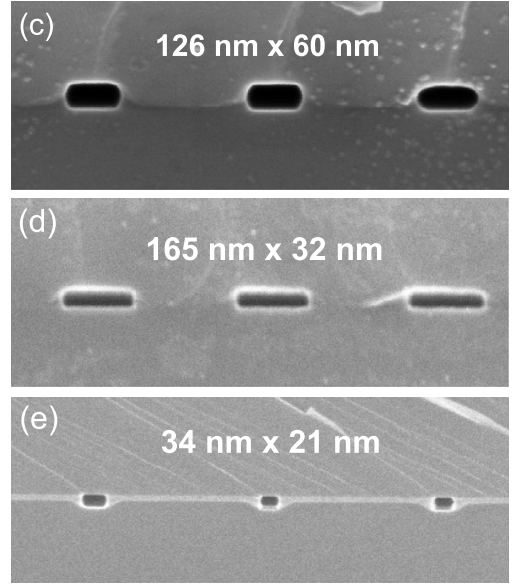

FIG. 1. (a) Sketch of waveguide processing sequence: resist deposition, e-beam exposure, reactive ion etching, mask removal, and finally wafer bonding. (b) Schematic of air-filled channel capped by a top wafer bonded to the substrate. ((c)-(e)) SEM micrographs of waveguide channel entrances, with (d) comparable to the waveguide used for Fig. 5. (f) Photograph of waveguide chip as cut by the wafer dicing machine. 
order to ensure a clean wafer surface, this step was followed in protocol I by a $30 \mathrm{~min}$ RIE plasma cleaning, using 50 $\mathrm{sccm} \mathrm{O}_{2}$ at a power of $300 \mathrm{~W}$ at room temperature, which also served as a hydrophilization of the wafer surface in order to enhance bonding strength.

Since the $\mathrm{O}_{2}$ plasma treatment was found to affect the channel shape function and interface quality during the high temperature annealing step of wafer bonding, plasma cleaning time was therefore reduced or omitted in other protocols. Note that plasma exposure can introduce defects and dislocations to the silicon oxide layer after a few seconds. ${ }^{42}$ Instead, a dip in a DI water bath for a few minutes and a $15 \mathrm{~min}$ RCA-SC1 clean ${ }^{43}$ at $65^{\circ} \mathrm{C}$ can be used to ensure surface cleanliness. Furthermore, to avoid the formation of voids between the two bonded wafers originating from the thermal decomposition of surface contaminants or the desorption of hydrogen, ${ }^{42}$ a reduced annealing temperature ${ }^{44}$ was chosen in protocol IV.

In order to convert the etched surface pattern into buried channel structures, a second, clean $\mathrm{Si}$ wafer of the same batch was attached to the structured wafer manually, promoting a van-der-Waals type pre-bonding, followed by formation of covalent bonds in a thermal furnace (L9/13/P320, Nabertherm) under air atmosphere. ${ }^{40}$ The sample was heated at a rate of $3^{\circ} \mathrm{C} / \mathrm{min}$ from room temperature up to $1050^{\circ} \mathrm{C}$. Temperature was kept constant at $1050{ }^{\circ} \mathrm{C}$ for $4 \mathrm{~h}$, and the sample was cooled down to room temperature inside the furnace over a period of $10 \mathrm{~h}$. During the thermal treatment, the sample was positioned on three ceramic distance pieces, ensuring optimum heat flow. In a wafer dicing machine (DAD 321, DISCO), the samples were finally cut to a width of $5 \mathrm{~mm}$ and a length $l$, depending on the requirements of the experiment, in particular, the photon energy. For the results reported here, lengths of $l=1.0 \mathrm{~mm}(7.9 \mathrm{keV}), l=5.24 \mathrm{~mm}$ $(13.8 \mathrm{keV})$ and $l=13 \mathrm{~mm}(17.5 \mathrm{keV})$, were chosen for the respective experiments. The blade (NBC-ZB 1070, $150 \mu \mathrm{m}$ thickness, $5 \mu \mathrm{m}$ grain size, DISCO) was operated at a feed rate of $0.5 \mathrm{~mm} / \mathrm{s}$ and a positioning precision of $10 \mu \mathrm{m}$. As a direct cut through the sample results in material smearing and also often clogging of the channel openings resulting from blade roughness, the sample was cut from both sides, leaving a section of $100 \mu \mathrm{m}$ thickness connecting the two pieces. By applying a slight pressure, the connection can be broken, yielding sufficiently clean and open waveguide entrances. Optionally, a focused ion beam (FIB) source (Nova Nano Lab 600, FEI) was used to further clean the $17.5 \mathrm{keV}$ samples on both waveguide entrance and exit by successive silicon ablation. Thus, any deviation from the ideal waveguide shape, which may have occurred due to deformations during the breaking, can be corrected.

Structures have been investigated in view of changes in structure width and roughness during the fabrication process. Fig. 2 shows SEM micrographs for the processing steps of exposure and development, etching and mask removal, as well as thermal treatment for one and the same structure. Note that this last step was performed without a capping wafer for inspection purposes, as enclosed channels will not be easily accessible by SEM anymore. Interestingly, during the annealing process a strong decrease in both channel width

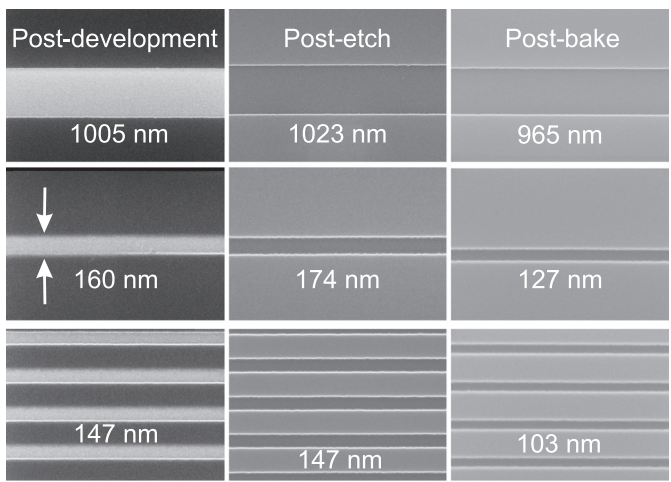

FIG. 2. Evolution of structure width and roughness during fabrication, for different initial channel widths. SEM micrographs, from left to right: Resist after development, etched structure after mask removal and structure after thermal treatment (without capping wafer). Both structure width and sidewall roughness are decreased significantly during heating.

and roughness is observed. The smaller the original structure, the more pronounced this effect becomes. We attribute this effect to a diffusion-driven rearrangement of surface atoms, fed by the energy provided by thermal treatment. As the system tends to minimize its free surface energy, channel wall roughness will be reduced, hence structures improve in quality. Consequently, the reduction in channel width has to be taken into account when choosing an appropriate pattern for exposure, as narrow channels might close while thermally treated. If, in spite of this effect, a further reduction of line width is required, the temperature of the developer can be reduced. ${ }^{41}$ Thus, the development of partially exposed areas at the edge of the structure is prevented, and the resulting pattern will be as precise as defined by means of electron lithography.

Additionally, after performing the pre-bond processing step of attaching the two wafer surfaces to each other, an infrared camera was used for sample inspection, see Fig. 3(a). As even the enclosure of a $1 \mu \mathrm{m}$-sized dust particle may result in a mm-sized air bubble preventing a successful bonding, ${ }^{40}$ samples were inspected using an InGaAs-near-infrared camera (XS-Base, Xenics) at different magnifications. If an air bubble is detected, it can be removed manually by applying an external pressure to the sample, see Figs. 3(b) and 3(c), or the pre-bonded sample can be reopened with the help of a razor blade in order to exchange the capping wafer. Figs. 3(d) and 3(e) shows embedded structures after the annealing step, localizing $500 \mathrm{~nm}$ sized features. Thus, the correct position of the embedded structures after dicing the sample can be verified easily.

\section{EXPERIMENTAL CHARACTERIZATION}

Waveguide optical characterization was performed at the undulator beamline ID22Ni of ESRF, Grenoble, and at the GINIX (Göttingen Instrument for Nano-Imaging with X-rays) instrument of beamline P10 of DESY, Hamburg. ${ }^{45,46}$ Fig. 4(a) shows a schematic of the experimental setup, which in both cases used a $\mathrm{KB}$ mirror system to focus the beam 


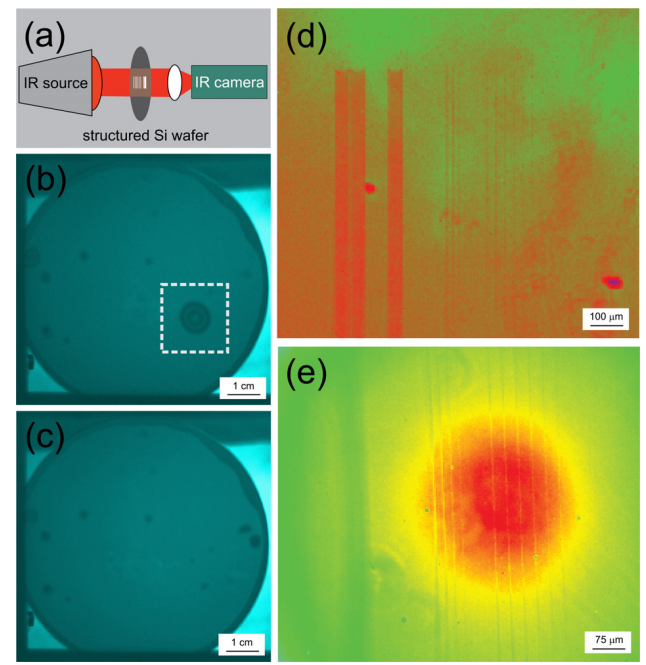

FIG. 3. (a) Inspection of structures embedded in bonded silicon wafers by infrared light in transmission geometry. (b) Wafer with enclosed air bubbles (highlighted by dashed line). (c) Wafer after defined removal of one specific air bubble. (d) Embedded structures in $20 \times$ magnification. (e) Embedded structures in $40 \times$ magnification (smallest visible structure $500 \mathrm{~nm}$ in width).

onto the waveguide entrance. At ID22Ni the focal spot size of the KB was $D_{\mathrm{h}}=129 \mathrm{~nm}$ (FWHM) and $D_{\mathrm{v}}=166 \mathrm{~nm}$ (FWHM), for the horizontal and vertical directions, respectively. The waveguide length $l=13 \mathrm{~mm}$ was optimized to the photon energy $E=17.5 \mathrm{keV}$, assuring sufficient absorption of the radiative modes.

At the GINIX endstation, two different settings were used for two beamtimes: (beamtime GINIX-1) $E=7.9 \mathrm{keV}$ and $D_{\mathrm{h} / \mathrm{v}}=530 / 520 \mathrm{~nm}$ (FWHM), and (beamtime GINIX-2) $E=13.8 \mathrm{keV}$ and focal spot size $D_{\mathrm{h} / \mathrm{v}}=370 / 170 \mathrm{~nm}$ (FWHM). For beamtime GINIX-1, the same waveguide as for the beamtime at ID2 $2 \mathrm{Ni}$ was used, but diced to a shorter length $l=1 \mathrm{~mm}$ to account for the smaller energy. In order to simplify the waveguide alignment in the beam, the lithographic pattern was designed containing a number of parallel waveguide channels with a lateral periodicity of $30 \mu \mathrm{m}$, including a variation of the individual channel width (see Fig. 4(b)), as well as additional areas which act as one-dimensional planar waveguides (1DWG), since the corresponding channel width, i.e., $50 \mu \mathrm{m}$ and $100 \mu \mathrm{m}$, respectively, was much larger than the beam, requiring only the fine alignment of one translation and one rotational degree of freedom.

Fig. 5 shows the measured farfield intensity distribution with corresponding simulations (GINIX-1 and ID22Ni). The settings and parameters for the presented farfield pattern were the following: (a) photon energy $7.9 \mathrm{keV}$, channel length $1 \mathrm{~mm}$, pre-focusing with KB mirrors, direct illumination CCD detector (LCX, Princeton), exposure time $100 \mathrm{~s}$ (GINIX-1 setting), (d) photon energy $17.5 \mathrm{keV}$, channel length $13 \mathrm{~mm}$, pre-focusing with $\mathrm{KB}$ mirrors, pixel detector (Maxipix, ESRF), exposure time $2 \mathrm{~s}$ (ID22Ni setting).

In (b) the modulus of the autocorrelation function obtained by inverse Fourier transform of the data shown in (a) is plotted. Gaussian fits to the peak center along the two principle axes yield a width (FWHM) of $69 \mathrm{~nm}$ (horizontal)
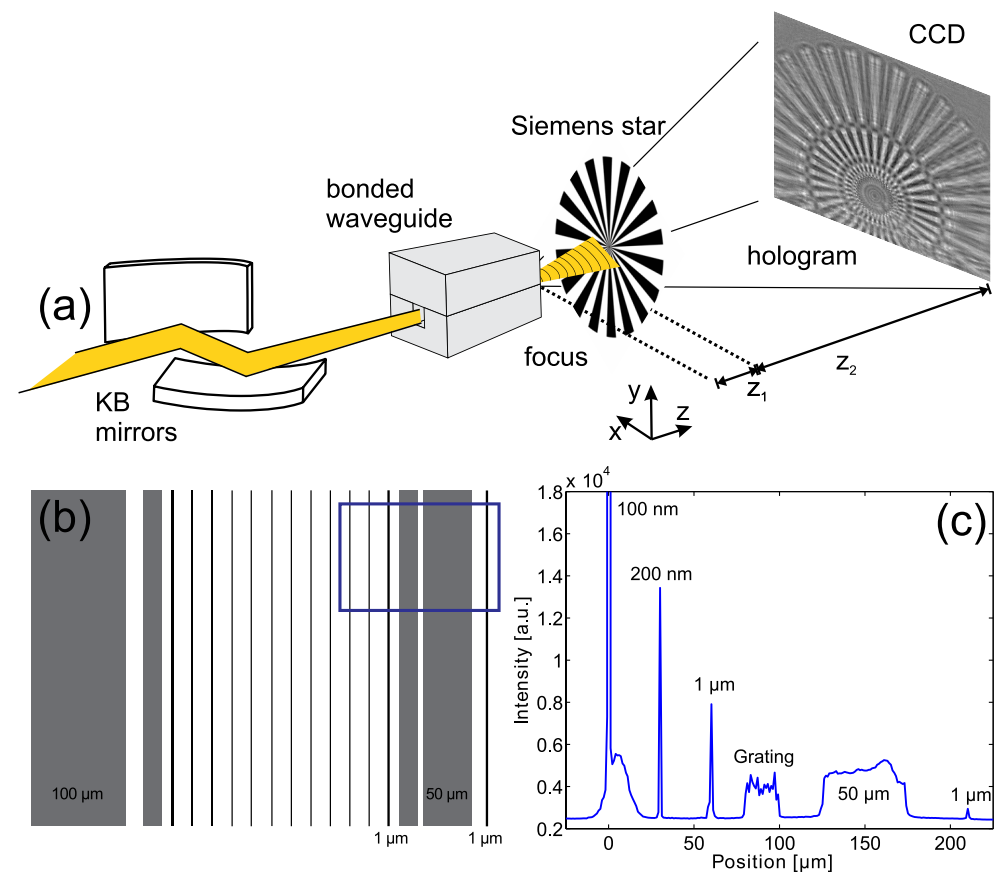

FIG. 4. (a) Holographic imaging setup as used in ESRF (ID22Ni) and PETRA III (GINIX, P10) experiments to determine the waveguide imaging resolution using a test structure. ${ }^{18}$ (b) Typical layout, with several channels on the chip enabling higher throughput in the characterization of optical properties. In addition to the channels, larger structures are placed at the sides, facilitating the alignment of rotational and translational degrees of freedom. (c) Lateral (horizontal) scan helping to identify the individual channel positions as marked in (b), with rotations and vertical position optimized to the $100 \mathrm{~nm}$ channel. 

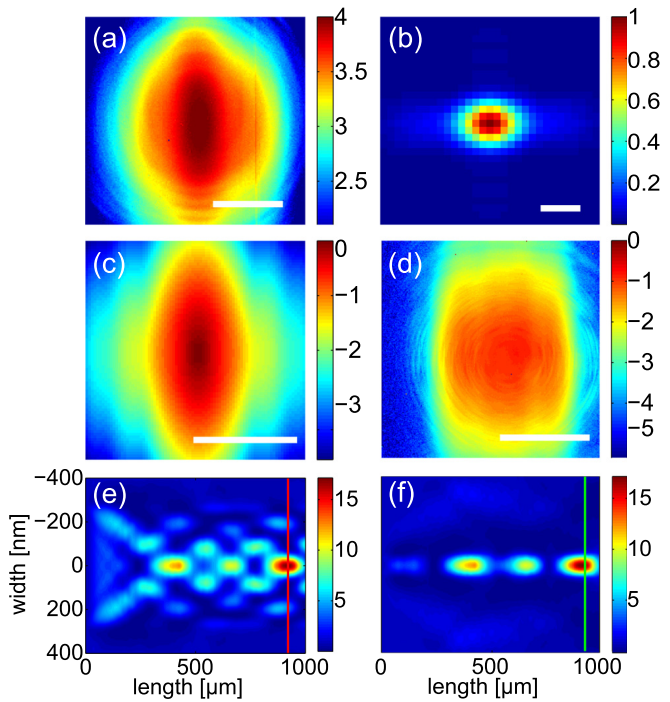

FIG. 5. Comparison of ((a), (b), (d)) experimental results with ((c), (e), (f)) numerical simulations for a $140 \mathrm{~nm} \times 24 \mathrm{~nm}$ waveguide channel. (a) Farfield intensity distribution with logarithmically coded photon counts of a $100 \mathrm{~s}$ exposure recorded at $7.9 \mathrm{keV}$ photon energy and waveguide channel length $l=1 \mathrm{~mm}$ (GINIX -1 experimental settings). Scale bar: $0.1 \mathrm{deg}$. (b) Modulus of the autocorrelation function corresponding to the data shown in (a). Scale bar: $50 \mathrm{~nm}$. (d) Farfield intensity distribution with logarithmically normalized intensity, as recorded over $2 \mathrm{~s}$ at $17.5 \mathrm{keV}$ photon energy and waveguide channel length $l=13 \mathrm{~mm}$ (ID22Ni experimental settings). Scale bar: 0.1 deg. ((c), (e), (f)) Simulation results (finite difference calculations ${ }^{33}$ ) for $E=17.5 \mathrm{keV}$ and $l=13 \mathrm{~mm}$, corresponding to the experimental settings of ID22Ni. (c) The simulated farfield pattern obtained by the squared modulus of the Fourier transform of the calculated exit wave (nearfield). ((e), (f)) The simulated nearfield intensity distribution in the (e) $x z$ and (f) $y z$ plane of the channel, corresponding to the $140 \mathrm{~nm}$ and $24 \mathrm{~nm}$ wide directions, shown for the entrance section $(0 \leq z \leq 1 \mathrm{~mm})$ of the waveguide channel.

and $44.5 \mathrm{~nm}$ (vertical). The finite difference simulation ${ }^{33}$ of the nearfield and corresponding farfield distribution, as obtained by the modulus squared of the Fourier transform of the nearfield, are shown in (c), corresponding to the parameters of the experiment shown in (d). In (e,f), the intensity distribution inside the waveguide channel (d) in the entrance region (first $\mathrm{mm}$ ) is shown, namely by centered cuts in (e) the horizontal $x z$ and (f) the vertical $y z$ planes. Note that the multimodal propagation of the guided wave results in a periodic pattern significantly smaller than the channel width. Gaussian fits along the principal axes (see red and green lines) yield $18.2 \mathrm{~nm}$ and $17.2 \mathrm{~nm}$ for $x z$ and $y z$, respectively. Multimodal propagation leads to a beating effect, i.e., periodic field patterns due to the superposition of the excited modes. In the horizontal direction with $140 \mathrm{~nm}$ channel width the number of modes is higher than in the vertical direction where the beam is confined by a channel height of $24 \mathrm{~nm}$ (see also Fig. 6). After about $900 \mu \mathrm{m}$, positive interference is particularly high for both planes simultaneously, yielding a plane of minimum beam width, as indicated by the red and green lines. This shows that beam confinement can be much smaller than the channel cross section in certain planes.
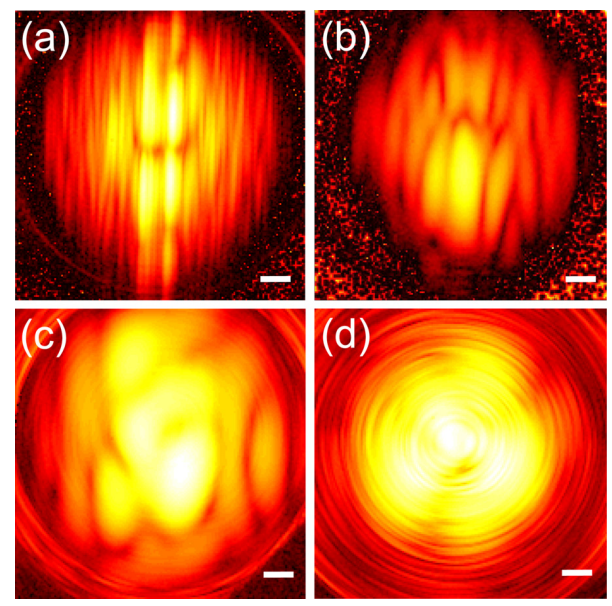

FIG. 6. Farfield intensities depending on waveguide width, using a waveguide chip of a length $l=5.24 \mathrm{~mm}$. The beamline was operated at $13.8 \mathrm{keV}$. The intensity (logarithmic plot) was recorded within $1 \mathrm{~s}$ and is normalized to the maximum value of the intensity distribution shown in (d). Detector: Pilatus $300 \mathrm{~K}$, ROI: $170 \mathrm{px} \times 170 \mathrm{px}$. Channel dimensions: (a) $982 \mathrm{~nm} \times 82 \mathrm{~nm}$, (b) $317 \mathrm{~nm} \times 79 \mathrm{~nm}$, (c) $153 \mathrm{~nm} \times 73 \mathrm{~nm}$, and (d) $82 \mathrm{~nm} \times 67 \mathrm{~nm}$, scalebars: $3 \mathrm{~mm}$.

The broadening by diffraction reflects the nanometer scale beam collimation in the waveguide exit plane. The diffraction broadening is directly evident when comparing the two measured photon energies, $7.9 \mathrm{keV}$ and $17.5 \mathrm{keV}$, respectively. Note that the same channel was measured in two consecutive experiments, the first at beamline ID2 $2 \mathrm{Ni}$ of ESRF at $17.5 \mathrm{keV}$, the second at $7.9 \mathrm{keV}$ at the nanofocus instrument GINIX at beamline P10/PETRA III at DESY. According to the requirements of photon energy in view of absorbing the radiation modes, the waveguide chip was cut to a length of $l=13 \mathrm{~mm}$ and $l=1 \mathrm{~mm}$, respectively. The comparison shows that the simulated farfield pattern is in good agreement with the experimental results shown in (a), and indeed agrees well after scaling the angular range according to the different photon energies.

In Fig. 6, experimental farfield diffraction patterns of waveguides with different channel sizes are compared. As the lateral size is decreased (in horizontal direction) fewer modes can propagate, leading to a less structured farfield. The farfield diffraction patterns were recorded at $13.8 \mathrm{keV}$ photon energy $5.2 \mathrm{~m}$ behind the waveguide by a Pilatus $300 \mathrm{~K}$ detector (Dectris) (GINIX-2 setting). The waveguide chip was fabricated according to procedure IV (see Table I) and provides therefore an enhanced etching depth of approximately $70 \mathrm{~nm}$. With comparable channel depths the reduction of the channel width leads to a noticeable change in the appearance of the farfield pattern. The recorded farfields exhibit a characteristic intensity pattern depending on the number of guides modes and hence the channel size. For broader channels (see Figs. 6(a) and 6(b)), the spatial frequencies in the farfield pattern along the horizontal direction are much higher than in vertical direction, since the confinement in the vertical direction is stronger than in the horizontal direction. 
This is in agreement with the calculation, which predicts a higher number of guided modes due to the larger channel width. With decreasing channel width, the frequency of the intensity modulation in horizontal direction approaches that of the vertical direction, i.e., the farfield becomes smoother and more isotropic (see Fig. 6(d)). With its intensity plateau in the center and an integrated waveguide flux of $I_{w g}=2.1 \times 10^{8} \mathrm{ph} / \mathrm{s}$, the waveguide shown in Fig. 6(d) is well suited for imaging applications. Further progress is directed towards equal width and depth and a smaller channel size, while providing a sufficiently high transmission by avoiding structural defects.

\section{OUTLOOK AND SUMMARY}

We have successfully fabricated and tested advanced two-dimensionally confining (channel) waveguides for hard $\mathrm{x}$-rays, using a processing scheme involving e-beam lithography, reactive ion etching and wafer bonding. The specific requirements are very demanding, in view of the high aspect ratios needed to filter out one or several guided modes, since the attenuation for the radiative modes in the silicon cladding requires a thickness of several millimeters. Hence the required aspect ratios of channel length and lateral structure size can easily exceed $10^{5}$, as in the present case for a $13 \mathrm{~mm}$ channel with a vertical cross section of $24 \mathrm{~nm}$. Only by using an interferometric stage in the e-beam lithography system, these specifications can be met. Apart from the high aspect ratios, the fact that the structures have to be buried in order to function as channel waveguides presents a second considerable challenge. As shown here, the wafer bonding process is suitable to cap the channels efficiently, but only after some optimization, in particular regarding the high temperature annealing step. In this work, we have attempted to minimize the considerable interfacial diffusion and reaction processes at high temperatures as these were considered to be detrimental to the channel shape. In the future, if these effects can be controlled more precisely, they could also be used to the benefit. In fact, a well defined set of propagating modes exists for any cross sectional form, and shapes differing from rectangular shapes may even be of advantage for some applications. More importantly, the annealing can lead to a desirable reduction in structure size. Unfortunately, this effect has so far not been uniform over the length of the channel, leading to transmission losses, probably by scattering from channel inhomogeneities. In this respect, one should briefly address the tolerance in fabrication. Channel waviness, defects and interface roughness are all deviations from the ideal (design) structure, which - however - affect the optical properties very differently. It is known that low frequency waviness and broadened interfacial profiles (i.e., as resulting from surface diffusion or surface reactions) do not impede the propagation of well defined modes. ${ }^{36}$ Contrarily, stitching errors and point defects can easily induce losses due to scattering of modes into the cladding. When traversing the full length of the structure, the scattered radiation is well absorbed in the cladding, and this effect will primarily lead to low transmission. Radiation scattered at higher angles, however, can escape the cladding, leading to the circular fringes around the central farfield cone, see Fig. 6(d). Importantly, due to the shallow angles of the internally reflected beam representing the modes, which are smaller than the critical angle of reflection $\alpha_{c}$ for silicon, the tolerance for defects (roughness, shape imperfections along the propagation direction) increases. ${ }^{36}$ This is the reason why waveguides can be fabricated for the hard x-ray range at all.

Here, we have presented an approach to waveguide nanofabrication based on e-beam lithography and wafer bonding. The waveguides fabricated by this method have been demonstrated to be suitable x-ray optical components for coherent imaging ${ }^{18}$ and nanodiffraction applications. ${ }^{47}$ Refinement of the process, in particular in terms of the annealing process and channel inspection is under way. Notably the approach allows for a large variation in waveguide types: both tapered waveguide profiles and beam splitters can be realized. In the future, adaptation of the bonding process may make germanium accessible, which is interesting in view of high photon energy applications. Bonding has also been reported for quartz, ${ }^{48}$ which would offer the opportunity of channel inspection with visible or infrared light.

\section{ACKNOWLEDGMENTS}

The authors would like to thank M. Reiche for helpful discussions on wafer bonding and K. Giewekemeyer for his contributions especially regarding the ID22Ni beamtime. Financial support by Deutsche Forschungsgemeinschaft through SFB 755 Nanoscale Photonic Imaging and by the Helmholtz society through Vernetzungsfonds VH-VI-403 is gratefully acknowledged.

${ }^{1}$ B. Niemann, D. Rudolph, and G. Schmahl, Opt. Commun. 12, 160 (1974).

${ }^{2}$ C. David, B. Nöhammer, T. Weitkamp, H. H. Solak, A. Diaz, and J. F. van der Veen, AIP Conf. Proc. 705, 764 (2004).

${ }^{3}$ W. Chao, B. D. Harteneck, J. A. Liddle, E. H. Anderson, and D. T. Attwood, Nature 435, 1210 (2005).

${ }^{4}$ C. G. Schroer, Phys. Rev. B 74, 033405 (2006).

${ }^{5}$ J. Maser, G. B. Stephenson, S. Vogt, W. Yun, A. Macrander, H. C. Kang, C. Liu, and R. Conley, Optical Science and Technology, the SPIE 49th Annual Meeting. International Society for Optics and Photonics, pp. 185-194 (2004).

${ }^{6}$ F. Döring, A.-L. Robisch, C. Eberl, M. Osterhoff, A. Ruhlandt, T. Liese, F. Schlenkrich, S. Hoffmann, M. Bartels, T. Salditt, and H.-U. Krebs, Opt. Express 21, 19311 (2013).

${ }^{7}$ A. Snigirev, V. Kohn, I. Snigireva, and B. Lengeler, Nature 384, 49 (1996).

${ }^{8}$ C. G. Schroer and B. Lengeler, Phys. Rev. Lett. 94, 054802 (2005).

${ }^{9}$ C. G. Schroer, O. Kurapova, J. Patommel, P. Boye, J. Feldkamp, B. Lengeler, M. Burghammer, C. Riekel, L. Vincze, A. V. D. Hart, and M. Kuchler, Appl. Phys. Lett. 87, 124103 (2005).

${ }^{10}$ P. Kirkpatrick and A. Baez, J. Opt. Soc. Am. 38, 766 (1948).

${ }^{11}$ O. Hignette, P. Cloetens, W.-K. Lee, W. Ludwig, and G. Rostaing, J. Phys. IV France 104, 231 (2003).

${ }^{12}$ E. Spiller and A. Segmüller, Appl. Phys. Lett. 24, 60 (1974).

${ }^{13}$ Y. Feng, S. Sinha, E. E. Fullerton, G. Grübel, D. Abernathy, D. Siddons, and J. Hastings, Appl. Phys. Lett. 67, 3647 (1995).

${ }^{14}$ S. Lagomarsino, W. Jark, S. Di Fonzo, A. Cedola, B. Mueller, P. Engström, and C. Riekel, J. Appl. Phys. 79, 4471 (1996).

${ }^{15}$ M. J. Zwanenburg, J. F. Peters, J. H. H. Bongaerts, S. A. de Vries, D. L. Abernathy, and J. F. van der Veen, Phys. Rev. Lett. 82, 1696 (1999).

${ }^{16}$ M. J. Zwanenburg, J. H. H. Bongaerts, J. F. Peters, D. O. Riese, and J. F. van der Veen, Phys. Rev. Lett. 85, 5154 (2000). 
${ }^{17}$ D. Pelliccia, I. Bukreeva, M. Ilie, W. Jark, A. Cedola, F. Scarinci, and S. Lagomarsino, Spectrochim. Acta, Part B 62, 615 (2007).

${ }^{18} \mathrm{~K}$. Giewekemeyer, H. Neubauer, S. Kalbfleisch, S. P. Krüger, and T. Salditt, New J. Phys. 12, 035008 (2010).

${ }^{19}$ A. Ruhlandt, T. Liese, V. Radisch, S. P. Krüger, M. Osterhoff, K. Giewekemeyer, H. U. Krebs, and T. Salditt, AIP Adv. 2, 012175 (2012).

${ }^{20}$ S. Di Fonzo, W. Jark, S. Lagomarsino, C. Giannini, L. De Caro, A. Cedola, and M. Müller, Nature 403, 638 (2000).

${ }^{21}$ M. Müller, M. Burghammer, D. Flot, C. Riekel, C. Morawe, B. Murphy, and A. Cedola, J. Appl. Crystallogr. 33, 1231 (2000).

${ }^{22}$ F. Pfeiffer, C. David, M. Burghammer, C. Riekel, and T. Salditt, Science 297, 230 (2002).

${ }^{23}$ A. Jarre, C. Fuhse, C. Ollinger, J. Seeger, R. Tucoulou, and T. Salditt, Phys. Rev. Lett. 94, 074801 (2005).

${ }^{24}$ C. Fuhse, C. Ollinger, and T. Salditt, Phys. Rev. Lett. 97, 254801 (2006).

${ }^{25}$ C. Bergemann, H. Keymeulen, and J. F. van der Veen, Phys. Rev. Lett. 91, 204801 (2003).

${ }^{26}$ H. Mimura, S. Handa, T. Kimura, H. Yumoto, D. Yamakawa, H. Yokoyama, S. Matsuyama, K. Inagaki, K. Yamamura, Y. Sano, K. Tamasaku, Y. Nishino, M. Yabashi, T. Ishikawa, and K. Yamauchi, Nat. Phys. 6, 122 (2010).

${ }^{27}$ S. Di Fonzo, W. Jark, G. Soullie, A. Cedola, S. Lagomarsino, P. Cloetens, and C. Riekel, J. Synchrotron Radiat. 5, 376 (1998).

${ }^{28}$ S. Eisebitt, J. Lüning, W. F. Schlotter, M. Lörgen, O. Hellwig, W. Eberhardt, and J. Stöhr, Nature 432, 885 (2004).

${ }^{29}$ G. J. Williams, H. M. Quiney, B. B. Dhal, C. Q. Tran, K. A. Nugent, A. G. Peele, D. Paterson, and M. D. de Jonge, Phys. Rev. Lett. 97, 025506 (2006).

${ }^{30}$ L. De Caro, C. Giannini, S. Di Fonzo, W. Jark, A. Cedola, and S. Lagomarsino, Opt. Commun. 217, 31 (2003).

${ }^{31}$ W. Jark and S. Di Fonzo, J. Synchrotron Radiat. 11, 386 (2004).
${ }^{32}$ I. Bukreeva, A. Popov, D. Pelliccia, A. Cedola, S. B. Dabagov, and S. Lagomarsino, Phys. Rev. Lett. 97, 184801 (2006).

${ }^{33}$ C. Fuhse and T. Salditt, Appl. Opt. 45, 4603 (2006).

${ }^{34}$ C. Fuhse and T. Salditt, Opt. Commun. 265, 140 (2006).

${ }^{35}$ S. Panknin, A. K. Hartmann, and T. Salditt, Opt. Commun. 281, 2779 (2008).

${ }^{36}$ M. Osterhoff and T. Salditt, Opt. Commun. 282, 3250 (2009).

${ }^{37}$ S. P. Krüger, K. Giewekemeyer, S. Kalbfleisch, M. Bartels, H. Neubauer, and T. Salditt, Opt. Express 18, 13492 (2010).

${ }^{38}$ M. Poulsen, F. Jensen, O. Bunk, R. Feidenhans'l, and D. W. Breiby, Appl. Phys. Lett. 87, 261904 (2005).

${ }^{39}$ A. Kohlstedt, S. Kalbfleisch, T. Salditt, M. Reiche, U. Gösele, E. Lima, and P. Willmott, Appl. Phys. A 91, 7 (2008).

${ }^{40}$ U. Gösele and Q.-Y. Tong, Annu. Rev. Mater. Sci. 28, 215 (1998).

${ }^{41}$ B. Cord, J. Lutkenhaus, and K. K. Berggren, J. Vac. Sci. Technol. B 25, 2013 (2007).

${ }^{42}$ X. X. Zhang and J.-P. Raskin, J. Microelectromech. Syst. 14, 368 (2005)

${ }^{43}$ W. Kern and D. Puotinen, RCA Rev. 31, 187 (1970).

${ }^{44}$ W. P. Maszara, G. Goetz, A. Caviglia, and J. B. McKitterick, J. Appl. Phys. 64, 4943 (1988).

${ }^{45}$ S. Kalbfleisch, M. Osterhoff, K. Giewekemeyer, H. Neubauer, S. P. Krüger, B. Hartmann, M. Bartels, M. Sprung, O. Leupold, F. Siewert, and T. Salditt, AIP. Conf. Proc. 1234, 433 (2010).

${ }^{46}$ S. Kalbfleisch, H. Neubauer, S. P. Krüger, M. Bartels, M. Osterhoff, D. D. Mai, K. Giewekemeyer, B. Hartmann, M. Sprung, and T. Salditt, AIP Conf. Proc. 1365, 96 (2011).

${ }^{47}$ C. Krywka, H. Neubauer, M. Priebe, T. Salditt, J. Keckes, A. Buffet, S. V. Roth, R. Döhrmann, and M. Müller, J. Appl. Crystallogr. 45, 85 (2012).

${ }^{48}$ A. Grosse, M. Grewe, and H. Fouckhardt, J. Micromech. Microeng. 11, 257 (2001). 


\section{Chapter 3}

\section{Advances in $x$-ray waveguide fabrication}

Reprinted from

S. Hoffmann-Urlaub, P. Höhne, M. Kanbach and T. Salditt Advances in $x$-ray waveguide fabrication,

Microelectronic Engineering 164, 135-138, (2016)

with permission from Elsevier. 


\title{
Advances in fabrication of X-ray waveguides
}

\author{
Sarah Hoffmann-Urlaub*, Philipp Höhne, Mike Kanbach, Tim Salditt \\ Institut für Röntgenphysik, Universität Göttingen, Friedrich-Hund Platz 1, 37077 Göttingen, Germany
}

\section{A R T I C L E I N F O}

\section{Article history:}

Received 25 May 2016

Received in revised form 20 June 2016

Accepted 26 July 2016

Available online 4 August 2016

\section{Keywords:}

X-ray imaging

Coherence

Wafer bonding

Electronbeam lithography

Reactive ion etching

X-ray waveguides

\begin{abstract}
A B S T R A C T
This paper reports on the fabrication of X-ray waveguides, manufactured by e-beam lithography, reactive ion etching and wafer bonding techniques. By combination of these processing steps, long empty (air) channels with cross-sections in the range of 10 to $100 \mathrm{~nm}$ are obtained, forming a guiding layer, surrounded by a solid state cladding. Aside from silicon, we present also waveguide channels fabricated in germanium and quartz. The improved fabrication protocols lead to significantly enhanced exit flux for imaging applications. Finally, we address not only straight channels, but a large variety of various geometries, as required for different applications. (c) 2016 Elsevier B.V. All rights reserved.
\end{abstract}

\section{Introduction}

While waveguide (WG) optics is ubiquitous in the visible and infrared spectral range, it is still much less developed in the X-ray regime, despite some encouraging initial work on planar X-ray WGs [1-8]. This is for a good reason. The guiding channel cross-sections in the range of 10 to $100 \mathrm{~nm}$ and the required length to reach well defined propagation regimes, are extremely challenging in view of fabrication. As in other Xray focusing optics, such as Fresnel zone plates [9-11] or compact refractive lenses [12-14], the combination of small wavelength and vanishing differences in the index of refraction $n \approx 1$, leads to areas and aspect ratios which are very different from their counterparts in visible light optics. Ideally, an X-ray WG consist of an empty (air filled) channel, embedded in a moderately absorbing material (cladding), with the entrance (front) and exit sides of the channel left open for coupling and decoupling of guided beam. Hence, an X-ray WG essentially corresponds to a long empty fiber defined in a solid body. For example, typical hard X-ray photon energies $\left(E_{p h}>10 \mathrm{keV}\right)$ require a significant propagation length in the channel to sufficiently block radiative modes in the cladding. The aspect ratios are hence in the range of $1: 10^{4}-10^{5}$. The long channel length results in multiple internal reflections increasing the probability of the X-rays to be scattered by defects or side wall roughness [15]. To avoid leakage and over-illumination also the cladding has to be sufficiently thick, resulting in 'buried' channels. Recent progress in fabrication has helped to meet these challenges [16], and have enabled promising applications in nanoscale holographic X-ray imaging $[17,18]$. The experiments took advantages of the optical

\footnotetext{
* Corresponding author

E-mail address: shoffma3@gwdg.de (S. Hoffmann-Urlaub).
}

properties of X-ray WGs in confining and filtering synchrotron radiation and of the quasi-point source illumination which they can provide for cone beam holographic imaging. Nano-manufacturing of WGs combines different fabrication steps and techniques, namely e-beam lithography (EBL), reactive ion etching and wafer bonding. As a result of the high flexibility of these process steps, a multitude of channel geometries is feasible. In analogy to lab-on-a-chip approaches, this could become a strategy for X-ray optics on a chip [19]. In practice, such integrated X-ray nano-optics will be limited to a large extent by fabrication issues. Since the guiding effect of the WGs is based on total reflection, smooth channel boundaries are required. To this end, a precisely controlled fabrication process is needed. The optimized protocols as presented here now offer better control of the channel cross-section, and enhanced smoothness of the side walls, leading in turn to better X-ray optical performance. Apart from straight WGs [16], we also address fabrication of WGs with a tapered geometry for beam concentration [20], as well as curved channels and WG arrays. Finally, we broaden the range of materials, beyond the channels in Si presented in [16], also to quartz $\left(\mathrm{SiO}_{2}\right)$ and $\mathrm{Ge}$. The latter will be particularly useful for high photon energies.

\section{Experimental methods}

Wafers of $\mathrm{Si}$ (University Wafer), Ge (MaTeck) and $\mathrm{SiO}_{2}$ (Quartz Unlimited LLC) each with a diameter of 4 in. were covered by an e-beam resist (ARP 679.04, Allresist), consisting of a poly-methyl methacrylate (PMMA) solution. The resist was spin coated (Optispin SB20, Sister Semiconductor Equipment) onto the wafer at $2000 \mathrm{rpm}$ for $70 \mathrm{~s}$, followed by a post baking step $\left(180{ }^{\circ} \mathrm{C}\right.$ for $\left.90 \mathrm{~s}\right)$. In the case of $\mathrm{SiO}_{2}$, an additional resist (SX AR-PC 5000/90.2, Allresist) was used to enable EBL (e-LiNE, Raith) on the insulating substrate. After spin-coating at 
$2000 \mathrm{rpm}$ for $60 \mathrm{~s}$ the associated baking step of $120 \mathrm{~s}$ at $90{ }^{\circ} \mathrm{C}$ was performed. Arrays of lines and curves were exposed by EBL. For lines wider than $300 \mathrm{~nm}$ an area dose of $200 \mathrm{C} / \mathrm{cm}^{2}$ and for smaller ones $600 \mathrm{C} / \mathrm{cm}^{2}$ was chosen. Single pixel lines were exposed with $3000 \mathrm{pC} / \mathrm{cm}$. The $\mathrm{SiO}_{2}$ was plunged into deionized water for $60 \mathrm{~s}$ to clean the sample from the conductive resist. To remove the illuminated resist from all samples, a development procedure with a mixture of Methyl isobutyl ketone and 2-Propanol (IPA) with a ratio of $1: 3$ at $0{ }^{\circ} \mathrm{C}$ for $30 \mathrm{~s}$ was carried out [21]. The process was stopped by immersing the sample in pure IPA at room temperature for $30 \mathrm{~s}$. Afterwards the pattern was transferred to the wafer by reactive ion etching (PlasmaLab, Oxford) employing $\mathrm{CHF}_{3}$ for sidewall passivation [22]. The details for the subsequent fabrication steps are listed in Table 1.

The etching step was performed according to the parameters listed in Table 1. Afterwards, the resist was removed either using an RCA solution for $15 \mathrm{~min}$ at a temperature of $65^{\circ} \mathrm{C}$ or $\mathrm{HCl}$ at room temperature for $10 \mathrm{~min}$. Each of the structured wafers was manually covered with a second wafer of the same material. Instantly, the wafers are kept together by van-der-Waals bonds which are transformed to covalent bonds during the wafer bonding process [24] in an oven (L 3/11/P330, Nabertherm) under a $\mathrm{N}_{2}$ atmosphere (flow rate $50 \mathrm{l} / \mathrm{h}$ ). Since the melting point of $\mathrm{Ge}\left(937^{\circ} \mathrm{C}\right)$ is lower than for $\mathrm{Si}\left(1412^{\circ} \mathrm{C}\right)$ [25], a decreased bonding temperature $T_{b}$ was chosen accordingly. At higher temperatures, channels with a size in the range of $100 \times 100 \mathrm{~nm}^{2}$ were closed due to diffusion processes, which was also the case for $\mathrm{SiO}_{2}$. The chips were cut to the chosen geometry (DAD320, Disco), leaving the entrance and exit sides of the channels open. For Si, an infrared camera (XEVA XS Base, Xenics) enabled the inspection of the embedded structures from a top view perspective. For inspection of the $\mathrm{SiO}_{2}$ channels, a fluorescence microscope (Axio Observer Z1, Zeiss) was used, after filling the channels by capillary suction with an aqueous solution of fluorescein (Uranin, $\mathrm{C}_{20} \mathrm{H}_{10} \mathrm{Na}_{2} \mathrm{O}_{5}$ at $\left.100 \mathrm{mM}[26]\right)$.

The manufacturing sequence described above corresponds to the optimized protocol. The main difference to the previously published protocol for Si WG fabrication [16] is in replacing the formerly used PMMA resist (A2, MicroChem) by a PMMA resist of higher viscosity, which in turn entails small modifications of the parameters in the following processing steps (spin-coating, e-beam lithography, etching), as described above. The main advantage of this replacement is a higher resist film thickness. The previous protocol enabled a film thickness of only $100 \mathrm{~nm}$, as obtained for spin-coating at $2000 \mathrm{rpm}$ for $60 \mathrm{~s}$. This limited the etching time to $70 \mathrm{~s}$, before the resist was completely consumed. The improved protocol now enables a larger range of etching times and correspondingly larger range of etching depths, as tabulated above. A second important difference is that we now refrain from a cleaning step, in which the wafers were exposed to an $\mathrm{O}_{2}$ plasma up to $30 \mathrm{~min}$. This was intended to achieve a hydrophilic surface in order to enhance bonding strength. However, the oxygen gassed out in the oven resulting in unwanted bubbles at the bonding interface, as further detailed below.

Table 1

Processing parameters for etching, cleaning and wafer bonding steps for the different materials.

\begin{tabular}{|c|c|c|c|c|c|c|}
\hline \multirow[t]{3}{*}{ Wafer } & \multicolumn{3}{|l|}{ Etching } & \multirow[t]{3}{*}{ Cleaning procedure } & \multicolumn{2}{|c|}{ Bonding } \\
\hline & Gases & $t_{e}$ & Depth & & $T_{b}$ & $t_{b}$ \\
\hline & {$[\mathrm{sccm}]$} & {$[s]$} & {$[\mathrm{nm}]$} & & {$\left[{ }^{\circ} \mathrm{C}\right]$} & {$[\mathrm{h}]$} \\
\hline $\mathrm{Si}$ & $\mathrm{SF}_{6}: \mathrm{CHF}_{3} / 40: 10$ & 160 & 400 & $\mathrm{RCA}^{\mathrm{a}}$ & 1000 & 4 \\
\hline $\mathrm{Ge}$ & $\mathrm{SF}_{6}: \mathrm{CHF}_{3} / 40: 10$ & 160 & 320 & $\mathrm{HCl}$ & 650 & 4 \\
\hline Quartz & $\mathrm{CF}_{4}: \mathrm{CHF}_{3} / 20: 30$ & 590 & 160 & DI- $\mathrm{H}_{2} \mathrm{O} / \mathrm{RCA}$ & 550 & 12 \\
\hline
\end{tabular}

${ }^{\text {a }}$ A mixture of ammonia (32\%), hydrogen peroxide (30\%) and deionized water (1:1:5) [23].

\section{Results and discussion}

To characterize the WGs, light microscopy (LM), scanning electron microscopy (SEM), infrared (IR) microscopy and finally X-ray nano-optical testing were performed. Several Si channels are depicted in Fig. 1 to illustrate typical geometries.

Fig. 1 (a) shows a LM image of a typical X-ray WG array, both with large channels for alignment and small ones, which confine and guide the beam in both lateral axis. The $\mathrm{Si}$ is covered with the e-beam resist which also serves as a mask during the etching process. Channels broader than $300 \mathrm{~nm}$ are silhouetted against the homogeneous background as bright lines, while the narrower ones appear darker.

In (b,c) IR transmission images of bonded wafer-pairs are depicted. Round or elliptically shaped dark spots accompanied by interference fringes are observed, indicative of voids between the two wafers [27]. These unbonded regions or embedded gas reservoirs can be caused by trapped gas, imperfections on the wafers surface or by particles. In this work, the dominating effect was gas bubbles originating from molecules desorbing from the wafers during annealing. Usually the gas consists of water or hydrocarbons [28], which at high temperature can only be dissolved in small amounts in the bulk Si or the interfacial oxide layer. Micro-defects at the surface then serve as nucleation sites for bubble formation [29], as is frequently observed after oxygen plasma treatment [30]. For this reason, plasma cleaned wafers are often found to be highly covered with voids (b), affecting the local bonding quality. Substituting the plasma cleaning by an RCA bath decreases the number of (small) bubbles significantly, while hardly reducing the bonding strength between the wafers [31]. Contamination is identified as the dominant mechanism of generating large bubbles at the RCA cleaned wafers, since micron-sized particles induce voids with a diameter up to several centimeters. Therefore, in (c) mainly bubbles with diameters $\geq 0.5 \mathrm{~cm}$ are observed, which can in many cases be removed

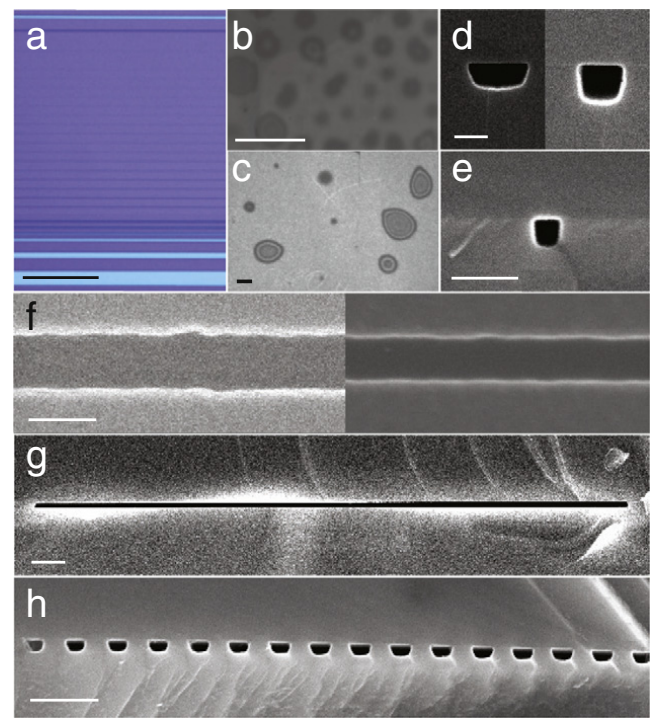

Fig. 1. Structured Si wafers. (a) LM top view of a WG chip after development, before etching. Scale bar denotes $100 \mathrm{~m}$. (b,c) IR images of bubbles between bonded wafers, processed according to the old (b) and new (c) protocol. Scale bars denote $5 \mathrm{~mm}$. (d,e) SEM images of the exit planes of final WG channels and (f) a top view of an unbonded (open) channel. In $(\mathrm{d}, \mathrm{f})$ the left part shows the old protocol and the right the new one. Scale bars denote $100 \mathrm{~nm}$. (g,h) SEM images of exit planes of final channels, showing ( $g$ ) a channel with a broad entrance (for beam compression by tapering), and ( $h$ ) an array of WG channels. Scale bars denote $1 \mu \mathrm{m}$. 
by pushing them manually towards the wafers edges (see [16], Fig. 3) or by repeating the cleaning procedure to detach the remaining particles.

Examples for WGs after the final dicing step are depicted in (d). The channel on the left side is fabricated according to the old and the right channel to the new protocol, exhibiting cross-sectional areas of $169 \times 75 \mathrm{~nm}^{2}$ and $125 \times 121 \mathrm{~nm}^{2}$, respectively. The lack of depth is due to the small resist thickness $(100 \mathrm{~nm})$, limiting the maximum etching depth. While the left one reveals a trapezoidal shape, the right one is almost quadratically for approximately the same channel width. The shape difference can be attributed to the proximity effect, where electrons scattered within the resist or backwards from the substrate broaden the (partially) illuminated area. This effect has more impact if the resist is thin.

Smaller channels in the range of $38 \times 42 \mathrm{~nm}^{2}$ were also fabricated, still showing smooth and well defined boundaries, as evidenced by (e). Inside the chip, the channels walls are well defined, as probed in the unbound state (see ( $\mathrm{f}$, right)), even if some residual roughness is visible.

As depicted in (d, left), for the old protocol the channels walls are not very steep. The corresponding slope appears as a bright stripe in the top view of (f, left). As can be observed, the new protocol not only steepens the slope, but also reduces the interfacial roughness (f, right).

The evolution of the channel roughness during the bonding process is unclear, but it is likely that some smoothing occurs due to surface mobility. Apart from small single WGs, large entrances, for example with $17 \mu \mathrm{m}$ in size (g), or gratings of equally spaced channels (h) can be manufactured, enabling WGs in a tapered geometry [20], as well as Xray probes which are modified/structured on the nanometer scale.

Following the continuous inspection by LM and SEM along the fabrication process, the functional characterization requires nano-focused Xray optics using synchrotron radiation, to actually evidence the successful WG operation. To this end, we have previously established suitable pre-focusing of the synchrotron beam onto the channels entrance, as well as the measurements of the far-field diffraction pattern several meters behind the WG optics [16,17]. Such data allows to determine transmission, numerical aperture, suitability for holographic imaging, and by inversion techniques also the near-field distribution and hence the beam confinement. Fig. 2 shows results for different WG geometries on logarithmic scale: (a) The far-field pattern (intensity distribution)
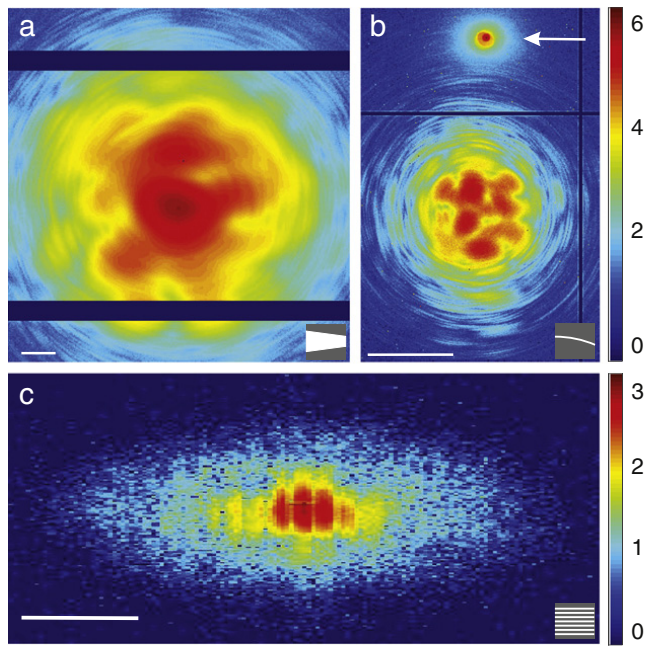

Fig. 2. Far-field patterns of WGs in tapered (a), curved (b) and grating (c) geometry. At the bottom right, corresponding schematics of the channel path are included (X-rays entering from the left side). The images are logarithmically scaled, scale bars denote $5 \mathrm{~mm}$. of a tapered channel with $319 \times 142 \mathrm{~nm}^{2}$ and $83 \times 96 \mathrm{~nm}^{2}$ as entrance and exit size, respectively. The exit flux of the WG is $I=1.45 \cdot 10-$

${ }^{10}$ photons/s, indicating successful beam compression by tapering. While the tails of the beam shows a structured (multi-modal) regime, the far-field is homogeneous in the central region, which qualifies the channel for applications in coherent (holographic) X-ray imaging. The dark horizontal stripes are the gaps between different detector modules. The measurement was performed at the GINIX (Göttingen Instrument for Nano-Imaging with X-rays [32]) endstation (P10 beamline at DESY, Hamburg) at $E_{p h}=7.9 \mathrm{keV}$. The CCD detector (Pilatus $300 \mathrm{k}$, DECTRIS) was positioned $5.1 \mathrm{~m}$ behind the WG chip, with the X-rays propagating about $1 \mathrm{~m}$ in air before entering an evacuated tube through a Be foil.

Fig. 2 (c) shows the far-field of the WG array displayed in Fig. 1(f), recorded at the BM20 beamline (ESRF, France) with a Pilatus $100 \mathrm{k}$ (DECTRIS) at a distance of $4.0 \mathrm{~m}$ and $E_{p h}=11.5 \mathrm{keV}$. The vertical stripes result from the interference of the 20 neighbouring channels $\left(164 \times 97 \mathrm{~nm}^{2}\right)$, aligned in the horizontal direction. Designing such structured beams consisting of multiple secondary sources can help to improve reconstruction techniques for coherent X-ray imaging. In [33], we have observed that for such an illumination (probe) system, the reconstruction of the probe by iterative algorithms (error reduction), was more robust against an incorrect initialization than the conventional (single-exit) channel.

A further desirable functionality of X-ray WGs is guiding in curved geometries. In this way, X-rays could be redirected and transported to different exit locations, as is commonplace for visible light. Contrarily, a limiting factor in terms of guiding X-rays is the glancing critical angle of total reflection $\left(\alpha_{c}=0.224^{\circ}\right.$ for $E_{p h}=8 \mathrm{keV}$ [34]). It was first shown by Liu and Golovchenko [35] that surface modes of a bent Si crystal, can be utilized to achieve bending over much larger angles, exploiting multiple reflections. Recently, we have used lithographed, sub-micrometer sized, channels in Ta to deflect the beam up to unprecedented values of $30^{\circ}$ at $E_{p h}=8 \mathrm{keV}$ [19]. In that work the channels were open at the top side (uncapped channels), resulting in some leakage. Here we also demonstrated guiding in capped and (mildly) curved WGs in bonded $\mathrm{Si}$, illustrating the versatility of the present fabrication scheme. To this end, Fig. 2(b) shows the far-field of a curved channel in Si capped by wafer bonding, as recorded at $E_{p h}=8 \mathrm{keV}$ at the ID01 beamline (ESRF, France) with a Maxipix detector (ESRF) $0.95 \mathrm{~m}$ behind the WG chip. Note that the dark straight lines correspond to the gaps between the detector modules. The curvature (radius $R=200 \mathrm{~mm}$ ) of the WG leads to a deflection of $0.6^{\circ}$ with respect to the primary beam, which is indicated by an arrow. Using curved or slightly inclined elements, two beams can be separated [36] or one beam can be split into sub-beams [33].

Compared to the old fabrication protocol the transmission was increased from $T<5 \%$ to $T=20 \%$ for straight WGs. With curved channels values up to $T=13 \%$ were achieved (for $R=500 \mathrm{~mm}$ ), beamsplitters and gratings reached $T=30 \%$ and tapered WGs exceeded this to $T=$ $40 \%$.

Next, the fabrication process was successfully transferred from Si to $\mathrm{Ge}$ and $\mathrm{SiO}_{2}$. For $\mathrm{Ge}$, similar channels sizes and side-wall smoothness were achieved as for Si (compare Figs. 2(d) and 3(a)). For the etching conditions listed in Table 1, Ge has a slightly lower etching rate than $\mathrm{Si}$, resulting in a decrease of the maximum etching depth from $400 \mathrm{~nm}(\mathrm{Si})$ to $320 \mathrm{~nm}(\mathrm{Ge})$. Since desired channel cross-sections are mostly smaller, this does not represent a severe restriction. For $\mathrm{SiO}_{2}$, the mask was already consumed before reaching an etching depth of $100 \mathrm{~nm}$. Hence the etching gas was substituted, resulting in an maximum channel depth of $180 \mathrm{~nm}$. In Fig. 3(b), channels with widths between $20 \mu \mathrm{m}$ and $41 \mathrm{~nm}$ are depicted. For clarity, the front end of the WG chip is highlighted by a dashed line and in addition, every second of the channels smaller than $100 \times 100 \mathrm{~nm}^{2}$ is marked by an arrow. The fluorescent molecules are homogeneously distributed within the channels and thus provide a high contrast of the bright channels with 

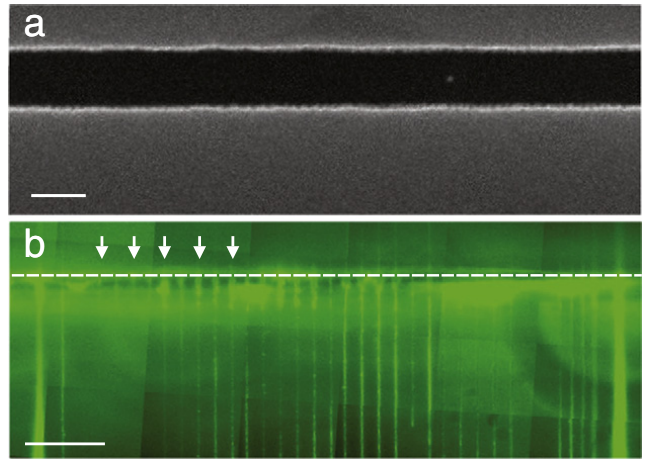

Fig. 3. Channels in $\mathrm{Ge}$ and $\mathrm{SiO}_{2}$. SEM top view of an unbonded channel in Ge. Scale bar denotes $100 \mathrm{~nm}$. Stitched fluorescence image of channels embedded in $\mathrm{SiO}_{2}$. Scale bar denotes $150 \mu \mathrm{m}$

respect to the dark $\mathrm{SiO}_{2}$. Due to capillary forces, the channels are instantly filled with the Uranin when the open endings are dipped into the solution.

\section{Conclusion}

EBL, reactive ion etching and wafer bonding at optimized processing parameters now enable the fabrication of a large variety X-ray WG geometries, beyond the simple straight WGs [16]. This includes tapered, curved and multiple beam arrays. The flexibility in design and fabrication can serve firstly to achieve higher exit fluxes while keeping the exit source sizes small (tapered WGs), as desired for high resolution imaging. Secondly, guiding in curved channel segments opens up new tools to redirect one beam, or several beams as required for reference beam holography. Thirdly, curved split and delay lines could be used for X-ray pulse splitting at ultra-short time scales. Finally, the advanced X-ray WG optics presented here provide well designed probes for phase retrieval algorithms. In all cases, the aspect ratio of the WG channels, i.e. the ratio between length and cross-section is extremely high, up to $10^{5}$, making them quite unique nanostructures. Importantly, a refined fabrication protocol (concerning in particular cleaning and bonding) has also led to an improved channel quality. This has paved the way to obtain an exit flux exceeding $10^{10} \mathrm{X}$-ray photons/s in a WG channel with beam confinement in both lateral dimensions. Furthermore, the fabrication process was successfully translated to $\mathrm{Ge}$ and $\mathrm{SiO}_{2}$, achieving channels of the same quality as already documented for $\mathrm{Si}$.

\section{Acknowledgments}

We thank Dr. Hsin-Yi Chen for her help with the tapered Si WGs, Ferdinand Döring for supporting the Ge experiments and Susanne Hengst for her assistance at the fluorescence microscope. For the excellent technical support at the beamlines we want to thank Michael Sprung (P10, DESY), Jan Hilhorst (ID01, ESRF) and Carsten Baehtz (BM20, ESRF) and the Helmholtz Society for funding through grant VH-VI-403.

\section{References}

[1] E. Spiller, A. Segmüller, Propagation of X-rays in waveguides, Appl. Phys. Lett. 24 (2) (1974) 60-61.

[2] Y. Feng, S. Sinha, E.E. Fullerton, G. Grübel, D. Abernathy, D. Siddons, J. Hastings, X-ray Fraunhofer diffraction patterns from a thin-film waveguide, Appl. Phys. Lett. 67 (24) (1995) 3647-3649.

[3] S. Lagomarsino, W. Jark, S. Di Fonzo, A. Cedola, B. Mueller, P. Engström, C. Riekel, Submicrometer X-ray beam production by a thin film waveguide, J. Appl. Phys. 79 (8) (1996) 4471-4473.
[4] M. Zwanenburg J. Peters, J. Bongaerts, S. De Vries, D. Abernathy, J. Van der Veen, Coherent propagation of X-rays in a planar waveguide with a tunable air gap, Phys. Rev. Lett. 82 (8) (1999) 1696

[5] F. Pfeiffer, T. Salditt, P. Høghøj, I. Anderson, N. Schell, X-ray waveguides with multiple guiding layers, Phys. Rev. B 62 (24) (2000) 16939.

[6] I. Prudnikov, X-ray waveguides based on Bragg scattering of multilayers, Phys. Rev. B 67 (23) (2003) 233303.

[7] J. Bongaerts, M. Zwanenburg, F. Zontone, J. van der Veen, Propagation of coherent X rays in a multistep-index X-ray waveguide, J. Appl. Phys. 90 (1) (2001) 94-100.

[8] D. Pelliccia, A. Cedola, F. Scarinci, S. Lagomarsino, X-ray waveguides for laboratory sources, J. Phys. D. Appl. Phys. 38 (10A) (2005) A213.

[9] G. Schmahl, D. Rudolph, Lichtstarke Zonenplatten als abbildende Systeme für weiche Röntgenstrahlung, Optik 29 (6) (1969) 577-585.

[10] F. Seiboth, M. Scholz, J. Patommel, R. Hoppe, F. Wittwer, J. Reinhardt, J. Seidel, M. Knaut, A. Jahn, K. Richter, J.W. Bartha, G. Falkenberg, C.G. Schroer, Hard X-ray nanofocusing by refractive lenses of constant thickness, Appl. Phys. Lett. 105 (13) (2014)

[11] P. Karvinen, D. Grolimund, M. Willimann, B. Meyer, M. Birri, C. Borca, J. Patommel, G. Wellenreuther, G. Falkenberg, M. Guizar-Sicairos, A. Menzel, C. David, Kinoform diffractive lenses for efficient nano-focusing of hard X-rays, Opt. Express 22 (14) (2014) 16676-16685.

[12] A. Snigirev, V. Kohn, I. Snigireva, B. Lengeler, et al., A compound refractive lens for focusing high-energy X-rays, Nature 384 (6604) (1996) 49-51.

[13] C.G. Schroer, Focusing hard X-rays to nanometer dimensions using Fresnel zone plates, Phys. Rev. B 74 (3) (2006) 033405.

[14] J. Vila-Comamala, S. Gorelick, E. Färm, C.M. Kewish, A. Diaz, R. Barrett, V.A. Guzenko, M. Ritala, C. David, Ultra-high resolution zone-doubled diffractive X-ray optics for the multi-kev regime, Opt. Express 19 (1) (2011) 175-184.

[15] M. Osterhoff, T. Salditt, Real structure effects in X-ray waveguide optics: the influence of interfacial roughness and refractive index profile on the near-field and farfield distribution, Opt. Commun. 282 (16) (2009) 3250-3256.

[16] H. Neubauer, S. Hoffmann, M. Kanbach, J. Haber, S. Kalbfleisch, S. Krüger, T. Salditt, High aspect ratio X-ray waveguide channels fabricated by e-beam lithography and wafer bonding, J. Appl. Phys. 115 (21) (2014) 214305.

[17] M. Bartels, M. Krenkel, J. Haber, R. Wilke, T. Salditt, X-ray holographic imaging of hydrated biological cells in solution, Phys. Rev. Lett. 114 (2015) 048103.

[18] M. Krenkel, A. Markus, M. Bartels, C. Dullin, F. Alves, T. Salditt, Phase-contrast zoom tomography reveals precise locations of macrophages in mouse lungs, Sci. Rep. 5 (2015) 9973.

[19] T. Salditt, S. Hoffmann, M. Vassholz, J. Haber, M. Osterhoff, J. Hilhorst, X-ray optics on a chip: guiding X rays in curved channels, Phys. Rev. Lett. 115 (2015) 203902.

[20] H. Chen, S. Hoffmann, T. Salditt, X-ray beam compression by tapered waveguides, Appl. Phys. Lett. 106 (19) (2015) 194105.

[21] B. Cord, J. Lutkenhaus, K. Berggren, Optimal temperature for development of poly(methylmethacrylate), J. Vac. Sci. Technol. B 25 (6) (2007) 2013-2016.

[22] R. Legtenberg, H. Jansen, M. de Boer, M. Elwenspoek, Anisotropic reactive ion etching of silicon using $\mathrm{SF}_{6} / \mathrm{O}_{2} / \mathrm{CHF}_{3}$ gas mixtures, J. Electrochem. Soc. 142 (6) (1995) 2020-2028.

[23] K. Kern, A. Werner (Eds.), Handbook of Silicon Wafer Cleaning Technology, second ed.William Andrew Publishing, 2008.

[24] U. Gösele, M. Reiche, H. Stenzel, T. Martini, H. Steinkirchner, Q.-Y. Tong, History and Future of Semiconductor Wafer Bonding, in: Gettering and Defect Engineering in Semiconductor Technology VI, Vol. 47 of Solid State Phenomena, Trans Tech Publications 1995, pp. 33-44

[25] S. Pizzini, Physical Chemistry of Semiconductor Materials and Processes, John Wiley \& Sons, 2015.

[26] O. Lautenschläger, Carl Roth GmbH + Co. KG., Spezifikationen Fluorescein-Natrium, 2015.

[27] T. Piotrowski, W. Jung, Characterization of silicon wafer bonding by observation in transmitted infrared radiation from an extended source, Thin Solid Films 364 (1) (2000) 274-279.

[28] K. Mitani, V. Lehmann, U. Gosele, Bubble Formation during Silicon Wafer Bonding: Causes and Remedies, Solid-State Sensor and Actuator Workshop, 1990. 4th Technical Digest. IEEE 1990, pp. 74-77.

[29] X.X. Zhang, J.-P. Raskin, Low-temperature wafer bonding: a study of void formation and influence on bonding strength, J. Microelectromech. Syst. 14 (2) (2005) 368-382.

[30] O. Zucker, W. Langheinrich, M. Kulozik, H. Goebel, Application of oxygen plasma processing to silicon direct bonding, Sensors Actuators A Phys. 36 (3) (1993) 227-231.

[31] M. Wiegand, M. Reiche, U. Gösele, Time-dependent surface properties and wafe bonding of $\mathrm{O}_{2}$-plasma-treated silicon (100) surfaces, J. Electrochem. Soc. 147 (7) (2000) 2734-2740.

[32] T. Salditt, M. Osterhoff, M. Krenkel, R.N. Wilke, M. Priebe, M. Bartels, S. Kalbfleisch, M. Sprung, Compound focusing mirror and X-ray waveguide optics for coherent imaging and nano-diffraction, J. Synchrotron Radiat. 22 (4) (2015) 867-878.

[33] S. Hoffmann-Urlaub, T. Salditt, Miniaturized Beamsplitters Realized by X-ray Waveguides, Acta Crystallogr. Section A72 (2016), http://dx.doi.org/10.1107/ S205327331601144X (in press).

[34] B. Henke, E. Gullikson, J. Davis, X-ray interactions: photoabsorption, scattering transmission, and reflection at $\mathrm{E}=50-30,000 \mathrm{eV}, \mathrm{Z}=1-92$. At. Data Nucl. Data Tables 54 (2) (1993) 181-342.

[35] C. Liu, J.A. Golovchenko, Surface trapped X-rays: whispering-gallery modes at $\lambda=$ $0.7 \AA$ A, Phys. Rev. Lett. 79 (5) (1997) 788.

[36] C. Fuhse, C. Ollinger, T. Salditt, Waveguide-based off-axis holography with hard X rays, Phys. Rev. Lett. 97 (25) (2006) 254801. 



\section{Chapter 4}

\section{X-ray beam compression by tapered waveguides}

Reprinted from

H.-Y. Chen, S. Hoffmann and T. Salditt

$X$-ray beam compression by tapered waveguides

Applied Physics Letters, 106(19), 194105 (2015)

with the permission of AIP Publishing. 


\title{
X-ray beam compression by tapered waveguides
}

\author{
H.-Y. Chen (陳心誼), ${ }^{\text {a) }}$ S. Hoffmann, and T. Salditt ${ }^{\text {a) }}$ \\ Institut für Röntgenphysik, Universität Göttingen, Friedrich-Hund-Platz 1, 37077 Göttingen, Germany
}

(Received 6 February 2015; accepted 2 May 2015; published online 12 May 2015)

\begin{abstract}
We have fabricated linear tapered waveguide channels filled with air and imbedded in silicon for the hard x-ray regime, using a processing scheme involving e-beam lithography, reactive ion etching, and wafer bonding. Beam compression in such channels is demonstrated by coupling a pre-focused undulator beam into the channels, and recording the exit flux and far-field diffraction patterns. We achieved a compressed beam with a spot size of $16.48 \mathrm{~nm}$ (horizontal) $\times 14.6 \mathrm{~nm}$ (vertical) near the waveguide exit plane, as determined from the reconstructed near-field distribution, at an exit flux which is eight times higher than that of an equivalent straight channel. Simulations indicate that this gain could reach three to four orders of magnitude for longer channels with tapering in two directions. (C) 2015 AIP Publishing LLC.
\end{abstract}

[http://dx.doi.org/10.1063/1.4921095]

X-ray waveguides (WGs) can be used to generate quasipoint sources for hard $\mathrm{X}$-ray imaging. ${ }^{1}$ These kinds of $\mathrm{X}$-ray optics have first been realized as planar layered systems [one-dimensional waveguide (1DWG),$^{2-5}$ and later also as two-dimensional confined channels (2DWGs), ${ }^{6,7}$ as required for applications in coherent imaging. In contrast to most other nano-focusing optics such as Kirkpatrick-Baez (KB) mirrors, ${ }^{8-11}$ Fresnel zone plates (FZP), ${ }^{12}$ or compound diffractive lenses, ${ }^{13}$ 2DWGs deliver nanoscale $\mathrm{x}$-ray beams with controllable spatial coherence. The quasi-point source of the WG exit emits smooth wavefronts which are ideally suited for holographic imaging. ${ }^{1}$ At the same time, the absorption in the cladding assures extremely low background illumination, without contributions from higher diffraction orders as in FZP focusing or the pronounced tails of KBoptics. Hence, they also enable nanoscale diffraction studies at significantly reduced background. ${ }^{14,15}$ In addition to spatial and coherence filtering, WGs can also concentrate the beam, based on geometric tapering. ${ }^{16,17}$ For present imaging applications, WGs without beam compression are used and have to be combined with high gain pre-focusing optics to reach the flux needed for imaging applications. ${ }^{7}$ In a state of the art realization of this scheme, a KB-beam of about 200-300 nm cross-section was coupled into WGs with an exit size of $d=50-70 \mathrm{~nm}$, delivering a flux in the range ${ }^{1}$ $10^{8}-10^{9} \mathrm{ph} / \mathrm{s}$. The smallest WG beam diameters down to $10 \mathrm{~nm}$ have been achieved ${ }^{18}$ by crossing two high transmission $1 D W G$ s slices to form an effective $2 \mathrm{DWG}$. This crossed 2DWG system is limited, however, for reasons of its intrinsic astigmatism, as well as the absorption in the carbon guiding layer of the WGs, which becomes important for lower photon energy. Furthermore, the planar thin film fabrication technology is not compatible with variations in shape function. To overcome these limits, we have developed an alternative fabrication scheme based on e-beam lithography, reactive ion etching, and wafer bonding, which gives buried air or vacuum channels in silicon. ${ }^{19}$
In this work, we use this fabrication approach to realize WGs with a taper function, which concentrate and filter the beam at the same time. This can either be used to match the entrance width $D$ to the pre-focusing optics and to funnel more photons to a smaller exit width $d$, or in future extensions to make pre-focusing optics in front of the WG obsolete, at least for some applications. An earlier experimental realization of a tapered waveguide $(\mathrm{tWG})^{16}$ was demonstrated by an air-gap $1 \mathrm{DWG},{ }^{20}$ while tapered $2 \mathrm{DWG}$ is addressed in this work. To demonstrate the concept of tWG beam compression in lithographically defined channels, we couple an undulator beam focused by KB-mirrors to $300 \mathrm{~nm}$ into a tWG and guide it further to $d \simeq 75 \mathrm{~nm}$ at the exit.

For the design of the present experiment, we have used finite difference simulations (FDSs) to study the beam propagation in tWGs, based on the parabolic wave equation. ${ }^{21}$ In addition, the propagation in $\mathrm{x}$-ray WGs with straight channels $^{20-22}$ has been studied previously, showing that beam compression and filtering can be combined in a tWG. ${ }^{23}$ Both Bergemann et al. ${ }^{24}$ and Kukhlevsky et al. ${ }^{25}$ have studied linearly tapered x-ray WGs or capillaries by analytical and numerical calculations, addressing the minimum beam width at the exit; while Panknin et al. ${ }^{23}$ have also included non-linear tapering profiles and Bukreeva et al. ${ }^{26}$ have studied corrugated WGs.

Fig. 1(a) shows the wave optical simulation of guided wave propagation in a one-dimensional linear tWG (vacuum in silicon), as obtained by FDSs. The WG length $L=5 \mathrm{~mm}$ was chosen for all WGs to ensure sufficient absorption of radiative modes at the given photon energy $E=13.8 \mathrm{keV}$, with a calculated transmission $T_{s i}=5.25 \times 10^{-7}$, correspondingly assuring an extremely low background signal. The slope of the WG interface and the associated linear tapering geometry are limited by the critical angle of total internal reflection $\theta_{c} \simeq \sqrt{2 \delta}=2.25 \mathrm{mrad}$ corresponding to the index of refraction $n_{s i}=1-\delta+i \beta$, with $\delta=2.554 \times 10^{-6}$ and $\beta=2.047 \times 10^{-8}$, and guiding layer with $n_{v a c}=1$. In the example shown in Fig. 1(a), the entrance and exit sizes of the WG are $D=334 \mathrm{~nm}$ and $d=73 \mathrm{~nm}$, and the taper angle $\theta=26 \mu \mathrm{rad}$. The simulation illustrates the rapid decay 


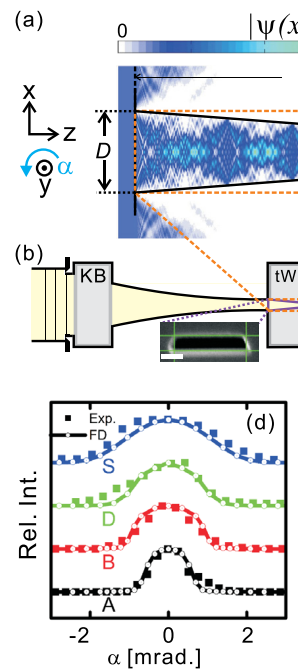

\section{$\left.\right|^{2} /\left|\psi_{0}\right|^{2}$}
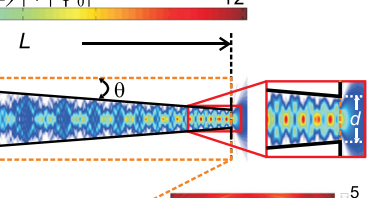

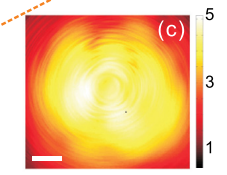

FIG. 1. (a) FDS of wave propagation in a WG channel (vacuum) in silicon with linear taper for $13.8 \mathrm{keV}$ photon energy. A plane wave is coupled into the front side of the WG with total length of $L=5 \mathrm{~mm}$ and entrance and exit width of $D=334 \mathrm{~nm}$ and $d=73 \mathrm{~nm}$, respectively, corresponding to a taper angle $\theta=26 \mu \mathrm{rad}$. The local intensity maximum in the exit plane is enhanced by a factor of 11 compared to the entrance plane, due to guided beam compression by the taper geometry. (b) Schematic of the experimental setup at the P10 coherence beamline of the storage ring PETRA III, DESY. The entrance side of the WG is placed in the focal plane of the KB-mirror. Scanning electron micrographs show the entrance and exit face of a tWG (labeled as "C" below). Scale bar: $100 \mathrm{~nm}$. (c) Far-field image of the tWG with flux of $2.69 \times 10^{8} \mathrm{cps}$ on logarithmic scale. Scale bar: $5 \mathrm{~mm}$. (d) Integrated far-field intensity as a function of WG rotation angle, $(\alpha)$, normalized to the maximum. (e) The angular acceptance $\omega$ determined from the central maxima of the curves shown in (d), as a function of $\theta$.

of the wave intensity in bulk silicon and the much weaker absorption in the tapered guiding layer. With $\psi(x, z)$ denoting the field inside the WG and $\psi_{0}$ the incident plane wave, the intensity enhancement can be defined as the maximum of the normalized intensity $I_{\max }=\max \left\{|\psi(x, z)|^{2} /\left|\psi_{0}\right|^{2}\right\}$, where $I_{\max }=11$ for the simulated WG in Fig. 1(a). This enhancement appears towards the end of the WG as a maximum with a flux density one order of magnitude higher than at the WG entrance. In this manner, beam compression by the tWG is clearly evidenced. In order to demonstrate this concept experimentally, we have fabricated a WG chip with many parallel tWGs, and finally diced to $L=5 \mathrm{~mm}$. ${ }^{19,27}$ The optical characterization of the WGs was performed at the GINIX (Göttingen Instrument for Nano-Imaging with X-rays) at the P10 beamline at the PETRA III synchrotron facility in Hamburg (DESY). Fig. 1(b) shows a schematic of the experimental setup, along with electron micrographs of the entrance and exit plane of a representative WG (labeled as "C" below). The WG entrance had a width of $334 \mathrm{~nm}$ and a depth of $69 \mathrm{~nm}$; the WG exit had a width of $73 \mathrm{~nm}$ and a depth of $51 \mathrm{~nm}$. The WG entrance was aligned in the focal plane of the KB-mirror system. The far-field images were recorded by a single photon counting pixel detector (Pilatus $300 \mathrm{~K}$, Dectris) with $172 \times 172 \mu \mathrm{m}^{2}$ pixel size, placed $5.1 \mathrm{~m}$ behind the WG. For $13.8 \mathrm{keV}$, a KB-flux of $I_{K B}=7.32 \times 10^{10}$ photons $/ \mathrm{s}$ was recorded, and a focal spot
TABLE I. Entrance and exit size of the guiding layer, with the corresponding taper angle $\theta$, and the angular acceptance $\omega$ for the tWGs labeled as "A," "B," "C," and "D" along with a straight reference channel "S."

\begin{tabular}{lccccc}
\hline \hline WG label & A & B & C & D & S \\
\hline$D(\mathrm{~nm})$ & $686 \times 67$ & $478 \times 65$ & $334 \times 69$ & $180 \times 70$ & $74 \times 70$ \\
$d(\mathrm{~nm})$ & $136 \times 63$ & $168 \times 65$ & $73 \times 51$ & $78 \times 50$ & $75 \times 50$ \\
$\theta / \theta_{c}$ & 0.0244 & 0.0137 & 0.0116 & 0.0045 & $\leq 4 \times 10^{-5}$ \\
$\omega / \theta_{c}$ & 0.511 & 0.675 & $\cdots$ & 0.875 & 1.187 \\
\hline \hline
\end{tabular}

size of $295 \mathrm{~nm}$ (FWHM) in horizontal and $181 \mathrm{~nm}$ (FWHM) in vertical direction, respectively. The far-field intensity distribution of the WG labeled "C" is shown in Fig. 1(c), with $2.79 \times 10^{10}$ photons/s impinging onto its entrance, for a $1 \mathrm{~s}$ accumulation. The flux at the exit was measured to $I_{W G}^{C}=$ $2.69 \times 10^{8}$ photons/s. Next, the angular acceptance $\omega$ of the WG, defined as the width (FWHM, Gaussian fit) of the exit intensity as a function of angle of incidence, was determined by rotating the WG around the $y$-axis by an angle $\alpha$ and measuring the integrated far-field intensity, as shown as solid squares in Fig. 1(d). In addition, numerical simulations for WGs of different taper angles $\theta$, labeled by A, B, and D with $\theta=55,31$, and $10.2 \mu \mathrm{rad}$, respectively, are depicted by lines. Label "S" stands for a straight channel reference. Both curves are normalized to the maximum intensity and are in very good agreement. As shown in Fig. 1(e), the angular acceptance decreases with the taper angle $\omega(\theta)$ as expected from wave optical simulations (Table I). In a geometric optical framework, this is explained by the increase in the number of reflections $n$ and in particular, by the correspondingly increasing internal reflection angles $\theta_{n}$ at the interfaces between guiding layer and cladding, see also the sketch in Fig. 3(b). The beam can only be guided in the $z$ direction, as long as the internal angle is smaller than the critical angle $\theta_{n} \leq \theta_{c}$, else it is absorbed in the cladding.

A precise analysis of the beam confinement due to the WG geometry is obtained by reconstructing the complex wave field using the measured far-field intensities in the nearfield regime right behind the WG exit using the error reduction (ER) algorithm. ${ }^{18}$ The reconstructed near-field intensity distribution and the corresponding reconstruction of the farfield pattern are shown in Fig. 2. As usual in iterative phase retrieval methods, the "reconstructed far-field" is computed from the reconstructed near-field intensity and can be tested against the measured far-field (see Fig. 1(c)), in order to check consistency and convergence of the phase retrieval process. The ER algorithm was initialized with the auto-correlation of the near-field, obtained by an inverse Fourier transform of the recorded far-field data, shown in Fig. 1(c). The support constraint was implemented using a Gaussian function with a width (FWHM) eight times larger than the size of the WG exit, in the respective direction. Fig. 2(a) shows the reconstructed far-field intensity pattern of a tWG ("C") on logarithmic scale, which is found in very good agreement with the measured data, as shown in Fig. 1(c). Figs. 2(b) and 2(c) show intensity and phase of the reconstructed near-field image in the WG exit plane. Gaussian fits to the central peak of the near-field intensity along the principal directions are shown in Figs. 2(d) and 2(e), respectively. They indicate an effective 

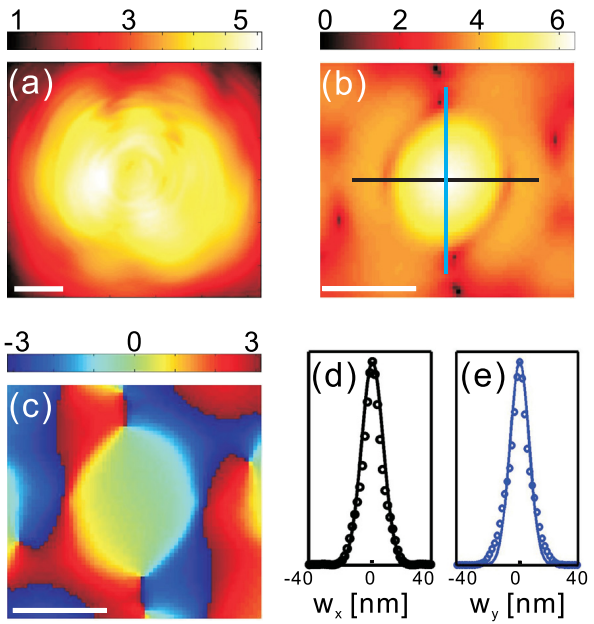

FIG. 2. Results of the reconstruction by the ER algorithm. (a) Reconstructed far-field image of the tWG (labeled as "C"), using logarithmic scale. Scale bar: $5 \mathrm{~mm}$. (b) Intensity (logarithmic scale) and (c) phase (in radian) of reconstructed near-field distribution, obtained after 1500 ER iterations. Scale bar: $40 \mathrm{~nm}$. (d) and (e) Gaussian fits to two perpendicular profiles of the central peak, along $x$ - and $y$-axis.

source with a width of $16.48 \mathrm{~nm}$ ( $x$-axis) and depth of $14.60 \mathrm{~nm}$ ( $y$-axis); the results for the tapered ("C $C$ ") and straight ("S") channels are also tabulated in Table II. The transmission of the WG defined as the ratio of the intensity impinging onto the WG and the intensity exiting the WG is calculated using

$$
T_{S i m}=\frac{\int_{-\frac{d}{2}}^{\frac{d}{2}}|\psi(x, z=L)|^{2} d x}{\int_{-\frac{D}{2}}^{\frac{D}{2}}|\psi(x, z=0)|^{2} d x} \quad \text { and } \quad T_{E x p}=\frac{I_{W G} \times A_{K B}}{I_{K B} \times A_{W G}},
$$

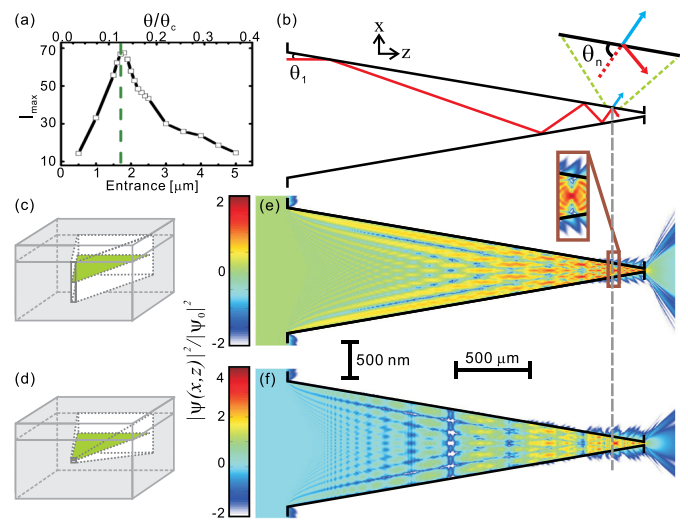

FIG. 3. FDS of a tWG in Si for $d=10 \mathrm{~nm}, L=2.4 \mathrm{~mm}$, and $E=11 \mathrm{keV}$ $\left(\theta_{c}=2.84 \mathrm{mrad}\right)$. (a) The curve $I_{\max }(\theta)$ has a maximum at $\theta_{\max }=0.124 \theta_{c}$. (b) For the given parameters, the channel supports 4 internal reflections, before leakage is observed at $\theta_{5}>\theta_{c}$. (c) and (d) Sketch of the linear taper geometry with taper in (c) one and (d) two dimensions. (e) and (f) Wave propagation inside a (e) one- and (f) two-dimensional tWG, with intensity plotted on logarithmic scale.
TABLE II. Comparison of a representative tWG channel "C" and a straight channel "S," selected for an identical exit aperture of about $70 \times 50 \mathrm{~nm}$, and recorded under identical settings of the KB-focused beam. The total WG intensity, the corresponding experimental transmission value $T_{E x p}$, along with the numerically predicted values $T_{S i m}$, and beam spot size in the WG exit plane, $\mathrm{w}_{x / y}(\mathrm{FWHM})$ are shown.

\begin{tabular}{lccccc}
\hline \hline WG label & $I_{W G}(\mathrm{cps})$ & $T_{E x p}(\%)$ & $T_{\text {Sim }}(\%)$ & $\mathrm{w}_{x}(\mathrm{~nm})$ & $\mathrm{w}_{y}(\mathrm{~nm})$ \\
\hline $\mathrm{C}$ & $2.69 \times 10^{8}$ & 0.96 & 8.9 & 16.48 & 14.60 \\
$\mathrm{~S}$ & $3.34 \times 10^{7}$ & 0.47 & 5.9 & 16.95 & 14.13 \\
\hline \hline
\end{tabular}

for the simulation $T_{S i m}$ and the experiment $T_{E x p}$, where $A_{W G}$ and $A_{K B}$ are the cross section areas of the WG entrance and the KB beam size. The measured flux of the tWG (" $\mathrm{C}$ ") is $I_{W G}^{C}=2.69 \times 10^{8} \mathrm{cps}$ and of the straight reference WG ("S") $I_{W G}^{S}=3.34 \times 10^{7} \mathrm{cps}$. The simulated transmission $T_{\text {Sim }}=8.9 \%$ of the tWG is 9.27 times higher than the experimental transmission $T_{E x p}=0.96 \%$, which is affected by channel roughness and internal defects. The gain $G$, defined as the ratio of the intensity of the tWG over the straight reference, is calculated by

$$
G_{S i m}=\frac{\int_{t W G}|\psi(x, z=L)|^{2} d x}{\int_{W G}|\psi(x, z=L)|^{2} d x} \quad \text { and } \quad G_{E x p}=\frac{I_{W G}^{C}}{I_{W G}^{S}},
$$

yielding to $G_{\text {Sim }}=8.05$ for the numerical simulation, and $G_{E x p}=6.89$ for the experiment. This demonstrates clearly that a linear tWG can efficiently compress the beam, even if real structure effects such as roughness and waviness deteriorate the experimental values for $T$. In fact, after obtaining the results shown in Figs. 1 and 2, further improvements in the fabrication protocol regarding, in particular, wafer bonding under inert gas and changes in the exposure protocol were implemented and led to significantly better controlled channel side walls, and correspondingly higher $T$ as detailed in elsewhere. $^{27}$

In order to further optimize tWGs in view of $I_{\max }$, the entrance width can be enlarged to collect more photons from the incoming beam, while the exit width can be tailored to the favored size and divergence for a specific experiment. In this way, the improved WG imaging could even be implemented without high gain focusing optics, such as the present KB-system. Fig. 3(a) illustrates the intensity enhancement $I_{\max }$ as a function of the taper angle $\theta$, achieved by compressing a beam to an exit size of $d=10 \mathrm{~nm}$ over a channel length $L=2.4 \mathrm{~mm}$, simulated for vacuum in $\mathrm{Si}$ at $E=11 \mathrm{keV}$. We chose $d=10 \mathrm{~nm}$ in view of holographic imaging experiments which require a small source size. The $I_{\max }(\theta)$ curve first steeply increases, as more photons are collected, but reaches a maximum at $\theta_{\max }=0.124 \theta_{c}$, before declining again, when the internal reflection angle increases above the critical angle $\theta_{n} \geq \theta_{c}$ towards the end of the channel, see (b). For illustration, we trace a ray for $\theta_{\max }$ shown as a red line in (b). From $\theta_{n}=(2 n-1) \theta \leq \theta_{c}$, we can infer that the channel supports $n=4$ internal reflections. ${ }^{27}$ For higher numbers of reflections $n \geq 5, \theta_{n} \geq \theta_{c}$ lead to a leaking into the cladding material, see blue arrow in (b). However, a fraction of the x-ray beam is still reflected and guided inside the tWG (red arrow). The 
gray dashed line in (b), (e), and (f) indicates the position where the fifth reflection occurs, corresponding to a tWG beam with width of $16.34 \mathrm{~nm}$. This is in good agreement with the visual inspection of the leakage based on the logarithmically scaled intensity distribution, see (e) and (f). Despite the fact that the reflected angle exceeds the critical angle towards the end of the channel, the WG still reaches $I_{\max }=66.7$, underlining the substantial compression. For the same parameters, but in a two-dimensional taper geometry, the simulation yields $I_{\max }=4206$, as shown in (f).

In summary, tWGs have been designed based on FDSs, fabricated and characterized by experiments with synchrotron radiation. A pre-focused monochromatic x-ray beam was coupled into the front side of a tWG with an entrance width matching the focal spot size. The beam coupled into the WG was further compressed by propagation in the guiding channel with linear taper, resulting in a gain of about one order of magnitude over a straight channel waveguide. Simulations for a two-dimensional cone geometry indicate that gain values $G \simeq 10^{4}$ could be reached by a WG tapered in two dimensions, given future improvements in realizing tapered profiles in both directions orthogonal to the optical axis.

We thank Mike Kanbach for helping WG fabrication, Anna-Lena Robisch for phase retrieval, and Michael Sprung for the support at the P10 beamline. We gratefully acknowledge the German Research Foundation (DFG) for funding through Grant No. SFB $755 / \mathrm{C} 1$, and appreciate the financial support from the Ministry of Science and Technology of China (NSC 102-2917-I-564-062).

${ }^{1}$ M. Bartels, M. Krenkel, J. Haber, R. Wilke, and T. Salditt, Phys. Rev. Lett. 114, 048103 (2015).

${ }^{2}$ E. Spiller and A. Segmüller, Appl. Phys. Lett. 24, 60 (1974).

${ }^{3}$ Y. P. Feng, S. K. Sinha, H. W. Deckman, J. B. Hastings, and D. P. Siddons, Phys. Rev. Lett. 71, 537 (1993).

${ }^{4}$ S. Di Fonzo, W. Jark, S. Lagomarsino, C. Giannini, L. De Caro, A. Cedola, and M. Müller, Nature 403, 638 (2000).
${ }^{5}$ W. Jark, A. Cedola, S. Di Fonzo, M. Fiordelisi, S. Lagomarsino, N. V. Kovalenko, and V. A. Chernov, Appl. Phys. Lett. 78, 1192 (2001).

${ }^{6}$ F. Pfeiffer, C. David, M. Burghammer, C. Riekel, and T. Salditt, Science 297, 230 (2002).

${ }^{7}$ A. Jarre, C. Fuhse, C. Ollinger, J. Seeger, R. Tucoulou, and T. Salditt, Phys. Rev. Lett. 94, 074801 (2005).

${ }^{8}$ P. Kirkpatrick and A. V. Baez, J. Opt. Soc. Am. 38, 766 (1948).

${ }^{9}$ O. Hignette, P. Cloetens, W.-K. Lee, W. Ludwig, and G. Rostaing, J. Phys. IV France 104, 231 (2003).

${ }^{10}$ H. Mimura, S. Matsuyama, H. Yumoto, H. Hara, K. Yamamura, Y. Sano, M. Shibahara, K. Endo, Y. Mori, Y. Nishino et al., Jpn. J. Appl. Phys., Part 2 44, L539 (2005).

${ }^{11}$ S. Matsuyama, H. Mimura, H. Yumoto, Y. Sano, K. Yamamura, M. Yabashi, Y. Nishino, K. Tamasaku, T. Ishikawa, and K. Yamauchi, Rev. Sci. Instrum. 77, 103102 (2006).

${ }^{12}$ W. Chao, B. D. Harteneck, J. A. Liddle, E. H. Anderson, and D. T. Attwood, Nature 435, 1210 (2005).

${ }^{13}$ C. G. Schroer and B. Lengeler, Phys. Rev. Lett. 94, 054802 (2005).

${ }^{14}$ M. Müller, M. Burghammer, D. Flot, C. Riekel, C. Morawe, B. Murphy, and A. Cedola, J. Appl. Cryst. 33, 1231 (2000).

${ }^{15}$ C. Krywka, H. Neubauer, M. Priebe, T. Salditt, J. Keckes, A. Buffet, S. V. Roth, R. Doehrmann, and M. Müller, J. Appl. Cryst. 45, 85 (2012).

${ }^{16}$ M. J. Zwanenburg, J. H. H. Bongaerts, J. F. Peters, D. Riese, and J. F. van der Veen, Physica B 283, 285 (2000).

${ }^{17}$ I. Bukreeva, D. Pelliccia, A. Cedola, F. Scarinci, M. Ilie, C. Giannini, L. De Caro, and S. Lagomarsino, J. Synchrotron Radiat. 17, 61 (2010).

${ }^{18}$ S. P. Krüger, H. Neubauer, M. Bartels, S. Kalbfleisch, K. Giewekemeyer, P. J. Wilbrandt, M. Sprung, and T. Salditt, J. Synchrotron Radiat. 19, 227 (2012).

${ }^{19}$ H. Neubauer, S. Hoffmann, M. Kanbach, K. Giewekemeyer, S. Kalbfleisch, S. P. Krüger, and T. Salditt, J. Appl. Phys. 115, 214305 (2014).

${ }^{20}$ M. J. Zwanenburg, J. F. Peters, J. H. H. Bongaerts, S. A. de Vries, D. L. Abernathy, and J. F. van der Veen, Phys. Rev. Lett. 82, 1696 (1999).

${ }^{21}$ C. Fuhse and T. Salditt, Appl. Opt. 45, 4603 (2006).

${ }^{22}$ L. De Caro, C. Giannini, S. Di Fonzo, W. Yark, A. Cedola, and S. Lagomarsino, Opt. Commun. 217, 31 (2003).

${ }^{23}$ S. Panknin, A. Hartmann, and T. Salditt, Opt. Commun. 281, 2779 (2008).

${ }^{24}$ C. Bergemann, H. Keymeulen, and J. F. van der Veen, Phys. Rev. Lett. 91, 204801 (2003).

${ }^{25}$ S. V. Kukhlevsky, F. Flora, A. Marinai, G. Nyitray, Zs. Kozma, A. Ritucci, L. Palladino, A. Reale, and G. Tomassetti, X-Ray Spectrom. 29, 354 (2000).

${ }^{26}$ I. Bukreeva, A. Popov, D. Pelliccia, A. Cedola, S. B. Dabagov, and S. Lagomarsino, Phys. Rev. Lett. 97, 184801 (2006).

${ }^{27}$ See supplementary material at http://dx.doi.org/10.1063/1.4921095 for details about fabrication and characterization of tapered X-ray waveguides. 



\section{Chapter 5}

\section{Supplemental Material}

Reprinted from

H.-Y. Chen, S. Hoffmann and T. Salditt

Supplemental Material for X-ray beam compression by tapered waveguides

Applied Physics Letters, 106(19), 194105 (2015)

with the permission of AIP Publishing. 


\title{
X-Ray Beam Compression by Tapered Waveguides \\ APL-Supplementary Material
}

\author{
H.-Y. Chen(陳心誼), S. Hoffmann and T. Salditt \\ hchen2@gwdg.de, tsaldit@gwdg.de
}

Institut für Röntgenphysik, Universität Göttingen, Friedrich-Hund-Platz 1, 37077 Göttingen, Germany

\section{FABRICATION}

For a waveguide (WG) chip with air-filled channels, the employed cladding layer is Si and guiding layer is air. The fabrication process is presented schematically in Fig. S1. One-side polished 4 inch (100) Si-Wafers (University Wafer) with thickness of $1000 \pm 25 \mu \mathrm{m}$ have been used as a substrate. A layer of a poly-methyl-methacrylate photoresist (A2 by MicroChem) was spin-coated onto a silicon wafer at 1000rpm for 70s resulting in a thickness of about $400 \mathrm{~nm}$ and subsequently structured by electron beam lithography using an electron dose of $600 \mu \mathrm{C} / \mathrm{cm}^{2}$. For the development the illuminated sample was immersed into a 1:3 compound of methylisobutylketone (MIBK) and isopropanol (IPA) at $4^{\circ} \mathrm{C}$ for 30 s and afterwards dipped into IPA for another 30s to stop the development process before rinsing the sample with nitrogen gas. The photoresist acts as an etching mask while transferring the structures into the silicon wafer via reactive ion etching (RIE). Thereby a gas flow of $15 \mathrm{sccm}$ : $35 \mathrm{sccm}$ of $\mathrm{SF}_{6}$ and $\mathrm{CHF}_{3}$ for etching and sidewall passivation was employed at a power of $100 \mathrm{~W}$ for 50s (Plasmalab System 100, Oxford Instruments Plasma Technologies). Next, the mask was removed by cleaning in an RCA bath consisting of an 5:1:1 aqueous solution of deionized $\mathrm{H}_{2} \mathrm{O}, \mathrm{NH}_{4} \mathrm{OH}$ and $\mathrm{H}_{2} \mathrm{O}_{2}$ at $65^{\circ} \mathrm{C}$ for $15 \mathrm{~min}$. The scanning electron micrographs in Fig. S1(b) show the quality of the etched structure and that the desired taper-shape can be achieved. The following steps are hydrophilic wafer bonding under cleanroom conditions and annealing at $750^{\circ} \mathrm{C}$ for $2 \mathrm{~h}$ in an air atmosphere. A detailed description of the fabrication steps and parameters for WG chips can also be found in Neubauer et al. (2014). The sample was finally cut to a length $L$ depending on the requirements of the experiment, in particular the photon energy. For the results reported here, a length of $L=5 \mathrm{~mm}$ for a photon energy of $13.8 \mathrm{keV}$ was chosen. The cross-sections after dicing, meaning the scanning electron micrographs for the entrance and exit side of a WG, are shown in Figs. S1(c) and S1(d) for a tapered waveguide (tWG) and a $2 \mathrm{DWG}$, respectively. The entrance and exit size are $334 \mathrm{~nm}$ (width) 
$\times 69 \mathrm{~nm}($ depth $)$ and 73nm (width) $\times 51 \mathrm{~nm}($ depth $)$ for the $\mathrm{tWG}$, and 74nm (width) $\times 70 \mathrm{~nm}$ (depth) and $75 \mathrm{~nm}($ width $) \times 50 \mathrm{~nm}($ depth) for the straight one.
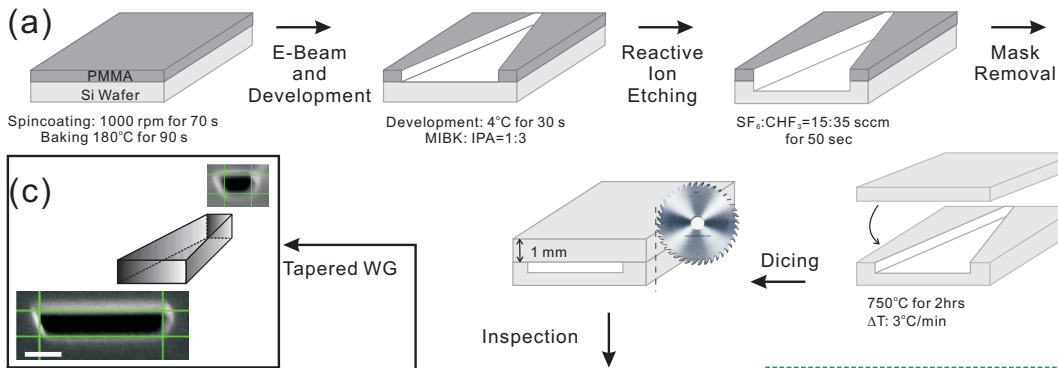

(d)
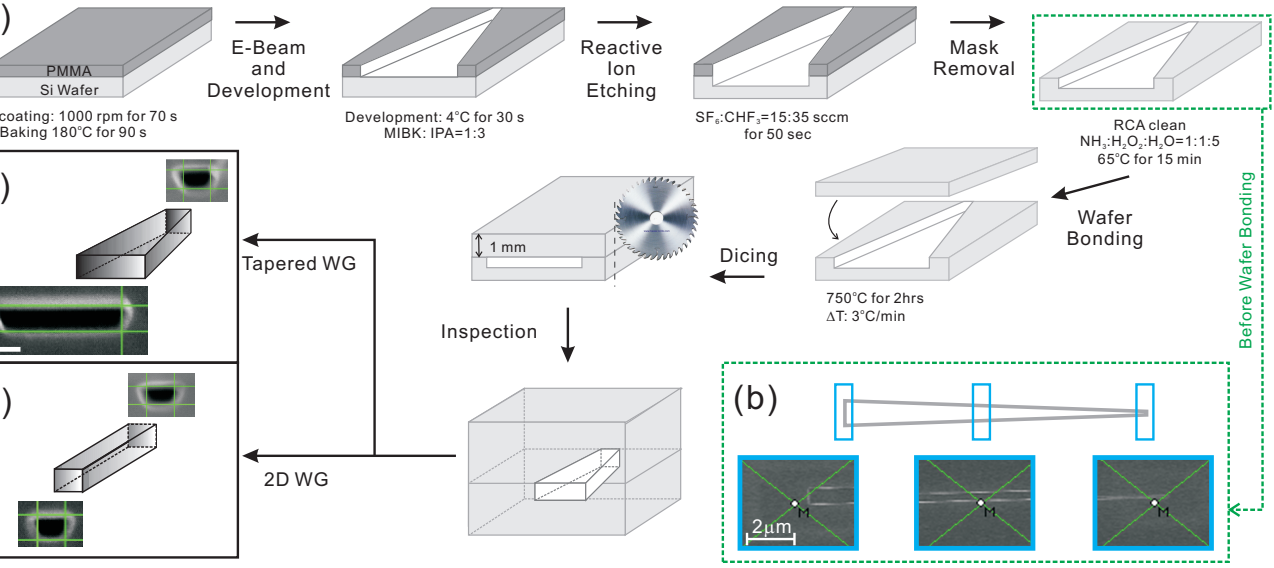

Figure S 1: (a) Fabrication steps for an air-filled tWG, involving e-beam lithography and development, pattern transfer by reactive ion etching, capping by wafer bonding, and dicing. (b) Scanning electron micrographs exhibit the taper-shape after etching process. Also scanning electron micrographs of the entrance and exit side of (c) the tapered and (d) the 2D WG after dicing are shown. Scale bars: $100 \mathrm{~nm}$.

\section{CHARACTERIZATION}

The fabricated structure consists of orientation WGs (one-dimensional planar WGs) for facilitating the alignment, labeled as 'O', straight channel WGs (two-dimensional straight channel WGs) labeled as 'S', and several groups of tWGs. The entrance widths of the tWGs range from $600 \mathrm{~nm}$ down to $150 \mathrm{~nm}$, labeled as A, B, C, and D. The exit widths of the tWGs ranges from $200 \mathrm{~nm}$ down to $30 \mathrm{~nm}$, written as subscripts (1-8). For 2DWGs, the width of the straight channel varies in the same manner as the exit size of the tWGs. All details are shown in Tab. SI. 
(a) Entrance width $[\mathrm{nm}]$

\begin{tabular}{l|l|l|l|l}
\hline \hline Group label & A & B & C & D \\
\hline Entrance width & 600 & 450 & 300 & 150 \\
\hline \hline
\end{tabular}

(b) Exit width $[\mathrm{nm}]$

\begin{tabular}{l|l|l|l|l}
\hline \hline Group label & $1-2$ & $3-4$ & $5-6$ & $7-8$ \\
\hline A,B,C & 200 & 100 & 60 & 30 \\
\hline Exit width & 100 & 60 & 30 & - \\
\hline \hline
\end{tabular}

(c) Channel Width

\begin{tabular}{l|l|l|l|l|l|l|l|l}
\hline WGs number & 1 & 2 & 3 & 4 & 5 & 6 & 7 & 8 \\
\hline $\mathrm{S}[\mathrm{nm}]$ & 200 & 200 & 100 & 100 & 60 & 60 & 30 & 30 \\
\hline $\mathrm{O}[\mu \mathrm{m}]$ & 50 & 30 & 10 & 1 & 1 & 5 & 30 & 30 \\
\hline \hline
\end{tabular}

(d) Taper angle $\theta[\mu \mathrm{rad}]$

\begin{tabular}{l|l|l|l|l}
\hline WGs number & $1-2$ & $3-4$ & $5-6$ & $7-8$ \\
\hline $\mathrm{A}$ & 40 & 50 & 0.054 & 57 \\
\hline $\mathrm{B}$ & 25 & 35 & 39 & 42 \\
\hline $\mathrm{C}$ & 10 & 20 & 24 & 27 \\
\hline $\mathrm{D}$ & 5 & 9 & 12 & - \\
\hline $\mathrm{S}, \mathrm{O}$ & 0 & 0 & 0 & 0 \\
\hline \hline
\end{tabular}

Table S I: Design parameters: (a) The entrance widths of the tWGs range from $600 \mathrm{~nm}$ down to $150 \mathrm{~nm}$. (b) The exit widths of the tWGs range from $200 \mathrm{~nm}$ down to $30 \mathrm{~nm}$. (c) The width of the orientation and straight channel varies in the same manner as the exit width of the tWGs. (d) The taper angles $\theta$ of the WGs. 

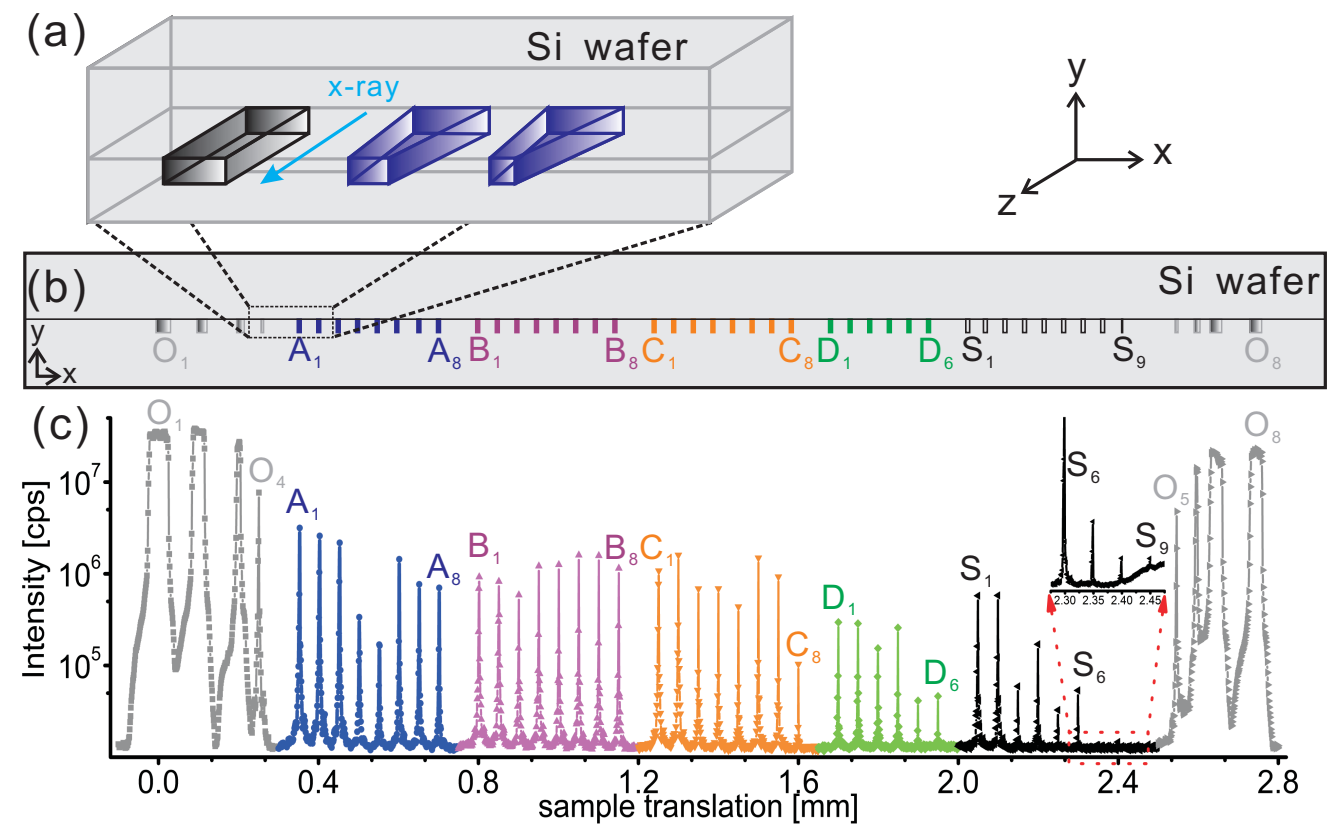

Figure S 2: (a) The schematic of a WG chip with (b) schematic cross section at exit side. (c) The aligned structure is translated through the focus of the KB-mirror system to locate the positions of the individual WG channels. The WG exit flux is plotted in logarithmic scale.
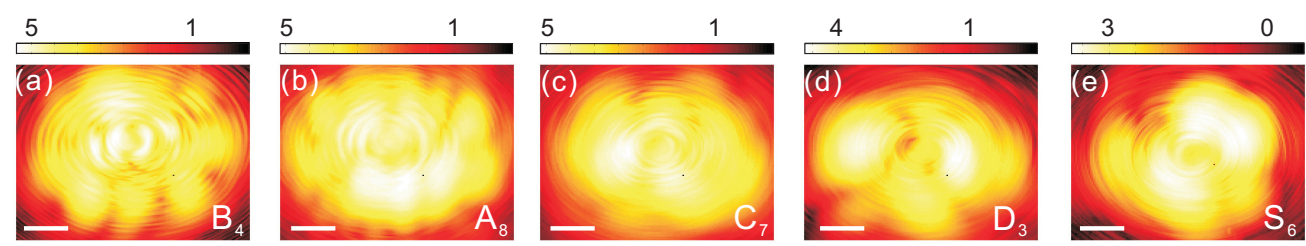

Figure S 3: Far-field intensities depending on the exit size of the WGs, using a WG chip of a length $L=5 \mathrm{~mm}$. The intensity (logarithmic plot) was recorded within 1 second. Detector: Pilatus 300K, ROI: $170 \mathrm{px} \times 170 \mathrm{px}$. Scale bars: $5 \mathrm{~mm}$.

The measurements were performed at the Nano-focus endstation EH2 (the Göttingen Instrument for Nano-Imaging with X-rays, GINIX) of the P10 beamline at the PETRA III synchrotron facility in Hamburg (DESY). The beam was prefocused by KB-mirror. The WG chip was placed into the focal plane of the KB-mirror. The sample holder system offers three translational degrees of freedom $x$ (perpendicular to the propagation direction), $y$ 


\begin{tabular}{l|c|c|c|c|c}
\hline \hline full WGs label & $\mathrm{B}_{4}$ & $\mathrm{~A}_{8}$ & $\mathrm{C}_{7}$ & $\mathrm{D}_{3}$ & $\mathrm{~S}_{6}$ \\
\hline WGs label (manuscript) & $\mathrm{B}$ & $\mathrm{A}$ & $\mathrm{C}$ & $\mathrm{D}$ & $\mathrm{S}$ \\
\hline$D(\mathrm{~nm})[\mathrm{H} \times \mathrm{V}]$ & $478 \times 65$ & $686 \times 67$ & $334 \times 69$ & $180 \times 70$ & $74 \times 70$ \\
\hline$d(\mathrm{~nm})[\mathrm{H} \times \mathrm{V}]$ & $168 \times 65$ & $136 \times 63$ & $73 \times 51$ & $78 \times 50$ & $75 \times 50$ \\
\hline$\theta(\mu \mathrm{rad})$ & 31 & 55 & 26.1 & 10.2 & 0.1 \\
\hline$I_{W G}(\mathrm{cps})$ & $4.11 \times 10^{8}$ & $4.08 \times 10^{8}$ & $2.69 \times 10^{8}$ & $8.49 \times 10^{7}$ & $3.34 \times 10^{7}$ \\
\hline$T_{E x p}(\%)$ & 1.56 & 1.51 & 0.96 & 0.49 & 0.47 \\
\hline \hline
\end{tabular}

Table S II: It shows the performance of WGs with different geometry. $\theta, I_{W G}$, and $T_{E x p}$ are taper angle, integrated intensity, and transmission rate for WGs, respectively.

(perpendicular to the propagation direction) and $z$ (parallel to the propagation direction) as well as three rotational degrees of freedom around the $x$-, $y$-, and $z$-axis. The far-field images were recorded using a CCD detector (Pilatus $300 \mathrm{~K}, 172 \times 172 \mu \mathrm{m}^{2}$ pixel size) placed $5.1 \mathrm{~m}$ behind the WG chip. The flux of the far-field image within the KB-mirror is approximately $I_{0}=7.32 \times 10^{10} \mathrm{ph} / \mathrm{s}$. The focal spot size of the KB was both about $295 \mathrm{~nm}(\mathrm{FWHM})$ in horizontal and 181nm (FWHM) in vertical directions, measured by scanning the WG across the beam in $x$ - and $y$ - direction.

Figure S2(a) shows the schematic of a WG chip with one orientation WG and two tWGs. The arrangement of the imbedded WGs with different design parameters on the silicon wafer are shown in Fig. S2(b). The aligned structure is translated through the focus of the KBmirror system to locate the positions of the individual WG channels. The corresponding transmitted intensities of these WGs are shown in Fig. S2(c). The spacing between the tWGs is $50 \mu \mathrm{m}$ which is much larger than the focal beam size. According to these results, all WGs are able to transmit the incoming x-ray beam. This demonstrates that the fabrication process is highly reliable.

Figure S3 shows far-field images of WGs with different entrance widths but comparable channel depths at exit size. The size of the WGs, WG labels, and corresponding flux $\left(I_{W G}\right)$ and transmission rates $\left(T_{E X P .}\right)$ are shown in Tab. SII. Regarding the reduction of the channel width and comparable channel depth at the exit position, an obvious change can be observed in the far-field images. For the WG with the broadest lateral exit size (tWG: $\mathrm{B}_{4}$ ), vertical stripes can be observed at the center of the far-field diffraction pattern (see Fig. S3(a)). 


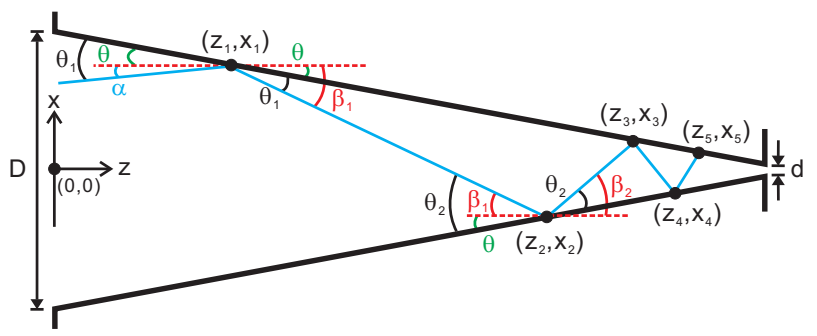

Figure S 4: Ray tracing inside a tWG.

The recorded patterns exhibit a characteristic far-field image depending on the number of guided modes, respectively the channel size. The far-field diffraction images of the WGs with similar exit size, shown in Figs. S3(c)-(e) are more homogeneous compared to the other two far-field patterns (see Figs. S3(a)-(b)). Regarding the exit flux and exit size, the WG $\mathrm{C}_{7}$ is well suited for x-ray imaging experiments.

\section{MULTIPLE REFLECTION IN A TAPERED WAVEGUIDE}

A ray tracing inside a tWG is shown in Fig. S4. The incident angle, tapered angle, $n_{t h}$ reflected angle, and the angle between incident beam and $z$-axis are $\alpha, \theta, \theta_{n}$, and $\beta_{n}$, respectively.

$$
\begin{array}{ll}
\theta_{1}=\theta+\alpha & \beta_{1}=2 \theta+\alpha \\
\theta_{2}=3 \theta+\alpha & \beta_{2}=4 \theta+\alpha \\
\theta_{3}=5 \theta+\alpha & \beta_{3}=6 \theta+\alpha \\
\vdots & \vdots \\
\theta_{n}=(2 n-1) \theta+\alpha & \beta_{n}=2 n \theta+\alpha
\end{array}
$$

The geometrical relations of $n_{t h}$ reflected angle $\theta_{n}$ and $\beta_{n}$ are given by $\alpha$ and $\theta$. The coordinate of $n_{t h}$ reflection $\left(z_{n}, x_{n}\right)$ can be calculated by the equations below.

$$
\begin{array}{ll}
z_{1}=\frac{D+z_{1}\left(\tan \beta_{1}-\tan \theta\right)}{\left(\tan \beta_{1}+\tan \theta\right)} & x_{1}=(-1)^{1-1}\left(\frac{D}{2}-z_{1} \tan \theta\right) \\
z_{2}=\frac{D+z_{2}\left(\tan \beta_{2}-\tan \theta\right)}{\left(\tan \beta_{2}+\tan \theta\right)} & x_{2}=(-1)^{2-1}\left(\frac{D}{2}-z_{2} \tan \theta\right) \\
z_{3}=\frac{x_{3}}{}=(-1)^{3-1}\left(\frac{D}{2}-z_{3} \tan \theta\right) \\
\vdots \\
z_{n}=\frac{D+z_{n-1}\left(\tan \beta_{\mathrm{n}-1}-\tan \theta\right)}{\left(\tan \beta_{\mathrm{n}-1}+\tan \theta\right)} & x_{n}=(-1)^{n-1}\left(\frac{D}{2}-z_{n} \tan \theta\right)
\end{array}
$$



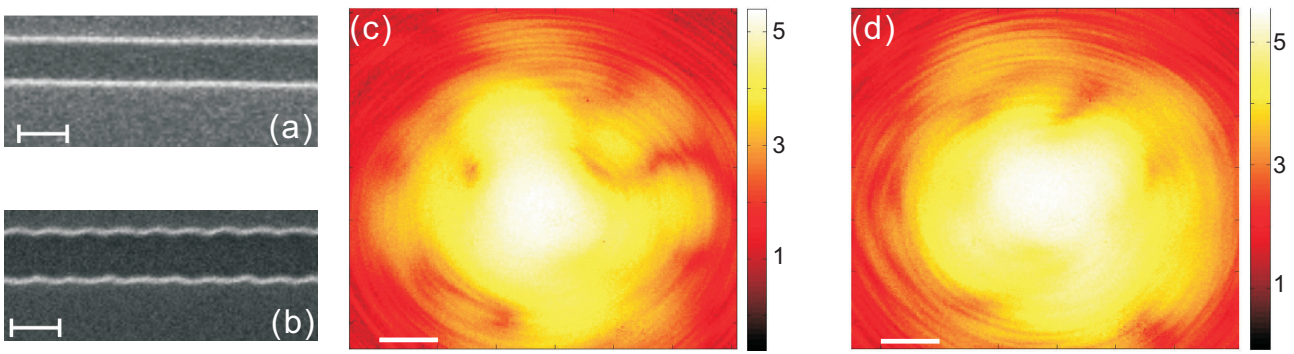

Figure S 5: SEM pictures before wafer bonding: (a) NEW protocol and (b) OLD protocol. Scale bars: 100nm. Far-field images of WGs with (a) straight channel and (d) tapered channel using NEW fabrication protocol. Scale bars: $2 \mathrm{~mm}$.

\section{FURTHER IMPROVEMENTS IN THE FABRICATION PROTOCOL YIELDING HIGH TRANSMISSION}

In fact, after obtaining the results shown in Figs. 1 and 2 of the manuscript, further improvements in the fabrication protocol regarding in particular wafer bonding under inert gas atmosphere and changes in the exposure protocol were implemented. The channel boundaries in Fig. S5(a) (NEW protocol) are of better quality than in Fig. S5(b) (OLD protocol), which shows a wavy contour. The parameters changed in the fabrication protocols are listed in Tab. SIII. Along with the better controlled channel shape, a significantly higher transmission $T$ was measured, see Tab. SIV and Figs. S5(c,d) for the measured far-field pattern of a (c) straight and (d) a tapered channel fabricated by the NEW protocol. Note that due to shutdown of PETRA III, a different synchrotron setup was used for these additional measurements, namely the upgraded ID01 beamline at ESRF (Grenoble, France), using the $E=8 \mathrm{keV}$ undulator beam, focused into the entrance of the channels by a Fresnel zone plate (FZP). The focal spot size was $305 \mathrm{~nm} \times 162 \mathrm{~nm}(\mathrm{H} \times \mathrm{V}, \mathrm{FWHM}$ values $)$, with a total intensity of $I_{0} \simeq 8.84-8.66 \times 10^{9} \mathrm{ph} / \mathrm{s}$, depending on the alignment conditions and ring current. The far-field diffraction patterns were recorded at high resolution with a pixel detector (Maxipix,ESRF) of $55 \mu \mathrm{m}$ pixel size, mounted on a diffractometer at a distance of $750 \mathrm{~mm}$. 


\begin{tabular}{c|c|c|c|c|c|c}
\hline \hline SEM label & protocol & photoresist & spin-coating & etching time & wafer bonding & $T_{\text {Exp }}(\%)$ \\
\hline Fig. S5(a) & NEW & 679.04 (Allresist) & $2000 \mathrm{rpm}$ for $70 \mathrm{sec}$ & $160 \mathrm{sec}$ & $1000^{\circ} \mathrm{C}$ for $4 \mathrm{~h}$ & $20 \sim 40$ \\
\hline Fig. S5(b) & OLD & A2(MicroChem) & $1000 \mathrm{rpm}$ for $70 \mathrm{sec}$ & $50 \mathrm{sec}$ & $750^{\circ} \mathrm{C}$ for $2 \mathrm{~h}$ & $0.5 \sim 2$ \\
\hline \hline
\end{tabular}

Table S III: Changes in the fabrication protocol, leading to significantly improved transmission. In addition, in the NEW protocol the annealing of the wafer bonding was carried out in inert gas $\left(\mathrm{N}_{2}\right.$ at a pressure of 300mbar, Nabertherm, P330), replacing air used in the OLD protocol.

\begin{tabular}{l|c|c|c|c|c}
\hline \hline Far-field images & type & $D(\mathrm{~nm})[\mathrm{H} \times \mathrm{V}]$ & $d(\mathrm{~nm})[\mathrm{H} \times \mathrm{V}]$ & $I_{W G}(\mathrm{cps})$ & $T_{E x p}(\%)$ \\
\hline Figure S5(c) & straight channel & $109 \times 85$ & $96 \times 89$ & $7.2 \times 10^{8}$ & 43.95 \\
\hline Figure S5(d) & tapered channel & $279 \times 125$ & $96 \times 83$ & $1.5 \times 10^{9}$ & 23.81 \\
\hline \hline
\end{tabular}

Table S IV: It shows the information and performance of WGs with different geometry. The flux of the incident beam is approximately $I_{0} \simeq 8.84-8.66 \times 10^{9} \mathrm{ph} / \mathrm{s}$. The beam size was both about $305 \mathrm{~nm}(\mathrm{FWHM})$ in horizontal and $162 \mathrm{~nm}(\mathrm{FWHM})$ in vertical direction.

[1] H. Neubauer, S. Hoffmann, M. Kanbach, K. Giewekemeyer, S. Kalbfleisch, S.P. Krüger, and T. Salditt, "High aspect ratio x-ray waveguide channels fabricated by e-beam lithography and wafer bonding," J. Appl. Phys. 115, 214305 (2014). 



\section{Chapter 6}

\section{Miniaturized beamsplitters realized by $x$-ray waveguides}

Reprinted from

S. Hoffmann-Urlaub and T. Salditt

Miniaturized beamsplitters realized by $x$-ray waveguides

Acta Crystallographica Section A, 72, 515-522 (2016)

with permission of the

International Union of Crystallography. 
FOUNDATIONS

ADVANCES

ISSN 2053-2733

Received 27 April 2016

Accepted 14 July 2016

Edited by J. Miao, University of California, Los Angeles, USA

Keywords: X-ray waveguides; X-ray interferometry; phase retrieval; coherence; coherent imaging.

\section{Miniaturized beamsplitters realized by X-ray waveguides}

\author{
Sarah Hoffmann-Urlaub* and Tim Salditt \\ Institut für Röntgenphysik, Universität Göttingen, Friedrich-Hund Platz 1, 37077 Göttingen, Germany. *Correspondence \\ e-mail: shoffma3@gwdg.de
}

This paper reports on the fabrication and characterization of X-ray waveguide beamsplitters. The waveguide channels were manufactured by electron-beam lithography, reactive ion etching and wafer bonding techniques, with an empty (air) channel forming the guiding layer and silicon the cladding material. A focused synchrotron beam is efficiently coupled into the input channel. The beam is guided and split into two channels with a controlled (and tunable) distance at the exit of the waveguide chip. After free-space propagation and diffraction broadening, the two beams interfere and form a double-slit interference pattern in the far-field. From the recorded far-field, the near-field was reconstructed by a phase retrieval algorithm (error reduction), which was found to be extremely reliable for the two-channel setting. By numerical propagation methods, the reconstructed field was then propagated along the optical axis, to investigate the formation of the interference pattern from the two overlapping beams. Interestingly, phase vortices were observed and analysed.

\section{Introduction}

In the visible range, waveguides are of paramount importance for different optical manipulations, such as filtering, confining, guiding, coupling or splitting of beams. In contrast, for X-rays, waveguide optics are much less developed. X-ray waveguide development started with demonstrations of propagation in planar (straight) waveguide structures fabricated by thin film deposition (Spiller, 1974; Feng et al., 1995; Lagomarsino et al., 1996; Zwanenburg et al., 1999; Pfeiffer et al., 2000; Prudnikov, 2003; Bongaerts et al., 2001; Pelliccia et al., 2005), followed by the development of two-dimensional channel waveguides (Pfeiffer et al., 2002; Jarre et al., 2005), which has posed tremendous challenges in fabrication. However, recent progress in lithography and wafer bonding (Neubauer et al., 2014; Chen et al., 2015; Bartels et al., 2015), in combination with numerical simulation and experimental setups, has helped to overcome long-standing restrictions. Presently, advanced X-ray waveguides will begin to provide much more functionalities than filtering the mode structure of a synchrotron beam (Osterhoff \& Salditt, 2011), such as beam concentration by tapering (Chen et al., 2015) or guiding beams around a bend (Salditt et al., 2015).

In this work, we introduce an important new functionality using X-ray waveguides, namely that of a beamsplitter. Simulations and experiments show that efficient splitting and guiding to selected exit distances are possible, opening up a new form of nano-interferometer. Previous work has already stressed the advantages of miniaturized X-ray interferometers for phase measurements at the nanoscale (Snigirev et al., 2009), extending the classical Bonse-Hart interferometer concept (Bonse \& Hart, 1965). In Snigirev et al. (2009), X-ray 


\section{research papers}

interferometers were realized based on a silicon bilens, i.e. by a pair of compound refractive lenses (CRL) etched into silicon. Instead of the CRL bilens, we here demonstrate the approach of X-ray waveguide beamsplitters. In contrast to refractive or diffractive optics, X-ray waveguides are essentially non-dispersive optical components, and are thus also suitable for broader bandpass radiation and for ultra-short pulses. Furthermore, the beamsplitter can be combined with coherence (mode filtering) and the direction of the exit beams can be manipulated. Already in Fuhse et al. (2006) we have shown that two adjacent waveguide beams can be used for offaxis holographic imaging, but in that work the two channels did not emerge from a single input channel as we have now realized in the present work. An advanced fabrication scheme with improved lithography, etching and wafer bonding steps has now paved the way to realizing $X$-ray optics on a chip. Towards this goal, efficient X-ray beamsplitters as demonstrated here will be indispensable components.

\section{Experiments}

\subsection{Fabrication of beamsplitters}

The waveguiding structures were fabricated according to the protocol described by Neubauer et al. (2014) with further optimizations resulting in higher transmission values of the waveguide channels. A silicon wafer (MaTeck) with a diameter of $10 \mathrm{~cm}$ was covered with an electron-beam resist (ARP 679.04, Allresist) by spin coating (Optispin SB20, Sister Semiconductor Equipment $\mathrm{GmbH}$ ) the solution at $2000 \mathrm{r} \mathrm{min}^{-1}$ for $70 \mathrm{~s}$ followed by a post-baking step (453 K for $90 \mathrm{~s})$. The beamsplitting patterns were brought into the resist by electron-beam lithography (e-LiNE, Raith). A development procedure was performed, dipping the wafer into a mixture of methyl isobutyl ketone (MIBK) and 2-propanol (IPA) in a ratio of $1: 3$ at $273 \mathrm{~K}$ for $30 \mathrm{~s}$. To stop the removal of the resist, the wafer was immersed in pure IPA at room temperature and kept there for $30 \mathrm{~s}$. The electron-beam resist serves as a mask for reactive ion etching (PlasmaLab, Oxford) for $160 \mathrm{~s}$. The structures were transferred into the wafer utilizing $10 \mathrm{sccm}: 40 \mathrm{sccm}$ (where $\mathrm{sccm}=$ standard cubic centimetres per minute) of $\mathrm{SF}_{6}$ and $\mathrm{CHF}_{3}$ for etching and sidewall passivation (Legtenberg, 1995). After cleaning the etched wafer employing ammonia (32\%), hydrogen peroxide $(30 \%)$ and deionized water [1:1:5, RCA cleaning procedure (Kern \& Werner, 2008)], a second wafer was manually put on top of the first one, embedding the etched structures (see Fig. $1 b)$. The wafers were inspected using an infrared camera to detect voids in the contact surface of the wafers. The final wafer bonding took place in an oven (L 3/11/P330, Naber-
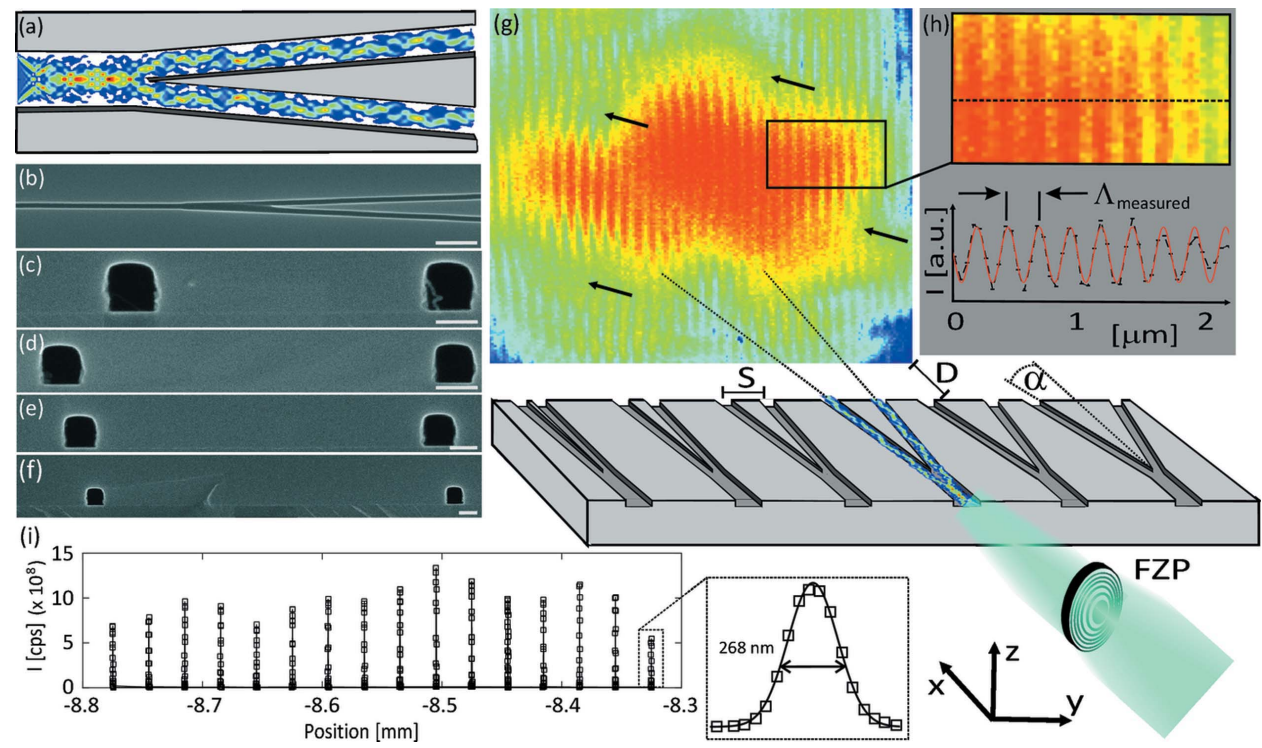

Figure 1

Experimental geometry. (a) Finite differences simulation of a beamsplitter. $(b)$ Top view SEM image of a splitting structure before wafer bonding. Scale bar denotes $1 \mu \mathrm{m}$. $(c)-(f)$ SEM images of the exit side of beamsplitters with different spacings $S$. Scale bars denote $100 \mathrm{~nm}$. ( $g$ ) Schematic of the experimental geometry showing the coupling of the focused X-ray beam into the entrance of the beamsplitter, the subsequent guiding in the two channels, the free-space propagation behind the chip, and finally the far-field detector at a distance $D$. The far-field pattern shows the characteristic double-slit interference pattern, modulated with features of the waveguide modal structure. Arrows mark bifurcations in the interference fringes (forkshaped structures). Length and angles are not to scale. ( $h$ ) Enlarged view of the interference pattern with a sinusoidal fit to the intensity oscillations. ( $i)$ Scan in the $y$ direction indicating the position of different beamsplitters which have all been defined on the same chip with different geometric parameters, and which can be selected by translating the chip in the FZP focus. Detailed scan profile of a single channel with a width (FWHM) of $282.6 \mathrm{~nm}$ giving an upper limit for the beam size in the horizontal direction. 
therm) in a nitrogen atmosphere at $1273 \mathrm{~K}$ for $4 \mathrm{~h}$, resulting in covalent bonds between the wafers. Finally the waveguide chip was diced (DAD320, Disco) to the chosen geometry, leaving the entrances and exits of the channels open (see Figs. 1c-1f).

\subsection{Experimental setup}

The experiments were carried out at the upgraded undulator beamline ID01 of the European Synchrotron Radiation Facility (ESRF). The monochromatic beam of $8 \mathrm{keV}$ photon energy [horizontal $\mathrm{Si}(111)$ double-crystal monochromator] was prefocused by a beryllium CRL with a radius of $500 \mu \mathrm{m}$ and then focused by a tungsten Fresnel zone plate (FZP) with outermost zone width $\Delta r=70 \mathrm{~nm}$ and diameter $300 \mu \mathrm{m}$. A central stop of $50 \mu \mathrm{m}$ and an order sorting aperture (OSA) were used to prepare a clean first-order focus with a spot size of $224 \mathrm{~nm}$ (horizontal) $\times 244 \mathrm{~nm}$ (vertical) (full width at halfmaximum, FWHM), measured by scanning a straight channel with a size of $170 \times 114 \mathrm{~nm}$ across the beam. The primary intensity of the beam was $I_{0} \simeq 1.11^{10}$ counts s$^{-1}$ for a ring current of $188 \mathrm{~mA}$. The waveguide chip was aligned in the focal spot of the FZP, using three lateral and three rotational degrees of freedom, enabling an optimized perpendicular coupling of the beam into the front side of the waveguide channel. At $D=1.386 \mathrm{~m}$ behind the chip, the far-field patterns were recorded by a two-dimensional pixel detector (Maxipix, ESRF, with $516 \times 516$ pixels with a size of $55 \times 55 \mu \mathrm{m})(\mathrm{see}$ Fig. 1i).

Fig. 1(b) illustrates the splitting geometry showing a top view of a channel with $S=30 \mu \mathrm{m}$. For the channels investigated here, the opening angle $\alpha$ of the two channels is in the order of 1-2.5 mrad. Figs. 1(c)-1(f) show scanning electron microscopy (SEM) images of the exit side of several beamsplitters with different channel spacings $S$. Examples with spacings from 0.774 to $1.750 \mu \mathrm{m}$ are presented. In Fig. $1(\mathrm{~g})$ a schematic of the measurement setup is displayed. The beam is focused by an FZP and coupled into the beamsplitter at the focal spot. When propagating in the splitter, the beam is split into two channels, each with a width of $103 \times 118 \mathrm{~nm}$, corresponding to half of the area of the in-coupling region. After exiting the waveguide chip, the two X-ray beams propagate in air and interfere along the way towards the
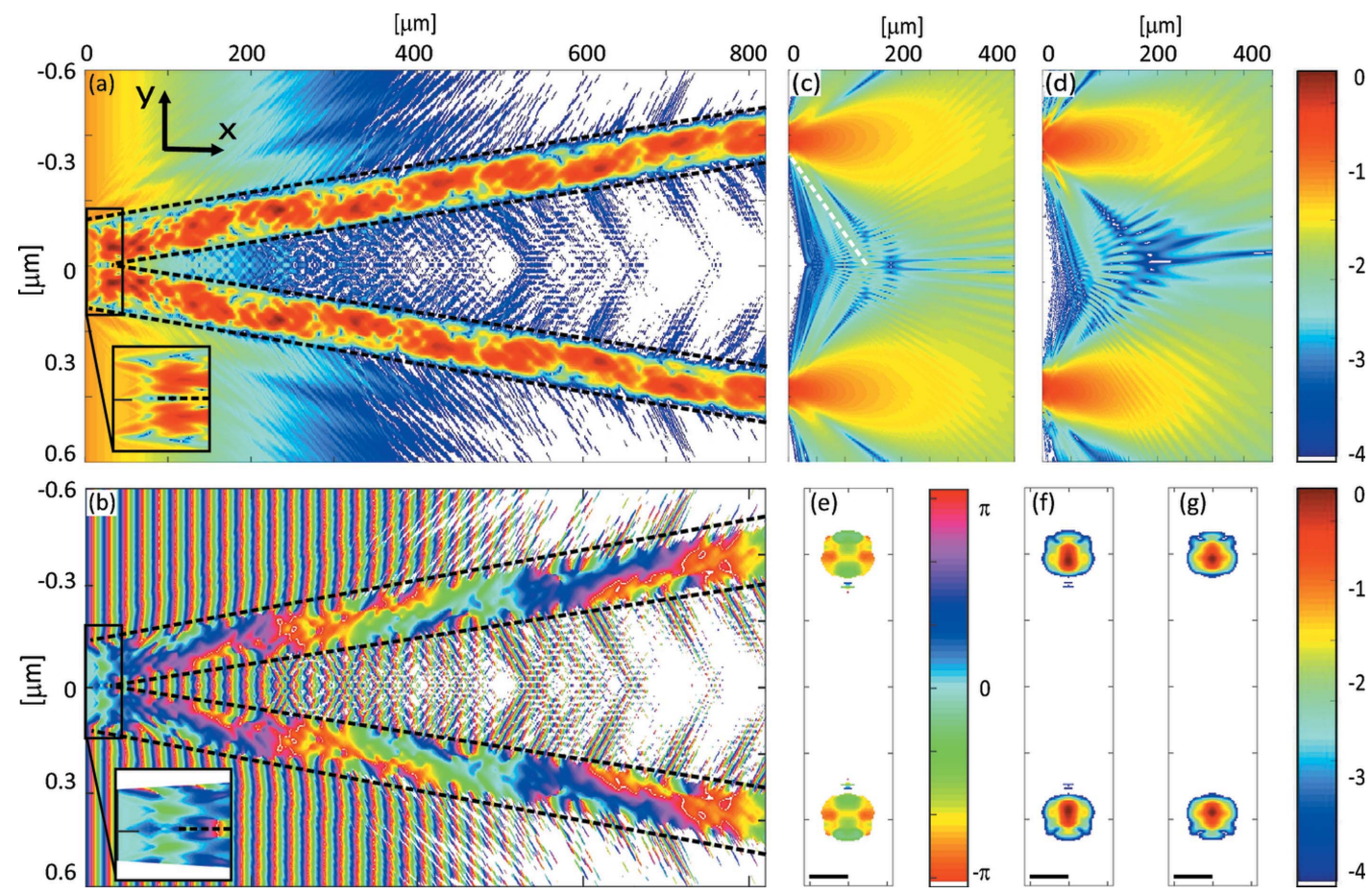

Figure 2

Finite differences simulations. (a) Intensity of a wavefield within the waveguide. The dashed lines mark the channel boundaries. The enlarged part displays the region where the wave is coupled in; note the large aspect ratio of 1:100. (b) Phase of the guided radiation inside the structure. Near-field propagations of the exit fields are shown for the simulated beamsplitter $(c)$ and for a wavefield reconstructed from experimental far-field data $(d)$. Cross sections of the wavefield $(a)$ in the channels, shown in phase $(e)$ and in intensity $(f)$ at $1 \mu \mathrm{m}$ upstream from the waveguide exit. $(g)$ Slice through the propagated near-field, at a distance of $2 \mu \mathrm{m}$ behind the waveguide exit. For $(e)-(g)$ the scale bars denote $0.1 \mu \mathrm{m}$. All intensities are normalized and plotted on a logarithmic scale; the phases are given in units of radian. 


\section{research papers}

Table 1

Beamsplitters with various spacings $S$ of the exit channels and the associated transmission values.

The design period $\Lambda_{\text {designed }}$ at the detector is calculated from geometric relations. Note that periods $\Lambda_{\text {measured }}<110 \mu \mathrm{m}$ cannot be resolved, since the pixel size of the detector is $55 \mu \mathrm{m}$.

\begin{tabular}{llll}
\hline Spacing $S(\mu \mathrm{m})$ & $\Lambda_{\text {designed }}(\mu \mathrm{m})$ & $\Lambda_{\text {measured }}(\mu \mathrm{m})$ & Transmission $(\%)$ \\
\hline 0.774 & 277.582 & 268.303 & 26.68 \\
0.961 & 223.568 & 216.160 & 32.90 \\
1.100 & 195.317 & 191.161 & 28.77 \\
1.400 & 153.463 & 155.889 & 24.39 \\
1.550 & 138.612 & 139.603 & 35.13 \\
1.750 & 122.771 & 126.411 & 26.48 \\
1.920 & 111.900 & & 29.75 \\
2.110 & 101.824 & & 21.62 \\
2.300 & 93.413 & & 21.65 \\
2.500 & 85.940 & & 22.28 \\
2.700 & 79.574 & & 19.67 \\
2.900 & 74.086 & & 16.09 \\
3.100 & 69.306 & & 21.31 \\
3.300 & 65.106 & & 20.35 \\
3.500 & 61.385 & & 19.04 \\
3.900 & 55.089 & & 16.79
\end{tabular}

detector, developing the far-field pattern of alternating vertical stripes as expected from a classical double-slit interference (Young, 1802). The distance between two neighbouring maxima $\Lambda_{\text {measured }}$ is quantified by a sinusoidal fit to the intensity profiles (Fig. $1 h$ ). On closer examination, fork-like structures are observed in some locations in the pattern (marked with arrows), appearing as defects of the otherwise regular sinusoidal oscillations. These features are associated with discontinuities in the phase as investigated further below. The low-frequency oscillations in the pattern arise from the mode structure of the beams which will be discussed in the next section. Several beamsplitters on one chip are scanned along in the lateral direction (Fig. 1i). Table 1 lists the complete data set of all measured beamsplitters. The size of the incoupled beam can be rechecked from a Gaussian fit to a scan using one splitter, yielding an FWHM of $282.6 \mathrm{~nm}$. Since the entrance width of the beamsplitter is larger, this value is slightly larger than $224 \mathrm{~nm}$ - estimated from a scan with a straight reference channel (see the description of the setup).

For beamsplitters with a spacing $S>1.75 \mu \mathrm{m}$, the interference pattern cannot be resolved at the detector, since the oscillation period $\Lambda_{\text {measured }}$ in the pattern is below $110 \mu \mathrm{m}$ and the pixel size is $55 \mu \mathrm{m}$. From geometric relations along with the condition for constructive interference, the period of the interference pattern at the distance of the detector can be calculated using the design spacings $S$ verified by SEM inspection. The design period of the diffraction pattern is $\Lambda_{\text {designed }}=\lambda \times D / S$, where $\lambda=0.155 \mathrm{~nm}$ denotes the wavelength and $D$ the distance between waveguide and detector. To obtain the experimental value $\Lambda_{\text {measured }}, 20$ neighbouring slices in the measured far-field pattern are horizontally summed, followed by a subtraction of the multiple Gaussianshaped background. The latter is the result of the lowfrequency mode structure (see Fig. 1g). Afterwards, the oscillations are fitted to a sine function. The deviations between the design and measured periods are smaller than
$4.2 \%$. The transmission of the splitters is calculated by comparing the intensity of the (FZP) beam to the detected farfield pattern of the waveguide, both normalized by the beam size and channel size of the entrance side, respectively. Depending on the channel, the transmission lies in the range of $16-35 \%$ which is one third of the theoretical value of $93.9 \%$ at $8 \mathrm{keV}$. This difference can be explained by the fact that the channel walls are assumed to be atomically flat in the simulation, but show micro roughnesses in the range of a few nanometres (Osterhoff \& Salditt, 2009). Furthermore, roughness and imperfections in the splitting region may lead to additional loss mechanisms not accounted for in the idealistic simulations. Exemplarily, the analysis for a channel with $S=0.774 \mu \mathrm{m}$ will now be presented in more detail, starting with numerical simulations of the wavefield inside and outside the channels.

\section{Numerical simulations of beamsplitters}

Fig. 2 presents the finite differences simulations (Melchior, 2016) of the beamsplitter with $S=0.774 \mathrm{~nm}$. In Figs. 2(a) and 2(b) intensity and phase inside the waveguide are plotted. Waves impinging on the waveguide chip are successively absorbed by the silicon cladding. Within the guiding core that is filled with air, the waves propagate through the $820 \mu \mathrm{m}$-long chip. Impinging the channel boundaries beneath the critical angle $\alpha_{\text {crit. }}=0.227^{\circ}$ (Henke et al., 1993), the radiation is totally reflected and thus guided through the splitter. When exceeding the critical angle, the radiation leaks into the cladding and is absorbed as well. This is clearly visible after a guiding distance of $400 \mu \mathrm{m}$ (see Figs. $2 a, 2 b$ ). The angle of the $\mathrm{X}$-rays leaking into the cladding can be graphically estimated to a value of $0.4^{\circ}$, which is clearly above the critical angle. The $\mathrm{X}$-rays leaving the channels at the exit side show a coneshaped geometry by diffraction while propagating in free space; see the free-space simulations by Fresnel propagation of the exit field shown in Fig. 2(c). As input for the Fresnel propagation, we chose a combination of the exit wavefields shown in Figs. $2(f)$ and $2(g)$, corresponding to a slightly different chip length of $3 \mu \mathrm{m}$, which can occur if the splitter geometry or the dicing plane are not perfect. In fact, this choice (of slightly different channel length) gave the best agreement with the propagation calculated for the experimental near-field reconstructions shown in Fig. 2(d) for comparison. The beam propagation along the optical axis as shown in Fig. 2(c) indicates that the two cones start to interfere after a propagation distance of about $200 \mu \mathrm{m}$, with the exceptions of side streaks departing at higher angles (marked by a dashed line), which already interfere after about $120 \mu \mathrm{m}$. In contrast, for the near-field using the reconstructed exit wave from the measured far-field data (Fig. $2 d$ ), the cones interfere only after a distance of about $350 \mu \mathrm{m}$. Similarly to the nearfield in Fig. 2(c), a streak is visible that intersects the optical axis at about $90 \mu \mathrm{m}$. As we discuss below, the opening angle of diffraction depends on the confinement of the beam in the exit plane, as given by the multi-modal interference rather than the pure geometric cross section of the channel. This is illustrated 
in Figs. 2(e) and 2(f), showing intersections of the simulated intensity and corresponding phase in a plane $1 \mu \mathrm{m}$ upstream from the exit plane of the waveguide. The exit waves of both channels exhibit a flat phase and size of about $42 \times 26 \mathrm{~nm}$, indicating an elongation in the $y$ direction. These values represent the FWHM quantified from Gaussian fits to the intensity distributions in the $y$ and $z$ directions. The shape of the beam still changes while propagating over the last microns to the exit plane, owing to the complex mode structure inside the channel. At $2 \mu \mathrm{m}$ behind the waveguide exit (Fig. $2 g$ ), the beam has a size of $35 \times 28 \mathrm{~nm}$, yielding an almost circular cross section. Unconfined in free space, the shape of the beam broadens as expected for propagation.

Next, we briefly cross-check by simple analytical calculation the consistency of the values for beam divergence $\Theta$ (half angle of the radiation cone) and beam confinement $d_{\text {beam }}$ (FWHM), in particular for the wavefield reconstructed from the experimental far-field pattern. To this end, we compare our values with the analytical solution of a Gaussian beam. Even if a multi-modal waveguide beam is not particularly well approximated by this simple model, it provides a useful benchmark. Accordingly, the intensity in the exit plane can be described as $I(x, r)=I_{0}(x) \exp \left[-2 r^{2} / w(x)^{2}\right]$, where $I_{0}$ is the peak field intensity, $r=\left(y^{2}+z^{2}\right)^{1 / 2}$ is the radial distance from the centre, $w_{0}$ is the beam waist radius and $w(x)$ is the halfwidth parameter, where the intensity $I(x, 0)$ has decayed by a factor of $1 / e^{2}$ (Saleh $\&$ Teich, 1991). At a distance $x_{0}$ the beam intensity has decayed to half of its maximum; the beam radius is $r=d_{\text {beam }} / 2$ and $w\left(x_{0}\right)=2^{1 / 2} w_{0}$. The half opening angle $\Theta=\lambda / \pi w_{0}$ of the intensity cones can be calculated from the wavelength $\lambda$ and the beam size in the exit plane of the waveguide $d_{\text {beam }}$ :

$$
\Theta=\frac{\lambda}{\pi w_{0}}=\frac{\lambda 2(\ln 2)^{1 / 2}}{\pi d_{\text {beam }}} .
$$

In the simulated case, a beam size $d_{\text {beam }}$ of about $45 \mathrm{~nm}$ in the $y$ direction results in an opening angle of $\Theta_{\text {sim }}=1.8 \mathrm{mrad}$. In the two-beam setup this means that for $S=0.774 \mathrm{~nm}$ the beams begin to intersect (and hence to interfere) at a distance of $190 \mu \mathrm{m}$, in good agreement with the observed $200 \mu \mathrm{m}$ (see Fig. 2c). For the field reconstructed from the measurements, however, the beam sizes of the two channels are actually different, namely 20 and $70 \mathrm{~nm}$, as shown in the following section, where the field reconstruction is presented.

\section{Near-field reconstruction}

In this section, the evolution of the near-field will be analysed by retrieving the wavefield at the exit side of the channels from the measured far-field pattern, using an error reduction algorithm (Fienup, 1982). Fig. 3(a) presents the reconstructed wavefield at the exit side of the waveguide that is overlaid with the corresponding SEM image. For the reconstruction, a support of two rectangular shaped boxes with the size and distance according to the SEM measurements was chosen and 500 iterations were performed. The two reconstructed X-ray beams exhibit a size of about $22 \times 52 \mathrm{~nm}$ and $77 \times 67 \mathrm{~nm}$ (FWHM), respectively. Hence, while the left one shows an elongation in the $z$ direction, the right one is elongated along $y$
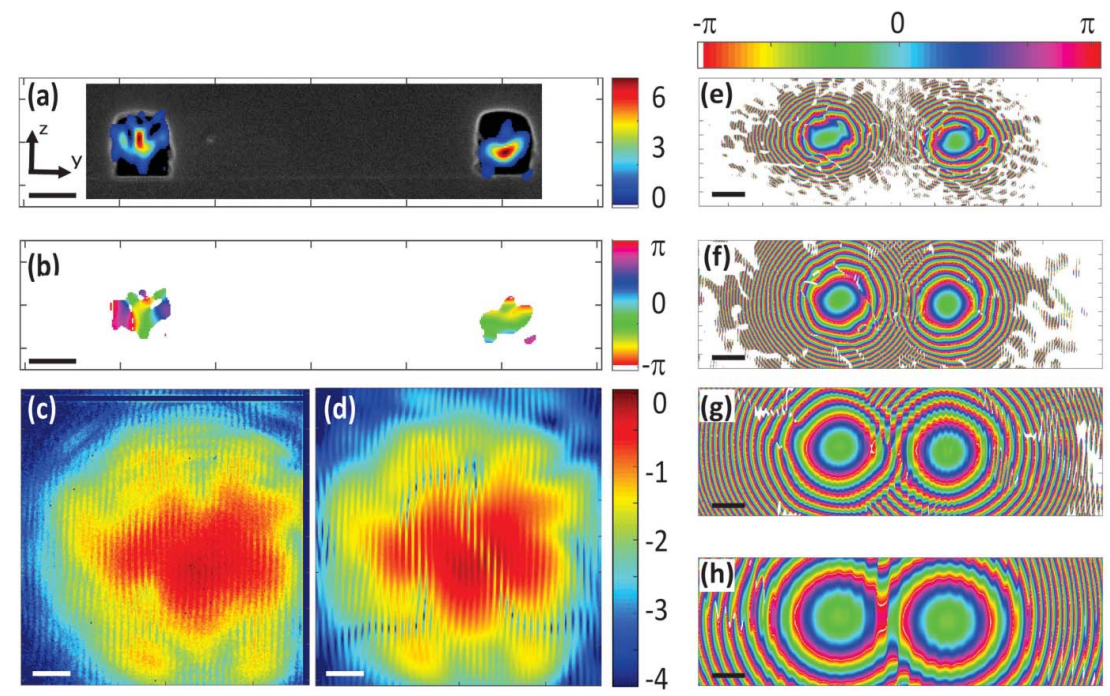

$-2$

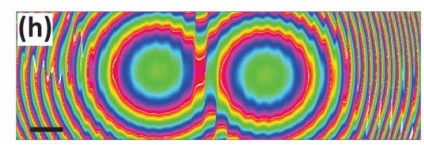

Figure 3

Reconstructions. (a) The reconstructed wavefield overlaid with the SEM image at the exit plane of the sample and the corresponding phase (b); scale bars denote $0.1 \mu \mathrm{m}$. (c) Measured far-field and $(d)$ reconstructed far-field both in logarithmic scaling; scale bars denote $2 \mathrm{~mm}$. $(e)-(h)$ Slices through the near-field at 50,100, 200 and $400 \mu \mathrm{m}$ behind the sample, respectively. Scale bars denote $0.2 \mu \mathrm{m}$; the phases are given in units of radian. 


\section{research papers}

(Fig. 3a). As described in the previous section, from a difference in the propagation length of only a few microns, a rotation of the orientation in this manner may arise. The size and the shape of the field correspond to the simulations shown in Figs. $2(f)$ and $2(g)$. Furthermore, Fig. 3(b) shows that the phase distributions in the source plane are flat in the regions where the intensity is confined. In Figs. 3(c) and 3(d) the measured and reconstructed far-field patterns are displayed. The general shape of the intensity distribution and the frequency of the oscillations are reproduced very well (see also Table 1). This is quantified by a deviation of $1.4 \%$ between the two far-field patterns (relative deviation averaged over all pixels). As a further confirmation of the successful reconstruction, we see that the algorithm has even reconstructed reasonable intensities in the regions of the detector module gaps, where no data were measured. The phase of the propagated radiation is displayed at different distances in the near-field to demonstrate how the interference of the two beams evolves. At $50 \mu \mathrm{m}$ the two intensity cones have not yet overlapped (Fig. 3e). After a propagation length of $100 \mu \mathrm{m}$ the beams begin to interfere. In this process the concentric phase distributions intersect each other leading to discontinuities in the phase field. At $200 \mu \mathrm{m}$ (Fig. $3 g$ ) the cones start to interact and at $400 \mu \mathrm{m}$ (Fig. $3 h$ ) the far-fields have penetrated each other completely. The far-field is fully developed and does not change in general shape anymore, but only grows larger during further propagation.

\section{Phase vortices}

For distances between 200 and $400 \mu \mathrm{m}$ behind the waveguide exit, shown in Figs. $4(a)-4(d)$, the intensity and the contour lines of the phases (iso-phases, at $2 \pi$ intervals) are plotted simultaneously. The position of one phase discontinuity is followed in three dimensions through the propagation, indicated by an arrow. At first, the discontinuity moves upwards between two intensity fringes. In Fig. 4(c) a forklike structure begins to form and is fully developed in Fig. 4(d). On closer examination, the contour lines are discontinuous forming a dead end, see Figs. $4(d)$ and $4(f)$. The contour lines end in intensity minima (core). Comparing the intensity distribution and phase (see Figs. $4 e$ and $4 f$ ), discontinuities in the phase are accompanied by zeros in the intensity (White et al., 1991; Baumann \& Galvez, 2007). To display this more clearly, an intensity profile perpendicular to the grating direction along the line is shown in Fig. $4(\mathrm{~g})$; the corresponding phase is plotted on the right axis. In general, the phase discontinuities deform the intensity pattern by bending the fringes (Nye, 1999; Bogatyryova et al., 2003). If steep phase gradients occur at positions where there is a maximum in the inter-

Vortex analysis. $(a)-(d)$ Near-field at 200, 250, 300 and $400 \mu \mathrm{m}$ behind the waveguide exit, respectively. The arrows mark the evolution of a phase discontinuity; scale bars denote $0.1 \mu \mathrm{m}$. $(e)$ Intensity and $(f)$ phase at a distance of $400 \mu \mathrm{m}$ behind the waveguide exit. The black arrow marks the same fork as in $(d)$ and the grey arrow indicates the position of the anti-vortex; both are additionally highlighted in the insets. $(g)$ Intensity profile extracted from $(e)$ along the line. The corresponding phase is shown on the right axis. $(h)$ Phase profiles from $(f)$ along the dashed paths in the enlarged views. 
ference pattern, the wavefield forms an extra fringe. In those forks one fringe is twinned, which can be described analogously to an edge dislocation (Nye \& Berry, 1974) with a phase singularity at the centre (core). This optical phenomenon is called a phase vortex. At the core, the complex amplitude equals zero and the phase is not defined (Kitchen et al., 2004). In Fig. $4(f)$ the region of the core and a closed loop $L$ around it are shown in higher magnification. Furthermore, we can observe an anti-vortex associated with the vortex described above (see grey arrow). At this vortex, the phase winds in the opposite direction - relative to the first vortex, see also the insets. Two neighbouring vortices with opposite helicity are indicative for a characteristic 'hairpin' dislocation line, piercing twice through the same optical plane (Berry, 1998; Dennis et al., 2009). In Fig. $4(h)$ the phase $\Phi$ plotted along $L$ shows the expected phase jumps of $2 \pi$ when circuiting the vortices. According to Takahashi et al. (2013) we can write

$$
\oint \nabla \Phi \times d L=2 \pi m \quad \text { with } \quad m= \pm 1, \pm 2, \pm 3, \ldots
$$

where $m$ is called the topological charge of the singularity (Gbur \& Visser, 2003). For the vortex depicted in Fig. 4(f) $m=$ +1 (Basistiy et al., 1995) and this is (apart from the sign) also the case for all vortices in the entire far-field, since only forks with two rakes were observed. The anti-vortex carries the opposite charge. Optical vortices are quite common in the regime of visible wavelengths (Coullet et al., 1989; White et al., 1991) and were described for the first time for X-rays by Peele et al. (2002).

\section{Conclusion}

We have studied the optical performance of waveguide beamsplitters by recording far-field patterns and carrying out subsequent reconstructions of the near-field by phase retrieval techniques. In this way, we could analyse characteristic structures of the optical field, in particular the evolution of interference fringes and phase vortices. Complementing the previously demonstrated two-dimensionally confined channels in silicon in straight (Neubauer et al., 2014; Bartels et al., 2015) and tapered geometries (Chen et al., 2015), we have hence demonstrated beamsplitters as advanced X-ray optics with a precisely controlled exit spacing. The efficient transport of radiation by guiding in the beamsplitters, as first studied by numerical simulation, was perfectly confirmed experimentally. Notably, the near-field reconstructed from the recorded farfield pattern was found to be in good agreement with the simulations, assuming a slight asymmetry in the length of about $3 \mu \mathrm{m}$ between the two exit channels. The details reproduced from the phase retrieval and its robustness were surprising, given the fact that single-waveguide field reconstructions by the error reduction algorithms often exhibit high sensitivity to initialization and the precise choice of the support. In contrast, we experienced a much higher reliability of the phase retrieval in the present scenario of two interfering beams. This can be taken as an indication that the structured illumination of samples by the two beams may possibly provide some interesting advantages for coherent imaging. Certainly, the beamsplitters provide a novel tool for interferometry and absolute determination of the phase (Momose, 1995). In contrast to the interferometer based on two adjacent nano-CRLs (Snigirev et al., 2009), the present system can be designed such that the mode structure is filtered and hence coherence properties can be decoupled from the synchrotron source. In the present experiment, we also found intricate phase vortices, which are formed by the intersection of the two beams. The observation of these interesting phase features is clearly facilitated by the above-mentioned robust phase retrieval setting, offering new perspectives for future work. More geared towards applications, the beamsplitters could be multiplied to deliver several daughter beams, all from the same mother, in such a way that a single object could be imaged by several beams, each with a different direction. The required curvature of the segments can be easily supported by waveguiding if the channels are sufficiently narrow, as we have shown recently (Salditt et al., 2015). Also, controlled structures for time delay of ultra-fast free-electron laser pulses are conceivable based on splitters and curved elements.

\section{Acknowledgements}

We thank Dr Anna-Lena Robisch for advice and help with the reconstruction and propagation methods, Lars Melchior for his enabling software for finite difference calculations, Malte Vassholz for help during beamtime, Jan Hilhorst for excellent user support at ID01, the ESRF for beamtime, the Helmholtz Society for funding through grant No. VH-VI-403 and the BMBF for grant No. 05K16MGB.

\section{References}

Bartels, M., Krenkel, M., Haber, J., Wilke, R. \& Salditt, T. (2015). Phys. Rev. Lett. 114, 048103.

Basistiy, I., Soskin, M. \& Vasnetsov, M. (1995). Opt. Commun. 119 604-612.

Baumann, S. \& Galvez, E. (2007). Proc. SPIE, 6483, Complex Light and Optical Forces, 64830T. doi:10.1117/12.701638.

Berry, M. (1998). Proc. SPIE, 3487, International Conference on Singular Optics, 6. doi:10.1117/12.317704.

Bogatyryova, G., Fel'de, C. V., Polyanskii, P., Ponomarenko, S. Soskin, M. \& Wolf, E. (2003). Opt. Lett. 28, 878-880.

Bongaerts, J., Zwanenburg, M., Zontone, F. \& van der Veen, J. (2001). J. Appl. Phys. 90, 94-100.

Bonse, U. \& Hart, M. (1965). Appl. Phys. Lett. 6, 155-156.

Chen, H., Hoffmann, S. \& Salditt, T. (2015). Appl. Phys. Lett. 106, 194105.

Coullet, P., Gil, L. \& Rocca, F. (1989). Opt. Commun. 73, 403-408.

Dennis, M. R., O'Holleran, K. \& Padgett, M. J. (2009). Prog. Opt. 53 293-363.

Feng, Y., Sinha, S., Fullerton, E. E., Grübel, G., Abernathy, D., Siddons, D. \& Hastings, J. (1995). Appl. Phys. Lett. 67, 3647-3649. Fienup, J. R. (1982). Appl. Opt. 21, 2758-2769.

Fuhse, C., Ollinger, C. \& Salditt, T. (2006). Phys. Rev. Lett. 97, 254801 Gbur, G. \& Visser, T. (2003). Opt. Commun. 222, 117-125.

Henke, B., Gullikson, E. \& Davis, J. (1993). At. Data Nucl. Data Tables, 54, 181-342.

Jarre, A., Fuhse, C., Ollinger, C., Seeger, J., Tucoulou, R. \& Salditt, T. (2005). Phys. Rev. Lett. 94, 074801. 
Kern, K. \& Werner, A. (2008). Editors. Handbook of Silicon Wafer Cleaning Technology, 2nd ed. Norwich, New York, USA: William Andrew Publishing.

Kitchen, M., Paganin, D., Lewis, R., Yagi, N., Uesugi, K. \& Mudie, S. (2004). Phys. Med. Biol. 49, 4335-4348.

Lagomarsino, S., Jark, W., Di Fonzo, S., Cedola, A., Mueller, B., Engström, P. \& Riekel, C. (1996). J. Appl. Phys. 79, 4471-4473.

Legtenberg, R. (1995). J. Electrochem. Soc. 142, 2020-2028.

Melchior, L. (2016). Pypropagate. https:/github.com/TheLartians/ PyPropagate

Momose, A. (1995). Nucl. Instrum. Methods Phys. Res. Sect. A, 352 $622-628$.

Neubauer, H., Hoffmann, S., Kanbach, M., Haber, J., Kalbfleisch, S., Krüger, S. \& Salditt, T. (2014). J. Appl. Phys. 115, 214305.

Nye, J. F. (1999). Natural Focusing and Fine Structure of Light: Caustics and Wave Dislocations. London: CRC Press.

Nye, J. \& Berry, M. (1974). Proc. R. Soc. A Math. Phys. Eng. Sci. 336, $165-190$.

Osterhoff, M. \& Salditt, T. (2009). Opt. Commun. 282, 3250-3256.

Osterhoff, M. \& Salditt, T. (2011). New J. Phys. 13, 103026.

Peele, A. G., McMahon, P., Paterson, D., Tran, C., Mancuso, A., Nugent, K., Hayes, J., Harvey, E., Lai, B. \& McNulty, I. (2002). Opt. Lett. 27, 1752-1754.
Pelliccia, D., Cedola, A., Scarinci, F. \& Lagomarsino, S. (2005). J. Phys. D Appl. Phys. 38, A213.

Pfeiffer, F., David, C., Burghammer, M., Riekel, C. \& Salditt, T. (2002). Science, 297, 230-234.

Pfeiffer, F., Salditt, T., Høghøj, P., Anderson, I. \& Schell, N. (2000). Phys. Rev. B, 62, 16939-16943.

Prudnikov, I. (2003). Phys. Rev. B, 67, 233303.

Salditt, T., Hoffmann, S., Vassholz, M., Haber, J., Osterhoff, M. \& Hilhorst, J. (2015). Phys. Rev. Lett. 115, 203902.

Saleh, B. E. A. \& Teich, M. C. (1991). Fundamentals of Photonics. New York: Wiley.

Snigirev, A., Snigireva, I., Kohn, V., Yunkin, V., Kuznetsov, S., Grigoriev, M., Roth, T., Vaughan, G. \& Detlefs, C. (2009). Phys. Rev. Lett. 103, 064801.

Spiller, E. (1974). Appl. Phys. Lett. 24, 60-61.

Takahashi, Y., Suzuki, A., Furutaku, S., Yamauchi, K., Kohmura, Y. \& Ishikawa, T. (2013). Phys. Rev. B, 87, 121201.

White, A., Smith, C., Heckenberg, N., Rubinsztein-Dunlop, H. McDuff, R., Weiss, C. \& Tamm, C. (1991). J. Mod. Opt. 38, 25312541.

Young, T. (1802). Philos. Trans. R. Soc. London, 92, 387-397.

Zwanenburg, M., Peters, J., Bongaerts, J., de Vries, S., Abernathy, D. \& van der Veen, J. (1999). Phys. Rev. Lett. 82, 1696-1699. 



\section{Chapter 7}

\section{Summary and concluding remarks}

In this thesis, the fabrication and testing of two-dimensionally confined x-ray waveguides is presented involving e-beam lithography, reactive ion etching and wafer bonding techniques (see chapter 2). The waveguides characterized here are front-coupling devices consisting of an air-filled channel imbedded in a homogeneous silicon cladding. Optimizations of the parameters of all process steps led to a fabrication scheme capable of producing waveguides in advanced geometries as well as arbitrary dimensions down to cross-sectional areas in the range of $40 \times 40 \mathrm{~nm}^{2}$ [see Chap., Fig. 1(e)]. As shown in section 1.1. channels of these dimensions filter out one or several modes from the incoming wave field, while the cladding absorbs evanescent radiation not guided within the channel. To avoid parasitic radiation 7 large aspect ratios of the waveguide channel lengths in the range up to $1: 10^{6}$ are required. Although the channel length is designed to absorb the radiation impinging onto the cladding for a certain wavelength, they are also suitable for lower energies.

The wave fields in the exit plane of the waveguide channels, exhibit well defined dimensions of regions of highest intensity (modes) as well as flat phases, both evidenced by reconstructions from the detected far-field patterns using the error reduction algorithm (section 1.3. A coherent probe without wavefront errors is most suitable for high resolution x-ray imaging. The near-field intensity distributions for various channel sizes and energies are in agreement with the finite difference simulations and the electron-microscopy data, showing the high reliability of the gained results. The retrieval of the optical near-field is followed by propagation studies.

Besides single straight channels, a multitude of different geometries such as arrays of parallel channels, beamsplitters, curved and tapered channels were fabricated and their functionality was experimentally proven. Regarding the progress in optical performance, straight waveguides with $100 \times 100 \mathrm{~nm}^{2}$ channel size were fabricated with

7 For hard x-rays the transmission of an air filled channel $(L \leq 2 \mathrm{~cm})$ is $T_{\text {air }} \approx 1$, while the absorbing cladding ( $T_{S i} \approx 10^{-7}$ ) ensures for a sufficient signal-to-noise ratio. 
transmissions of $T=30 \%$, note that the actual source size is smaller than $100 \mathrm{~nm}$ by mode confinement [see regions of high intensity in Fig 1.17 (c)]. Compared to the efficiencies in table 1.1 waveguides are among the best performing optics demonstrating the accuracy of the fabrication. Far-field patterns of channels with a large number of different cross-sections are depicted within this work which clearly illustrates the filtering process. While maintaining this property, a concentration of the wave field is additionally enabled by applying a tapered geometry as shown analytically and confirmed by experiments $82,50,83$. The field enhancement is achieved by funneling a large entrance in the micron range down to an nanometer-sized exit [84]. Building on the highquality straight structures, tapered waveguides with a similar exit size of $98 \times 83 \mathrm{~nm}^{2}$ revealed a virtual source as small as $23 \times 24 \mathrm{~nm}^{2}$ (FWHM), while the intensity at the detector was $I=1.45 \cdot 10^{10} \mathrm{cps}$, corresponding to a transmission of $T=40 \%$ (see Fig 1.10 . Amongst other things, the variety of possible geometries is demonstrated in form of curved channels, which offered an x-ray beam deflected by $0.6^{\circ}$ from the optical axis (see Fig. 1.12. Unlike in previous publications [71], channels confined in two dimensions are presented in chapters 1.2.4 and 3. Hence, the waveguide exit field and the primary beam are clearly separated for the first time. Furthermore, gratings and beamsplitters are characterized, revealing double-slit far-field patterns with well controlled periodicities as well as phase singularities as interesting optical features. As argued in chapter 6, optical vortices are a well known and extensively examined subject in the regime of visible light, but are less common in x-ray optics. In the following paragraph, the view on vortices is completed in a more figuratively way and investigations on these phase singularities in the $\mathrm{x}$-ray regime are presented.
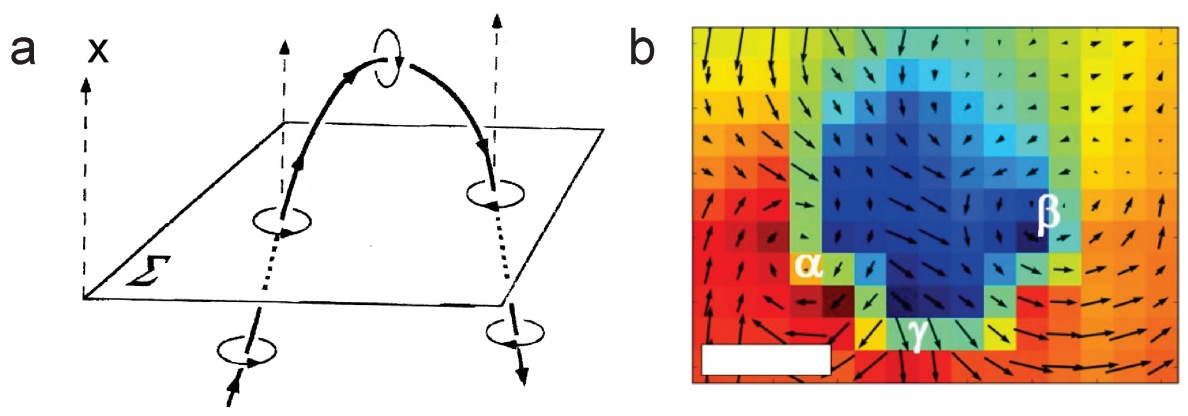

Figure 7.1: Phase vortices. (a) Schematic of a hairpin dislocation, reprinted from [85], with adaption to the nomenclature within this work. (b) Experimentally reconstructed multi-valued phase map (modulo $2 \pi$ ) in nominal focal plane $2.0 \mathrm{~mm}$ downstream of $x$-ray nanolenses, showing opposite-helicity branch points $\alpha$ and $\beta$ coincident with an $x$-ray vortex $(\beta)$ and antivortex $(\alpha)$. Overlaid is a vector field, showing the vortical behavior of the $x$-ray energy flow around the vortex-antivortex dipole, with a separation of $320 \mathrm{~nm}$. Scale bar denotes $150 \mathrm{~nm}$. Image and caption are reproduced from [86].

In figure 7.1(a), the structure of a hairpin dislocation is depicted. A curved dislocation line pierces the same optical plane $\sum$ twice, inducing two phase vortices with an opposite helicity. The sense in which the phase increases, is marked by a loop surrounding the singularity. This kind of phase singularity is characterized in chapter 6 , where the feature is not only analyzed in a fixed far-field plane but also its evolution is followed 
up in three dimensions while the wave field is propagated (see Fig. 3). In doing so, the intensity distribution in relation to the phase dislocations is investigated. In agreement with the theoretical framework, phase singularities are connected to zeros in intensity, since the phase is not defined when $\Psi=0$. However, in literature, other parts of the wave field were not considered in detail so far. In particular, it is observed here that (steep) gradients in the phase are correlated with minima in intensity (see Fig. 6.4). This is quantified by comparing profiles of the phase and intensity distribution obtained at the same position through the wave field. For each of the two vortices the predicted phase jump of $2 \pi$ or $-2 \pi$, respectively, was verified by studying a closed loop in the phase field around the singularity. Revealing opposite helicity of the phase, the two features are clearly identified as vortex and associated antivortex. The appearance of the wave field in this region is similar to the vortex/antivortex pair in the data set in figure 7.1(b). There, white arrows point in the direction of the energy flux in the field, adequately qualifying the sense of rotation of the phase.

Starting in 1950 [87, vortices were firstly recognized as an interesting optical phenomenon and their generation and characterization has been an often frequented topic since then. Making use of the unique properties of this feature, vortices are nowadays used for manipulation of matter, as for example in the form of optical tweezers and traps 88 .

Exploiting the small size of the channels, x-ray waveguides can also be used as diagnostic tools to verify that the optical instruments perform properly (see section 1.4). Due to their dimension they can for example be used for knife edge scans.

The nano-sized quartz channels can also be applied in other research areas, for example as nanofluidic devices using quartz only, or in combination with silicon [89] and also to investigate biomolecular dynamics such as unfolding of single DNA-molecules [90]. The adaption of the process steps to the insulating quartz was managed by using an additional (conducting) resist during lithography [91]. Germanium on the other hand can be utilized to fabricate micron-sized (hard) x-ray apertures with a high quality surface and tunable dimension, to efficiently block higher diffraction orders or the primary beam, thus providing an improved signal-to-noise ratio. Transferring the manufacturing process from silicon to germanium enabled also the fabrication of waveguides with a much shorter length, for a comparable channel quality (see chapter 3 . 92]), hence, less affected by real-structure effects such as surface roughness. The latter limits the transmission of waveguide channels and is introduced by inaccuracies within the fabrication process at the lithography, development and etching steps. To a minor degree, those defects are compensated by diffusion during the high temperature annealing, which however affects a shrinkage and even may cause blocked regions within the channel. The surface quality may be enhanced by anisotropic etching. For Si $\{110\}$ a potassium hydroxide $(\mathrm{KOH})$ dip results in steep and smooth vertical profiles 93 .

For roughness not smoothed out during the heating step or removed by anisotropic etching, finite difference simulations may help to evaluate the impact of the defective structures in more detail [66]. For this, a new model has to be developed to implement roughness on different length scales into the numerical simulations. 
In summary, the precisely controlled fabrication of waveguides enables the adaptation of the channel dimensions to the specific experimental requirements in highresolution x-ray imaging, and furthermore, it provides a first step towards optics on a chip [71] in the form of reference beam holography, interferometry, time delay or multiple-beam measurements, all making use of the flexibility in defining the channels path. 


\section{Appendix A}

\section{The waveguide universe}

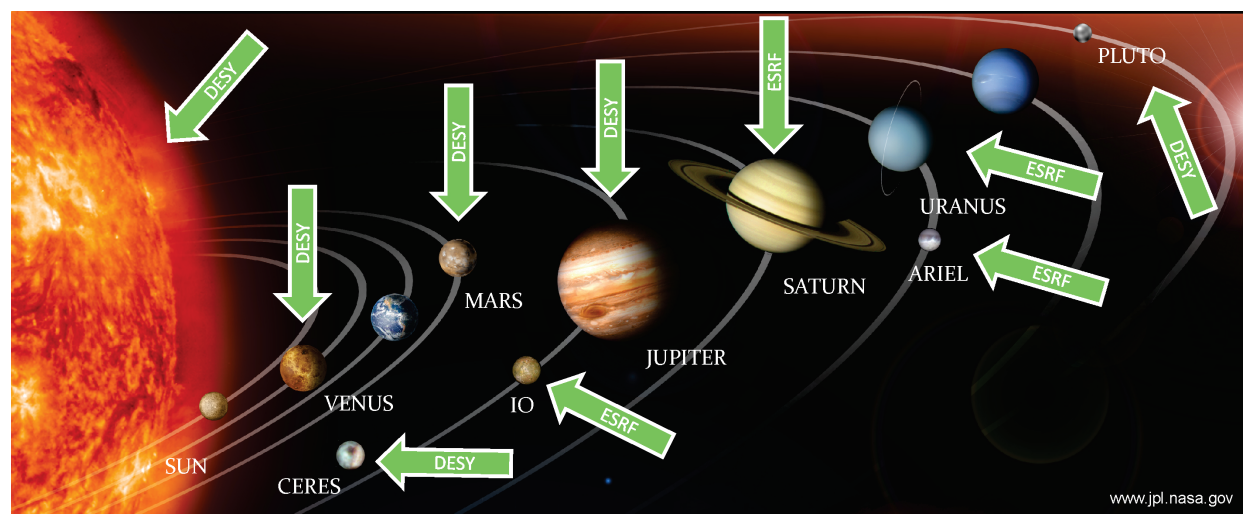

Figure 8: Overview over all samples utilized at synchrotron sources. The latter are identified by arrows.

In figure 8 all waveguide chips are shown, that have already been characterized at synchrotron sources. Starting with $S U N$ the chronological order of the chip fabrication is conform to the sequence of the objects in our solar system pointing away from its center. The chips named after moons were prepared directly after the corresponding planets. The sole exception from this order is CERES, which was prepared at last. The detailed list in table 8 also includes the chips that have not yet been utilized at any x-ray source, but are well-documented. For the characterized waveguides the chip length $l$, suited to the photon energy $E_{p h}$, the maximum transmission $T_{\text {max }}$, the channel geometry and the beamlines are itemized. At the x-ray source PETRA III at the Deutsches Elektronen-Synchrotron (DESY), the Institute for X-ray Physics hosts the GINIX (Göttingen Instrument for Nano-Imaging with X-rays) endstation at the P10 beamline, where many of the waveguides were introduced into the beam, the P03 beamline is also located at DESY. The other samples were taken to the European Synchrotron Radiation Facility (ESRF) to the beamlines BM20, ID01 and ID11. 


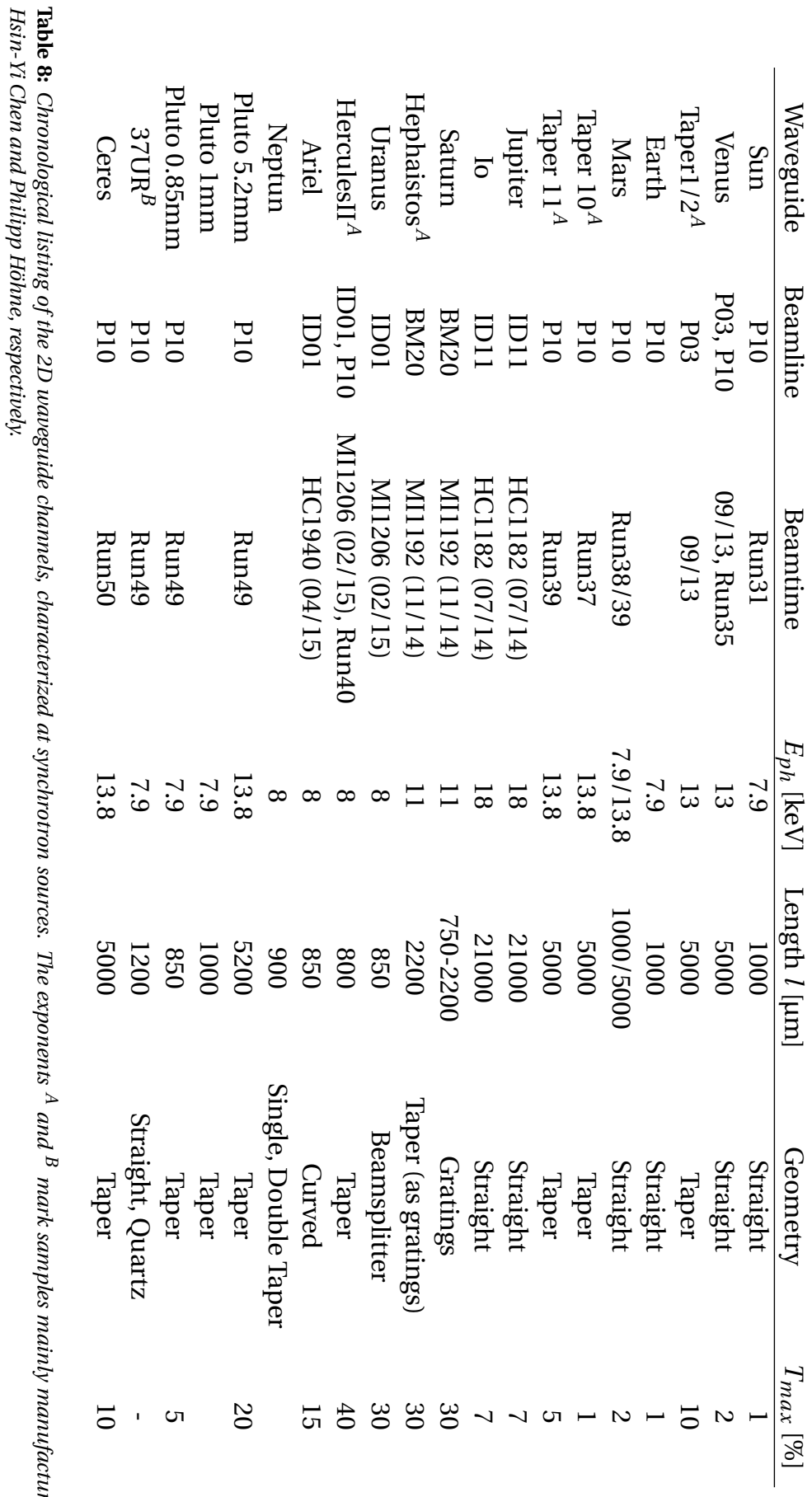


Apart from the geometry listed in table 8 . every chip carries an array of straight channels with widths in the range of $50 \mu \mathrm{m}-50 \mathrm{~nm}$, serving for aligning the chip with respect to the incoming radiation (see a comprehensive description in [46] p. 101-107).

An example for a documentation sheet is shown in figure 9. Here, additionally to the geometric dimensions and positions of the individual channels, the orientation of the chip referring to the mounting system at the beamline is depicted.

\section{Pluto5.2mm (15M)} through mic

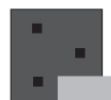

....

through oav

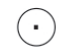

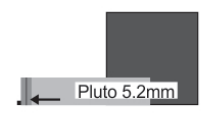
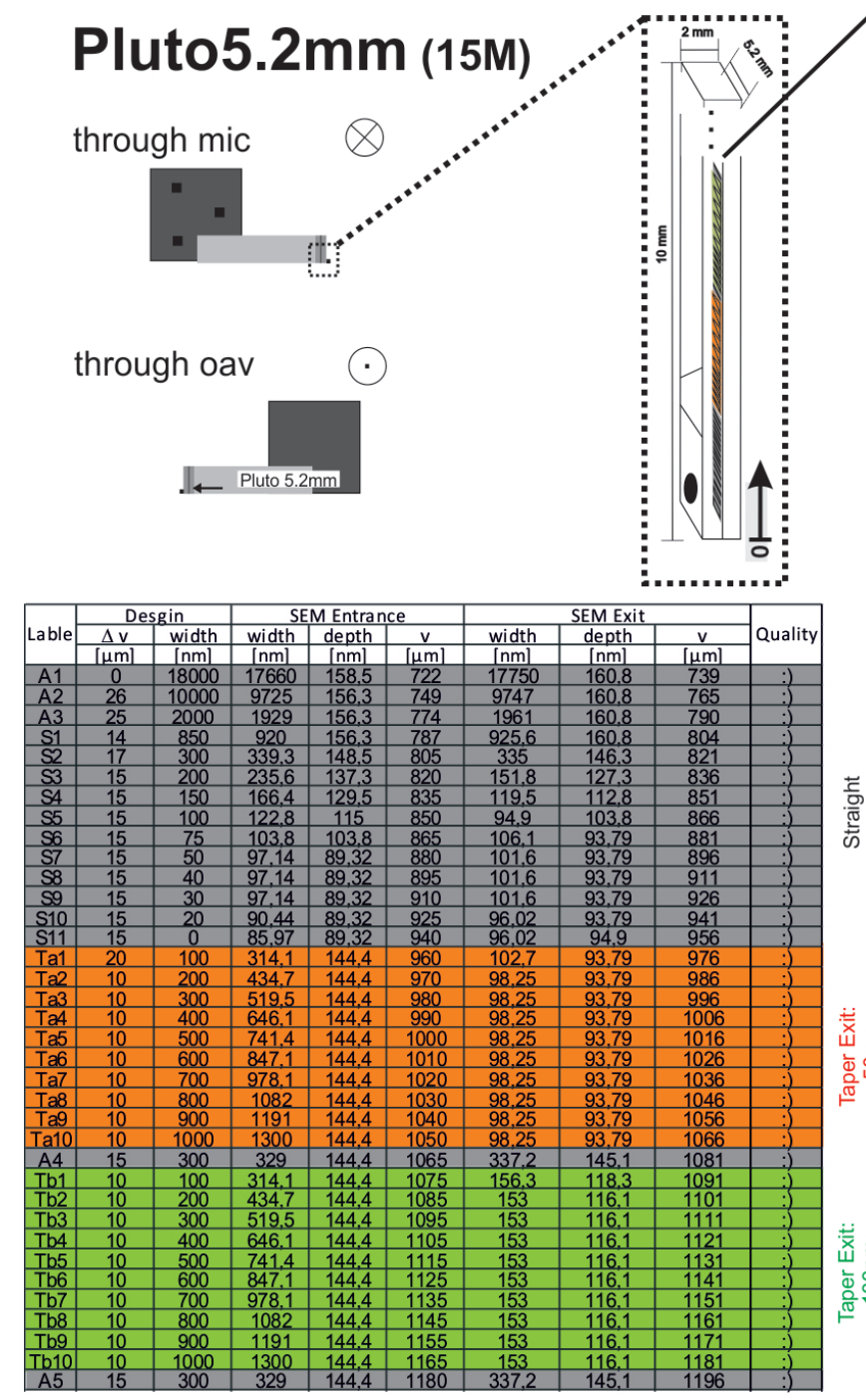

Figure 9: Documentation sheet of a waveguide chip. 



\section{Appendix B}

\section{PyPropagate source code}

In this section Python notebooks [53] are collected, covering various geometry types (straight, grating, taper, splitter, delay-lines and curvatures) and analysis tools. Apart from varying the angle of the incident wave field or the specific geometry parameters, several tools are implemented: A Fourier transformation to gain access to the mode occupation, the calculation of the Poynting vector to visualize the energy flux, a phase unwrapper to identify and eliminate ambiguities in the phase, a module to detect the position of the modes inside a channel and a free-space propagator to simulate the wave field in the near-field regime behind the channels. Additionally, two complement concepts are presented to circumvent the limits of the paraxial wave equation. For one thing a gradient is added to the index of refraction (see section 1.2.4 and for another the coordinate system is rotating during propagation. 


\title{
Straight channel analysis
}

\author{
In [1]: from pypropagate import * \\ \%matplotlib inline \\ import matplotlib.pyplot as plt \\ from matplotlib.colors import LogNorm \\ import math \\ from pypropagate.colormaps import fire_colormap
}

Create the settings object that stores simulation parameters.

In [2]: settings = presets.settings.create_paraxial_wave_equation_settings()

$s=$ settings. symbols

presets.boundaries.set_plane_wave_initial_conditions(settings)

s. u_boundary $=0$

settings.wave equation. set energy ( $7.9 *$ units.keV)

n_Si = presets.medium.create_material("Si", settings)

$n_{-}$Va $=1$

Define all variables for the waveguide geometry

In [3]: $w g$ = settings.create_category ('waveguide')

Prefocus $=w g$. create_symbol ("Prefocus",pc.Symbol);

wg. Prefocus $=0 *$ units.um;

Nachlauf = wg.create_symbol("Nachlauf",pc.Symbol);

wg. Nachlauf $=0 *$ units. um;

l_waveguide = wg.create_symbol ("l_waveguide",pc.Symbol);

wg. l_waveguide $=1000 *$ units.um;

\#Parameter for variation

wg. create symbol ("r waveguide",pc.Symbol);

$r_{\text {_waveguide }}=$ pc.parameter ( $r_{\text {__waveguide" }}, 0.05$ )

wg.r_waveguide $=r_{\text {_waveguide*units.um; }}$

wg.create_symbol ("incid_angle",pc.symbol);

incid_ang $\bar{l}=$ = pc.parameter ("incid_angle", 0.000 )

wg.incid_angle = incid_angle*units.degrees;

\#Angle of incomming field can be varied

s. $u \Theta=p c . \exp \left(1 j * s . k^{*} p c . \sin \left(w g . i n c i d \_a n g l e\right) * s . x\right)$

Define the simulation box size in physical units and in voxels.

In [4]: settings.simulation_box.set((2.0*units.um, 2*units.um, 1.0*units.mm), (1001, 10 $01,1000)$ ) 
Import important symbols into the local namespace.

In [5]: print "Importing: \%s" \%",".join(sorted(settings.symbols.names())) settings. symbols.export(locals())

Importing: $C, E, N x, N y, N z, d x, d y, d z, k, n$, omega, ra, $r c, r f, s x, s y, s z, u, u \theta, u \_b o u n d a r$ $y$, wavelength , $x, x i, x \max , x \min , y, y \circ, y i, y \max , y \min , z, z i, z \max , z \min$

Define the index as refraction as a waveguide in vacuum.

In [6]: inside_condition_square $=\left(\operatorname{abs}(x) * * 2<=w g \cdot r \_w a v e g u i d e * * 2\right) \&(a b s(y) * * 2<=$

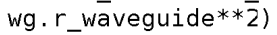

inside_waveguide $=p c .0 r(p c$. And (inside_condition_square), $z>=w g$. l_waveguide+ wg.Prefocus, $z<=w g$. Prefocus)

s.n = pc.piecewise (

Plot of the index of refraction for review.

In [7]: plot_settings = settings. $\operatorname{copy}()$

plot settings. simulation box. $\mathrm{Nx}=1000$

plot_settings.simulation_box.Nz $=1000$

$\mathrm{n}=\mathrm{s} \cdot \mathrm{n} \cdot$ function;

fig, $(\operatorname{ax} 1, a \times 2)=p l t$. subplots $(1,2$, figsize= $(10,5))$;

plot $(n(s . x, 0, s . z)$, plot_settings, ax = ax 1$)$;

plot $(n(s . x, s . y, 0.5 *$ units. $m$ m), plot_settings, $a x=a x 2)$;

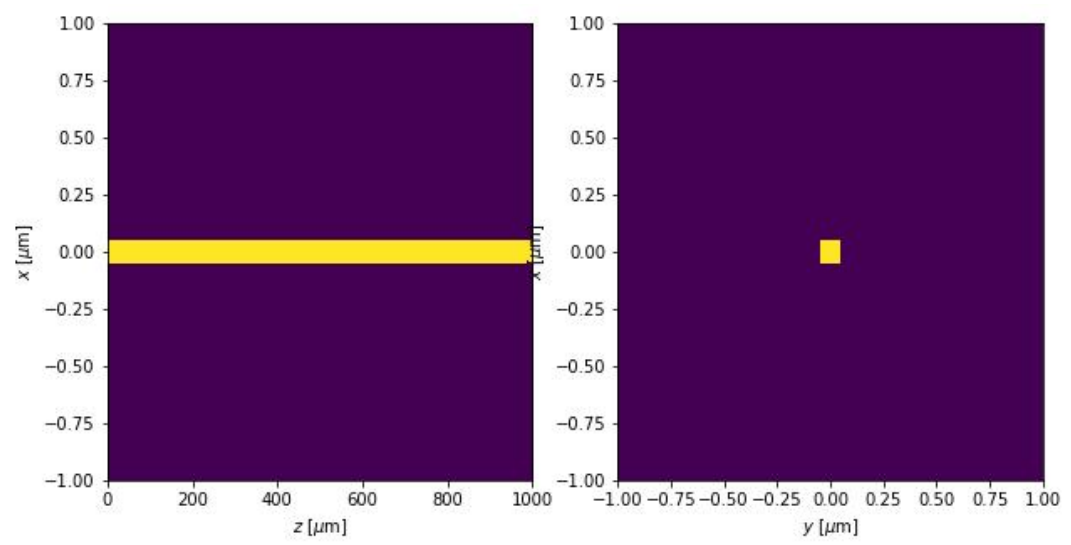


In [14]: \# Angle variation

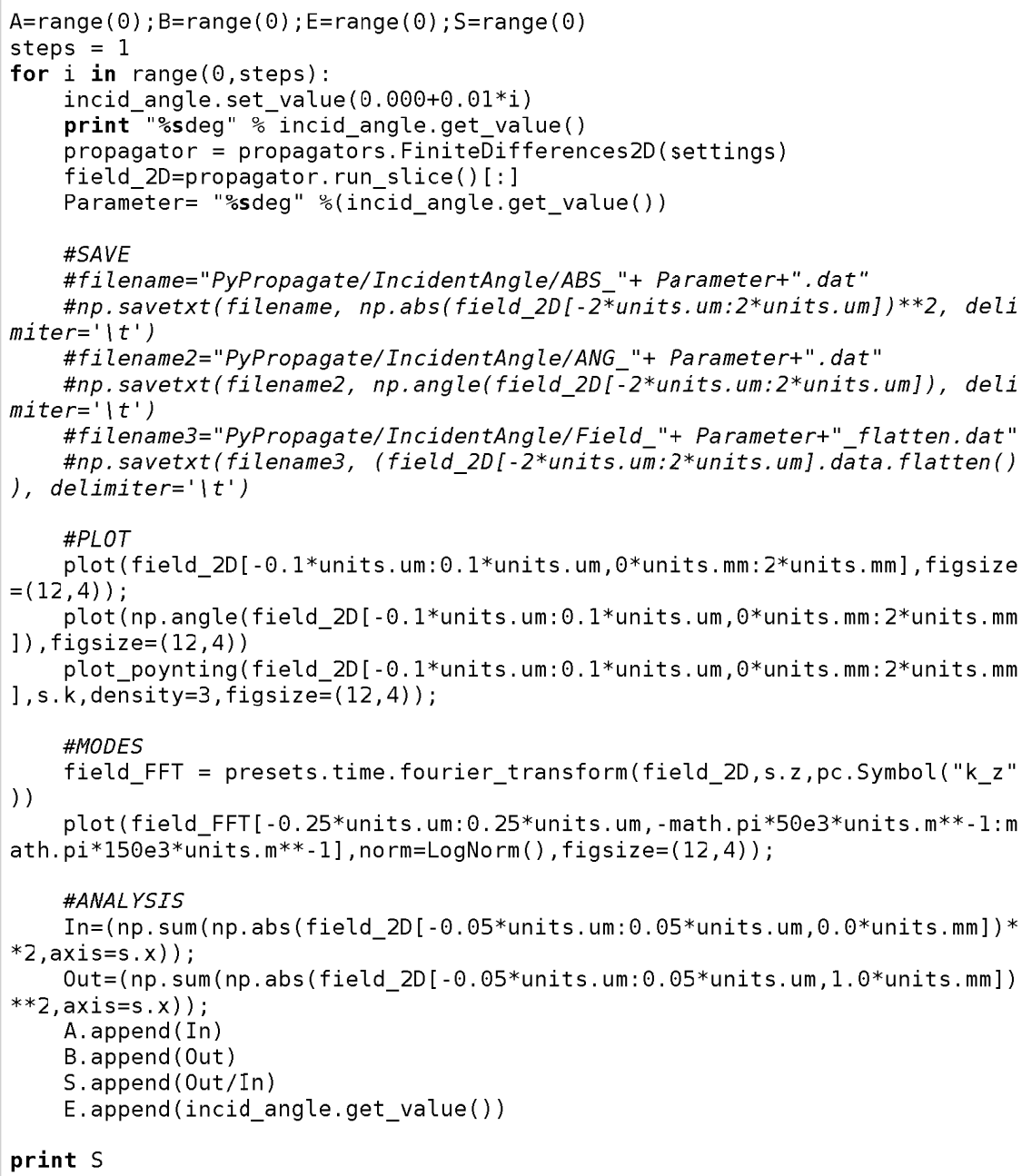


O. Odeg

propagating:

$999 / 999[0.3 s<0(0) s]]$
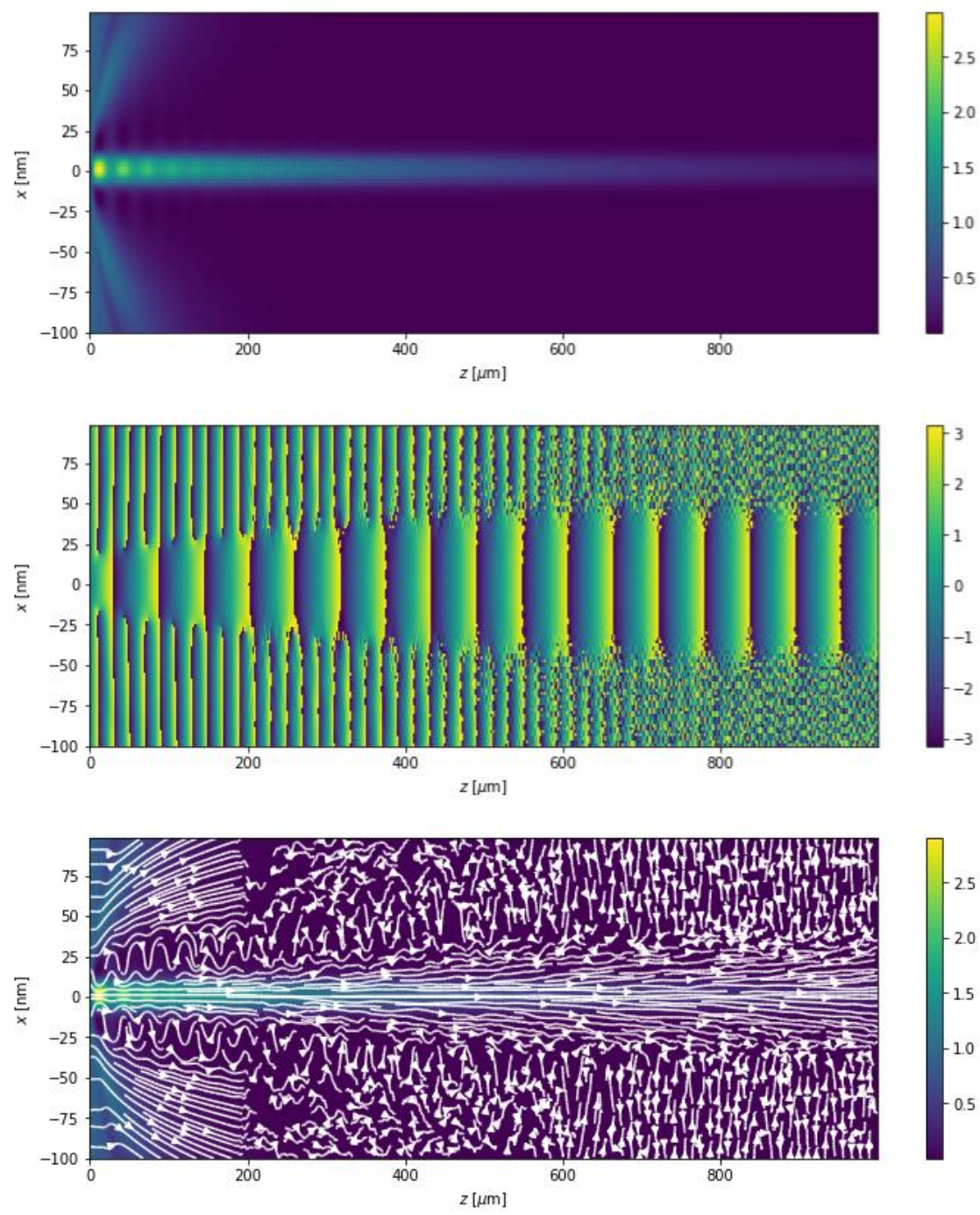


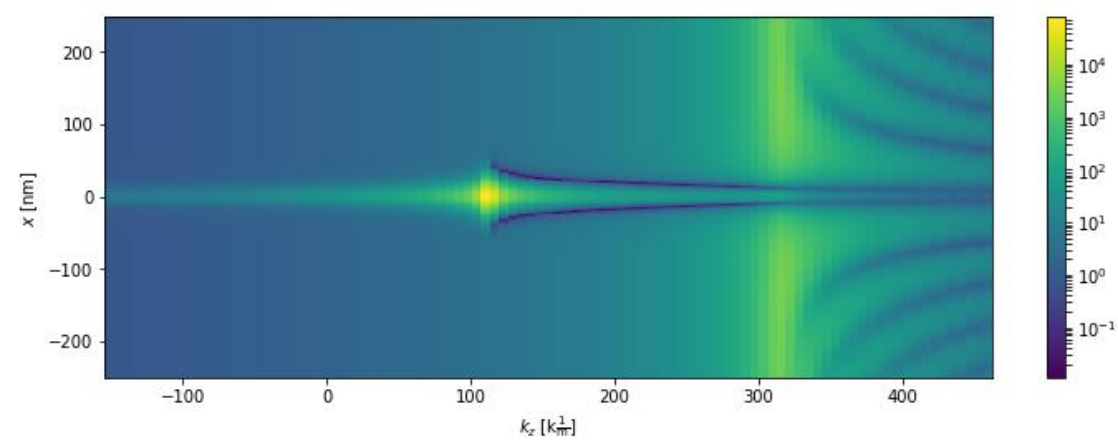

$[0.038269072119256113]$

\section{Phaseunwrapping}

In [9]: from skimage.restoration import unwrap_phase

fig, $(a \times 1, a \times 2)=$ plt. $\operatorname{subplots}(1,2$, figsize $=(15,5))$;

plot (unwrap_phase (np.angle (field_2D [-0.1*units.um:0.1*units.um, $0 *$ units.mm: 2 *units.mm])), ax = axl);

plot ( (np.angle(field_2D [-0.1*units.um:0.1*units.um, $0^{*}$ units.mm: $2 *$ units.mm])) , $a x=a x 2)$;
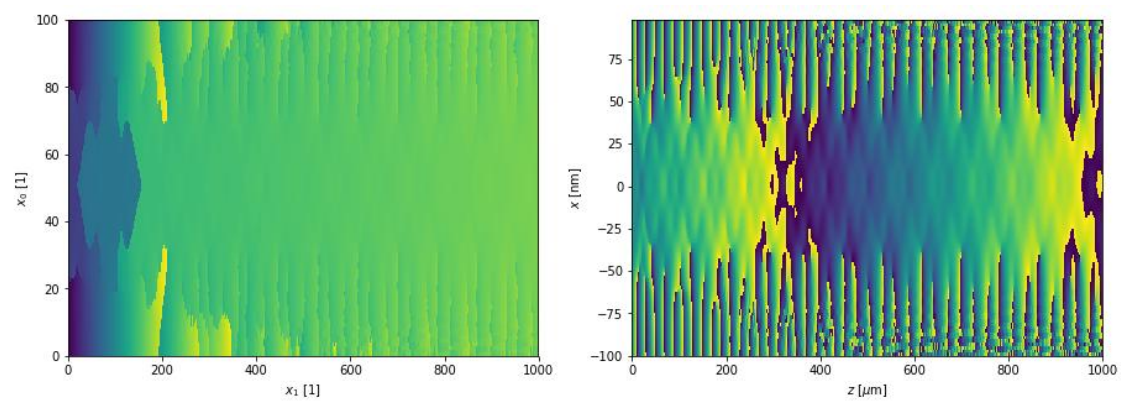
In [17]: \# Width variation

$E=\operatorname{range}(0) ; \operatorname{Max} 1=\operatorname{range}(0) ; \operatorname{Max} 2=\operatorname{range}(0) ; \operatorname{Max} 3=\operatorname{range}(0) ; \operatorname{Min} 1=\operatorname{range}(0) ; \operatorname{Min} 2=r$ ange $(0) ;$ Min $3=$ range $(0)$

steps $=1$

for $i$ in range $(0$, steps):

r_waveguide.set_value $(0.010+0.005 * i)$

print "\%sum" \% $\bar{r}$ waveguide.get_value()

propagator $=$ propagators. FinitēDifferences $2 \mathrm{D}$ (settings)

field_2D=propagator.run_slice() [: ]

Radius $=$ "osum" \%(r_waveguide.get_value ()$)$

E.append (r_waveguide.get_value( ) )

\#PLOT

plot(field_2D[-0.005*units.um:0.15*units.um, $0 *$ units.mm: $0.1 *$ units.mm $], f i g$ size $=(10,3))$;

plot (field_2D [-0*units.um, $0 *$ units.mm: $0.1 *$ units.mm $] * 100$, figsize $=(8,3)$, tit le $=$ "Intensity at $\$ \mathrm{x}=0 \$$ ")

plot (np.angle (field_2D [-0.005*units.um: $0.15^{*}$ units.um, $0 *$ units.mm: 0.1 *unit s.mm ]), figsize $=(10,3))$

\#FIND MAXIMA 1

a=abs (field_2D [- $0.005 *$ units.um: $\odot .15^{*}$ units.um, $0: 30 *$ units.um $]$ )**2;

$\mathrm{b}=\mathrm{np}$. where $(\overline{\mathrm{a}}==\mathrm{a} \cdot \max ()) ; \max 1=\max (\mathrm{b}[1]) ; \operatorname{Max} 1$. append $(\max 1)$

\#FIND Minima 1

al=abs (field_2D [0*units. um, max $]^{*}$ units. um+2*units. um : $2^{*}$ max $1 *$ units. um+10*u nits. um ])**2;

$\mathrm{b} 1=\mathrm{np}$. where $(\mathrm{a} 1==\mathrm{a} 1 . \min ()) ; \min 1=\min (\mathrm{b} 1[0])+\max 1+2 ; \quad \operatorname{Min} 1 . \operatorname{append}(\min 1)$ \#FIND MAXIMA 2

c=abs (field_2D [-0.005*units.um: $0.15^{*}$ units.um, minl*units. um: $2^{*}$ min $1^{*}$ units. um+10*units. um] $) * * 2$;

$\mathrm{d}=\mathrm{np}$. where $(\mathrm{c}=\mathrm{c} \cdot \max ()) ; \quad \max 2=(\min 1)+\max (\mathrm{d}[1]) ; \quad \operatorname{Max} 2 \cdot \operatorname{append}(\max 2)$

\#FIND Minima 2

$\mathrm{cl}=\operatorname{abs}\left(\mathrm{field} \_2 \mathrm{D}\left[0^{*}\right.\right.$ units.um, max $2 *$ units.um+2*units.um : ( (max $\left.\left.2-\max 1\right)+\max 2\right) * u$ nits. um ]) $* * 2$;

$\mathrm{d} 1=\mathrm{np}$. where $(\mathrm{c} 1==\mathrm{cl} \cdot \min ()) ; \quad \min 2=\min (\mathrm{d} 1[0])+\max 2+2 ; \quad \operatorname{Min} 2 \cdot \operatorname{append}(\min 2)$ \#FIND MAXIMA 3

$f=a b s$ (field_2D [- $0.005^{*}$ units.um: $\odot .15^{*}$ units.um, (min2*units.um) : (min $\left.1+m i n 2\right)$ *units. um ]) **2;

$g=n p \cdot$ where $(f==f \cdot \max ()) ; \quad \max 3=(\min 2)+\max (g[1]) ; \quad \operatorname{Max} 3 . \operatorname{append}(\max 3)$

\#FIND Minima 3

$\mathrm{fl} 1=$ abs ( field_2D [0*units.um, max $3^{*}$ units. um+2*units. um : ( (max $\left.\left.2-\max 1\right)+\max 3\right) * u$ nits.um]) $* * 2$;

$\mathrm{gl}=\mathrm{np} \cdot$ where $(\mathrm{fl}==\mathrm{fl} \cdot \min ()) ; \quad \min 3=\min (\mathrm{g} 1[0])+\max 3+2 ; \quad \operatorname{Min} 3 . \operatorname{append}(\min 3)$

$E n=\left[1 \% .3 f^{\prime} \%\right.$ elem for elem in $\left.E\right]$

print "Radius [um]: ", En

print "Maxl [um]: ", Max 1

print "Max2 [um]: ", Max2

print "Max3 [um]: ", Max3

print "Minl [um]: ", Min]

print "Min2 [um]: ", Min2

print "Min3 [um]: ", Min3 


\subsection{1 um}

propagating:|

$999 / 999[0.3 \mathrm{~s}<0(0) \mathrm{s}]]$
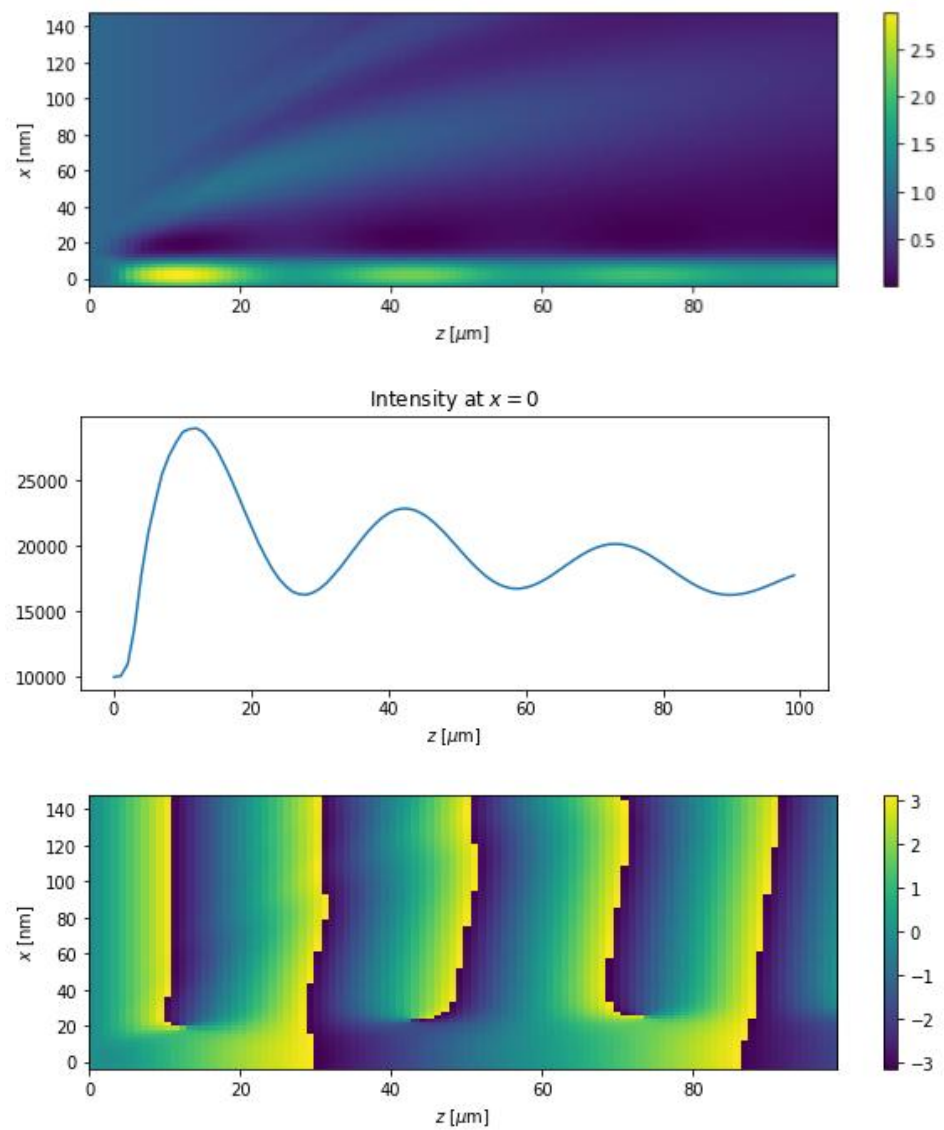

Radius [um]: ['0.010']

Max 1 [um ]: [12]

Max2 [um]: [42]

Max3 [um]: [73]

Min 1 [um]: [28]

Min2 [um]: [59]

Min3 [um ]: [90] 
In [107]: solver = propagators. FiniteDifferences3D (settings)

field_3D = solver.run_slice() $[:,:,:]$

plot (field_3D[-0.05*units.um:0.05*units.um, 0,0*units.mm: $2 *$ units.mm ], figsize $=(8,3))$;

0.03 um

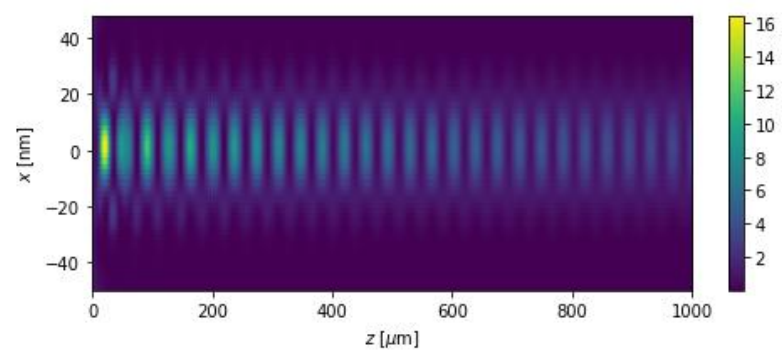

\section{Free Space Propagation}

In [52]: input_field $=$ field_3D [-0.1*units.um: $0.1^{*}$ units.um, $-0.1^{*}$ units.um: $0.1 *$ units.u $\mathrm{m}, 1^{*}$ units, $\left.\mathrm{mm}\right]$

In [53]: vacuum settings = presets. settings.create next settings (settings)

presets. boundaries.set_initial (vacuum_settings,presets.boundaries.add_paddi ng (input_field,5))

vacuum_settings.wave_equation. $n=1$

vacuum settings. simulation box. sz $=500 *$ units.um

vacuum_settings.simulation_box.Nz $=101$

In [54]: propagator = propagators.Fresnel3D (vacuum_settings)

nearfield= propagator,run_slice() [:, : , : ]

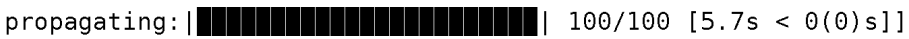

In [108]: $f i g,(a x 1, a \times 2)=p l t . \operatorname{subplots}(1,2, f i g s i z e=(8,3))$;

$\operatorname{plot}\left((\right.$ nearfield $[:, 0,:])$, norm $=\operatorname{LogNorm}\left(\operatorname{vmin}=10^{* *}-6, \quad \operatorname{vmax}=20\right), \quad a x=a x 1, \mathrm{cmap}=\mathrm{f}$ ire_colormap()); plot ((input_field), ax = ax2,cmap=fire_colormap());
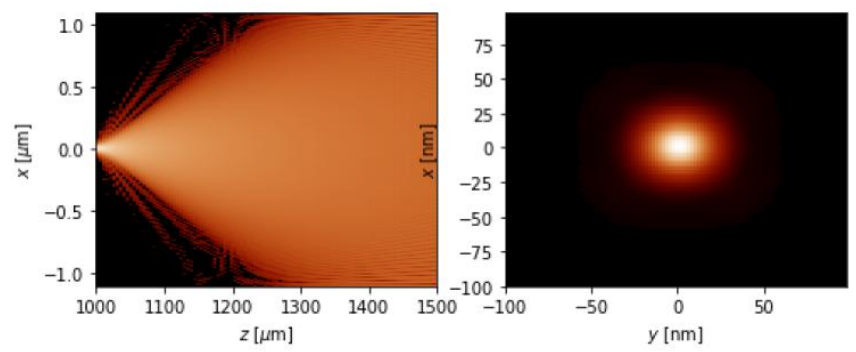


\section{Grating}

In [13]: wg.create_symbol("Spacing",pc.Symbol);

Spacing = pc.parameter ("Spacing",0.3)

wg. Spacing = Spacing*units.um;

Define the index as refraction as a waveguide in vacuum.

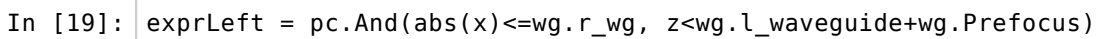

exprRight $=p c \cdot 0 r\left(z>=w g . l_{-}\right.$waveguide+wg.Préfocus, $z<=w g$.Prefocus $)$

exprStraightTop5 = pc.And $(z<=w g$. l_waveguide+wg.Prefocus, abs $(y)<=w g \cdot r$ wg , $x>=5 * w g$.Spacing-wg. $r \_w g, x<=5 * w g \cdot$ Späing+wg. $\left.r \_w g\right)$

exprStraightTop4 $=$ pc. And $(z<=w g$. l waveguide+wg.Prefocus, abs $(y)<=w g . r . w g$, $x>=4 * w g$. Spacing-wg. $r \_w g, x<=4 * w g \cdot$ Späcing+wg.r_wg)

exprStraightTop3 $3=p c$. And $(z<=w g$. l waveguide $+w g$.Prefocus, abs $(y)<=w g . r$ wg, $x>=3 * w g$. Spacing-wg.r_wg,$x<=3 * w g$. Späcing+wg.r_wg)

exprStraightTop2 $=$ pc.And $(z<=w g$. l_waveguide $+w g$.Prefocus, abs $(y)<=w g \cdot r$ wg $x>=2 * w g$.Spacing-wg. $r \_w g, x<=2 * w g$. Spacing+wg. $\left.r \_w g\right)$

exprStraightTop1 $1=p c$. And $\left(z<=w g\right.$. l_waveguide+wg.Prefocus, abs $(y)<=w g \cdot r \_w g$, $x>=1 * w g$. Spacing-wg. $r \_w g, x<=1 * w g$. Spacing+wg. $\left.r \_w g\right)$

exprStraightBottom $1=p c$. And $\left(z<=w g . l\right.$ waveguide+wg.Prefocus, abs $(y)<=w g . r \_w g$, $x>=1 *(-w g$. Spacing $)-w g \cdot r \cdot w g, x<=1 *(-w g$. Spacing $)+w g \cdot r \cdot w g)$

exprStraightBottom $2=p c \cdot$. And $\left(z<=w g\right.$. l_waveguide+wg.Préfocus, abs $(y)<=w g . r \_w g$, $x>=2 *(-w g$. Spacing $)-w g \cdot r \_w g, x<=2 *(-w g$.Spacing $\left.)+w g \cdot r \_w g\right)$

exprStraightBottom3 $=$ pc. And $\left(z<=w g\right.$. l_waveguide+wg.Préfocus, abs $(y)<=w g . r \_w g$, $x>=3^{*}(-w g \cdot$ Spacing $)-w g \cdot r+w g, x<=3 *(-w g \cdot$ Spacing $\left.)+w g \cdot r+w g\right)$

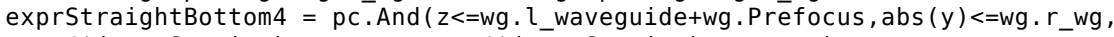
$x>=4 *(-w g$. Spacing $)-w g \cdot r \_w g, x<=4 *(-w g$. Spacing $\left.)+w g \cdot r+w g\right)$

exprStraightBottom5 = pc. And $\left(z<=w g\right.$. l_waveguide+wg.Préfocus, abs $(y)<=w g . r \_w g$, $x>=5 *(-w g$. Spacing $)-w g \cdot r \_w g, x<=5 *(-w g \cdot$ Spacing $\left.)+w g \cdot r \_w g\right)$

inside waveguide $=p c .0 r(p c$. And $($ exprLeft, abs $(y)<=w g \cdot r$ wg), exprRight, exprS traightTop1, exprStraightTop2, exprStraightTop3, exprStraightTop4, exprStraight Top5, exprStraightBottom1, exprStraightBottom2, exprStraightBottom3, exprStraig htBottom4, exprStraightBottom5)

s.n = pc.piecewise ( $n \_V a$, inside_waveguide $\left.),\left(n \_S i, T r u e\right)\right)$

Plot of the index of refraction for review. 
In [20]: $n=s . n . f u n c t i o n ; f i g,(a x 1, a \times 2)=p l t . \operatorname{subplots}(1,2, f i g s i z e=(10,4))$;

plot $(n(s . x, 0, s . z)$, settings, $a x=a x 1) ; \operatorname{plot}(n(s . x, s \cdot y, 0.5 *$ units.mm), settin gs, $a x=a \times 2)$;
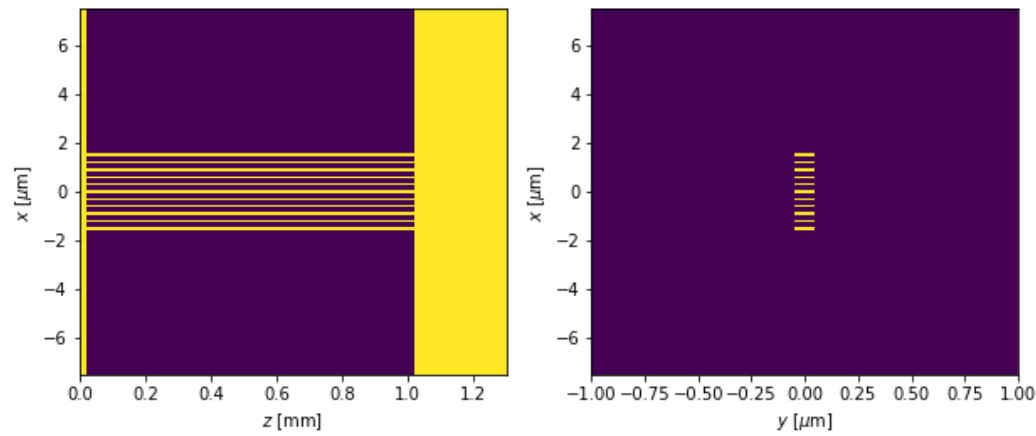

In [22]: propagator = propagators.FiniteDifferences2D(settings)

field $2 D=$ propagator. run slice () [: ]

plot ( (field_2D [-4*units.um:4*units.um, : ]), figsize $=(12,4)) ;$ plot (np.angle $(f i e$ ld 2D[-4*units.um:4*units.um, : ]),figsize=(12,4));

propagating:

$1299 / 1299[2.6 s<0(0) s]]$
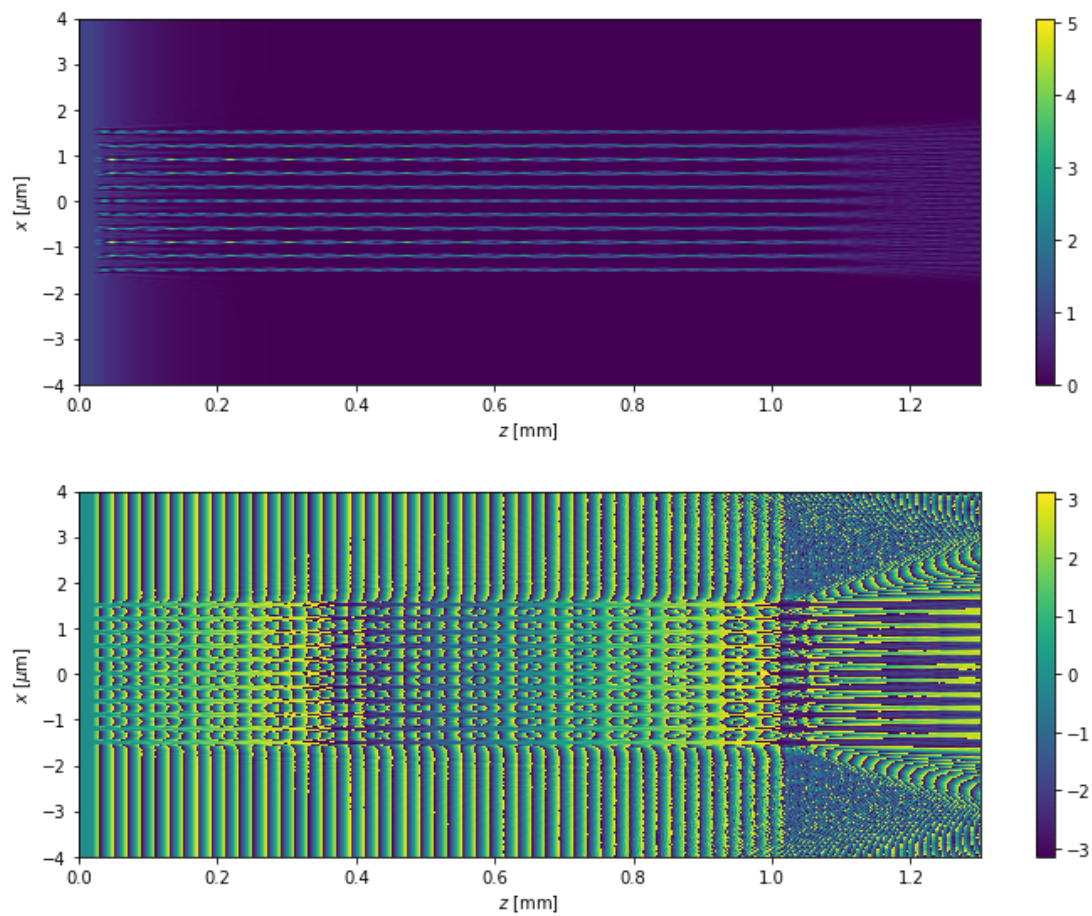


\section{Taper}

In [13]: alpha = wg.create_symbol ("alpha",pc.Symbol);

Prefocus $=w g$. create_symbol ("Prefocus",pc.Symbol);

\#Parameter for Variation

wg.create symbol ("Entrance",pc.Symbol):

Entrance $=$ pc.parameter ("Entrance" 1.5 )

wg. Entrance $=$ Entrance*units.um;

Define the index as refraction as a waveguide in vacuum.

In [17]: wg.alpha=pc.atan(( (wg.Entrance-wg.Exit)/2)/wg. l_waveguide); $w g \cdot W G R=((w g$. Entrance $-w g . E x i t) / 2)-p c \cdot \tan (w g \cdot a l p h \bar{h}) *(z-w g$. Prefocus $)$;

inside condition ellipse $=(x * * 2+y * * 2)<=$ wg.WGR**2

inside_condition_square $=p c \cdot 0 r(p c \cdot 0 r(p c \cdot \operatorname{And}(\operatorname{abs}(y)<=w g$. WGR, abs $(x)<=w g$. WGR $)$

,$(\operatorname{abs}(\bar{x})<=w g \cdot r$ waveguide) \& (abs $(y)<=w g \cdot r$ waveguide), ( $z>=w g$. Prefocus $+w$ g. l_waveguide+wg. Nachlauf)) , $z<=w g$. Prefocus)

$\mathrm{s} \cdot \mathrm{n}=\mathrm{pc} \cdot \mathrm{piecewise}\left(\left(\mathrm{n} \_\right.\right.$Va, inside_condition_square $\left.),\left(\mathrm{n} \_\mathrm{Si}, \mathrm{True}\right)\right)$

Plot of the index of refraction for review.

In [18]: $\mathrm{n}=\mathrm{s} . \mathrm{n}$. function;

fig, $(a \times 1, a \times 2)=p l t$. $\operatorname{subplots}(1,2, f i g s i z e=(10,4))$;

$p \operatorname{lot}(n(s . x, 0, s . z)$, settings, $a x=a x 1) ; p \operatorname{lot}(n(s \cdot x, s \cdot y, 1$ *units.mm), settings, $\mathrm{ax}=\mathrm{ax} 2$ );
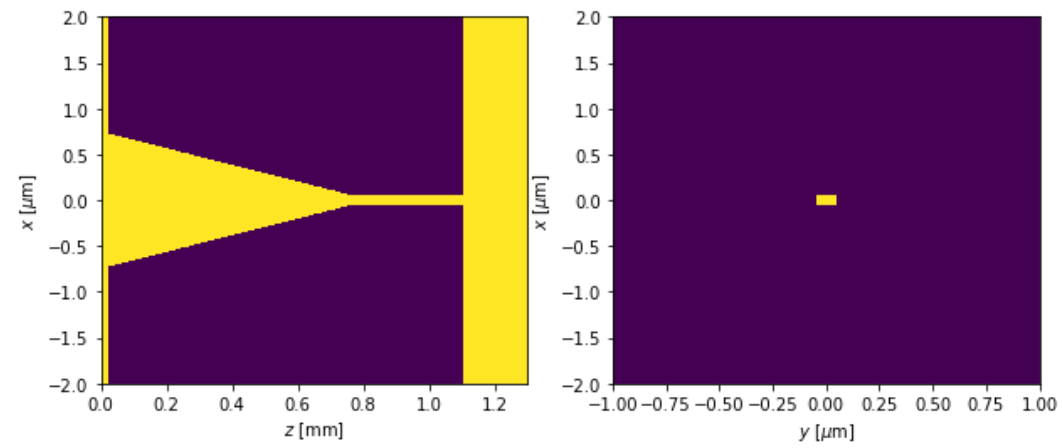

\section{1+1D simulation}

In [19]: propagator $=$ propagators.FiniteDifferences $2 \mathrm{D}$ (settings)

$A=\operatorname{range}(\theta) ; E=\operatorname{range}(\theta) ; C=\operatorname{range}(\theta) ; D=\operatorname{range}(\theta) ; W=\operatorname{range}(\theta) ; F=\operatorname{range}(\theta)$ 
In [20]: steps $=2$

for $i$ in range $(\theta$, steps):

Entrance.set value $(0.4+0.5 * i)$

print "\%sum" \% Entrance.get_value()

field $2 D=$ propagator. run slice( $)[-2 *$ units.um: $2 *$ units.um]

Parameter $=$ "\%sum" \%(Entrance.get_value ()$)$

plot (np.abs (field_2D [ - $1 *$ units.um: $1 *$ units.um]) $* * 2, f i g s i z e=(10,3))$;

$\mathrm{P}=\mathrm{np} . \operatorname{amax}(\mathrm{np} . \operatorname{amax}(\mathrm{np}$. abs (field 2D [ -5*units.nm:5*units.nm, 1 *units.mm: 1.3 *units.mm])**2, axis=s.x));

In $=(n p . \operatorname{sum}(n p$. abs $($ field_2D [-50*units.nm:50*units.nm,Prefocus $]) * * 2$, axis= S.x)) ;

Out $=(n p . \operatorname{sum}(n p$. abs (field_2D [-50*units.nm:50*units.nm,wg.Prefocus+wg.l_w aveguide] ) $* * 2$, axis $=$ s. $x)$ ) ;
A. append $(P)$
E. append (Entrance.get value())
\# maximum Exit-Intensity
C. append (In)
D. append (Out)
F.append (Out/(In)*100) \# Gain relative to a straight WG

print $A$

print $F$

๑. 4 um

propagating:

$1299 / 1299[0.8 \mathrm{~s}<0(0) \mathrm{s}]]$

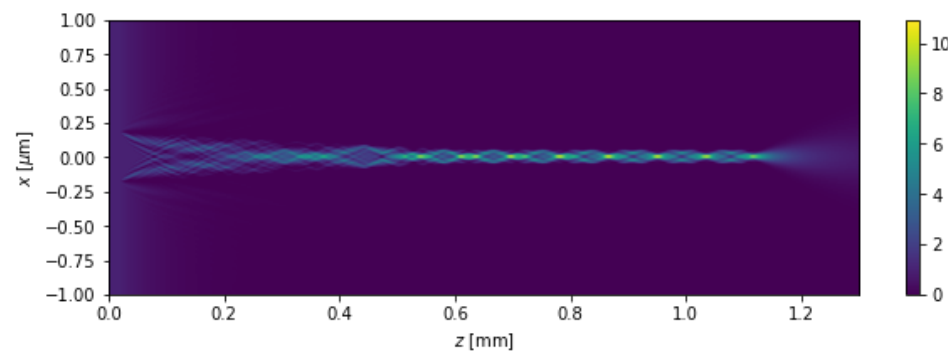

0.9 um

propagating:

$1299 / 1299[0.7 \mathrm{~s}<0(0) \mathrm{s}]]$

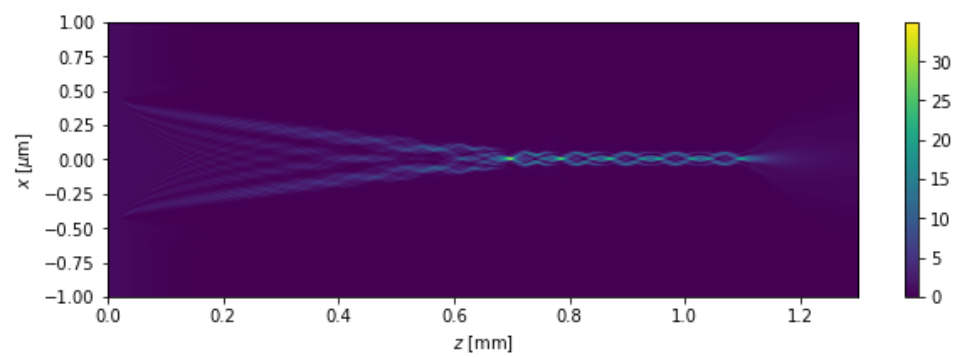

[10.40636316827916, 23.936939734715054]

[316.37876689555389, 637.69636408170209] 


\section{Splitter}

In [3]: l_splitMin=wg.create_symbol("l_splitMin", 0 *units.um);

l_splitMax=wg.create_symbol("l_splitMax", 0 *units.um);

Deltax splitMax = wg.create symbol ("Deltax splitMax", 1.387*units.um);

Deltax_splitMin = wg.create_symbol("Deltax_splitMin", $-1.387 *$ units.um);

Deltax split=wg.create_symbol("Deltax_split", info="x Position where Split $h$ appens");

l split=wg.create symbol("l split");

tan_alpha=wg.create_symbol ("tan_alpha") ;

wg.create_symbol("alpha_split",pc.Symbol);

alpha_splìt $=$ pc.parameter ("alpha_split",0.02)

wg.alpha_split $=$ alpha_split*units.

Define the index as refraction as a waveguide in vacuum.

In [6]: wg.Deltax split $=$ (wg.Deltax splitMax + wg.Deltax splitMin) $/ 2$

wg.l_split=(wg.l_splitMax+wg.l_splitMin)/2

wg.tan_alpha $=p \bar{c} \cdot \tan \left(w g \cdot a l p h a \_s p l i t\right)$

exprLeft $=p c$.And $\left(\right.$ abs $(x)<=w g . r$ waveguide, $\left.z<l \_s p l i t\right)$

exprMidTop $=p c$. And $(x-w g$. Deltax split $>=w g \cdot \tan a l p h a *(z-w g . l$ split-wg.Prefoc us) -wg.r_waveguide*pc.sqrt(1+wg.tan_alpha**2)+wg.r_waveguide, $x-w g$. Deltax_s plit<=wg.tan_alpha*(z-wg.l_split-wg.Prefocus) $+w g . r$ waveguide*pc.sqrt $(1+w g . t$ an_alpha**2)+wg.r_waveguide)

exprMidBottom $=p \bar{c}$. And $(x-w g$. Deltax split $>=-w g$.tan alpha* $(z-w g . l$ split-wg.Pr efocus) -wg.r_waveguide*pc.sqrt(1+wg.tan_alpha**2)-wg.r_waveguide, $x-w g$. Delt ax_split< $<-w \bar{g} \cdot \tan$ alpha* $(z-w g$. l_split $-w \bar{g}$. Prefocus $)+w g \cdot \bar{r}$ waveguide*pc.sqrt ( 1 + wg.tan_alpha**2)-wg.r_waveguide)

exprMid $=$ pc.0r(pc. And (exprMidTop, $z>=w g . l$ split, $z<=w g . l$ waveguide+wg.Pref ocus), pc.And (exprMidBottom, $z>=w g . l \_s p l i t, z<=w g . l$ waveguide+wg.Prefocus)) exprRight $=p c \cdot 0 r(z>=w g . l$ waveguide+wg.Prefocus, $z<=\overline{w g}$.Prefocus $)$

inside_waveguide $=p c .0 r(p c$. And $(p c .0 r($ exprLeft, exprMid), abs $(y)<=w g . r$ wave guide), exprRight)\#Or(exprLeft, exprMid)\#, exprRight)

s.n = pc.piecewise ( $\left(n \_V a\right.$, inside_waveguide $),($ n_Si,True $\left.)\right)$

In [7]: $\mathrm{n}=\mathrm{s} . \mathrm{n}$. function; fig, $(\operatorname{ax} 1, \operatorname{ax} 2)=\operatorname{plt} . \operatorname{subplots}(1,2, \operatorname{figsize}=(8,3))$;

$\operatorname{plot}(\mathrm{n}(\mathrm{s} . \mathrm{x}, 0, \mathrm{~s} . \mathrm{z})$, settings, $a x=a x 1) ; \operatorname{plot}\left(\mathrm{n}\left(\mathrm{s} . \mathrm{x}, \mathrm{s} . \mathrm{y}, 0.8^{*}\right.\right.$ units.mm $)$, setting s, $a x=a \times 2)$;

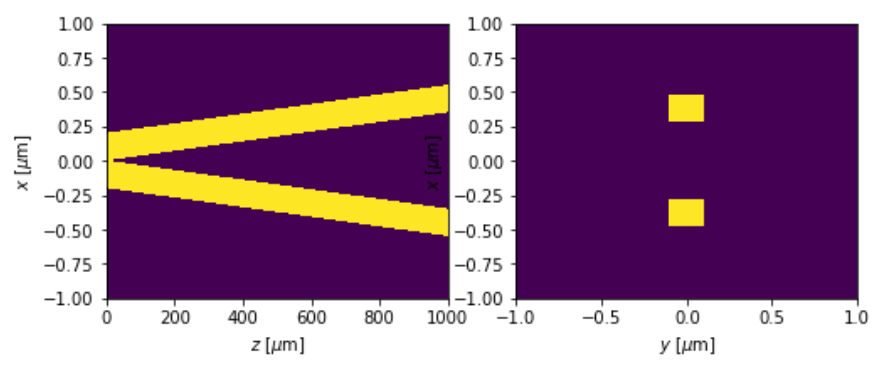




\section{1+1D simulation}

In [8]: propagator $=$ propagators.FiniteDifferences2D (settings)

$\mathrm{E}=\operatorname{range}(\theta) ; \mathrm{S}=\operatorname{range}(\theta) ; \mathrm{V}=\operatorname{range}(\theta)$

In [9]: steps $=1$

for $i$ in range $(0$, steps):

alpha_split.set_value $(0.02+0.01 * i)$

print ${ }^{-} \%$ sdeg" \% alpha_split.get value()

field_2D=propagator.run_slice() [-5*units.um: $5 *$ units.um ]

Variable= "\%sdeg" \%(alpha split.get value ()$* 1000)$

plot $($ field_2D,figsize $=(10,3))$;

In $=(n p . \operatorname{sum}(n p$. abs (field_2D [- $0.1 *$ units.um: $0.099 *$ units.um,Prefocus $]) * * 2, a$ $\mathrm{xis}=\mathrm{s} \cdot \mathrm{x}))$;

Out $=($ np.sum (np.abs (field_2D [ - 4.50*units.um: 4.5*units.um,wg.Prefocus+wg . l_waveguide] $) * * 2$, axis $=s \cdot x))$;

Distance $=($ np.tan $($ alpha split.get value ()$*($ math.pi $) / 180) * 1000 * 2)$

V.append(alpha_split.get_value())

S. append (Out/ $(\overline{2} * \operatorname{In}) * 100)$

E.append(Distance) \# Distance of exits

print $S$

print $E$

$\odot .02 \mathrm{deg}$

propagating:

$999 / 999[0.4 \mathrm{~s}<0(0) \mathrm{s}]]$

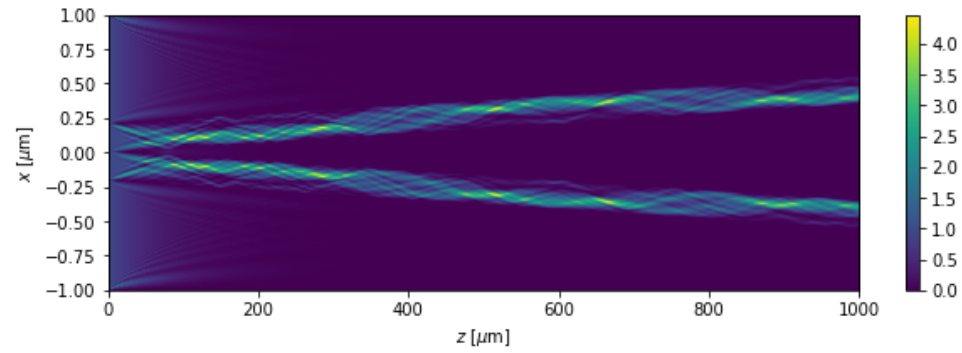

[96.45031078445821]

[0.69813172915281019]

\section{1+2D simulation}

In [56]: solver = propagators.FiniteDifferences3D (settings)

field_3D = solver.run_slice ()$[:,:,:]$

The full 3D animation field is stored as a global variable, delete it after usage to free memory on the host computer.

In [29]: \#del field_3D 


\section{Free Space Propagation}

In [55]: input_field = field_3D[-1*units.um: 1 *units.um,-0.1*units.um: $0.1 *$ units.um, 10 $\odot *$ units.um]

fig, $(\operatorname{ax} 1, a \times 2)=$ plt. $\operatorname{subplots}(1,2, \operatorname{figsize}=(15,5)) ;$

plot (field_3D $[:, 0,:], \quad a x=a x 1)$;

plot (input_field, $a x=a \times 2$ );
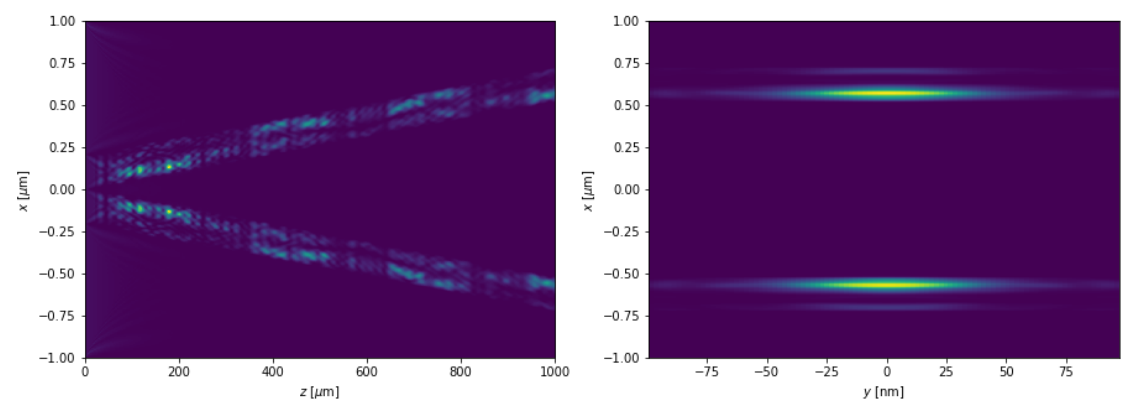

In [30]: vacuum_settings = presets.settings.create_next_settings (settings)

presets.boundaries.set_initial (vacuum_settings,presets.boundaries.add_paddi ng (input_field, 1.5))

vacuum_settings.wave_equation. $n=1$

vacuum_settings.simulation_box.sz $=500 *$ units.um

vacuum_settings.simulation_box.Nz $=100$

In [31]: propagator = propagators.Fresnel3D (vacuum_settings)

nearfield $=$ propagator.run_slice ()$[:,:,:]$

propagating:

$99 / 99[8.6 \mathrm{~s}<\theta(\theta) \mathrm{s}]]$ 
In [35]: $f i g,(a \times 1, a \times 2)=p l t . \operatorname{subplots}(1,2, f i g s i z e=(10,5))$;

plot (np. $\log 10$ (np.abs (nearfield [-1*units.um: $1 *$ units.um, $0,:]$ )**2), ax = ax $1, \mathrm{~cm}$ ap $=$ 'jet') ;

plot (np. $\log 10$ (np.abs (nearfield [-2*units.um: $2 *$ units.um, - $0.5 *$ units.um: $0.5 *$ uni ts.um, 5*units.um+wg. l waveguide])**2), ax = ax2);

fig, $(a \times 1, a \times 2)=$ plt.subplots $(1,2$, figsize $=(10,5))$;

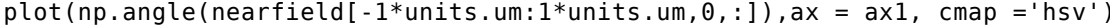
;

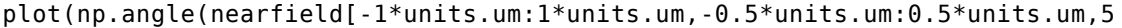
*units.um+wg. l_waveguide]), ax =ax2, cmap ='hsv') ;
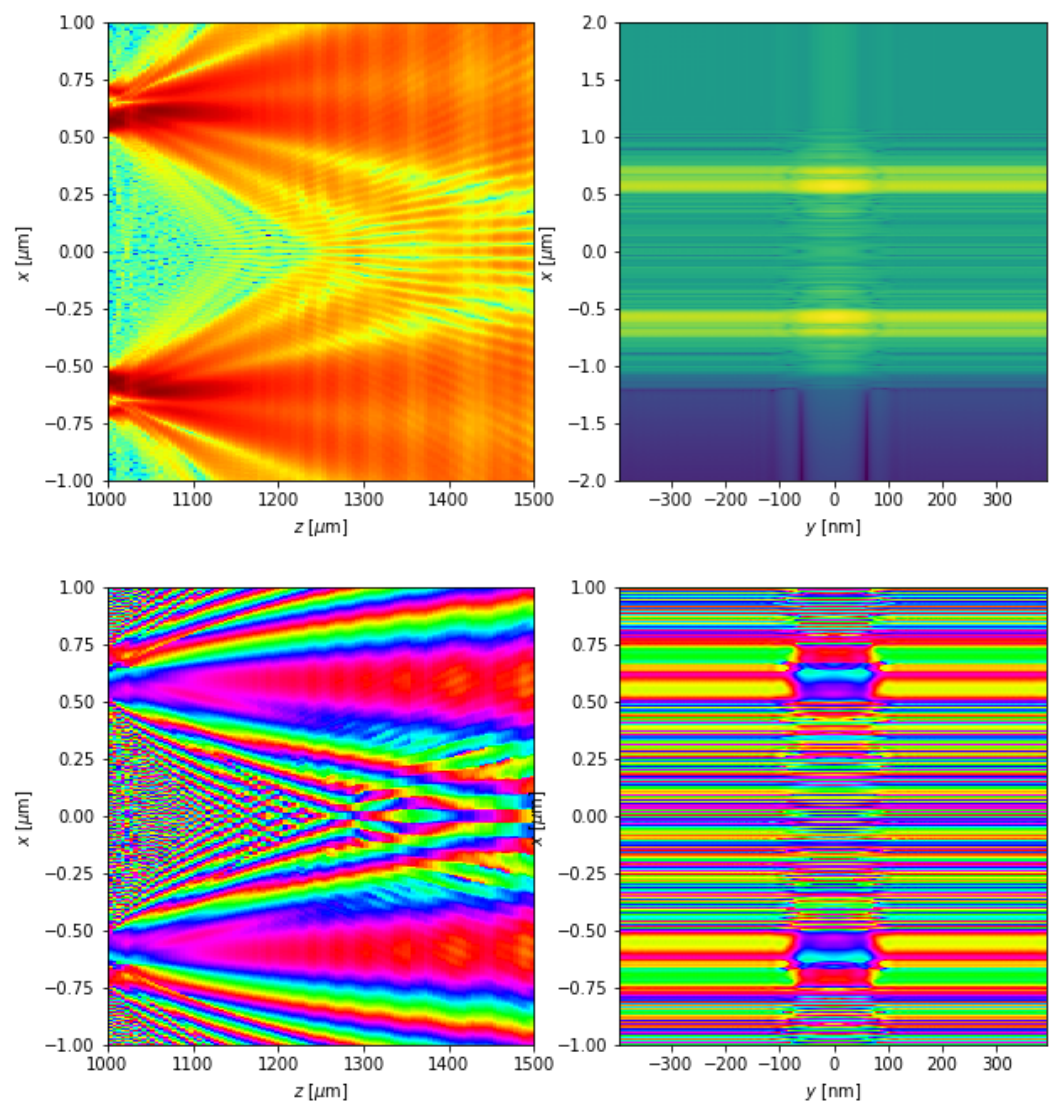


\title{
Delay-line
}

\author{
In [4]: \#Parameter for Variation \\ wg. create_symbol ("l_waveguide",pc.Symbol); \\ l waveguide = pc.parameter ("l waveguide", 1000) \\ wg. l_waveguide $=$ l_waveguide*units.um; \\ \# Scaling-Length of curved part \\ wg.create symbol ("d dl",pc.Symbol); \\ $\mathrm{d} \_\mathrm{dl}=\mathrm{pc} \cdot$ parameter $\left(" \mathrm{~d} \_\mathrm{dl} ", 1\right)$; \\ wg.d $d l=d$ dl*units.mm; \\ \# Stretching variable \\ wg.create symbol ("stretch",pc. Symbol); \\ stretch = pc. parameter ("stretch", 10);
}

Define the index as refraction as a waveguide in vacuum.

In [7]: straight $=p c \cdot$ And $\left(z>=w g \cdot d \_d l+w g \cdot\right.$ Prefocus $,(\operatorname{abs}(x)<=$ wg.r_waveguide $),($ abs $(y)<$ $=w g \cdot r$ waveguide) )

curved $=\left(\operatorname{abs}\left(x^{*}\right.\right.$ stretch $-p c \cdot \cos \left(z /\left(w g \cdot d \_d l\right) *\right.$ math.pi*2) $)<=2 *$ wg.r_waveguide $)$

inside_waveguide $=$ pc.0r(straight, pc. And (curved, abs $(y)<=w g . r$ waveguide, $z<$ $=w g . d$ d l $+w g$. Prefocus ) , $z>=w g$. l waveguide+wg.Prefocus, $z<=w g$. Prefocus)

$\mathrm{s} \cdot \mathrm{n}=\mathrm{pc} \cdot$ piecewise $((\mathrm{n}$ Va, inside waveguide $),(\mathrm{n}$ Si, True $))$

Plot of the index of refraction for review.

In [8]: $n=s . n$.function;

fig, $(a \times 1, a \times 2)=$ plt. $\operatorname{subplots}(1,2$, figsize $=(10,4))$;

plot $(n(s . x, 0, s . z)$, settings, $a x=a x 1) ; p \operatorname{lot}(n(s . x, s \cdot y, 1$ *units.mm), settings, $\mathrm{ax}=\mathrm{ax} 2$ );
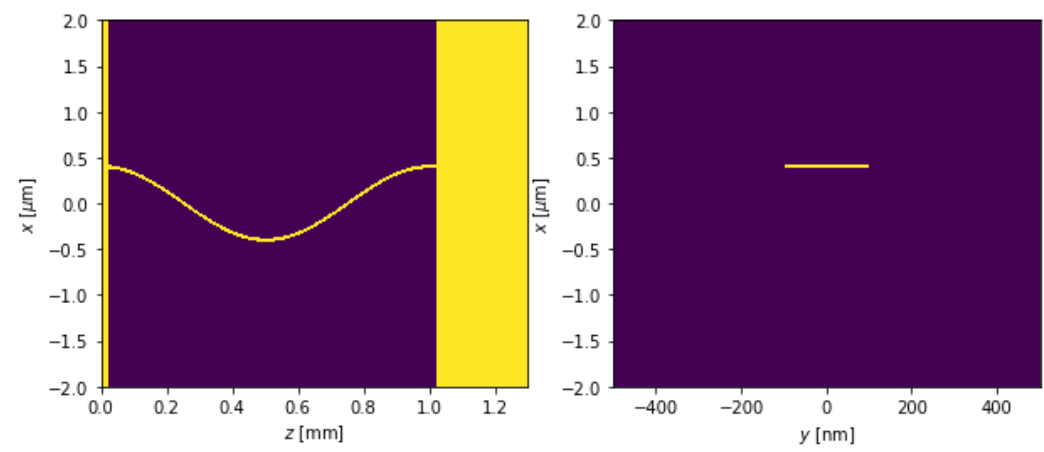
In [14]: propagator $=$ propagators.FiniteDifferences2D (settings)

$A=\operatorname{range}(\theta) ; B=\operatorname{range}(\theta) ; S=\operatorname{range}(\theta)$

steps $=2$

for $i$ in range $(0$, steps $)$ :

stretch.set value $(5+4 * i)$

print "Strētch-Factor \%s" \% stretch.get_value()

field 2D=propagator. run slice()[:]

Variable= "\%s" \%( stretch. get value())

plot $($ field_2D,figsize $=(10,3)$, cmap=fire_colormap ()$)$;

\section{\# Efficiency}

In $=(n p . \operatorname{sum}(n p$. abs (field 2D [ $r$ waveguide $: r$ waveguide,Prefocus $]) * * 2$, axis= S.x)) ;

Out $=$ (np.sum (np.abs (field 2D[0*units.um: $1 *$ units.um, (wg.l waveguide+wg. $\mathrm{Pr}$ efocus)] $) * * 2$, axis=s.x))
A. append (In)
B. append (Out)
S. append (Out/ (In)*100)

print $S$

Stretch-Factor 5

propagating:

$1299 / 1299[0.9 \mathrm{~s}<0(0) \mathrm{s}]]$

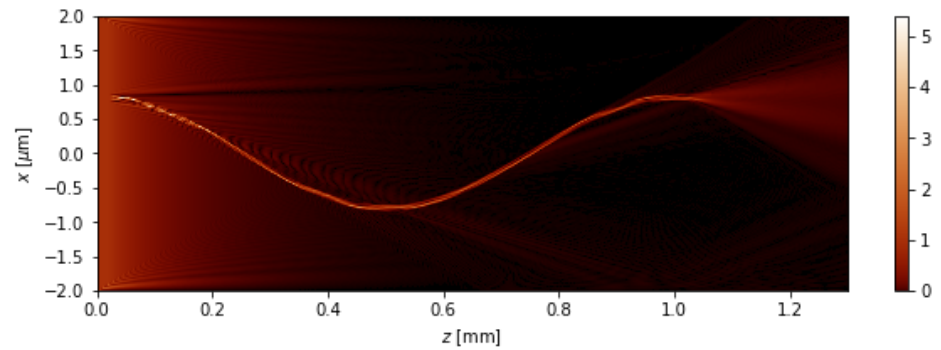

Stretch-Factor 9

propagating:

$1299 / 1299[0.9 \mathrm{~s}<0(0) \mathrm{s}]]$

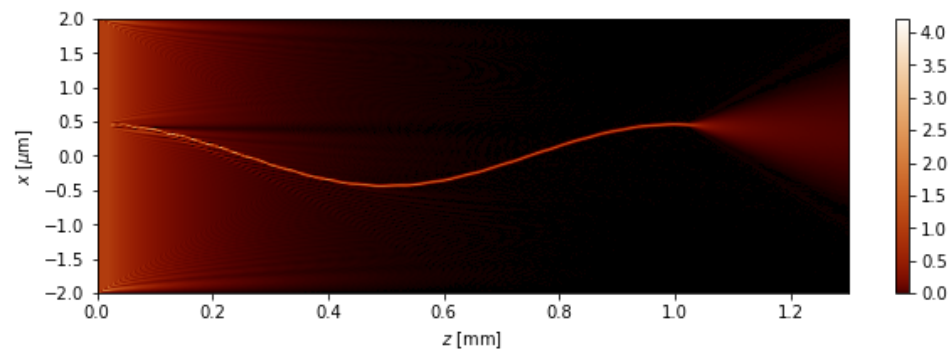

[24.188468149690234, 16.806525113135869] 


\section{Curved channels}

In [3]: wg.create_symbol("R_kruemmung",pc.Symbol);

R_kruemmung = pc.parameter("R_kruemmung",350)

$\mathrm{wg} \cdot \mathrm{R} \_$kruemmung $=\mathrm{R} \_$kruemmung*üits.mm;

wg. create symbol ("l waveguide",pc.Symbol);

l_waveguide = pc.parameter ("l_waveguide",2000)

wg. l_waveguide = l_waveguide*units.um;

Define the index as refraction as a waveguide in vacuum.

In [6]: exprPlus $=$ (wg.R_kruemmung+wg.r_waveguide)

exprMinus $=(\mathrm{wg} \cdot \overline{\mathrm{R}} \mathrm{kruemmung}-\mathrm{wg} \cdot \overline{\mathrm{r}}$ waveguide $)$

expr1 = exprPlus**2 >= ( $z * * 2+(\bar{x}-$ wg.R_kruemmung $) * * 2)$

expr2 $=(\mathrm{z} * * 2+(\mathrm{x}-\mathrm{wg} \cdot \mathrm{R}$ kruemmung $) * * 2)-=$ exprMinus $* * 2$

inside waveguide $=p c \cdot 0 r(p c$. And $(\operatorname{expr} 1, \exp r 2$, abs $(y)<=w g \cdot r$ waveguide $), z>=w g$

.l_waveguide+wg. Prefocus, $z<=w g$. Prefocus)

$\mathrm{s} \cdot \overline{\mathrm{n}}=$ pc.piecewise ( $\left(n \_\right.$Va, inside_waveguide $),($n_Si,True $\left.)\right)$

Plot of the index of refraction for review.

In [7]: $n=s . n . f u n c t i o n ; f i g,(a x 1, a x 2)=\operatorname{plt} . \operatorname{subplots}(1,2, f i g s i z e=(10,4))$; $p \operatorname{lot}(n(s . x, 0, s . z)$, settings, $a x=a x 1) ; p \operatorname{plot}(n(s \cdot x, s \cdot y, 1$ *units.mm), settings, $\mathrm{ax}=\mathrm{ax} 2)$;
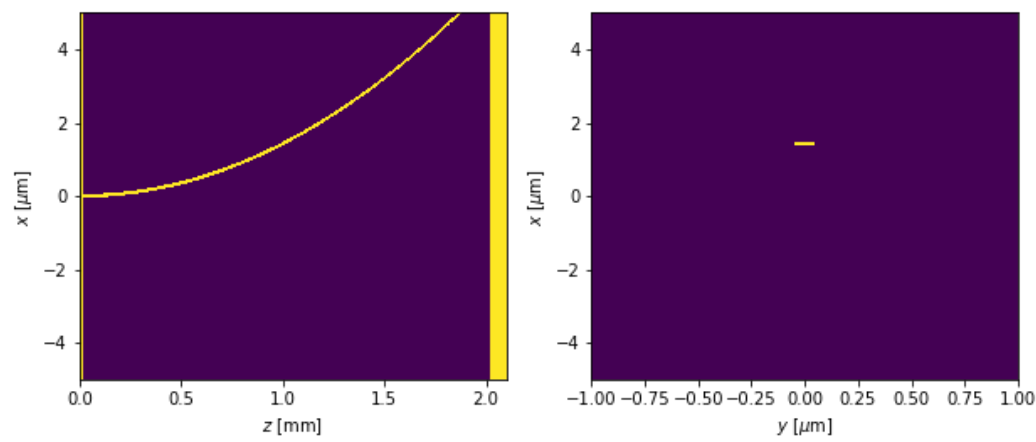


\section{1+1D simulation}
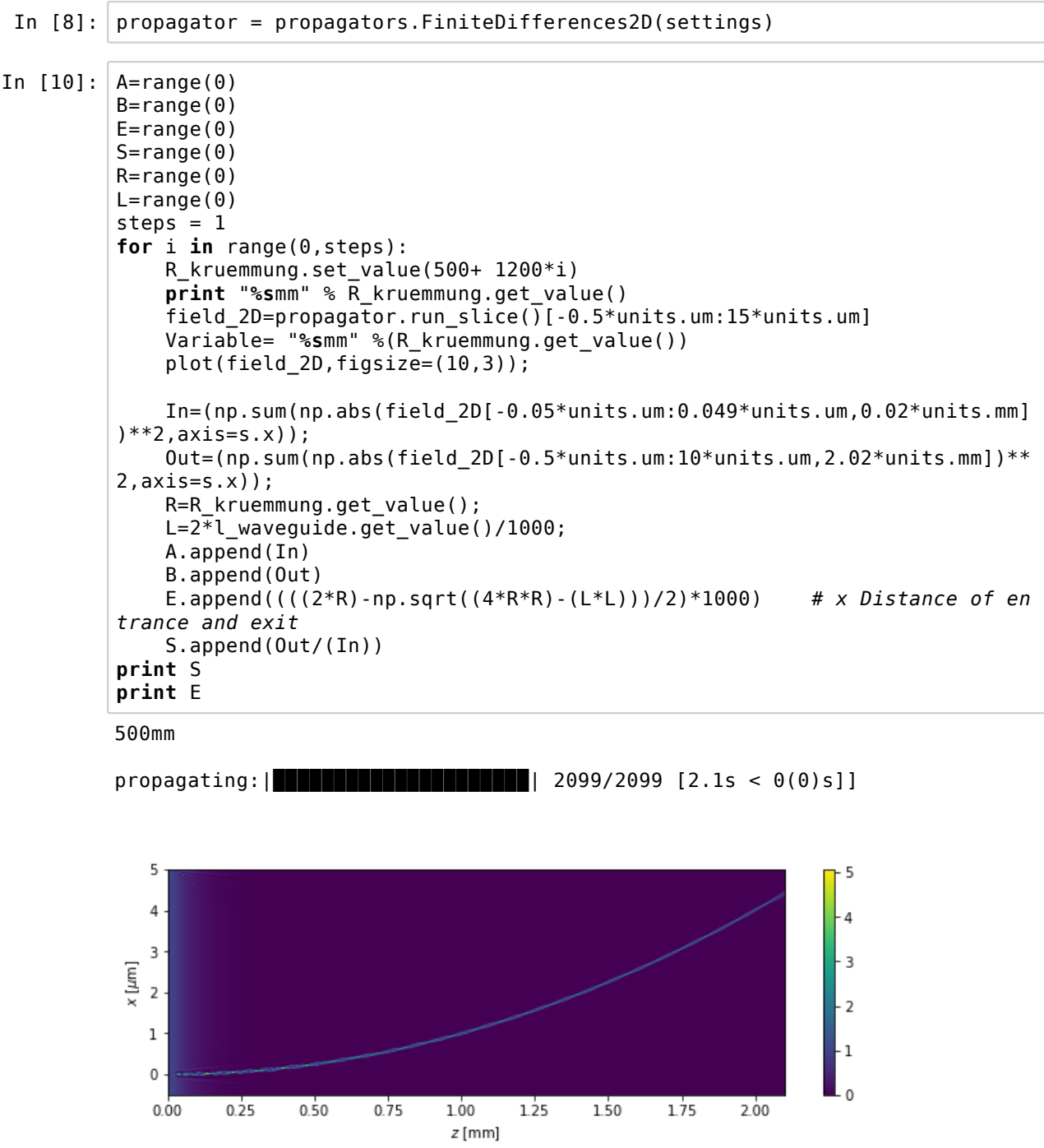

[0.85379401672756861]

[4.0000160001341101] 


\section{Advanced curvatures}

In [4]: \#Parameter for Variation

wg.create_symbol("R_curv",pc.Symbol);

R_curv = p $\bar{c} \cdot$ paramete $\bar{r}$ ("R_curv", 200000)

wg.R_curv= R_curv*units.um;

wg.create_symbol("n_grad",pc.Symbol);

n grad $=p \bar{c}$. paramete $\bar{r}(" n$ grad", $9.4 * 10 * *-5)$

$w \bar{g} \cdot \mathrm{n} \_\mathrm{grad}=w g$. R_curv**-1 ;

Define the index as refraction as a waveguide in vacuum.

In [7]: inside condition square $=(\operatorname{abs}(\mathrm{x}) * * 2<=$ wg. $\mathrm{r}$ waveguide**2) \& (abs $(\mathrm{y}) * * 2<=$ $100 *$ units. nm**2)

inside_waveguide = pc.0r(pc.And(inside_condition_square), z>=wg.l_waveguide+ wg.Prefocus, $z<=w g$. Prefocus)

s.n = pc.piecewise ( $\left(n \_V a-w g \cdot n \_g r a d * x\right.$, inside_waveguide $\left.),\left(n \_S i, T r u e\right)\right)$

Plot of the index of refraction for review.

In [8]: $n=s . n . f u n c t i o n$

fig, $(a x 1, a x 2)=p l t$. $\operatorname{subplots}(1,2$, figsize $=(10,5))$;

plot $(n(s . x, 0, s . z)$, settings, $a x=a x 1) ; \operatorname{plot}\left(n\left(s . x, s . y, 0.5^{*}\right.\right.$ units.mm), settin gs, $a x=a x 2)$;
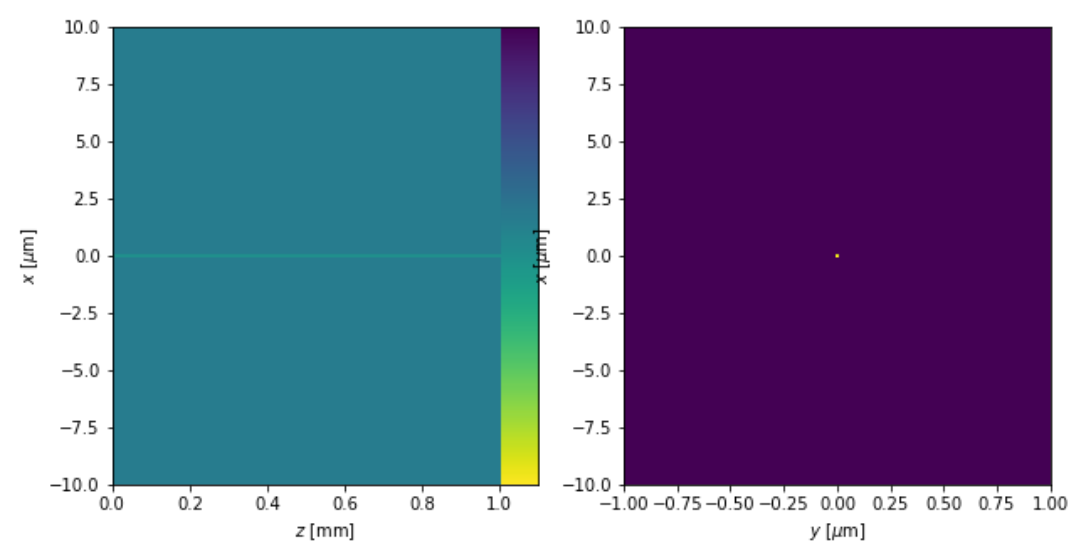

\section{Simulating with gradient}

In [9]: propagator $=$ propagators.FiniteDifferences2D (settings) 
In $[10]: A=\operatorname{range}(0) ; B=\operatorname{range}(0) ; S=\operatorname{range}(0)$

steps $=2$

for $i$ in range $(\theta$, steps):

R_curv.set_value((50000 - 46500*i))

print "\%sum" \% R curv.get_value()

Variable $=$ "\%sum" \%(R_curv.get_value ( ) )

field 2D=propagator.run slice() [: ]

In $=(n \bar{p} . \operatorname{sum}(n p$. abs (field_2D [ - 0.05*units. um: $0.05 *$ units. um, $0.02 *$ units.mm $])$

$* * 2$, axis $=s . x))$;

out $=(\mathrm{np} . \operatorname{sum}(\mathrm{np}$. abs (field 2D [ - $0.05 *$ units.um: $0.05 *$ units.um, $1.02 *$ units.mm $]$ )**2, axis $=\mathrm{s} . \mathrm{x}))$;
A. append (In);
B. append (Out) :
S. append (0ut/ (In)*100) ;
plot (field_2D[-0.5*units.um:0.5*units.um],figsize $=(15,5))$; print $\mathrm{S}$

50000 um

propagating:

$1099 / 1099[2.1 \mathrm{~s}<0(0) \mathrm{s}]]$

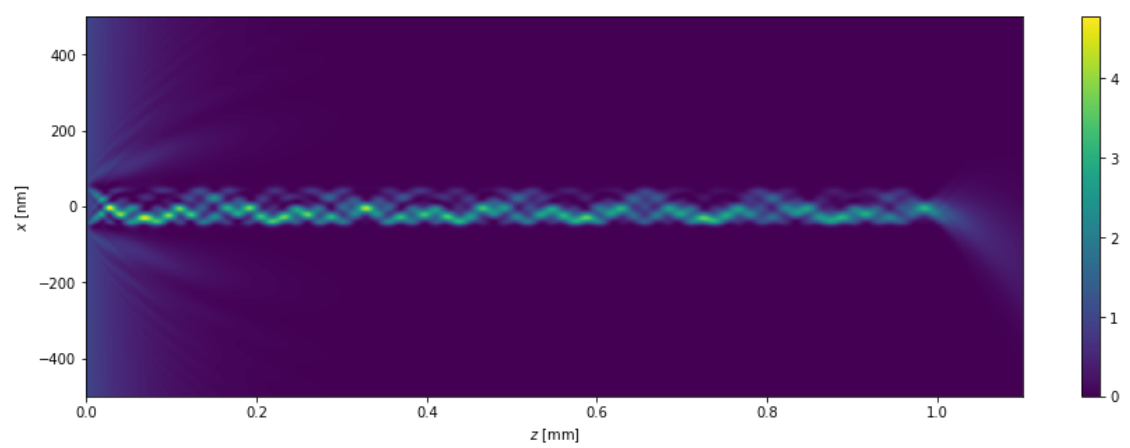

3500 um

propagating:

$1099 / 1099[1.9 \mathrm{~s}<0(0) \mathrm{s}]]$

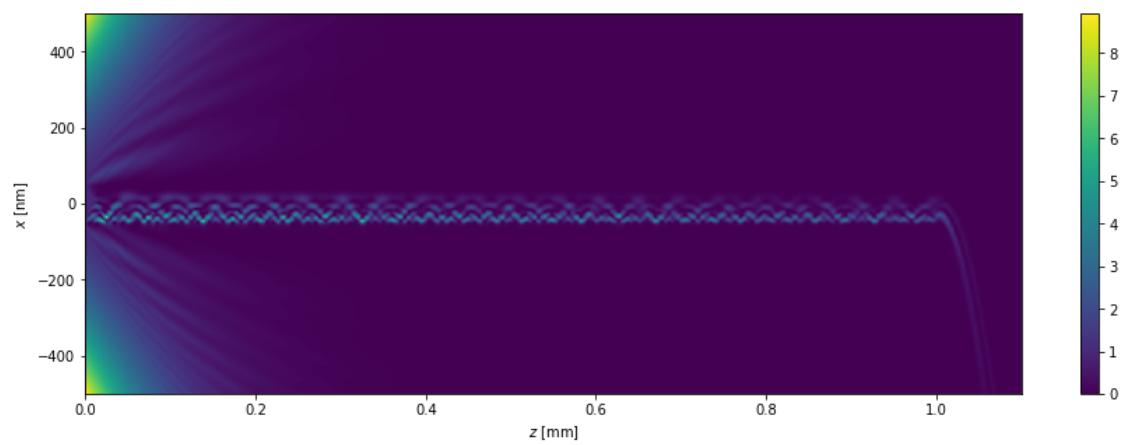

[55.306684538631501，11.844745917566923] 


\section{Simulating with rotating coordinatesystem}

In [11]: wg.R_curv= $0^{*}$ units.um; wg.n_grad=0;

In [12]: def create_curvature_initializer(R):

def curvature_initializer(settings):

$\mathrm{s}=$ settings.symbols

alpha $=$ settings.get_as $(-p c \cdot \operatorname{atan}(s \cdot d z / R), f l o a t)$

update field $=$ expression to $\operatorname{array}\left(p c \cdot \exp \left(1 j * s \cdot k^{*} s \cdot x^{*} p c \cdot \sin (a l p h a)\right)\right.$

, settings). data

def curvature updater(propagator):

field = propagator._get_field ()

field $*=$ update fie $\bar{d}$

settings.updaters ['curvature'] = curvature_updater

return curvature initializer

settings.initializers['curvature'] = create_curvature_initializer(50*units. $\mathrm{mm}$ )

In [13]: propagator = propagators.FiniteDifferences2D (settings)

field_2D=propagator.run_slice() [:] ;

plot (field_2D[-0.5*units.um: $0.5^{*}$ units.um], figsize $\left.=(15,5)\right)$;

propagating:

$1099 / 1099[2.2 \mathrm{~s}<0(0) \mathrm{s}]]$

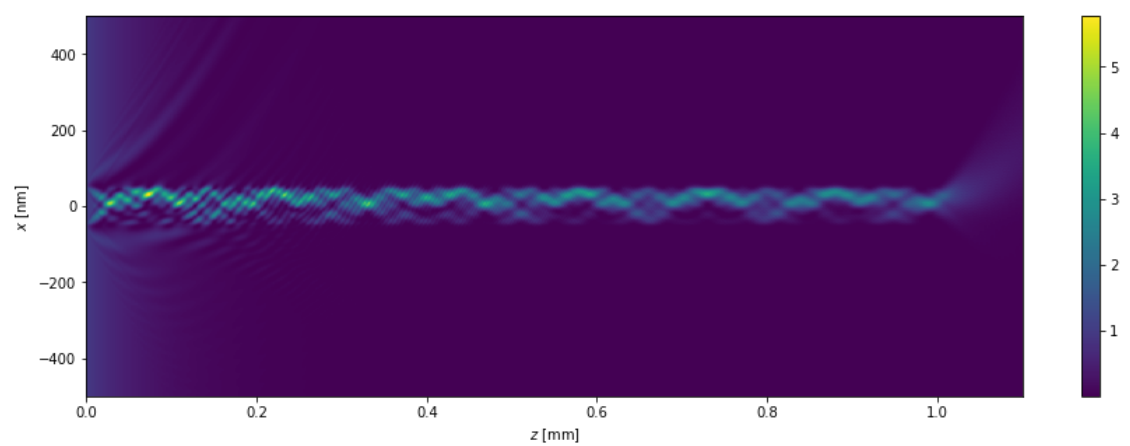




\section{Bibliography}

[1] V.E. Cosslett and W. C. Nixon, X-ray Microscopy. Cambridge. Eng. University press, 1960.

[2] E. Abbe, "Beiträge zur Theorie des Mikroskops und der mikroskopischen Wahrnehmung," Archiv für mikroskopische Anatomie, vol. 9, no. 1, pp. 413-418, 1873.

[3] W. Röntgen, "Über eine neue Art von Strahlen," Sitzungsberichte der physikal.medizin. Gesellschaft, vol. 137, pp. 132-141, 1895.

[4] A. Beer, "Bestimmung der Absorption des rothen Lichts in farbigen Flüssigkeiten," Annalen der Physik und Chemie, vol. 86, no. 2, p. 1852, 1852.

[5] S. Lagomarsino, A. Cedola, P. Cloetens, S. Di Fonzo, W. Jark, G. Soullie, and C. Riekel, "Phase contrast hard x-ray microscopy with submicron resolution," Appl. Phys. Lett., vol. 71, no. 18, pp. 2557-2559, 1997.

[6] A. Cedola and S. Lagomarsino, "From surface x-ray standing waves to waveguides: Principles and applications," Synchrotron Radiation News, vol. 17, no. 3, pp. 3036, 2004.

[7] P. Cloetens, W. Ludwig, J. Baruchel, J.-P. Guigay, P. Pernot-Rejmánková, M. SaloméPateyron, M. Schlenker, J.-Y. Buffière, E. Maire, and G. Peix, "Hard X-ray phase imaging using simple propagation of a coherent synchrotron radiation beam," Journal of Physics D: Applied Physics, vol. 32, no. 10A, p. A145, 1999.

[8] M. Krenkel, M. Bartels, and T. Salditt, "Transport of intensity phase reconstruction to solve the twin image problem in holographic x-ray imaging," Optics express, vol. 21, no. 2, pp. 2220-2235, 2013.

[9] D. Gabor, “A new microscopic principle,” Nature, vol. 161, pp. 777-778, 1948.

[10] L. D. Caro, C. Giannini, D. Pelliccia, C. Mocuta, T. H. Metzger, A. Guagliardi, A. Cedola, I. Burkeeva, and S. Lagomarsino, "In-line holography and coherent diffractive imaging with x-ray waveguides," Physical Review B (Condensed Matter and Materials Physics), vol. 77, no. 8, p. 081408, 2008. 
[11] K. Giewekemeyer, H. Neubauer, S. Kalbfleisch, S. P. Krüger, and T. Salditt, "Holographic and diffractive x-ray imaging using waveguides as quasi-point sources," New J. Phys., vol. 12, p. 035008, 2010.

[12] S. Kalbfleisch, H. Neubauer, S. P. Krüger, M. Bartels, M. Osterhoff, D. D. Mai, K. Giewekemeyer, B. Hartmann, M. Sprung, and T. Salditt, "The Göttingen holography endstation of beamline P10 at PETRA III/DESY," AIP Conf. Proc., vol. 1365, pp. 96-99, 2011.

[13] C. Fuhse, C. Ollinger, and T. Salditt, "Waveguide-based off-axis holography with hard x-rays," Phys. Rev. Lett., vol. 97, p. 254801, 2006.

[14] J. R. Fienup, “Phase retrieval algorithms: A comparison,” Appl. Opt., vol. 21, no. 15, pp. 2758-2769, 1982.

[15] A. Pein, S. Loock, G. Plonka, and T. Salditt, "Using sparsity information for iterative phase retrieval in x-ray propagation imaging," Optics express, vol. 24, no. 8, pp. 8332-8343, 2016.

[16] P. Kirkpatrick and A. V. Baez, "Formation of optical images by x-rays," J. Opt. Soc. Am., vol. 38, no. 9, pp. 766-773, 1948.

[17] L.-J. Lu, "Prefocusing optics for soft-X-ray synchrotron-radiation monochromators,” Appl. Opt., vol. 35, no. 19, pp. 3627-3634, 1996.

[18] Z. Zhong, C. Kao, D. Siddons, and J. Hastings, "Sagittal focusing of high-energy synchrotron x-rays with asymmetric Laue crystals. I. theoretical considerations," Journal of Applied Crystallography, vol. 34, no. 4, pp. 504-509, 2001.

[19] D. H. Bilderback, S. A. Hoffman, D. J. Thiel, et al., "Nanometer spatial resolution achieved in hard x-ray imaging and Laue diffraction experiments," Science, vol. 263, no. 5144, pp. 201-203, 1994.

[20] A. Snigirev, V. Kohn, I. Snigireva, B. Lengeler, et al., "A compound refractive lens for focusing high-energy X-rays," Nature, vol. 384, no. 6604, pp. 49-51, 1996.

[21] B. Lengeler, C. Schroer, J. Tümmler, B. Benner, M. Richwin, A. Snigirev, I. Snigireva, and M. Drakopoulos, "Imaging by parabolic refractive lenses in the hard x-ray range,” Journal of Synchrotron Radiation, vol. 6, no. 6, pp. 1153-1167, 1999.

[22] C. David, J. Bruder, T. Rohbeck, C. Grünzweig, C. Kottler, A. Diaz, O. Bunk, and F. Pfeiffer, "Fabrication of diffraction gratings for hard x-ray phase contrast imaging,” Microelectronic Engineering, vol. 84, no. 5, pp. 1172-1177, 2007.

[23] B. Niemann, D. Rudolph, and G. Schmahl, "Soft x-ray imaging zone plates with large zone numbers for microscopic and spectroscopic applications," Opt. Commun., vol. 12, pp. 160-163, 1974. 
[24] K. Saitoh, K. Inagawa, K. Kohra, C. Hayashi, A. Iida, and N. Kato, "Fabrication and characterization of multilayer zone plate for hard x-rays," Japanese J. Appl. Phys., vol. 27, no. 11A, p. L2131, 1988.

[25] J. Maser, G. B. Stephenson, S. Vogt, W. Yun, A. Macrander, H. C. Kang, C. Liu, and R. Conley, "Multilayer Laue lenses as high-resolution x-ray optics," in Optical Science and Technology, the SPIE 49th Annual Meeting, pp. 185-194, International Society for Optics and Photonics, 2004.

[26] H. Yan, H. C. Kang, R. Conley, C. Liu, A. T. Macrander, G. B. Stephenson, J. Maser, et al., "Multilayer Laue lens: A path toward one nanometer X-ray focusing," X-ray Optics and Instrumentation, vol. 2010, 2010.

[27] E. Spiller and A. Segmüller, "Propagation of x-rays in waveguides," Appl. Phys. Lett., vol. 24, no. 2, pp. 60-61, 1974.

[28] Y. P. Feng, S. K. Sinha, H. W. Deckman, J. B. Hastings, and D. P. Siddons, "X-ray flux enhancement in thin-film waveguides using resonant beam couplers," Phys. Rev. Lett., vol. 71, no. 4, pp. 537-540, 1993.

[29] C. G. Schroer, O. Kurapova, J. Patommel, P. Boye, J. Feldkamp, B. Lengeler, M. Burghammer, C. Riekel, L. Vincze, A. V. D. Hart, and M. Kuchler, "Hard X-ray nanoprobe based on refractive x-ray lenses,” Appl. Phys. Lett., vol. 87, p. 124103, 2005.

[30] H. Yan, V. Rose, D. Shu, E. Lima, H. C. Kang, R. Conley, C. Liu, N. Jahedi, A. T. Macrander, G. B. Stephenson, et al., "Two dimensional hard x-ray nanofocusing with crossed multilayer laue lenses," Optics express, vol. 19, no. 16, pp. 1506915076, 2011.

[31] Y. Suzuki, A. Takeuchi, H. Takano, and H. Takenaka, "Performance test of fresnel zone plate with $50 \mathrm{~nm}$ outermost zone width in hard x-ray region," Japanese journal of applied physics, vol. 44, no. 4R, p. 1994, 2005.

[32] F. Döring, A. Robisch, C. Eberl, M. Osterhoff, A. Ruhlandt, T. Liese, F. Schlenkrich, S. Hoffmann, M. Bartels, T. Salditt, and H. Krebs, "Sub-5 nm hard x-ray point focusing by a combined Kirkpatrick-Baez mirror and multilayer zone plate," Opt. Express, vol. 21, no. 16, pp. 19311-19323, 2013.

[33] A. Jarre, C. Fuhse, C. Ollinger, J. Seeger, R. Tucoulou, and T. Salditt, "Twodimensional hard x-ray beam compression by combined focusing and waveguide optics,” Phys. Rev. Lett., vol. 94, no. 7, p. 074801, 2005.

[34] S. P. Krüger, H. Neubauer, M. Bartels, S. Kalbfleisch, K. Giewekemeyer, P. J. Wilbrandt, M. Sprung, and T. Salditt, "Sub-10 nm beam confinement by x-ray waveguides: Design, fabrication and characterization of optical properties," $J$. Synchrotron Radiat., vol. 19, pp. 227-236, 2012. 
[35] M. Zwanenburg, J. Peters, J. Bongaerts, S. de Vries, D. Abernathy, and J. van der Veen, "Coherent propagation of X-rays in a planar waveguide with a tunable air gap,” Phys. Rev. Lett., vol. 82, no. 8, pp. 1696-1699, 1999.

[36] J. Bongaerts, C. David, M. Drakopoulos, M. J. Zwanenburg, G. Wegdam, T. Lackner, H. Keymeulen, and J. van der Veen, "Propagation of a partially coherent focused x-ray beam within a planar x-ray waveguide," J. Synchrotron Rad., vol. 9, no. 6, pp. 383-393, 2002.

[37] T. Salditt, S. Krüger, C. Fuhse, and C. Bähtz, "High-transmission planar x-ray waveguides,” Physical review letters, vol. 100, no. 18, p. 184801, 2008.

[38] F. Pfeiffer, T. Salditt, P. Høghøj, I. Anderson, and N. Schell, "X-ray waveguides with multiple guiding layers,” Physical Review B, vol. 62, no. 24, p. 16939, 2000.

[39] I. Prudnikov, "X-ray guided modes produced by bragg reflections of multilayers: An analytical treatment," Journal of applied crystallography, vol. 38, no. 4, pp. 595602, 2005.

[40] K. Okamoto, T. Noma, A. Komoto, W. Kubo, M. Takahashi, A. Iida, and H. Miyata, "X-ray waveguide mode in resonance with a periodic structure," Physical review letters, vol. 109, no. 23, p. 233907, 2012.

[41] S. Lagomarsino, W. Jark, S. Di Fonzo, A. Cedola, B. Mueller, P. Engström, and C. Riekel, "Submicrometer x-ray beam production by a thin film waveguide," $J$. Appl. Phys., vol. 79, no. 8, pp. 4471-4473, 1996.

[42] F. Pfeiffer, C. David, M. Burghammer, C. Riekel, and T. Salditt, “Two-dimensional X-ray waveguides and point sources,” Science, vol. 297, no. 5579, pp. 230-234, 2002.

[43] A. Kohlstedt, S. Kalbfleisch, T. Salditt, M. Reiche, U. Gösele, E. Lima, and P. Willmott, "Two-dimensional x-ray waveguides: Fabrication by wafer-bonding process and characterization,” Appl. Phys. A, vol. 91, pp. 6-12, 2008.

[44] T. Beetz and C. Jacobsen, "Soft x-ray radiation-damage studies in pmma using a cryo-stxm,” Journal of Synchrotron Radiation, vol. 10, no. 3, pp. 280-283, 2003.

[45] C. Fuhse, X-ray waveguides and waveguide-based lensless imaging. PhD thesis, University of Göttingen, 2006.

[46] H. Neubauer, Verfahren zur Herstellung neuartiger Röntgenwellenleiter. PhD thesis, University of Göttingen, 2012.

[47] Y. Feng, S. Sinha, E. E. Fullerton, G. Grübel, D. Abernathy, D. Siddons, and J. Hastings, "X-ray Fraunhofer diffraction patterns from a thin-film waveguide," Appl. Phys. Lett., vol. 67, no. 24, pp. 3647-3649, 1995. 
[48] A. Cedola, S. Di Fonzo, W. Jark, S. Lagomarsino, and G. Soullie, "Sub-micrometre coherent beams from x-ray waveguides: Principles and applications," Journal of Physics D: Applied Physics, vol. 32, no. 10A, p. A179, 1999.

[49] S. Hoffmann-Urlaub, P. Höhne, M. Kanbach, and T. Salditt, "Advances in x-ray waveguide fabrication," Microelectronic Engineering, vol. 164, pp. 135-138, 2016.

[50] C. Bergemann, H. Keymeulen, and J. F. van der Veen, "Focusing x-ray beams to nanometer dimensions,” Phys. Rev. Lett., vol. 91, no. 20, p. 204801, 2003.

[51] S. Kalbfleisch, A dedicated endstation for waveguide-based $x$-ray imaging. $\mathrm{PhD}$ thesis, Universität Göttingen, 2012.

[52] S. Hoffmann-Urlaub and T. Salditt, "Miniaturized beamsplitters realized by x-ray waveguides,” Acta Crystallographica Section A, vol. 72, pp. 515-522, 2016.

[53] L. Melchior, "Pypropagate." https://github.com/TheLartians/ PyPropagate, 2016.

[54] D. Marcuse, "Theory of dielectric optical waveguides," Academic Press, New York, 1974.

[55] A. Jarre, Hard $x$-ray waveguide optics. PhD thesis, University of Göttingen, 2005.

[56] B. L. Henke, E. M. Gullikson, and J. C. Davis, "X-ray interactions: Photoabsorption, scattering, transmission, and reflection at $\mathrm{E}=50-30,000 \mathrm{eV}, \mathrm{Z}=1-92$," At. Data Nucl. Data Tables, vol. 54, no. 2, pp. 181-342, 1993.

[57] L. Ognev, "Decrease in the incoherent near-edge x-ray scattering in narrow waveguides,” Technical Physics Letters, vol. 36, no. 2, pp. 133-135, 2010.

[58] C. Fuhse and T. Salditt, "Propagation of x-rays in ultra-narrow slits," Opt. Commun., vol. 265, no. 1, pp. 140-146, 2006.

[59] J. Yeom, Y. Wu, J. C. Selby, and M. A. Shannon, "Maximum achievable aspect ratio in deep reactive ion etching of silicon due to aspect ratio dependent transport and the microloading effect," Journal of Vacuum Science \& Technology B, vol. 23, no. 6, pp. 2319-2329, 2005.

[60] C.-K. Chung, "Geometrical pattern effect on silicon deep etching by an inductively coupled plasma system," Journal of Micromechanics and Microengineering, vol. 14, no. 4, p. 656, 2004.

[61] R. A. Gottscho, C. W. Jurgensen, and D. J. Vitkavage, "Microscopic uniformity in plasma etching," Journal of Vacuum Science \& Technology B, vol. 10, no. 5, pp. 2133-2147, 1992.

[62] J. Coburn and H. F. Winters, "Conductance considerations in the reactive ion etching of high aspect ratio features," Applied Physics Letters, vol. 55, no. 26, pp. 27302732, 1989. 
[63] H. Jansen, M. de Boer, R. Wiegerink, N. Tas, E. Smulders, C. Neagu, and M. Elwenspoek, "RIE-lag in high aspect ratio trench etching of silicon," Microelectronic Engineering, vol. 35, no. 1, pp. 45-50, 1997.

[64] E. Blumenröther, "Der Trockenätzprozess bei der Herstellung von Röntgenwellenleitern: Optimierung der Ätzparameter bzgl. Strukturbreite und Resistdicke," Bachelor's thesis, Georg-August University of Göttingen, Germany, 2010.

[65] M. V. Bazylenko, M. Gross, and M. Faith, "Effect of reactive ion etching-generated sidewall roughness on propagation loss of buried-channel silica waveguides," $A p$ plied Physics Letters, vol. 69, no. 15, pp. 2178-2180, 1996.

[66] M. Osterhoff and T. Salditt, "Real structure effects in x-ray waveguide optics: The influence of interfacial roughness and refractive index profile on the near-field and far-field distribution," Opt. Commun., vol. 282, no. 16, pp. 3250-3256, 2009.

[67] H. F. Talbot, "LXXVI. Facts relating to optical science. No. IV,” The London and Edinburgh Philosophical Magazine and Journal of Science, vol. 9, no. 56, pp. 401407, 1836.

[68] L. Ognev, "Extinction depth in a smooth x-ray waveguide," Technical Physics, vol. 60, no. 10, pp. 1525-1528, 2015.

[69] I. Bukreeva, A. Cedola, A. Sorrentino, D. Pelliccia, V. Asadchikov, and S. Lagomarsino, "Resonance modes filtering in structured x-ray waveguides," Opt. Lett., vol. 36, no. 14, pp. 2602-2604, 2011.

[70] R. Edgar, “The Fresnel diffraction images of periodic structures," Journal of Modern Optics, vol. 16, no. 3, pp. 281-287, 1969.

[71] T. Salditt, S. Hoffmann, M. Vassholz, J. Haber, M. Osterhoff, and J. Hilhorst, "Xray optics on a chip: Guiding X-rays in curved channels," Phys. Rev. Lett., vol. 115, p. 203902, 2015.

[72] M. Heiblum and J. H. Harris, "Analysis of curved optical waveguides by conformal transformation," IEEE Journal of Quantum Electronics, vol. 11, pp. 75-83, 1975.

[73] C. Liu and J. A. Golovchenko, "Surface trapped x-rays: Whispering-gallery modes at $\lambda=0.7 \AA$," Phys Rev Lett, vol. 79, no. 5, p. 788, 1997.

[74] D. Pelliccia, "X-ray photonics: Bending x-rays with nanochannels," Nature Photonics, vol. 10, no. 2, pp. 75-77, 2016.

[75] A. Brünner, "http://www.arndt-bruenner.de/mathe/scripts/kreissehnen.htm," July, 2015.

[76] S. Marchesini, H. He, H. N. Chapman, S. P. Hau-Riege, A. Noy, M. R. Howells, U. Weierstall, and J. C. Spence, "X-ray image reconstruction from a diffraction pattern alone,” Physical Review B, vol. 68, no. 14, p. 140101, 2003. 
[77] Webpage of the Helmholtz Zentrum Dresden Rossendorf. http://www.hzdr. $\mathrm{de} / \mathrm{db} / \mathrm{Cms}$ ?pNid=146

[78] T. D. Milster and N. A. Beaudry, "Coherence and fringe localization." http://www.tau.ac.il/ lab3/OPTICFIBERS/Coherence\%20and\%20Fringe.pdf, 2006.

[79] Webpage of the European Synchrotron Radiation Facility. http://www . esrf.eu/home/UsersAndScience/Expriments/StructMaterials/ID11/ technical-overview.html

[80] G. Vaughan, J. Wright, A. Bytchkov, C. Curfs, C. Gundlach, M. Orlova, L. Erra, H. Gleyzolle, T. Buslaps, A. Götz, et al., "The extension of ID11 for nanoscale and hierarchical characterization," in Risø International Symposium on Materials Science. Proceedings, 2010.

[81] P. Heimann, M. MacDonald, B. Nagler, H. J. Lee, E. Galtier, B. Arnold, and Z. Xing, "Compound refractive lenses as prefocusing optics for x-ray FEL radiation," J Synchrotron Radiat., vol. 23, no. 2, pp. 425-429, 2016.

[82] M. Zwanenburg, J.Bongaerts, J. Peters, D. Riese, and J. van der Veen, "Focusing of coherent x-rays in a tapered planar waveguide," Physica B, vol. 283, no. 13, pp. 285 $-288,2000$.

[83] I. Bukreeva, D. Pelliccia, A. Cedola, F. Scarinci, M. Ilie, C. Giannini, L. De Caro, and S. Lagomarsino, "Analysis of tapered front-coupling x-ray waveguides," J. Synchrotron Radiat., vol. 17, no. 1, pp. 61-68, 2010.

[84] H. Chen, S. Hoffmann, and T. Salditt, "X-ray beam compression by tapered waveguides,” Appl. Phys. Lett., vol. 106, no. 19, p. 194105, 2015.

[85] M. Berry, "Much ado about nothing: Optical dislocation lines (phase singularities, zeros, vortices...)," in Proc. SPIE, vol. 3487, 1998.

[86] K. M. Pavlov, D. M. Paganin, D. J. Vine, J. A. Schmalz, Y. Suzuki, K. Uesugi, A. Takeuchi, N. Yagi, A. Kharchenko, G. Blaj, J. Jakubek, M. Altissimo, and J. N. Clark, "Quantized hard-x-ray phase vortices nucleated by aberrated nanolenses," Phys. Rev. A, vol. 83, p. 013813, 2011.

[87] H. Wolter, "Zur Frage des Lichtweges bei Totalreflexion," Zeitschrift für Naturforschung A, vol. 5, no. 5, pp. 276-283, 1950.

[88] J. E. Curtis, B. A. Koss, and D. G. Grier, "Dynamic holographic optical tweezers," Optics Communications, vol. 207, no. 1, pp. 169-175, 2002.

[89] P. Mao and J. Han, "Fabrication and characterization of $20 \mathrm{~nm}$ planar nanofluidic channels by glass-glass and glass-silicon bonding," Lab on a Chip, vol. 5, no. 8, pp. 837-844, 2005. 
[90] W. Reisner, K. J. Morton, R. Riehn, Y. M. Wang, Z. Yu, M. Rosen, J. C. Sturm, S. Y. Chou, E. Frey, and R. H. Austin, "Statics and dynamics of single DNA molecules confined in nanochannels,” Physical Review Letters, vol. 94, no. 19, p. 196101, 2005.

[91] P. Höhne, “Quartz as a new material for lithographic x-ray waveguides,” Bachelor's thesis, Georg-August University of Göttingen, Germany, 2016.

[92] F. Döring, "Herstellung und charakterisierung von submikrometer germanium wellenleitern und blenden," Master's thesis, Georg-August University of Göttingen, Germany, 2017.

[93] Z. Yu, L. Chen, W. Wu, H. Ge, and S. Y. Chou, "Fabrication of nanoscale gratings with reduced line edge roughness using nanoimprint lithography," Journal of Vacuum Science \& Technology B, vol. 21, no. 5, pp. 2089-2092, 2003. 


\section{Authors contribution}

In this chapter detailed information about the particular contributions of the author in the publications is given. The four peer-reviewed articles reprinted in Chapters 2-5 fulfill the requirements to be considered in a cumulative thesis according to the doctoral degree regulations of the $\mathrm{PhD}$ program ProPhys in the Georg-August University School of Science (GAUSS) Göttingen:

(1) H. Neubauer, S. Hoffmann, M. Kanbach, J. Haber, S. Kalbfleisch, S. Krüger, and T. Salditt High aspect ratio $x$-ray waveguide channels fabricated by e-beam lithography and wafer bonding. Journal of Applied Physics, 115(21):214305 (2014).

(2) H.-Y. Chen, S. Hoffmann and T. Salditt. X-ray beam compression by tapered waveguides Applied Physics Letters, 106(19), 194105 (2015).

(3) S. Hoffmann-Urlaub, P. Höhne, M. Kanbach and T. Salditt. Advances in x-ray waveguide fabrication Microelectronic Engineering, 164, 135-138 (2016).

(4) S. Hoffmann-Urlaub and T. Salditt. Miniaturized beamsplitters realized by $x$-ray waveguides. Acta Crystallographica Section A, 72, 515-522 (2016).

In (1) the fabrication process of waveguides was further improved and samples (II-IV) were both manufactured and characterized at synchrotron sources by the author. Also the infrared light setup was built and the corresponding inspections were carried out. The sample fabrication and measurements in (2) result from the close co-operation of Hsin-Yi Chen and the author. Likewise the author contributed in the analysis of the collected data in the first two publications.

The manuscripts (3) and (4) show the work by the author, with the fluorescence image from the Bachelors thesis of Philipp Höhne as the sole exception.

The results were discussed and interpreted by all authors of the respective publications. The manuscripts of the publications (1) and (2) were written by the first authors, the author and Tim Salditt; (3) and (4) were written by the author and Tim Salditt. Apart from the listed authors, the measurements at the synchrotron sources were supported by collaborators acknowledged within the manuscripts. 



\section{Acknowledgements}

Als erstes möchte ich mich bei meinem Betreuer Prof. Dr. Tim Salditt bedanken, der mir zum Quereinstieg in die Röntgenphysik ein spannendes Thema anbot, wobei er mir Freiraum ließ meine eigenen Ansätze zu verfolgen; aber auch stets beratend und unterstützend zur Seite stand. Seine Fähigkeit die unterschiedlichsten Konzepte miteinander zu verweben, sich für die verschiedensten und noch so unscheinbaren Phänomene zu begeistern und die Bereitschaft sich gedanklich immer wieder auf neue Ansätze einzulassen, haben mich immer motiviert und inspiriert.

Prof. Dr. Hans-Ulrich Krebs möchte ich nicht nur für die Übernahme des Korreferats und das Begleiten meiner Arbeit danken, sondern auch dafür, dass er sich allen Dingen mit einem Quäntchen Humor zuwendet.

Des Weiteren möchte ich der gesamten Prüfungskommission in Gestalt von Dr. A. Egner, Prof. Dr. S. Köster, Prof. Dr. A. Rizzi und Prof. Dr. A. Tilgner dafür danken, dass sie Interesse an meiner Forschung gezeigt und hierein Zeit investiert haben.

Vom reichen Erfahrungsschatz der Vorgänger/innen* E. Blumenröther, C. Fuhse, J. Haber, A. Hillmann, A. Jarre, S. Kalbfleisch, D. Köhne, S.-P. Krüger, H. Neubauer, M. Osterhoff, S. Pankin, F. Pfeiffer und Kai Yun, die sich den Röntgenwellenleitern vor mir gewidmet haben, habe ich sehr profitiert und hoffe, dass nachfolgend viele weitere Menschen Spaß an diesem vielfältigen Thema haben.

Dem technischen Support an den Beamlines durch C. Bähtz, (ROBL, ESRF), J. Hilhorst (ID01, ESRF), C. Krywka (P03, DESY), M. Sprung, (P10, DESY) und J. Wright (ID11, ESRF) und den Großforschungseinrichtungen selbst für ihre finanzielle Unterstützung, möchte ich Danke sagen.

Auch intern wurde auf technischer und infrastruktureller Seite fantastisch gearbeitet, damit die Wellenleiterherstellung funktionierte; ermöglicht haben das Jan Goemann, Bastian Hartmann, Jochen Herbst und Mike Kanbach, dem die kleinen Kanäle besonders am Herzen liegen. Dies trifft auch auf Ferdinand Döring und Philipp Höhne zu, deren Abschlussarbeiten ich betreut habe. Ihre ansteckende Neugier hat mir häufig selbst zu neuen Sichtweisen auf bekannte Thematiken verholfen.

Die Atmosphäre im Institut, die von Interesse, Anteilnahme und Zusammenarbeit geprägt ist, habe ich immer als sehr bereichernd empfunden, was insbesondere auf die bunt besetzten Gesprächsrunden nach dem Mittagessen und die Messzeiten zutrifft. Nicht nur fachlich waren mir die Diskussionen mit Henrike Neubauer, Anna-Lena Robisch und Hsin-Yi Chen eine große Hilfe, sondern auch kulinarisch (ergänzt durch Marius Priebe) und menschlich haben sie mich weitergebildet. 
Auch die Menschen ganz abseits der Forschung haben einen nicht gering zu schätzenden Anteil am Gelingen dieser Arbeit. Für die Zerstreuung und die Hilfe beim Auftanken neuer Kräfte möchte ich der Myers-Runde, dem Rugby-Team und meinen Freunden ohne Gruppenzuordnung ganz herzlich bedanken!

Ohne die Liebe und Unterstützung meiner Familie und meiner Frau Christina, die immer an mich geglaubt und mir den Rücken frei gehalten hat, hätte ich die letzten Jahre nicht so frei verbringen können. Dafür möchte ich euch von ganzem Herzen danken! 